Universidad deValladolid

PROGRAMA DE DOCTORADO EN

INVESTIGACIÓN EN CIENCIAS DE LA SALUD

TESIS DOCTORAL:

\title{
VALIDACIÓN EXTERNA Y ANÁLISIS COMPARATIVO DE ESCALAS PREDICTORAS DEL RIESGO DE COMPLICACIONES TRAS CIRUGÍA DE PÁNCREAS
}

Presentada por Francisco Javier Tejero Pintor para optar al grado de Doctor por la Universidad de Valladolid

Dirigida por:

Baltasar Pérez Saborido

Mario Rodríguez López

David Pacheco Sánchez 

Universidad deValladolid

\section{AUTORIZACIÓN DEL DIRECTOR/A DE TESIS}

Impreso 1T

(Art. 7.2 de la Normativa para la presentación y defensa de la Tesis Doctoral en la UVa)

D. Baltasar Pérez Saborido, con D.N.I. 33389848N Profesor asociado del departamento de Cirugía, Oftalmología, Otorrinolaringología y Fisioterapia de la Facultad de Medicina de la Universidad de Valladolid como Director de la Tesis Doctoral titulada "Validación externa y análisis comparativo de escalas predictoras del riesgo de complicaciones tras cirugía de páncreas" realizada por D. Francisco Javier Tejero Pintor alumno del Programa de Doctorado "Investigación en Ciencias de la Salud", impartido por el departamento de Biología Celular, Histología y Farmacología, autoriza su presentación, considerando que la estructura y ejecución de este trabajo se ajusta a los objetivos propuestos inicialmente en su redacción y por ello puede ser presentada como trabajo original para la obtención del Título de Doctor.

Valladolid 1 de septiembre de 2019

El Director de la Tesis,

Fdo.: Prof. Dr. Baltasar Pérez Saborido

SR/SRA. PRESIDENTE/A DE LA COMISIÓN DE DOCTORADO 

Universidad deValladolid

\section{AUTORIZACIÓN DEL DIRECTOR/A DE TESIS}

Impreso $1 \mathrm{~T}$

(Art. 7.2 de la Normativa para la presentación y defensa de la Tesis Doctoral en la UVa)

D. Mario Rodríguez López, con D.N.I. 12417438Z Profesor asociado del departamento de Cirugía, Oftalmología, Otorrinolaringología y Fisioterapia de la Facultad de Medicina de la Universidad de Valladolid como Director de la Tesis Doctoral titulada "Validación externa y análisis comparativo de escalas predictoras del riesgo de complicaciones tras cirugía de páncreas" realizada por D. Francisco Javier Tejero Pintor alumno del Programa de Doctorado "Investigación en Ciencias de la Salud", impartido por el departamento de Biología Celular, Histología y Farmacología, autoriza su presentación, considerando que la estructura y ejecución de este trabajo se ajusta a los objetivos propuestos inicialmente en su redacción y por ello puede ser presentada como trabajo original para la obtención del Título de Doctor.

Valladolid 1 de septiembre de 2019

El Director de la Tesis,

Fdo.: Prof. Dr. Mario Rodríguez López 

Universidad deValladolid

Impreso 1T

\section{AUTORIZACIÓN DEL DIRECTOR/A DE TESIS}

(Art. 7.2 de la Normativa para la presentación y defensa de la Tesis Doctoral en la UVa)

D. David Pacheco Sánchez, con D.N.I. 07481413I Profesor asociado del departamento de Cirugía, Oftalmología, Otorrinolaringología y Fisioterapia de la Facultad de Medicina de la Universidad de Valladolid como Director de la Tesis Doctoral titulada "Validación externa y análisis comparativo de escalas predictoras del riesgo de complicaciones tras cirugía de páncreas" realizada por D. Francisco Javier Tejero Pintor alumno del Programa de Doctorado "Investigación en Ciencias de la Salud", impartido por el departamento de Biología Celular, Histología y Farmacología, autoriza su presentación, considerando que la estructura y ejecución de este trabajo se ajusta a los objetivos propuestos inicialmente en su redacción y por ello puede ser presentada como trabajo original para la obtención del Título de Doctor.

Valladolid 1 de septiembre de 2019

El Director de la Tesis,

Fdo.: Prof. Dr. David Pacheco Sánchez 



\section{"VALIDACIÓN EXTERNA Y ANÁLISIS \\ COMPARATIVO DE ESCALAS PREDICTORAS \\ DEL RIESGO DE COMPLICACIONES TRAS \\ CIRUGÍA DE PÁNCREAS"}

DOCTORANDO: Francisco Javier Tejero Pintor

DIRECTORES: Prof. Dr. Baltasar Pérez Saborido

Prof. Dr. Mario Rodríguez López

Prof. Dr. David Pacheco Sánchez

CENTRO: $\quad$ Facultad de Medicina,

Universidad de Valladolid, España.

DEPARTAMENTO: Biología Celular, Histología y Farmacología

FINALIZACIÓN: $\quad$ Agosto de 2019. 

"Las habilidades quirúrgicas no pueden ser ganadas mediante la observación, así como las habilidades de tocar el violín no pueden obtenerse escuchando $y$ viendo a un virtuoso tocar este instrumento" Allen Oldfather Whipple 



\section{ÍNDICE}

\section{ÍNDICE.}

I

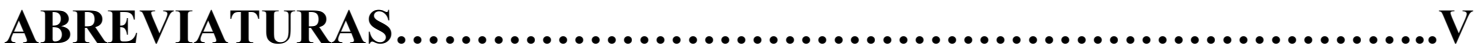

AGRADECIMIENTOS............................................VII

1.- Introducción............................................................... 1

1.1.- Breve reseña histórica de las resecciones pancreáticas...................3

1.2.- Patología quirúrgica pancreática y de la región periampular...............5

1.3.- Técnica quirúrgica de la patología pancreática...........................8

1.3.1.- Duodenopancreatectomía cefálica...........................8

1.3.1.1.- Duodenopancreatectomía cefálica con preservación pilórica o sin preservación pilórica.........................9

1.3.1.2.- Abordaje de la arteria primero “Artery-first approach”.10

1.3.1.3.- Linfadenectomía estándar o extendida....................11

1.3.1.4.- Pancreatoyeyunostomía o pancreatogastrostomía........12

1.3.1.5.- Empleo del tutor en la anastomosis pancreática.........13

1.3.1.6.-Utilidad del drenaje abdominal tras DPC................13

1.3.2.- Pancreatectomía distal......................................14

1.3.3.- Enucleación y pancreatectomía central........................17

1.3.4.- Pancreatectomía total.........................................20

1.4.- Complicaciones de la cirugía pancreática..............................22

1.4.1.- Complicaciones globales.................................23

1.4.2.- Complicaciones específicas...............................26

1.4.2.1.- Fístula pancreática postoperatoria.....................26 
1.5.- Escalas predictoras de riesgo de complicación tras cirugía pancreática.....37

1.5.1.- Preoperative pancreatic resection (PREPARE) score............39

1.5.2.- Escala de Wiltberger..................................42

1.6.- Justificación......................................................46

2.- Hipótesis y objetivos.......................................................49

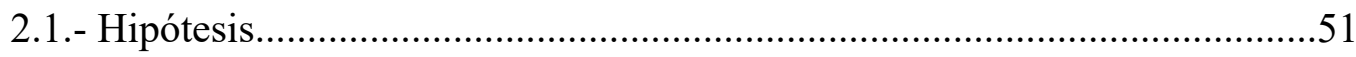

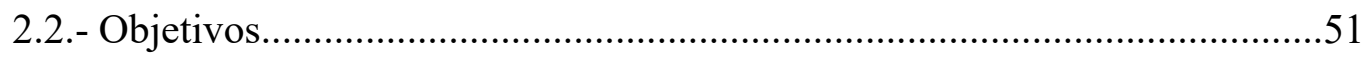

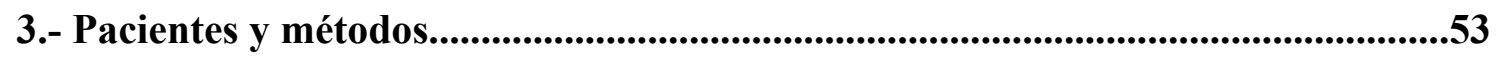

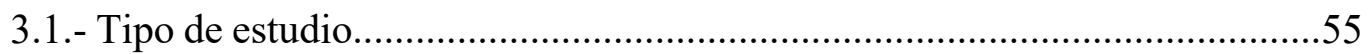

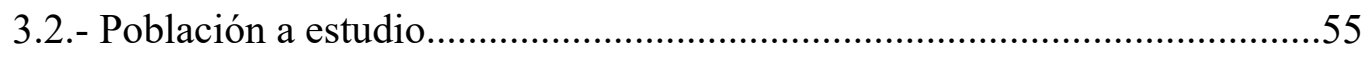

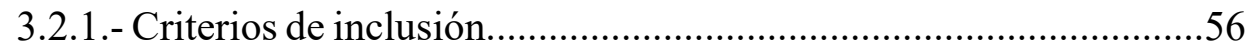

3.2.2.- Criterios de exclusión............................................................56

3.2.3.- Tamaño muestral.....................................................................57

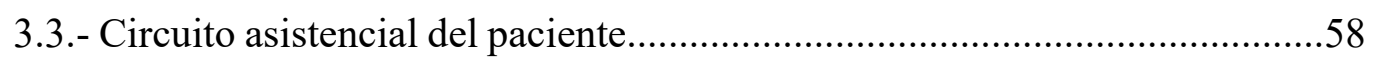

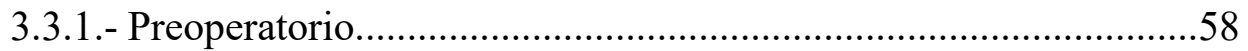

3.3.2.- Preoperatorio inmediato........................................................60

3.3.3.- Técnica quirúrgica..............................................................60

3.3.4.- Protocolo postoperatorio.........................................................62

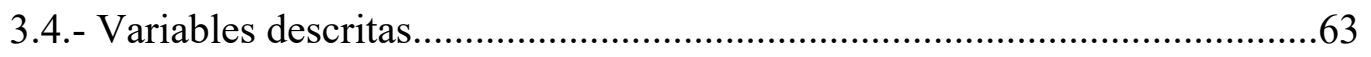

3.4.1.- Variables demográficas...........................................................63

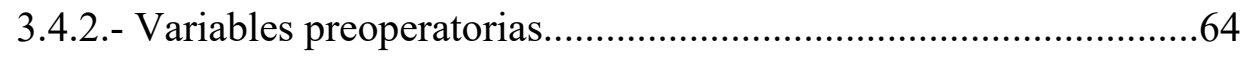

3.4.3.- Variables intraoperatorias.....................................................67

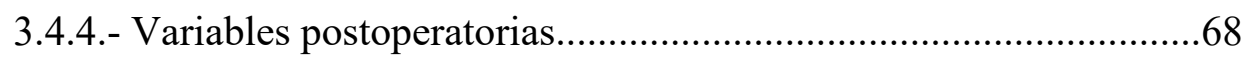


3.5.1.- Recogida y manejo de los datos .69

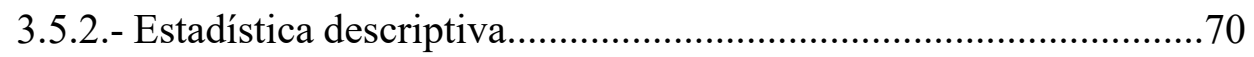

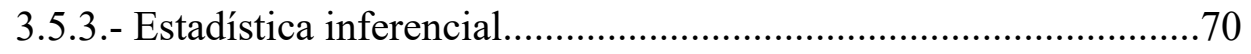

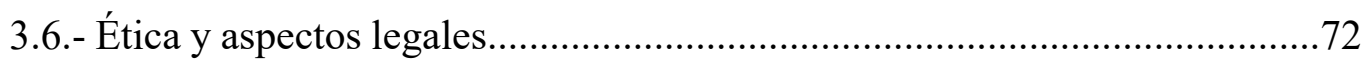

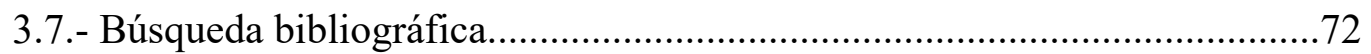

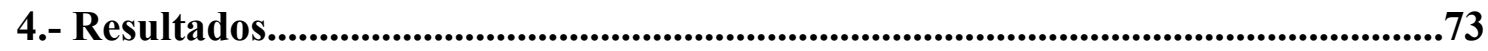

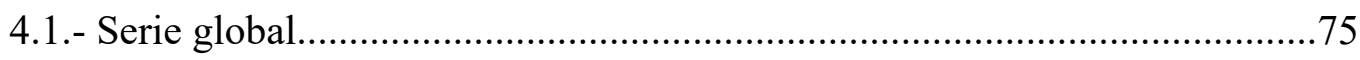

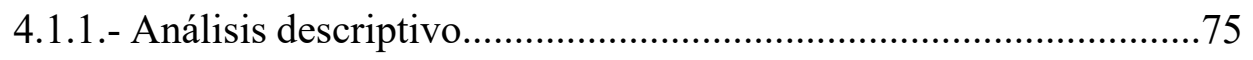

4.1.1.1.- Variables demográficas..............................................75

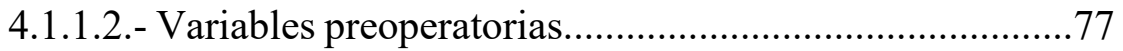

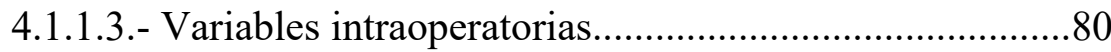

4.1.1.4.- Variables postoperatorias.......................................... 81

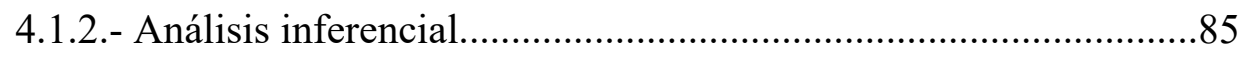

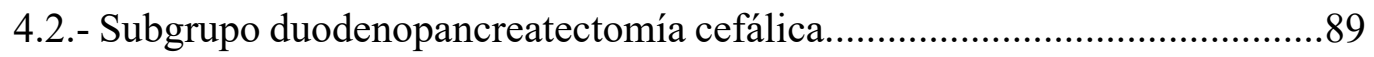

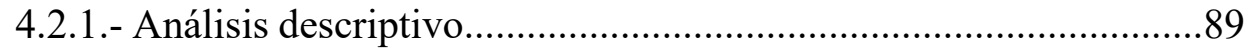

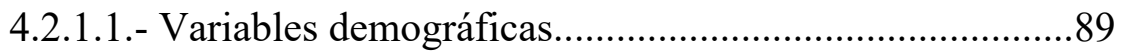

4.2.1.2.- Variables preoperatorias...........................................90

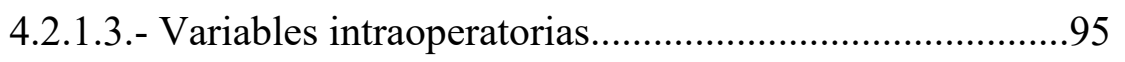

4.2.1.4.- Variables postoperatorias...........................................96

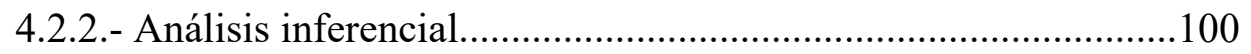

5.- Discusión...........................................................................................................................105

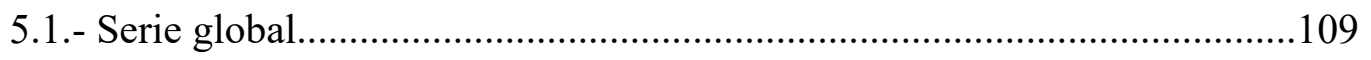

5.1.1.- Análisis de los resultados descriptivos....................................110

5.1.2.- Análisis de la validación de la escala PREPARE ........................126 
5.1.3.- Correlación de la escala PREPARE con otras variables de resultado.

5.2.- Subgrupo duodenopancreatectomía cefálica .132

5.2.1.- Análisis de los resultados descriptivos.

5.2.2.- Análisis de la validación de la escala PREPARE y Wiltberger en el SDPC 150

5.2.3.- Análisis bivariante entre la escala PREPARE y Wiltberger en el SDPC y las variables resultado. 153

5.3.- Integración y aplicabilidad futura 154

5.4.- Limitaciones. .157

5.5.- Control de calidad de la validación externa. 158

6.- Conclusiones.. .161 


\section{ABREVIATURAS}

ASA

American society of Anesthesiologists

AUC

Area Under Curve.

CA 125 Antígeno carbohidrato 125

CA 19-9 Antígeno carbohidrato 19-9

CEA Antígeno carcinoembrionario

DPC Duodenopancreactecomía cefálica

DPO Día postoperatorio

DPT Duodenopancreatectomía total

EPOC Enfermedad pulmonar obstructiva crónica

FPCR Fístula pancreática clínicamente relevante

FPPO Fistula pancreática postoperatoria

g/dL gramo/decilitro

HPP Hemorragia postoperatoria postpancreatectomía

HURH Hospital Universitario Río Hortega

IC 95\% Intervalo de confianza al 95\%

IHQ Infección de herida quirúrgica

IMC Índice de masa corporal

INR International Normalized Ratio

ISGPS International Study Group of Pancreatic Surgery

$\mathrm{Kg} / \mathrm{m}^{2} \quad$ Kilogramos por metro cuadrado

lpm latidos por minuto 


\begin{tabular}{|c|c|}
\hline $\mathrm{mEq} / \mathrm{L}$ & Miliequivalentes por litro \\
\hline $\mathrm{mg} / \mathrm{dL}$ & miligramos/decilitro \\
\hline $\mathrm{mmHg}$ & milímetros de mercurio \\
\hline NPT & Nutrición parenteral total \\
\hline PCR & Proteína $\mathrm{C}$ reactiva \\
\hline PD & Pancreatectomía distal \\
\hline RIQ & Rango intercuartílico \\
\hline ROC & Receiver Operating Characteristic \\
\hline $\mathrm{RR}$ & Riesgo relativo \\
\hline RVG & Retraso del vaciamiento gástrico \\
\hline SDPC & Subgrupo DPC \\
\hline SG & Serie global \\
\hline SIRS & Systesmic Inflammatory Response Syndrome \\
\hline SNG & Sonda nasogástrica \\
\hline TC & Tomografía computerizada \\
\hline TEP & Tromboembolismo pulmonar \\
\hline TPMI & Tumor papilar mucinosa intraductal \\
\hline TVP & Trombosis venosa profunda \\
\hline$\mu l$ & microlitro \\
\hline
\end{tabular}




\section{AGRADECIMIENTOS}

Gracias a Alba, porque sin ti esta Tesis no hubiera sido posible. Tu apoyo en los momentos más difíciles y tu paciencia ante mi trabajo, han hecho hacerme fuerte para conseguir este objetivo, que también es tuyo.

Gracias a mis padres, ya que me dieron la oportunidad de estar donde estoy, su soporte y cariño han hecho factible que esto forme parte de mi vida.

Gracias a Aurora, por haberme inculcado las ganas de superarme. El camino que ha allanado ha servido para facilitar mi travesía.

Gracias a Mario, un gran amigo, por contagiarme una pequeña parte de sus insaciables ganas de mejora y perfeccionismo innato.

Gracias a Baltasar, por enseñarme a cuestionarme todo y hacerme ver que lo más importante de la medicina está en el paciente.

Gracias a David, por ser tan conciso y enseñarme el pragmatismo de la medicina y la cirugía. 


\section{INTRODUCCIÓN}


Francisco Javier Tejero Pintor 


\section{INTRODUCCIÓN}

\section{1.- BREVE RESEÑA HISTÓRICA DE LAS RESECCIONES PANCREÁTICAS}

El páncreas siempre ha sido considerado, por su situación retroperitoneal, un órgano esotérico y poco conocido. Desde el punto de vista histórico, la primera descripción anatómica se atribuye a Herófilo de Calcedonia, hacía el año 300 antes de cristo en la antigua Grecia. Sin embargo, no fue hasta el año 100 D.C. cuando Rufus de Éfeso observó en su estudio con cadáveres, que esta estructura retroperitoneal no tenía cartílago ni huesos, por lo que lo llamó páncreas $($ del griego $p a n=$ todo, $k r e a s=$ carne $)$ $(1,2)$.

La primera pancreatectomía distal (PD), fue realizada en 1882 por Friedrich Trendelenburg. Más tarde, en 1898 Alessandro Codivilla en la ciudad de Imola, cerca de Bolonia, realizó la primera duodenopancreatectomía cefálica (DPC). El paciente falleció a los 28 días después de la cirugía. Posteriormente en 1909, Walther Kausch realizó la primera DPC con supervivencia $(3,4)$. En 1935, Allen O. Whipple, junto con sus colaboradores del Hospital Presbyterian de Nueva York, reunió los conocimientos necesarios para sistematizar la técnica de la DPC. La técnica originaria fue descrita en dos tiempos, en primer lugar, para evitar la obstrucción biliar y digestiva, se realizaba una colecistogastrostomía y gastroenterostomía. Posteriormente, se completaba la DPC tras la recuperación de la ictericia obstructiva del paciente [figura 1] (5). Más tarde, en 1940, Whipple realizó la primera DPC en un solo tiempo tal y como la conocemos hoy en día [figura 2] (6). No fue hasta 1942 cuando Rockey ejecutó la primera 
duodenopancreatectomía total (DPT) (7), seguida por la DPT de Priestley quien poco después concluyó el mismo procedimiento con supervivencia (8).

Figura 1: Técnica original de DPC realizada en 2 tiempos. [Tomado de Whipple et al. (5)]
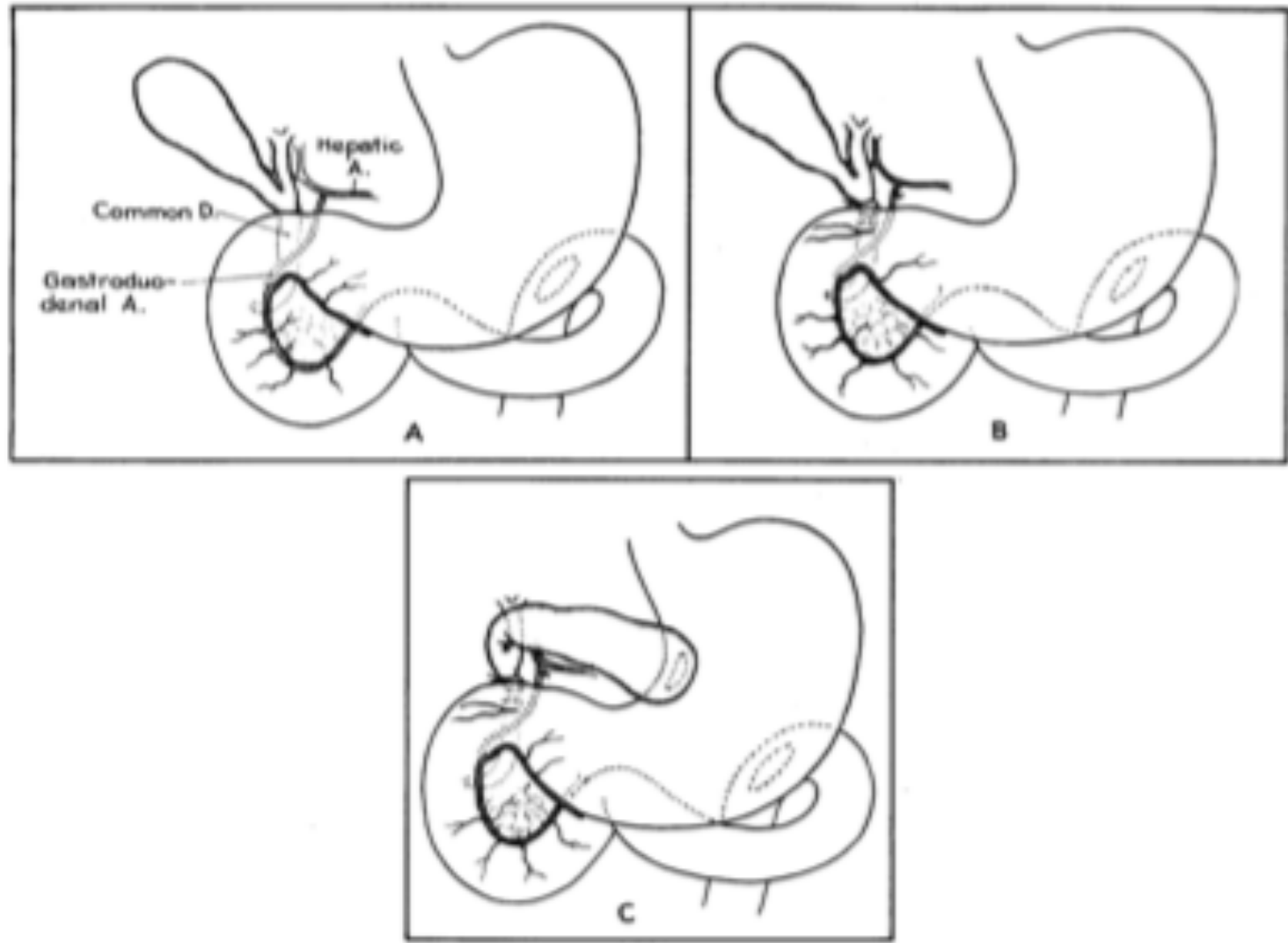

FIRST OPERATION
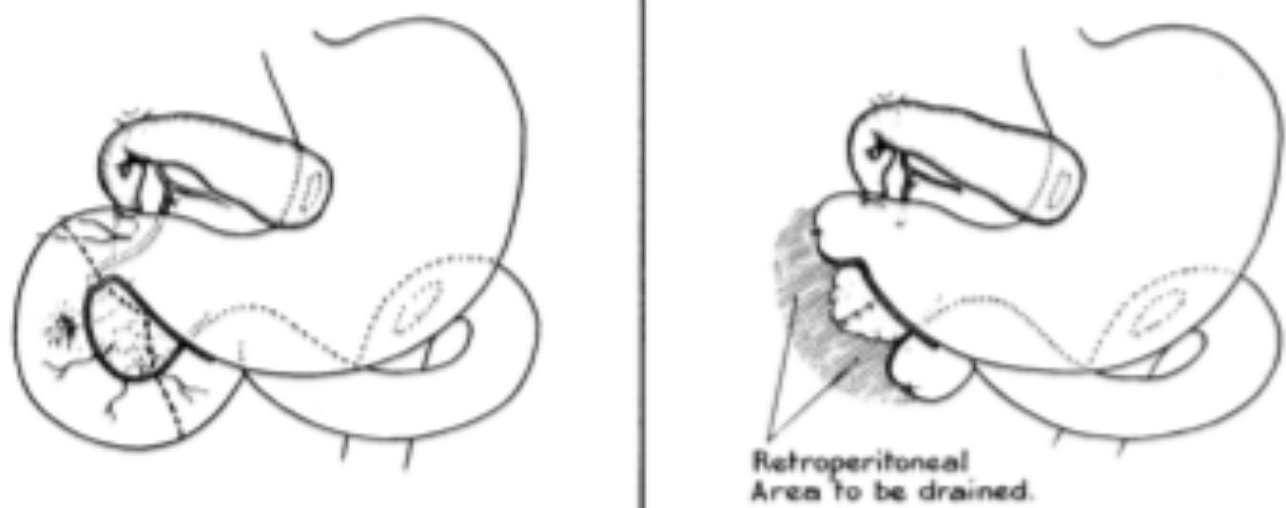

Area to be drained.

END STACE 
Figura 2: Técnica establecida de la DPC en un solo tiempo.

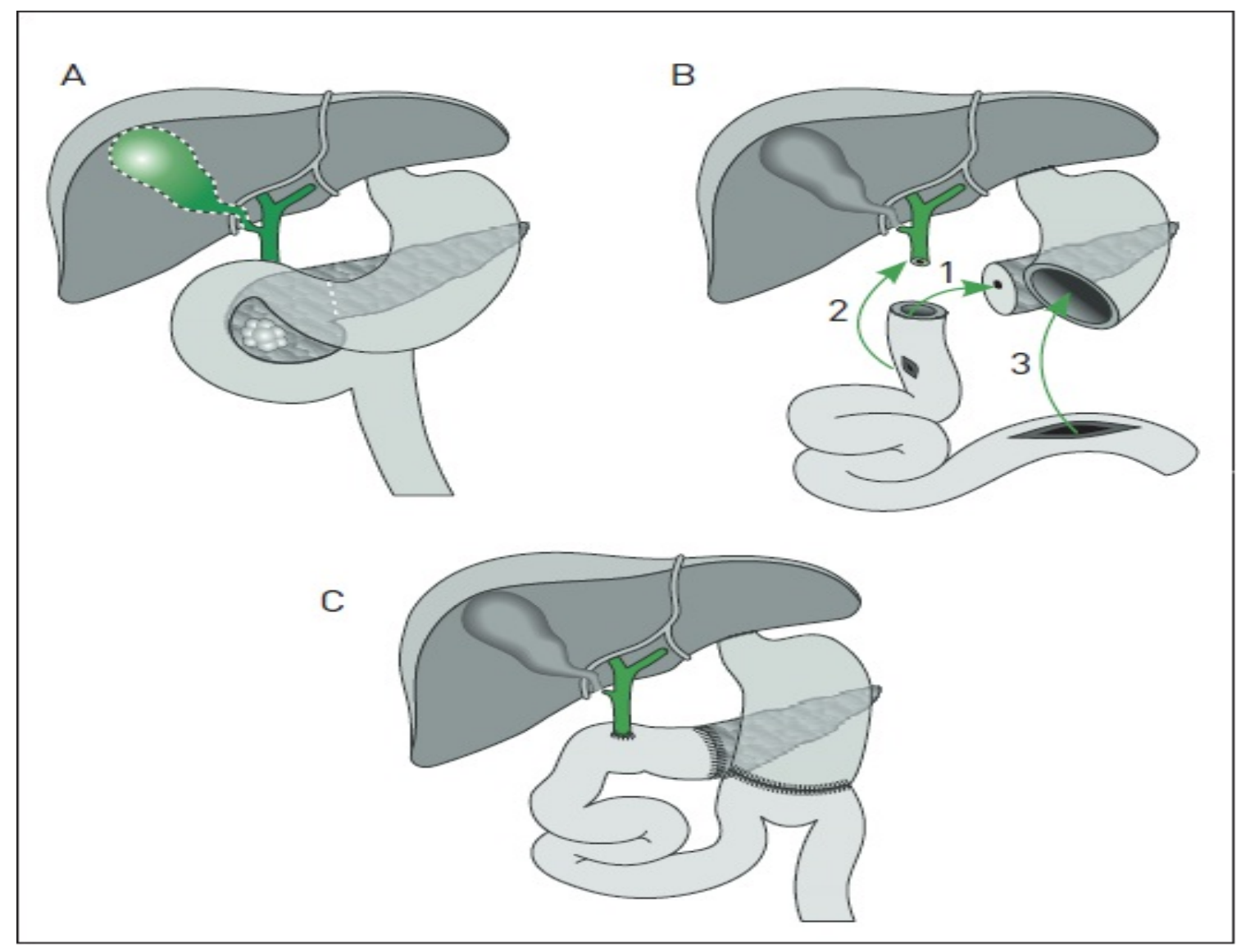

\section{2.- PATOLOGÍA QUIRÚRGICA PANCREÁTICA Y DE LA}

\section{REGIÓN PERIAMPULAR}

La patología quirúrgica pancreática y de la región periampular puede clasificarse en patología benigna y maligna. En la patología benigna destacan, entre las diferentes enfermedades que se incluyen en esta clasificación, los tumores quísticos pancreáticos y la pancreatitis crónica. [Tabla 1]. En la patología maligna resalta el adenocarcinoma de páncreas, aunque en la tabla 2 también se describen otras patologías menos frecuentes. 
Tabla 1: Patología quirúrgica benigna del páncreas y región periampular.

- Lesión precursora de malignidad.

- Epidemiología y localización: aparece en ambos sexos, 60-70 años, más frecuente en cabeza de páncreas, aunque puede afectar a cualquier Tumor parte (9).

papilar - Características: Clasificado en ducto principal pudiendo dilatar el mucinoso Wirsung o de rama secundaria (10)

intraductal - Tratamiento: Resección pancreática en la mayoría de los casos, (TPMI) especialmente si existen factores de riesgo. Ante la ausencia de signos de malignidad existe escasa evidencia para el manejo conservador (10$13)$.

- Lesión precursora de malignidad.

- Epidemiología y localización: más frecuente en mujeres en la quinta década de la vida, localización más común en cuerpo-cola de páncreas

Cistoadenoma (14).

mucinoso - Características: característicamente no se comunica con el Wirsung. Sin embargo, otros autores observaron que este se comunicaba con el ducto pancreático principal hasta en el 30\% de los casos (15).

- Tratamiento: Quirúrgico en la mayoría de los casos (16).

- Lesión benigna muy infrecuentemente maligniza (17).

- Epidemiología y localización: Mujeres sexta-séptima década de la vida, sin localización preferente (18).

Cistoadenoma - Características: El tipo microquístico es el más frecuente. En la seroso tomografía computerizada (TC) Una cicatriz central estrellada en la lesión es muy sugestivo de su diagnóstico (19).

- Tratamiento: Conservador sin seguimiento si tumores pequeños y ausencia de calcificación. Si esto ocurriese se debe realizar seguimiento e incluso resección quirúrgica si aumenta de tamaño (18).

- Lesión precursora de malignidad.

- Epidemiología y localización: Mujeres en la segunda o tercera década

Tumor de la vida, más frecuente en la cola de páncreas (20-22).

pseudopapilar - Características: variante de células claras, pleomórfica u oncocítica sólido $\quad(23)$.

- Tratamiento: Resección quirúrgica en la mayoría de los casos (2325).

- Patología benigna, aunque invalidante.

- Epidemiología: 4 de cada 100000 pacientes (26).

Pancreatitis - Características: Inflamación y resolución del cuadro clínico con crónica recurrencia y destrucción glandular progresiva (27).

- Tratamiento: El tratamiento endoscópico y/o quirúrgico son necesarios cuando el tratamiento médico óptimo no consigue aliviar el dolor o cuando aparecen complicaciones (27). 


\begin{tabular}{|c|c|}
\hline $\begin{array}{c}\text { Adenocarcinoma de } \\
\text { páncreas }\end{array}$ & $\begin{array}{l}\text {-Epidemiología y localización: } 1 \text { a } 10 \text { casos por } 100.000 \text { personas } \\
\text { (28). Supone el } 95 \% \text { de los tumores pancreáticos exocrinos. El } 60- \\
70 \% \text { de los cánceres de páncreas están localizados en la cabeza y } \\
\text { el } 20-30 \% \text { restante en la cola y cuerpo del páncreas }(29) \text {. } \\
\text {-Características: entre el } 15 \%-20 \% \text { de los pacientes son } \\
\text { candidatos a la cirugía. la supervivencia a los cinco años después } \\
\text { de una pancreatectomía con márgenes libres es solo del } 30 \% \text { si los } \\
\text { ganglios estudiados fueron negativos y del } 10 \% \text { si tiene } \\
\text { enfermedad ganglionar presente }(30,31) \text {. } \\
\text {-Tratamiento: quirúrgico, su resecabilidad viene determinada por } \\
\text { la infiltración local o diseminación a distancia }(32,33) \text {. }\end{array}$ \\
\hline Tumor ampular & $\begin{array}{l}\text {-Epidemiología: } 0,2 \% \text { de todos los tumores malignos digestivos } \\
\text { (34). En los varones la incidencia está en aumento (35). } \\
\text {-Características: mejor pronóstico comparado con el de otros } \\
\text { tumores, su supervivencia a } 5 \text { años supera el } 39 \%(36) \text {. } \\
\text {-Tratamiento: DPC con linfadenectomía regional (37-39). Los } \\
\text { pacientes con alto riesgo quirúrgico que no son candidatos a DPC, } \\
\text { se podrían beneficiar de una ampulectomía transduodenal (39). }\end{array}$ \\
\hline Colangiocarcinoma & $\begin{array}{l}\text {-Epidemiología: su aparición supone únicamente el } 3 \% \text { de todos } \\
\text { los cánceres de tracto digestivo con una incidencia } 1-2 \text { casos por } \\
100.000 \text { habitantes (40). } \\
\text {-Características: el colangiocarcinoma distal tiene una } \\
\text { supervivencia a } 5 \text { años tras cirugía del } 50 \%(41) \\
\text {-Tratamiento: resección quirúrgica. El colangiocarcinoma distal } \\
\text { tiene una alta tasa de resecabilidad (41). }\end{array}$ \\
\hline $\begin{array}{c}\text { Tumor } \\
\text { neuroendocrino } \\
\text { pancreático }\end{array}$ & $\begin{array}{l}\text {-Epidemiología: menor de } 1 \text { caso por cada } 100.000 \text { personas por } \\
\text { año y abarca el } 1-2 \% \text { de todos los tumores de páncreas. Aunque } \\
\text { pueden ser diagnosticados a cualquier edad, la cuarta y quinta } \\
\text { década de la vida es la edad más frecuente de presentación }(42,43) \text {. } \\
\text {-Características: pueden secretar multitud de péptidos } \\
\text { hormonales dando como resultado diferentes síndromes clínicos. } \\
\text { Sin embargo, muchos son no funcionantes }(44) \text {. } \\
\text {-Tratamiento: quirúrgico para prevenir la transformación del } \\
\text { tumor a la malignidad y prevenir su diseminación }(45,46) \text {. Los } \\
\text { tumores menores de } 1 \text { centímetro pueden tratarse de manera } \\
\text { conservadora con seguimiento }(47) \text {. }\end{array}$ \\
\hline $\begin{array}{c}\text { Adenocarcinoma de } \\
\text { duodeno }\end{array}$ & $\begin{array}{l}\text {-Epidemiología y localización: } 55,7 \% \text { de los adenocarcinomas de } \\
\text { todo el intestino delgado se localiza en el duodeno. Su localización } \\
\text { más frecuente es la segunda porción duodenal siendo } \\
\text { especialmente raro el bulbo duodenal (48). } \\
\text {-Características: el diagnóstico es tardío debido a que la mayoría } \\
\text { de tumores no producen síntomas hasta que alcanzan un gran } \\
\text { tamaño. El síntoma más frecuentemente relacionado con el inicio } \\
\text { del estudio diagnóstico es el dolor (49). } \\
\text {-Tratamiento: la DPC suele ser necesaria en tumores de segunda } \\
\text { porción. En primera y tercera porción la duodenectomía aislada es } \\
\text { una opción factible (48). }\end{array}$ \\
\hline
\end{tabular}




\section{3.- TÉCNICA QUIRÚRGICA DE LA PATOLOGÍA PANCREÁTICA}

El páncreas se divide en cuatro regiones principales que tienen notable importancia desde el punto de vista quirúrgico, a saber:

- Cabeza: Localizada dentro de la concavidad del duodeno y formada por las tres primeras porciones del duodeno.

- Proceso unciforme: posterior a los vasos mesentéricos superiores.

- Cuello: anterior a los vasos mesentéricos superiores (arteria mesentérica superior y vena mesentérica superior). Posterior a él se sitúa la vena porta.

- Cuerpo: continúa posterior al estómago hacia la izquierda y ascendiendo ligeramente.

- Cola: termina tras pasar entre las capas del ligamento esplenorrenal. Es La única parte del páncreas intraperitoneal.

\subsection{1.- DUODENOPANCREATECTOMÍA CEFÁLICA}

La DPC también denominado procedimiento de Whipple es una técnica quirúrgica compleja y con alto riesgo de morbilidad intra y postoperatoria. Por ello, los mejores resultados en cuanto a mortalidad y de supervivencia a largo plazo, los tienen los centros hospitalarios con alto volumen (50).

La indicación más frecuente para realizar este tipo de cirugía es la presencia de un tumor maligno o premaligno en la cabeza del páncreas o en alguna de las estructuras periampulares (vía biliar principal, ampolla de Vater o segunda porción duodenal). En 
ocasiones, si un tumor benigno aparece adyacente a dicha zona también puede ser necesaria su exéresis (51). La DPC convencional está constituida por dos fases. En la primera parte de la cirugía, la llamada fase de resección, implica la exéresis de la cabeza del páncreas, el duodeno, los primeros $10-15 \mathrm{~cm}$ del yeyuno, la vía biliar principal, la vesícula biliar y la mitad distal del estómago. En la segunda fase, se debe realizar la reconstrucción para un asa alimentaria y derivación pancreatobiliar. En este sentido, en los últimos años han aparecido una serie de modificaciones a la técnica original (6), con el objetivo de disminuir la morbilidad. Los aspectos técnicos de las mismas se muestran en los siguientes epígrafes:

\subsubsection{1.- DUODENOPANCREATECTOMÍA CEFÁLICA CON PRESERVACIÓN PILÓRICA O SIN PRESERVACIÓN PILÓRICA}

La DPC con preservación pilórica fue inicialmente descrita con el objetivo de disminuir el síndrome de dumping tras la gastrectomía y mejorar la función gastrointestinal fisiológica aportando un beneficio potencial a la digestión y al estado nutricional a largo plazo (52). Surgieron entonces varios ensayos clínicos aleatorizados y no aleatorizados apoyando la realización de la DPC con preservación pilórica para mejorar el retraso del vaciamiento gástrico (RVG) (53-56). Por otro lado, un ensayo clínico aleatorizado (57) y seis no aleatorizados (58-63), concluían que la DPC con resección pilórica mejoraba el RVG. Debido a la controversia generada, seis metanálisis recientemente publicados han puesto de manifiesto por unanimidad que la DPC con resección pilórica es la técnica requerida para mejorar mencionada complicación $(59,64-$ $68)$. 


\subsubsection{2.- ABORDAJE DE LA ARTERIA PRIMERO}

"ARTERY-FIRST APPROACH"

Una posible contraindicación, para la mayoría de los grupos que realizan este tipo de cirugía, es la infiltración de la arteria mesentérica superior, porque si la vena mesentérica superior está infiltrada esta puede ser habitualmente resecada, aunque con dificultad técnica (69). El abordaje de la arteria en primer lugar es útil en pacientes en los que se sospecha una arteria hepática derecha anómala o una arteria hepática accesoria con origen en la arteria mesentérica superior. Este tipo de abordaje también tiene como objetivo afrontar la temprana evaluación de la resecabilidad, especialmente en aquellos que han recibido quimioterapia o radioterapia neoadyuvante (70).

La primera descripción técnica del abordaje de la arteria en primer lugar fue realizada por Pessaux et al. con un abordaje posterior (71). Posteriormente diferentes modificaciones técnicas fueron publicadas: abordaje anterior o inferosupracólico (72), combinación de abordaje anterior y posterior "maniobra de Hanging" (73), abordaje infracólico (73), abordaje derecho o medial (73), abordaje posteroizquierdo (74) y abordaje superior (70) [Figura 3]. Aunque el cirujano debe conocer los diferentes abordajes para abordar la arteria mesentérica superior, la evidencia científica muestra que aunque está técnica se realice de rutina en algunos centros especializados, se debe utilizar únicamente en las situaciones anteriormente descritas (70). 
Figura 3: Vías de abordaje de la arteria en primer lugar. S-Superior, A-Anterior, PPosterior, L-Posteroizquierdo, R-Derecho o medial, M-Infracólico. [Tomado de Ironside et al. (75)].

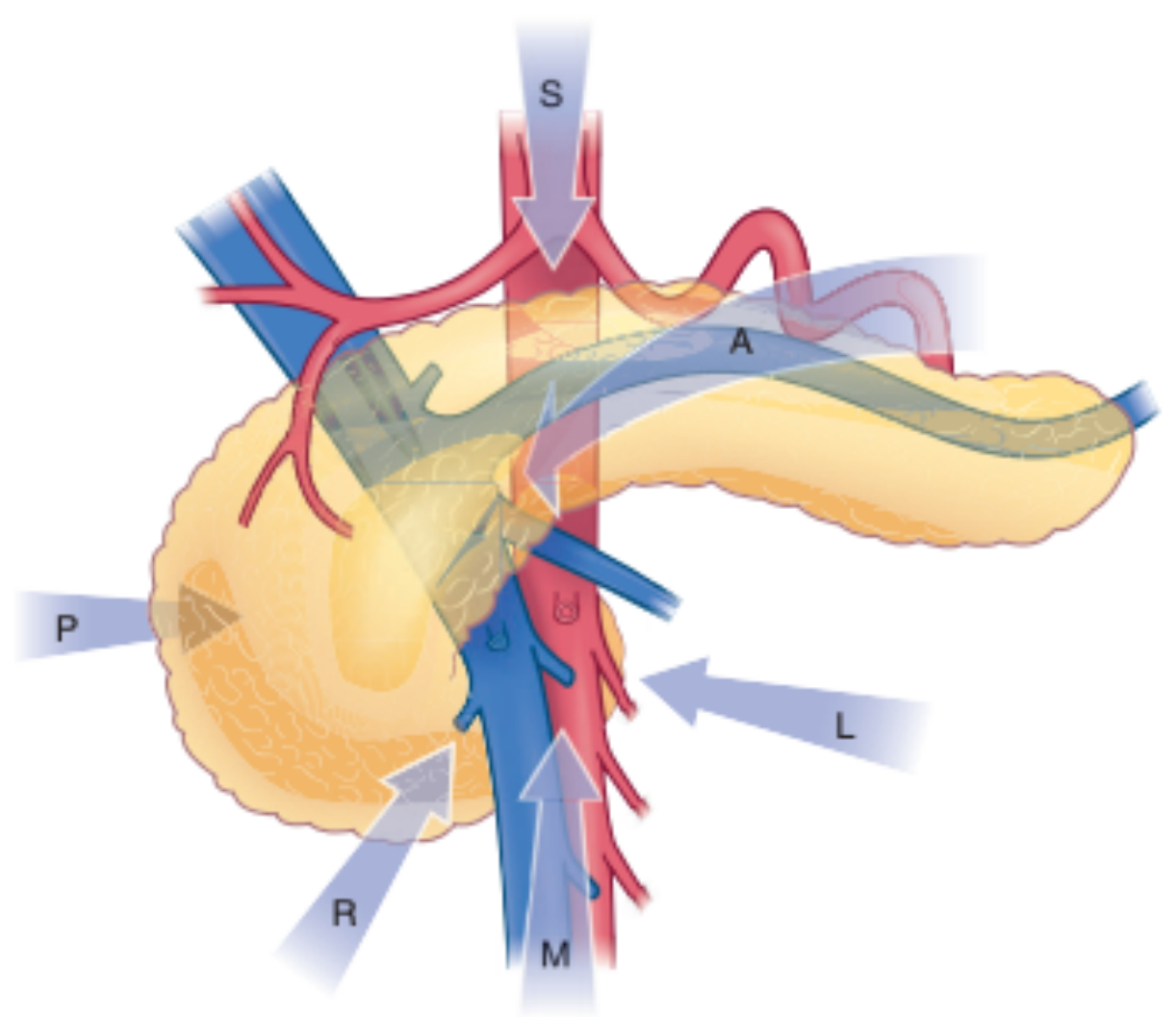

\subsubsection{3.- LINFADENECTOMÍA ESTÁNDAR O EXTENDIDA}

La linfadenectomía estándar de la DPC se caracteriza por abarcar en su exéresis las estaciones ganglionares 5, 6, 8a, 12b1, 12b2, 12c, 13a, 13b, 14a,14b, 17a y 17b [Figura 4] (76). Algunos grupos, principalmente japoneses, realizan de manera sistemática linfadenectomía extendida (incluyendo todos los grupos ganglionares 8, 9, 12, 14 y el 16a2 y 16b1). Sin embargo, el papel de la linfadenectomía extendida es controvertido, ya que algunos ensayos sugieren peor calidad de vida sin aumentar la supervivencia global $(77,78)$. 
Figura 4: Estaciones ganglionares del drenaje linfático del cáncer de cabeza de páncreas. [ Tomado de Japan pancreas society (79)].

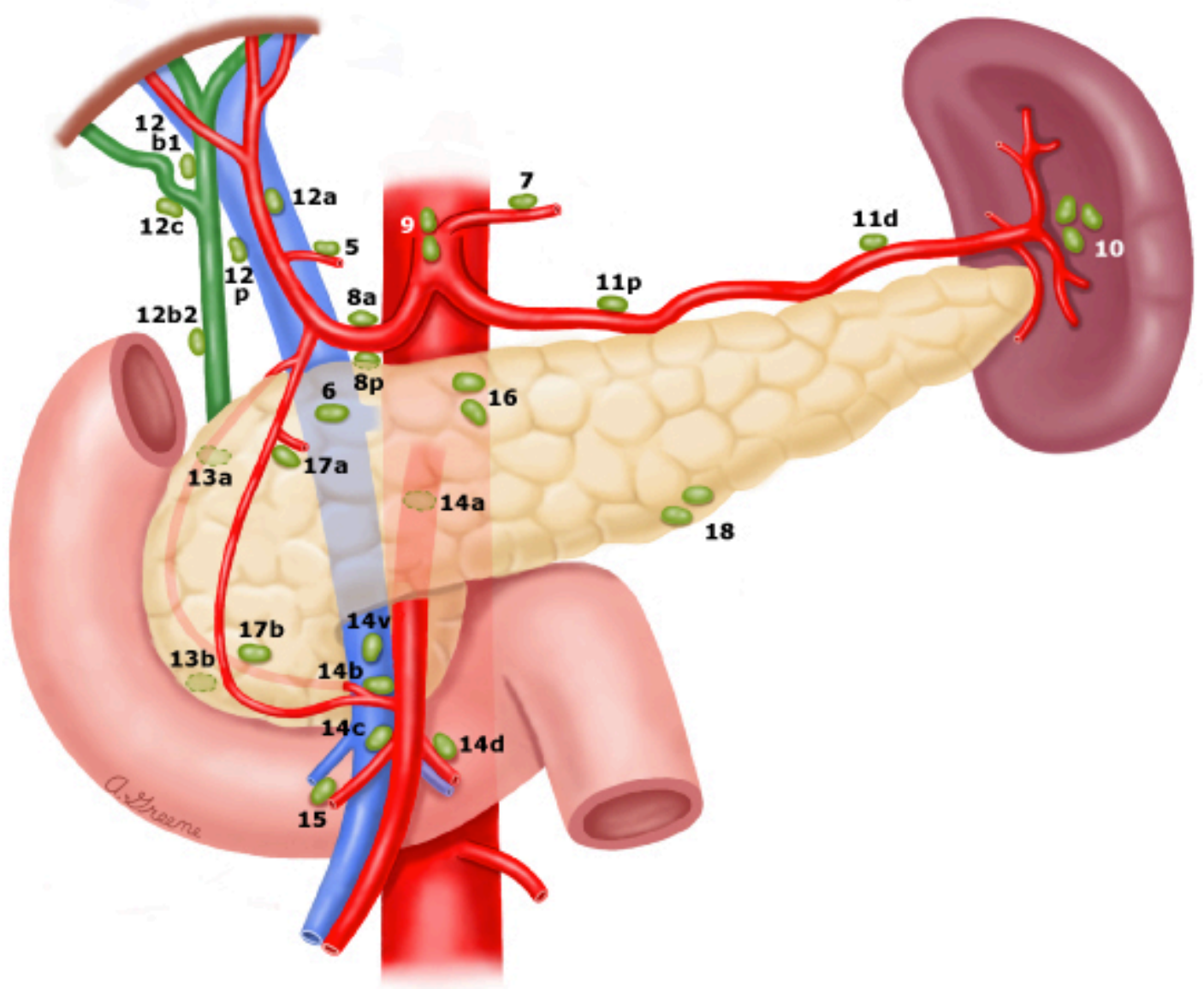

\subsubsection{4.- PANCREATOYEYUNOSTOMÍA O}

\section{PANCREATOGASTROSTOMÍA}

En los últimos años este tema ha suscitado debate, en relación a la fístula pancreática postoperatoria (FPPO), en multitud de reuniones científicas. En 2014 Clerveus et al, publicaron un metanálisis concluyendo que no había diferencias significativas entre ambas técnicas en términos de morbilidad, tasas de reoperación y mortalidad (80). En los años 2017 y 2018, dos metanálisis confirmaron la falta de evidencia que tiene realizar una técnica sobre otra $(81,82)$, además en uno de ellos, la 
pancreatogastrostomía demostró tener una mayor incidencia de hemorragia postoperatoria postpancreatectomía (HPP) con respecto a la pancreatoyeyunostomía (81).

\subsubsection{5.- EMPLEO DEL TUTOR EN LA ANASTOMOSIS PANCREÁTICA}

El empleo del tutor de la anastomosis pancreática ha sido investigado con el objetivo de disminuir el potencial riesgo de FPPO o disminuir su gravedad. La utilidad de tener una guía que permita comunicar el débito pancreático con la luz intestinal es interesante desde el punto de vista teórico en las anastomosis ductomucosa. No existe evidencia que confirme el beneficio el uso de estos tutores para disminuir el riesgo de FPPO (83).

\subsubsection{6.- UTILIDAD DEL DRENAJE ABDOMINAL TRAS DPC}

Varios metanálisis recientemente publicados demostraron la utilidad del drenaje abdominal para disminuir el riesgo de complicaciones (absceso intraabdominal o mortalidad) tras DPC (84-87). Por otro lado, un ensayo clínico alemán (PANDRA), ha concluido mayores tasas de reintervención y FPPO en el grupo de pacientes con drenaje (88). Según la última actualización de las guías del International Study Group of Pancreatic Surgery (ISGPS), se recomienda no utilizar ningún dispositivo de drenaje en aquellos pacientes con un grado de riesgo bajo o moderado de FPPO, únicamente reservando el uso de drenaje intraabdominal para aquellos pacientes con alto riesgo de complicación. Se mantiene la recomendación de realizar una determinación de amilasa en el líquido de drenaje el tercer día postoperatorio (DPO), si esta es tres veces menor al 
límite alto de la normalidad del laboratorio de referencia, se descarta, en principio, la ausencia de FPPO (83).

\subsection{2.- PANCREATECTOMÍA DISTAL}

Existe una variedad de patologías pancreáticas, malignas y benignas que pueden cumplir criterios para realizar una PD. En el caso de patologías malignas o premalignas que precisan una resección de tipo PD se encuentran: el adenocarcinoma de cola de páncreas, neoplasias quísticas, tumores neuroendocrinos, y de forma excepcional las metástasis únicas en la glándula pancreática. La patología benigna susceptible de resección pancreática incluye: pancreatitis crónica, pseudoquiste pancreático y traumatismo pancreático. Esta resección pancreática, la mayoría de las veces se realiza seccionando a la izquierda de la arteria mesentérica superior y vena mesentérica inferior. En una serie de 232 PD publicada por Goh et al (89), 164 de ellas fueron realizadas por enfermedades propias del páncreas y el $84 \%$ fueron malignas o premalignas.

La técnica clásica de la PD es la resección pancreática de izquierda a derecha. Las variantes quirúrgicas de la $\mathrm{PD}$ son la pancreatoesplenectomía radical anterógrada modular y la pancreatectomía distal con resección del tronco celiaco. La primera de ellas fue descrita con la intención de aumentar los márgenes de resección pancreática y ampliar las posibilidades de que se obtenga un borde tumoral negativo en el estudio de la pieza quirúrgica. La resección del plano posterior de la fascia renal anterior es denominada pancreatoesplenectomía radical anterógrada modular anterior, esta puede ser necesaria cuando el tumor no ha penetrado la cápsula pancreática posterior. La resección del plano posterior de la glándula suprarrenal y de la fascia de Gerota es denominado como 
pancreatoesplenectomía radical anterógrada modular posterior, y puede ser necesario cuando el tumor ha superado la cápsula pancreática posterior (90) [Figura 5].

La pancreatectomía distal con resección del tronco celiaco es una técnica agresiva, pero que puede tener su utilidad en los tumores adyacentes al tronco celiaco. Anatómicamente, el aporte sanguíneo hepático proviene de la arteria hepática propia. Sin embargo, el aporte hacía el hígado retrogradamente desde la arteria pancreatoduodenal inferior a través de la arteria gastroduodenal, en ocasiones es suficiente para su irrigación. Por eso, la sección del tronco celiaco y de la arteria hepática propia en bloque con una pancreatectomía distal, es útil para tumores en dicha localización, sin observar problemas en el suplemento sanguíneo hepático (90) [Figura 6]. En una muy reciente revisión retrospectiva europea en 68 pacientes de 20 hospitales en 12 países, la serie más larga en países occidentales hasta el momento, obtuvieron un 25\% de complicaciones graves, (3 pacientes tuvieron isquemia gástrica), un 21\% de FPPO B/C y 16\% de mortalidad (91).

Figura 5: Planos del margen posterior y dirección de la disección de los diferentes tipos de PD, [tomado de Strasberg et al. (90)]

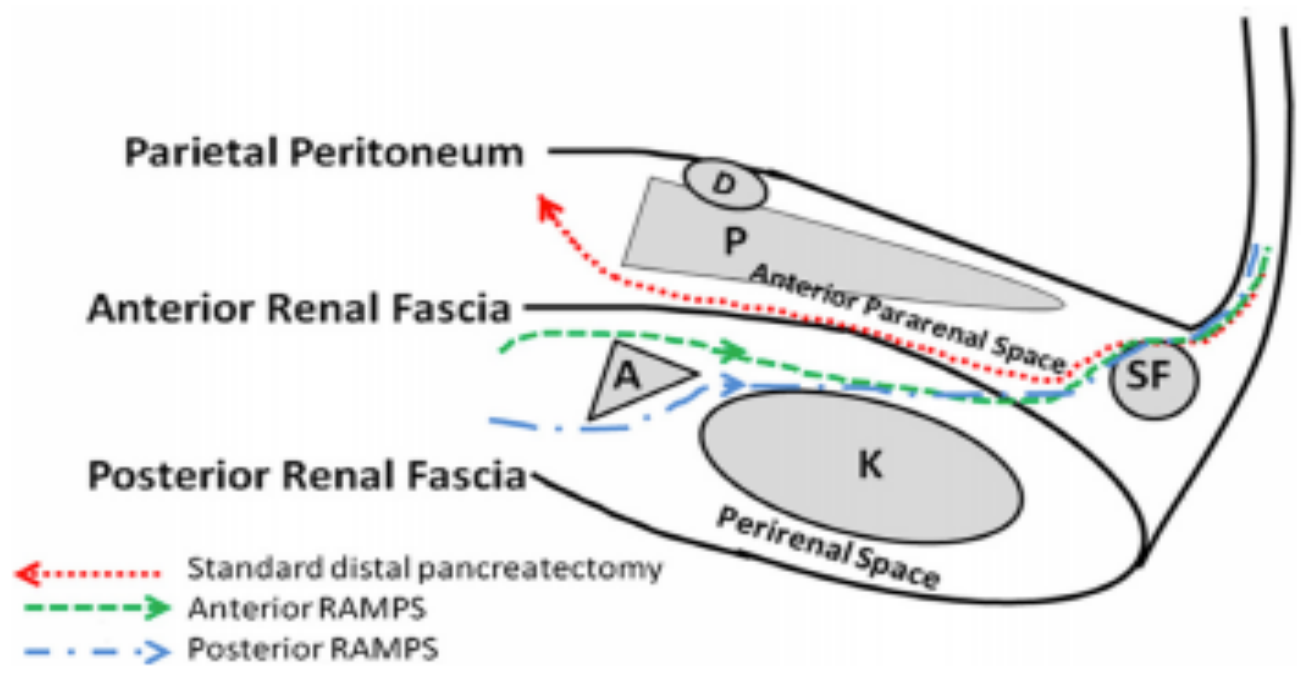


Figura 6: Vías colaterales de aporte sanguíneo hepático (flechas), zona de transección en la pancreatectomía distal con resección del tronco celiaco (línea discontinua), [tomado de Strasberg et al. (90)].

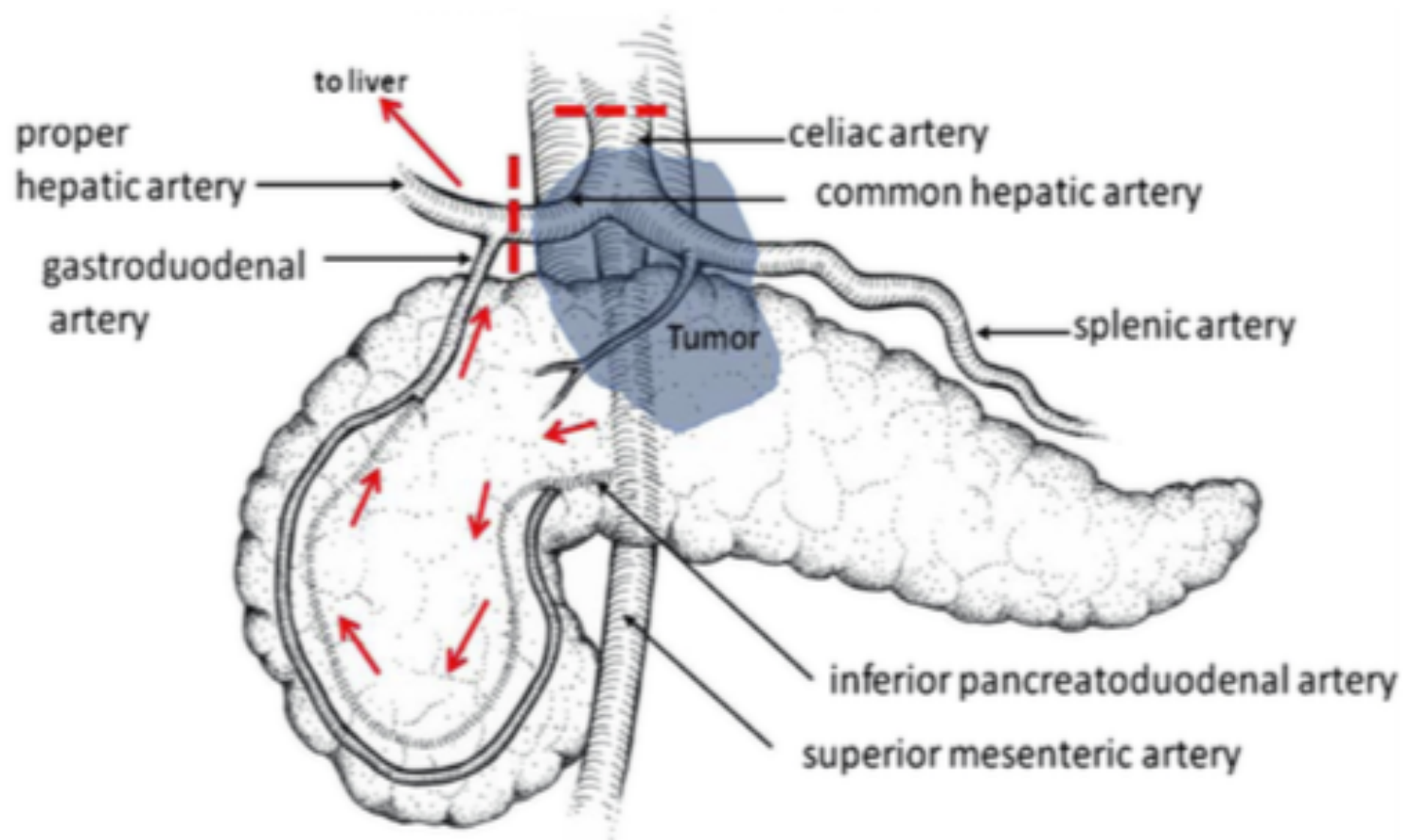

El abordaje para realizar la PD puede ser laparoscópico, robótico o por vía abierta. En un metanálisis publicado en 2016 sobre estudios observacionales, la PD laparoscópica se ha asociado con una disminución de la estancia hospitalaria postoperatoria. Sin embargo, al tratarse de estudios observacionales no aleatorizados, esta diferencia pudo ser debida a la diferencia de estadiaje entre la vía de abordaje de ambas técnicas (92). Actualmente, parece que en todos los campos quirúrgicos la tendencia es ir hacía la cirugía mínimamente invasiva. En un metanálisis que comparaba la PD con abordaje por robot frente al abordaje laparoscópico, no encontró diferencias significativas que apoyen uno u otro abordaje (93). Sin embargo, otro metanálisis recientemente publicado sugiere que aunque el abordaje por robot tiene mayor coste que el laparoscópico, mejora la tasa de preservación esplénica, disminuye la tasa de conversión y reduce la estancia postoperatoria (94). 
Cuando es necesaria una $\mathrm{PD}$, se realiza en la mayoría de las veces una esplenectomía asociada para obtener mayor número de ganglios y asegurar que los márgenes estén libres de tumor. La primera PD con preservación esplénica fue descrita por Mallet-Guy y Vachon en 1943 (95). Ésta es la técnica más clásica. Consiste en dividir los vasos del páncreas y el bazo llegando hasta e plano posterior del páncreas, donde se identifican y se ligan los vasos pancreáticos provenientes de la vena y arteria esplénica. Posteriormente, en 1988 Warshaw describió otra técnica de preservación esplénica, que consiste en ligar los vasos esplénicos, dejando el bazo únicamente irrigado por los vasos gástricos cortos y los gastroepiploicos izquierdos (96). La preservación esplénica puede llevarse a cabo si el tumor causante de la lección es un tumor neuroendocrino pequeño, normalmente benignos o lesiones quísticas premalignas. Una revisión sistemática de estudios observacionales, concluyó que la preservación esplénica mejoraba la incidencia de abscesos intraabdominales, sin aumentar la tasa de sangrado postoperatorio, FPPO, infección de herida quirúrgica, ni trombosis vascular esplénica (97).

\subsection{3.- ENUCLEACIÓN Y PANCREATECTOMÍA CENTRAL}

Tanto los tumores benignos como las lesiones quísticas o los tumores neuroendocrinos, no necesariamente requieren una resección extensa del parénquima pancreático para conseguir su exéresis. La enucleación es una técnica quirúrgica que consiste en realizar resecciones limitadas y que, además, disminuye el riesgo de insuficiencia pancreática postoperatoria (98). La enucleación no debería realizarse cuando la lesión es grande o cuando la lesión está muy próxima a la vía biliar principal o al ducto pancreático, ya que la agresión quirúrgica pudría lesionarlo, dando como resultado una FPPO. 
Los tumores endocrinos, cistoadenoma seosos, cistoadenomas mucinosos, tumores pseudopapilares sólidos o cualquier otra lesión benigna, son subsidiaras de ser indicación para enucleación pancreática. Sin embargo, de acuerdo a las guías del consenso internacional del manejo de los TPMI (11), la identificación de una tumoración maligna en el ducto pancreático principal oscila en un $70 \%$ de los casos. Por lo que, en estos pacientes en los que existen tumores benignos con invasión mayor de $2 \mathrm{~mm}$, se debería reconsiderar otro tipo de cirugía diferente a la enucleación.

Como se ha descrito previamente, la FPPO es esencialmente la complicación más frecuente en el postoperatorio de este tipo de cirugía. Briggs et al. (99) analizaron los resultados de enucleaciones pancreáticas laparoscópicas de 100 pacientes. Los autores concluyeron que existía un $47 \%$ de complicaciones postoperatorias y un $29 \%$ de FPPO. Sin embargo, no existía mortalidad. Por otra parte, Zhou et al. (100) recientemente han publicado los resultados de un metanálisis que incluyó 27 estudios y 1300 pacientes a los que se les realizaron enucleación pancreática. La FPPO fue la complicación más frecuentemente observada apareciendo en un 38\% de los pacientes incluidos, de los cuales el 55\% fue FPPO clínicamente relevante.

Por otro lado, la pancreatectomía central es una opción quirúrgica, cuyo objetivo es la división del parénquima pancreático entre la cabeza y la cola de páncreas, resecando el cuerpo. La técnica consiste en clausurar la parte distal de la cabeza pancreática realizándose una anastomosis entre la parte proximal del remanente de la cola pancreática y el tracto gastrointestinal (en un asa de yeyuno o cara posterior gástrica) [Figura 7] (101). 
Figura 7: A. Técnica de pancreatectomía central. B. Reconstrucción en Y de roux.

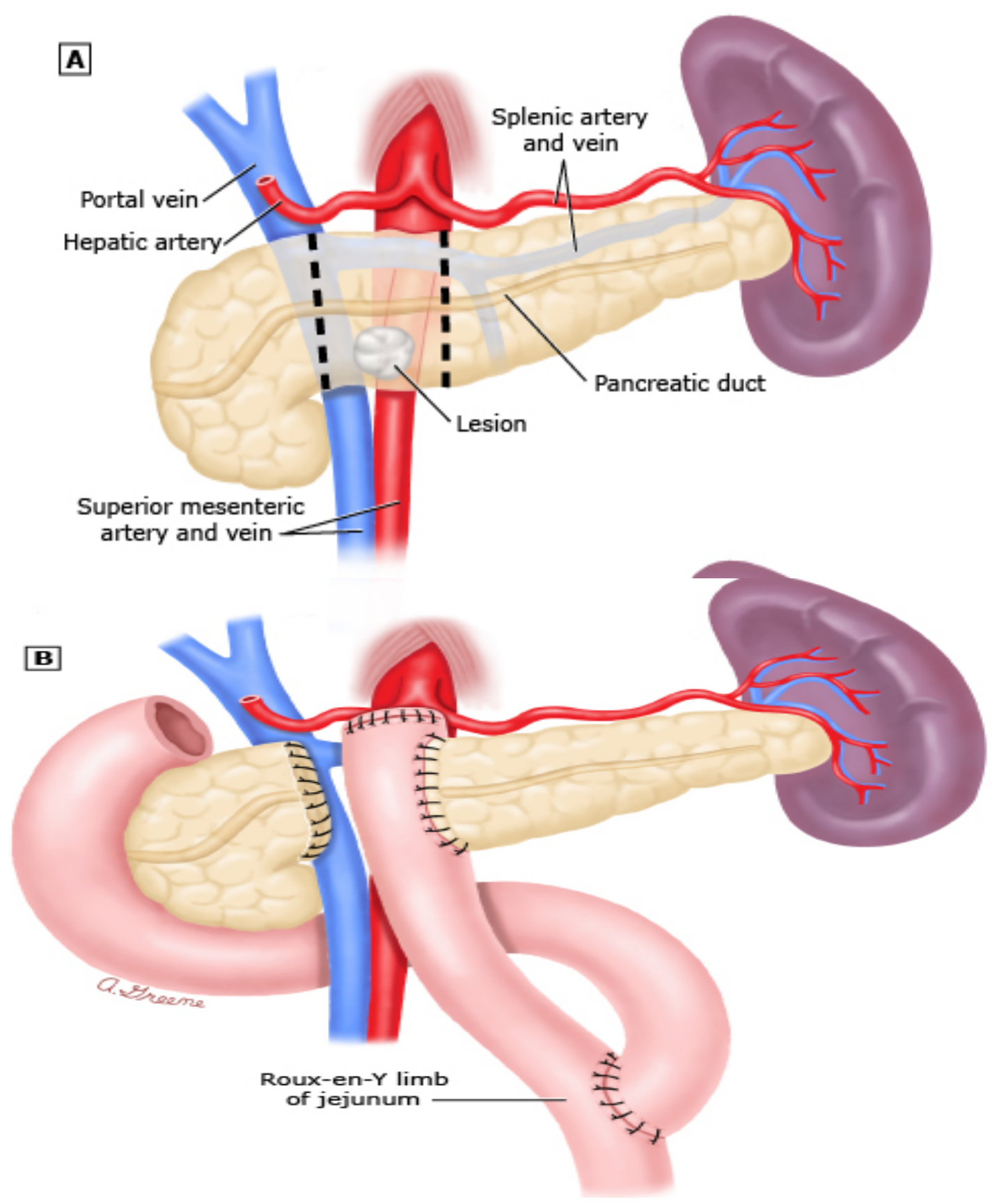

La indicación de la pancreatectomía central es similar a la de la enucleación pancreática, siendo la enucleación más frecuentemente utilizada para lesiones benignas cuya resección no tiene por qué ser necesariamente grande, limitando el papel de la pancreatectomía central para las lesiones malignas (102). Según una revisión sistemática 
que analizó 94 estudios y que incluía 963 pacientes comparando los resultados de pancreatectomía central contra la pancreatectomía distal (103), la primera técnica se asoció con un mayor porcentaje de morbilidad postquirúrgica y mayor incidencia de FPPO pero con menor incidencia de insuficiencia endocrina.

\subsection{4.- PANCREATECTOMÍA TOTAL}

En la década de los 60, la DPT fue considerada como una técnica útil para evitar el riesgo de FPPO y además conseguir una ampliación de bordes libre de tumor. Posteriormente, las secuelas postoperatorias después de este procedimiento como es la diabetes de muy difícil control, la malabsorción o la esteatorrea, hicieron abandonar en gran medida esta técnica para estos fines.

Actualmente, la DPT, a pesar de ser una cirugía más extensa y debido a la mejora de las medidas preoperatorias, perioperatorias y postoperatorias, puede ser considerada con el mismo riesgo quirúrgico que tiene una DPC (104). Algunos autores, han demostrado la equiparación de niveles de $\mathrm{HbA} 1 \mathrm{c}$ entre los pacientes diabéticos tipo 1 y a los que se les ha realizado una DPT (105).

Las indicaciones de la DPT se pueden observar en la tabla 3. En conjunto se reserva esta técnica para aquellas lesiones con un gran potencial de mortalidad que no pueden ser resueltas por medidas conservadoras o técnicas quirúrgicas menos agresivas. 
Tabla 3: Indicaciones de DPT (105).

\begin{tabular}{|c|c|c|}
\hline $\begin{array}{c}\text { Patologías benignas } \\
\text { graves }\end{array}$ & Patologías premalignas & Patologías malignas \\
\hline $\begin{array}{l}\text { - Pancreatitis } \\
\text { crónica. } \\
\text { - Pancreatitis } \\
\text { hereditaria. } \\
\text { - Trauma. }\end{array}$ & - $\quad$ TPMI & $\begin{array}{l}\text { - Adenocarcinoma } \\
\text { de páncreas } \\
\text { - } \begin{array}{l}\text { Tumor } \\
\text { neuroendocrino } \\
\text { pancreático }\end{array} \\
\text { - } \begin{array}{l}\text { Metástasis de } \\
\text { cáncer de células } \\
\text { renales }\end{array}\end{array}$ \\
\hline
\end{tabular}

En ocasiones la decisión de realizar la DPT es tomada intraoperatoriamente para prevenir la FPPO en pacientes con un parénquima muy blando o cuando los márgenes de resección son positivos en el estudio de la pieza quirúrgica. Otras veces, la decisión de completar la pancreatectomía durante el postoperatorio es debido a la aparición de complicaciones resultantes de una resección pancreática parcial. En el análisis de 136 pacientes con DPT presentado por Almond et al, el 82\% tuvieron una DPT planeada. Al $11 \%$ de los pacientes le realizaron la conversión a DPT de una DPC y el 7\% completaron la DPT durante el postoperatorio (106).

Existen diferentes alternativas técnicas para la DPT. La preservación esplénica o duodenal en esta cirugía fue descrita por primera vez en pacientes que requerían una DPT por pancreatitis crónica y en los que no se les quiere aumentar la morbimortalidad siendo una causa no tumoral, por lo que no es necesario tener márgenes amplios.

La DPT con preservación esplénica es útil en paciente con pancreatitis crónica por lo anteriormente descrito. Sin embargo, la vena esplénica puede estar íntimamente 
adherida al páncreas debido a la intensa inflamación y fibrosis local, dificultando de esta manera su disección y ligadura. Warshaw describió esta técnica, acuñada con su nombre, en la que se divide la vena y la arteria, preservando los vasos cortos (96). Como se ha mencionado anteriormente en el apartado de PD.

La DPT con preservación duodenal, también principalmente utilizada en pacientes con pancreatitis crónica, aunque no se realiza ninguna discontinuidad en el tracto gastrointestinal, es necesario realizar una reconstrucción en Y de Roux con el objetivo de prevenir la estenosis del conducto biliar o duodenal (107).

La DPT mínimamente invasiva está cada vez más en auge. Aunque este abordaje lleva practicándose desde hace dos décadas, la cirugía abierta para esta técnica es el patrón de oro. En una de las series más largas publicadas hasta la fecha de 5 DPT robóticas, tuvieron una complicación grave sin mortalidad (108).

\section{4.- COMPLICACIONES DE LA CIRUGÍA PANCREÁTICA}

Las resecciones pancreáticas son procedimientos quirúrgicos complejos, por lo que tienen un riesgo no despreciable de complicaciones postoperatorias. En los últimos años, la mejoría de las técnicas quirúrgicas y el manejo perioperatorio global del paciente, así como la experiencia acumulada en centros de alto volumen, ha reducido la morbilidad y mortalidad asociada a este tipo de intervención. Sin embargo, hoy en día la morbilidad global postoperatoria aún se sitúa en torno 30-70\% (109-111). Las complicaciones tras una pancreatectomía son muy variadas e incluyen, no solo complicaciones médicas y quirúrgicas similares a las sufridas en cualquier postoperatorio de cirugía abdominal 
mayor, sino también un grupo específico directamente relacionado con estos procedimientos que será descrito más adelante.

Las complicaciones infecciosas, particularmente la infección de herida o los abscesos intraabdominales, son las más frecuentes después de este tipo de cirugía (alrededor de un 35\%) (112). Otra complicación común en el postoperatorio de cirugías abdominales y particularmente oncológicas, es la aparición de trombosis venosa profunda (TVP) y tromboembolismo pulmonar (TEP). La cirugía conlleva relajación muscular durante el procedimiento y posteriormente el paciente descansa tumbado en la cama durante el postoperatorio, esto asociado a otros factores de riesgo como la obesidad, cirugía oncológica o los antecedentes de TVP/TEP previos hacen que sea más frecuente su diagnóstico durante el postoperatorio inmediato. Diferentes publicaciones han señalado que la incidencia de TVP durante el postoperatorio de cirugía pancreática ocurre entre el 1,5\%-7,2\% de los pacientes

\subsection{1.- COMPLICACIONES GLOBALES}

Existen multitud de publicaciones que concluyen que la cirugía pancreática tiene una gran tasa de complicaciones, y sostienen que este tipo de intervenciones, particularmente en pacientes de alto riesgo con morbilidad asociada, deberían ser realizadas en centros con alto volumen para disminuir esta morbimortalidad postoperatoria. Según el grupo de Teh et al. (113) la mortalidad intrahospitalaria en resecciones pancreáticas mayores supone un $6,5 \%$, esto es el doble de mortalidad que tiene la cirugía de bypass coronario y 6 veces más que la cirugía de prótesis de cadera. Esta cifra aumenta notablemente en hospitales con bajo volumen. La mortalidad 
intrahospitalaria en pacientes mayores de 75 años a los que han sido sometidos a DPC en hospitales con alto volumen alcanza el $4,9 \%$, porcentaje que asciende hasta el $21 \%$ en hospitales con bajo volumen. Esta proporción es similar en otras resecciones pancreáticas (DPT y PD) (113).

En el año 2004, Dindo et al. publicaron en la prestigiosa revista Annals of Surgery una nueva escala de definición y estadificación de las complicaciones postoperatorias. Desde entonces, está escala ha sido validada a nivel internacional y es habitual su utilización en la práctica quirúrgica diaria y en la estandarización de comunicaciones científicas con el objetivo de facilitar la evaluación y comparación de resultados postquirúrgicos entre diferentes cirujanos, centros y técnicas quirúrgicas (114). El sistema de gradación de severidad de una complicación depende del tratamiento utilizado para subsanar dicha complicación y además este se relaciona de forma significativa con la complejidad de los procedimientos quirúrgicos y con la estancia hospitalaria postoperatoria. Las definiciones de los distintos grados de gravedad se reflejan en la tabla 4. En la tabla 5 se describen ejemplos clínicos de cada uno de estos niveles (114). 
Tabla 4: Definnición de los niveles de gravedad de las complicaciones quirúrgicas.

\begin{tabular}{|c|c|l|}
\hline \multicolumn{2}{|c|}{ Grado } & \multicolumn{1}{c|}{ Definición } \\
\hline \multicolumn{2}{|c|}{ I } & $\begin{array}{l}\text { Cualquier desviación del postoperatorio habitual. Se permiten: analgésicos, } \\
\text { antipiréticos, antieméticos y diuréticos. Se contempla también la infección } \\
\text { de herida quirúrgica (IHQ) explorada en la propia cama del enfermo. }\end{array}$ \\
\hline \multicolumn{2}{|c|}{ II } & $\begin{array}{l}\text { Otros tratamientos farmacológicos no contemplados en el grado I. Se } \\
\text { incluye la transfusión de hemoderivados y la nutrición parenteral. }\end{array}$ \\
\cline { 3 - 4 } III & IIIa & Intervención radiológica, endoscópica o quirúrgica sin anestesia general. \\
\hline \multirow{2}{*}{ IV } & IVa & $\begin{array}{l}\text { Fallo de un único órgano, con necesidad de manejo en unidades de cuidados } \\
\text { intensivos. }\end{array}$ \\
\cline { 2 - 3 } & IVb & $\begin{array}{l}\text { Fallo multiorgánico, con necesidad de manejo en unidades de cuidados } \\
\text { intensivos. }\end{array}$ \\
\hline & V & Muerte del paciente. \\
\hline
\end{tabular}

\begin{tabular}{|c|c|c|}
\hline \multicolumn{3}{|c|}{$\begin{array}{l}\text { Tabla 5: Ejemplos clínicos de cada uno de los niveles de gravedad de las complicaciones } \\
\text { quirúrgicas. }\end{array}$} \\
\hline \multicolumn{2}{|c|}{ Grado } & Ejemplo \\
\hline & [ & $\begin{array}{ll} & \text { Atelectasia que precisa fisioterapia. } \\
\text { - } & \text { Arritmia que puede corregirse con reposición electrolítica. } \\
\text { - } & \text { IHQ que se drena en la cama del enfermo. }\end{array}$ \\
\hline & $\mathbf{I}$ & $\begin{array}{ll} & \text { Arritmia que precisa tratamiento con antagonistas de receptores } \beta . \\
\text { - } & \text { Trombosis venosa profunda que requiere anticoagulantes. } \\
\text { - } & \text { Neumonía o infección urinaria tratada con antibióticos. }\end{array}$ \\
\hline \multirow[t]{2}{*}{ III } & IIIa & $\begin{array}{ll}\text { - } & \text { Bradiarritmia a la que hay que colocar marcapasos. } \\
\text { - } & \text { Estenosis ureteral que requiere la colocación de catéteres. } \\
\text { - } & \text { Colección intraabdominal que se drena percutáneamente mediante } \\
\text { radiología intervencionista. }\end{array}$ \\
\hline & IIIb & $\begin{array}{l}\text { - Reintervención, bien mediante laparotomía, toracotomía o apertura } \\
\text { de ventana pericárdica. }\end{array}$ \\
\hline \multirow[t]{2}{*}{ IV } & IVa & 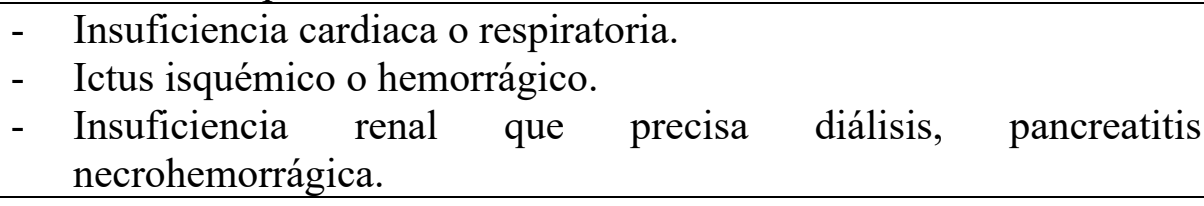 \\
\hline & $\mathbf{I V b}$ & - Combinación del fallo de 2 o más órganos. \\
\hline & & Muerte del paciente. \\
\hline
\end{tabular}




\subsection{2.- COMPLICACIONES ESPECÍFICAS}

\subsubsection{1.- FÍSTULA PANCREÁTICA POSTOPERATORIA}

Hasta el año 2005 existían veintiséis definiciones diferentes de FPPO, por lo que la comparación de los resultados entre diferentes centros resultaba muy complicada ya que no se tenía homogeneidad en la mencionada complicación. En ese año, un grupo formado por expertos en cirugía pancreática pertenecientes a diferentes países, se reunió para consensuar una definición objetiva de FPPO aceptada internacionalmente: cualquier débito o fluido que sale por un drenaje colocado de manera intraoperatoria con una medición de amilasa superior a 3 veces el límite superior de la normalidad del rango del laboratorio de referencia (115).

A pesar de las diferentes modificaciones técnicas y avances, la FPPO sigue siendo una de las complicaciones más relevantes con una incidencia que varía entre el 3\%-45\% de las cirugías pancreáticas en los centros de alto volumen (116).

La figura 8 resume las características principales del sistema de gradación de FPPO, según la última actualización del ISGPS. El grado de gravedad se define una vez que la evolución de la FPPO se haya completado en su totalidad y se pueda evaluar su efecto final sobre el resultado.

- Fístula bioquímica: Esta condición no conlleva por definición una repercusión clínica. Además, no supone una desviación del postoperatorio por lo que no afecta a la estancia postoperatoria. Si no se ha dejado un drenaje de manera intraoperatoria, el paciente no puede ser clasificado en este grado. Si el 
paciente muere durante el postoperatorio de manera repentina por infarto de miocardio, TEP, fallo renal que no se pueda explicar por la FPPO, la clasificación de la FPPO deberá seguir siendo fístula bioquímica (115).

- Fístula grado B: a este grado pertenecen aquellos pacientes que tienen repercusión clínica relevante asociado a un aumento de los niveles de amilasa medido en el débito del líquido de drenaje. La FPPO grado B requiere un cambio en el manejo postoperatorio normal después de una pancreatectomía o mantenimiento del drenaje durante más de 21 días o reposicionamiento de un drenaje guiado por pruebas de imagen debido a una colección postoperatoria $(115,117)$.

- Fístula grado $C$ : se define FPPO grado C cuando un paciente presenta inestabilidad clínica con fallo de órganos, reoperación o incluso muerte debido a esta causa. El fallo de órganos se define como la necesidad de reintubación, hemodiálisis y/o uso de drogas vasoactivas durante más de 24 horas por un fallo respiratorio, renal o cardiaco respectivamente (115). 
Figura 8: Diagrama de clasificación de grado de FPPO según ISGPS (115).

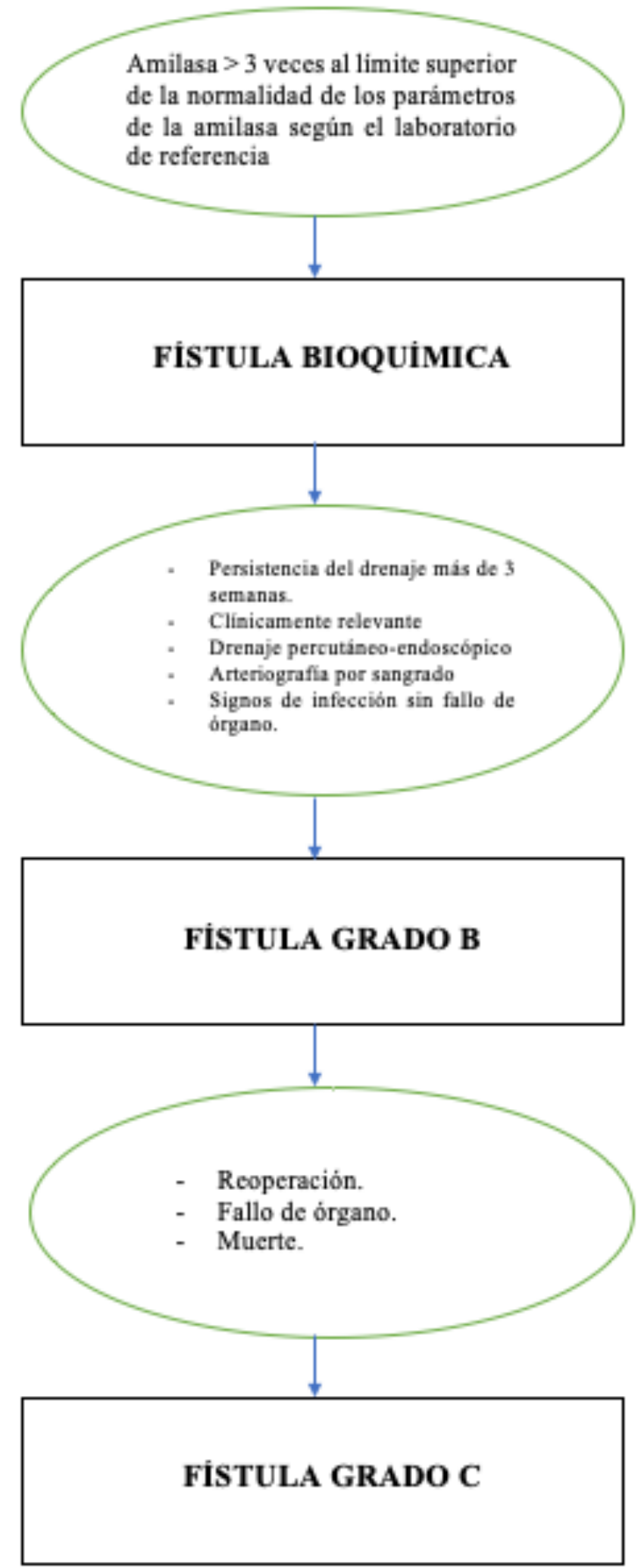


Los factores de riesgo asociados al aumento de la incidencia de aparición de FPPO son los siguientes:

\section{Tabla 6: Factores de riesgo asociados a la aparición de FPPO $(118,119)$.}

Textura blanda del páncreas.

Pérdida de sangre cuantiosa intraoperatoria.

Conducto de Wirsung fino.

Patología no pancreática.

Valor de albúmina preoperatoria disminuida.

El manejo de la FPPO depende de su sintomatología acompañante, la afectación localizada o sistémica, y si esta está correctamente drenada o bien se ha loculado. En un primer paso, se debe realizar un soporte nutricional e hidroelectrolítico adecuado. Muchos pacientes requieren nutrición parenteral total (NPT).

El octeotride, análogo de la somatostatina, tiene un papel algo controvertido en la reducción del débito de las fístulas de alto débito, pero no en el cierre de las mismas. En un metanálisis, de 7 estudios aleatorizados que incluía 297 pacientes, 102 pacientes desarrollaron FPPO. Las tasas de cierre de FPPO no fueron más altas en aquellos pacientes que fueron tratados con análogo de la somatostanina comparado con los controles (120).

En caso de que la FPPO sea clínicamente relevante (FPCR) (Grados B y C), se deben realizar acciones más agresivas para intentar controlar y paliar sus consecuencias. La endoscopia, utilizada para el drenaje de colecciones hacía la luz intestinal, tiene un 
papel limitado si en la fase de reconstrucción de una DPC se realiza en dos asas ya que el asa alimentaria y el asa biliar están separadas y no es posible llegar hasta la anastomosis pancreatoyeyunal por esta vía. Por otra parte, el drenaje percutáneo guiado por imagen, es una técnica eficaz y poco agresiva para el tratamiento de colecciones postoperatorias de FPPO encubierta.

\subsubsection{2.- GASTROPARESIA O RETRASO DEL VACIAMIENTO GÁSTRICO}

El RVG sin obstrucción mecánica puede ocurrir en el periodo postoperatorio después de cualquier cirugía supramesocólica, sirva de ejemplo la cirugía gástrica con vagotomía. El RVG es especialmente común después de cirugía pancreática y puede prolongar la estancia postoperatoria. Junto con la FPPO y la HPP, el RVG es una de las complicaciones más comunes tras cirugía pancreática cuya incidencia se sitúa entre el 19\%-57\% (121-123). El ISGPS también definió esta complicación con el objetivo, nuevamente, de homogeneizar los resultados postquirúrgicos.

El RVG se clasifica en A, B o C en función de la repercusión clínica (121). En la tabla 7 pueden observarse las características de cada uno de los grados. En ocasiones el RVG se relaciona con algún otro tipo de complicación, más frecuentemente en los grados B y C, como FPPO o absceso intraabdominal. En los grados B y particularmente C, es necesario descartar un diagnóstico alternativo que justifique el RVG utilizando pruebas más específicas con la TC o endoscopia. En la tabla 8 se resumen los parámetros característicos de cada grado del RVG. 
Introducción

Tabla 7: Definición por consenso de la ISGPS del RVG tras cirugía pancreática (121).

\begin{tabular}{|c|c|c|c|c|}
\hline Grado de RVG & SNG & $\begin{array}{l}\text { Imposibilidad } \\
\text { para tolerar } \\
\text { dieta sólida }\end{array}$ & Vómitos & $\begin{array}{c}\text { Uso de } \\
\text { procinéticos }\end{array}$ \\
\hline Grado $A$. & $\begin{array}{l}4-7 \text { días o } \\
\text { reinserción } \\
\text { después del } 3^{\circ} \\
\text { DPO. }\end{array}$ & 7 & +- & +- \\
\hline Grado B. & $\begin{array}{l}8-14 \text { días o } \\
\text { reinserción } \\
\text { después del } 7^{\circ} \\
\text { DPO. }\end{array}$ & 14 & + & + \\
\hline Grado $C$. & $\begin{array}{l}\text { 8-14 días o } \\
\text { reinserción } \\
\text { después del } 7^{\circ} \\
\text { DPO. }\end{array}$ & 21 & + & + \\
\hline
\end{tabular}




\section{Tabla 8: Parámetros característicos del RVG (121).}

\begin{tabular}{|c|c|c|c|}
\hline RVG & GRADO A & GRADO B & GRADO C \\
\hline Condición clínica & Buena & Poca incomodidad & Mala, incomodidad \\
\hline Comorbilidad & No & $\begin{array}{l}\text { Posible (FPPO, } \\
\text { absceso } \\
\text { intraabdominal) }\end{array}$ & $\begin{array}{l}\text { Posible (FPPO, } \\
\text { absceso } \\
\text { intraabdominal) }\end{array}$ \\
\hline $\begin{array}{l}\text { Tratamiento } \\
\text { específico }\end{array}$ & Posiblemente & $\mathrm{Si}$ & $\mathrm{Si}$ \\
\hline $\begin{array}{c}\text { Soporte } \\
\text { nutricional }\end{array}$ & Posiblemente & $\mathrm{Si}$ & $\mathrm{Si}$ \\
\hline $\begin{array}{l}\text { Evaluación } \\
\text { diagnóstica }\end{array}$ & No & Posiblemente & $\mathrm{Si}$ \\
\hline Intervencionismo & No & No & Posiblemente \\
\hline $\begin{array}{l}\text { Aumento de la } \\
\text { estancia } \\
\text { postoperatoria }\end{array}$ & Posiblemente & $\mathrm{Si}$ & $\mathrm{Si}$ \\
\hline $\begin{array}{l}\text { Retraso del } \\
\text { potencial } \\
\text { tratamiento } \\
\text { adyuvante }\end{array}$ & No & No & $\mathrm{Si}$ \\
\hline
\end{tabular}

Como ya se ha descrito previamente en este trabajo, existen algunas modificaciones en la técnica quirúrgica que pueden influir el RVG. Una de las controversias generadas en los últimos años es si la DPC con preservación pilórica aumenta o disminuye la posibilidad del RVG. Seis metanálisis recientemente publicados han puesto de manifiesto que la DPC con resección pilórica es la técnica requerida para mejorar mencionada complicación $(59,64-68)$. 


\subsubsection{3.- HEMORRAGIA POSTOPERATORIA POSTPANCREATECTOMÍA}

La mortalidad perioperatoria de la cirugía pancreática especialmente en centros de alto volumen se ha reducido sustancialmente en los últimos años. En un gran estudio alemán que analizó a 58003 pacientes a los que se realizó cirugía pancreática, la mortalidad intrahospitalaria fue $10,1 \%$ (124). Estos resultados han mejorado debido fundamentalmente gracias a los avances técnicos quirúrgicos, las mejoras en el manejo intensivo, la implementación de la radiología intervencionista y la mejoría en la selección del paciente. Sin embargo, la morbilidad postoperatoria aún se sitúa, dependiendo de las diferentes series, alrededor del 30-70\% (109-111).

La HPP tiene una incidencia del 1\% al 8\% después de una resección pancreática y contribuye en el 11\% al 18\% de la mortalidad global tras este tipo de intervención $(125,126)$. Esta puede originarse en el postoperatorio inmediato ( $<24$ horas), debido fundamentalmente a la falta de hemostasia intraoperatoria o a la coagulopatía no corregida, o en el postoperatorio tardío días después de la intervención o incluso semanas, la etiología en este caso suele corresponder a una FPPO no advertida que haya dañado los vasos adyacentes al área quirúrgica fomentando de esta manera el sangrado, una ulceración por el drenaje intraabdominal o incluso un pseudoaneurisma de la arteria gastroduodenal o hepática (127). La figura 9 muestra la localización del posible origen de la HPP. 
Figura 9: A. Localización del posible origen de la HPP derecha (1. Muñón de la arteria gastroduodenal. 2. Ramas tributarias de la vena porta y ramas de la AH. 3. Ramas de la VMS, incluyendo los vasos del proceso uncinado. 4. Ramas de la arteria mesentérica superior. 5. Línea de sección de la pancreatectomía y la anastomosis pancreatoyeyunal. 6. Lecho de la colecistectomía. 7. Línea de sutura de la duodenoyeyunostomía en caso de pancreatectomía con preservación pilórica. 8. Línea de sutura de la gastroyeyunostomía en caso de DPC clásica. 9. Retroperitoneo.)

B. Localización del posible origen de la HPP izquierda. (1. Sección pancreática. 2. Ramas tributarias de la arteria esplénica. 3. Hilio esplénico, en el caso de que se realice preservación esplénica. 4. Área de resección.). [Tomado de Wente et al. (128)].
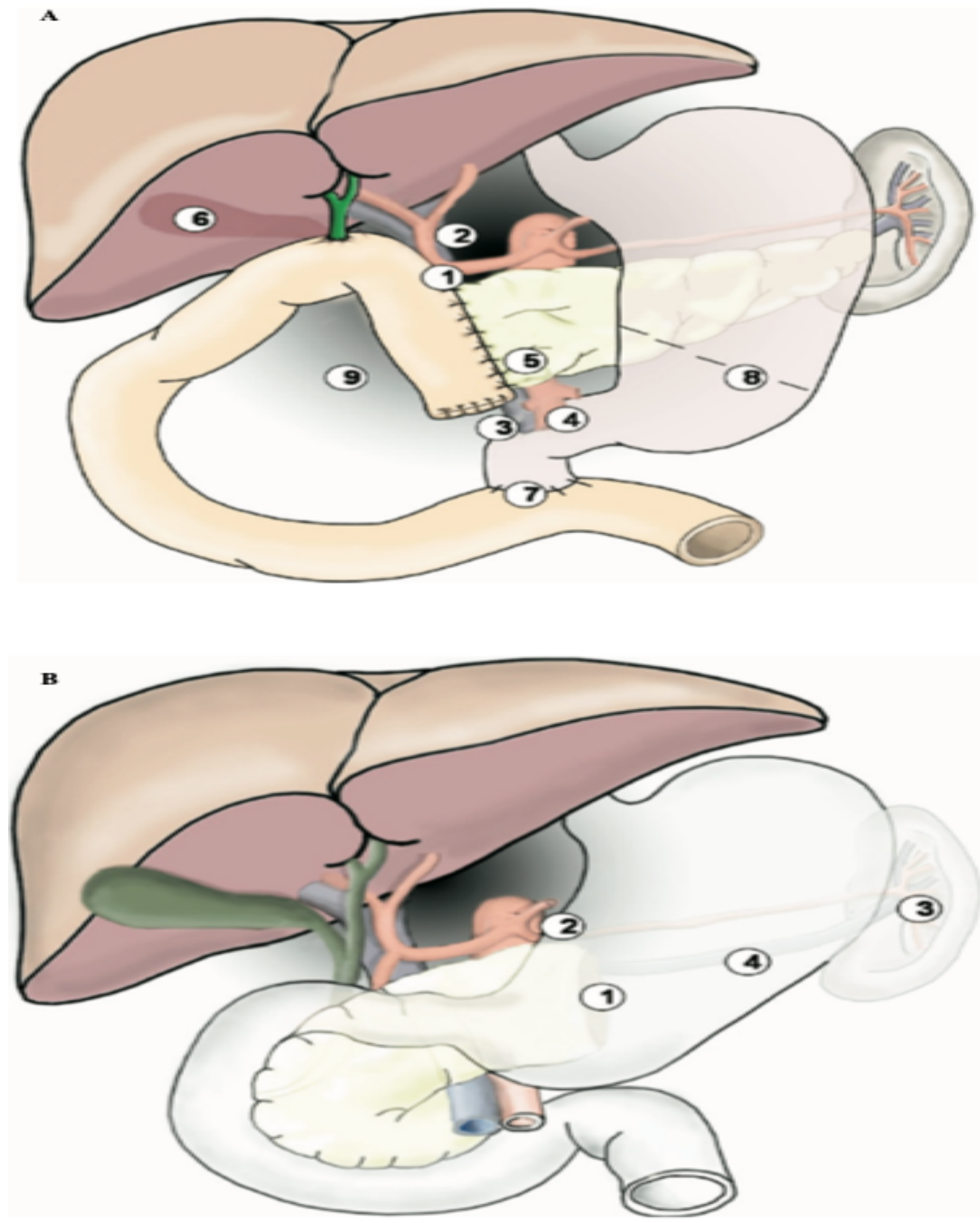
La definición que el ISGPS ha propuesto se divide en tres parámetros, tiempo transcurrido desde la intervención, la localización del sangrado y la gravedad de la hemorragia. Las características de estas definiciones pueden observarse en la tabla 9.

\section{Tabla 9: Definición propuesta por el ISGPS en HPP (128).}

\section{Tiempo transcurrido desde la intervención.}

- Hemorragia temprana ( $\leq 24$ horas desde la intervención).

- Hemorragia tardía ( $\geq 24$ horas desde la intervención).

\section{Localización.}

- Intraluminal (Sutura de la anastomosis, úlcera de estrés...).

- Extraluminal (Sangrado arterial, venoso, pseudoaneurisma...).

\section{Gravedad de la hemorragia.}

- Moderada:

- Pérdida de volumen sanguíneo bajo $<3 \mathrm{~g} / \mathrm{dL}$.

- Repercusión clínica moderada (Trasfusión de máximo 3 concentrados de hematíes).

- No necesidad de reintervención ni angiografía intervencionista.

- Severa:

- Pérdida de volumen sanguíneo alto $\geq 3 \mathrm{~g} / \mathrm{dL}$.

- Repercusión clínicamente relevante (Trasfusión sanguíneo mayor de 3 concentrados de hematíes).

- Necesidad de tratamiento invasivo (cirugía o angiografía).

Gramos por decilitro (g/dL)

La tabla 10 resume los diferentes factores que influyen en la HPP y establece un sistema de gradación clínico. Las tres diferentes categorías de la HPP (A, B y C) están definidos acorde al tiempo en el que se transcurre el sangrado, la localización y la gravedad del mismo (128). Como puede observarse en la mencionada tabla, el manejo de la HPP varía en función del grado de gravedad. En la HPP de grado A no se necesita normalmente ningún cambio terapéutico, ya que únicamente con una actitud de observación y expectante es suficiente. Por su parte, en la HPP de grado B, la trasfusión, 
los cuidados intermedios o incluso una actitud más agresiva, como es la relaparotomía para abordar de una manera temprana el sangrado, son necesarias. En cuanto a la HPP grado $\mathrm{C}$, técnicas como la angioembolización y relaparotomía son precisas para salvar la vida al enfermo.

\section{Tabla 10: Clasificación de la HPP de la ISGPS (128).}

\begin{tabular}{|c|c|c|c|c|}
\hline Grado. & $\begin{array}{c}\text { Tiempo, localización, } \\
\text { gravedad y } \\
\text { repercusión clínica. }\end{array}$ & $\begin{array}{l}\text { Condición } \\
\text { clínica. }\end{array}$ & $\begin{array}{l}\text { Consecuencia } \\
\text { diagnóstica. }\end{array}$ & $\begin{array}{c}\text { Consecuencia } \\
\text { terapéutica. }\end{array}$ \\
\hline $\mathbf{A}$ & $\begin{array}{l}\text { Temprana, intra o } \\
\text { extraluninal, } \\
\text { moderada. }\end{array}$ & Buena. & $\begin{array}{l}\text { Observación, } \\
\text { ecografía o } \\
\text { TC. }\end{array}$ & No. \\
\hline B & $\begin{array}{l}\text { Temprana o tardía, } \\
\text { intra o extraluminal, } \\
\text { moderada o severa. }\end{array}$ & $\begin{array}{c}\text { Normalmente } \\
\text { buena. }\end{array}$ & $\begin{array}{l}\text { Observación, } \\
\text { TC, } \\
\text { Angiografía, } \\
\text { endoscopia. }\end{array}$ & $\begin{array}{l}\text { Trasfusión, } \\
\text { cuidados } \\
\text { intermedios, } \\
\text { relaparotomía } \\
\text { para control } \\
\text { temprano de } \\
\text { HPP. }\end{array}$ \\
\hline $\mathbf{C}$ & $\begin{array}{c}\text { Tardía, intra o } \\
\text { extraluminal, severa. }\end{array}$ & $\begin{array}{l}\text { Amenaza la } \\
\text { vida. }\end{array}$ & $\begin{array}{c}\text { Angiografía, } \\
\text { endoscopia, } \\
\text { TC. }\end{array}$ & $\begin{array}{l}\text { Angiografía y } \\
\text { embolización, } \\
\text { relaparotomía. }\end{array}$ \\
\hline
\end{tabular}




\section{5.- ESCALAS PREDICTORAS DE RIESGO DE COMPLICACIÓN TRAS CIRUGÍA PANCREÁTICA}

La cirugía pancreática, como se ha descrito anteriormente, presenta una tasa de complicaciones nada desdeñable. A la luz de esta frecuencia notable de morbimortalidad postquirúrgica y dada la relevancia que tiene para el paciente, numerosos investigadores han analizado, desde hace años, qué variables clínicas preoperatorias suponen riesgo incrementado de aparición de complicaciones tras la resección pancreática. Así pues, existen diversos modelos predictores que confirmaron los siguientes factores de riesgo: datos de tipo demográfico (sexo masculino, edad avanzada), ponderal (sobrepeso y obesidad), comorbilidad basal (grado $\geq$ III de la escala de riesgo anestésico American Society of Anesthesiologists (ASA), índice de comorbilidad de Charlson, constantes prequirúrgicas (frecuencia cardiaca, tensión arterial sistólica), patología cardiovascular o respiratoria, alteración de la coagulación, tipo de tumor (cáncer de origen no pancreático, neoplasia diseminada), toma de medicamentos (corticoides), tamaño tumoral, parámetros analíticos prequirúrgicos (nivel de albúmina sérica, hemoglobina), intraoperatorios (textura del páncreas, diámetro del conducto de Wirsung, pérdida sanguínea, tipo de resección pancreática), etc. (129-137). Un resumen de estas características puede visualizarse en la tabla 11. 
Tabla 11: Variables preoperatorias asociadas con la aparición de complicaciones. Tras resección pancreática. [Obtenida de Rodriguez-Lopez et al (138)]

\begin{tabular}{|c|c|c|}
\hline & VARIABLES & $\begin{array}{l}\text { TIPO DE } \\
\text { COMPLICACION }\end{array}$ \\
\hline \multirow[t]{2}{*}{$\begin{array}{l}\text { Aoki } \\
\text { et al (129). }\end{array}$} & $\begin{array}{l}\text { Varón, edad avanzada, sobrepeso/obesidad, ASA } \\
\geq \mathrm{III} \text {, pérdida de peso }>10 \% \text {, dependencia para } \\
\text { actividades cotidianas, patología respiratoria o } \\
\text { cerebrovascular, tumor no pancreático, leucocitos } \\
>11000 / \mu 1 \text {, plaquetas }<80000 / \mu 1 \text {, International } \\
\text { Normalized Ratio (INR) }>1,25 \text {, albúmina }<2,5 \\
\mathrm{~g} / \mathrm{dL} \text {, creatinina }>2 \mathrm{mg} / \mathrm{dL} \text {, sodio }>146 \mathrm{mEq} / \mathrm{L} \text {, } \\
\text { PCR }>1,0 \mathrm{mg} / \mathrm{dL} \text {. }\end{array}$ & $\begin{array}{l}\text { Complicaciones } \\
\text { graves } \\
\text { (Clavien } \geq \mathrm{IV}) \text {. }\end{array}$ \\
\hline & $\begin{array}{l}\text { Varón, edad avanzada, sobrepeso/obesidad, ASA } \\
\geq \mathrm{III} \text {, dependencia para actividades cotidianas, } \\
\text { patología respiratoria, ascitis, infarto de } \\
\text { miocardio, enfermedad vascular periférica, tumor } \\
\text { no pancreático, hemoglobina }<7 \mathrm{~g} / \mathrm{dL} \text {, plaquetas } \\
<80000 / \mu 1, \mathrm{INR}>1,25 \text {, albumina }<2,5 \mathrm{~g} / \mathrm{dL} \text {, } \\
\text { creatinina }>2 \mathrm{mg} / \mathrm{dL} \text {, sodio }>146 \mathrm{mEq} / \mathrm{L}, \mathrm{PCR} \\
>1,0 \mathrm{mg} / \mathrm{dL} \text {. }\end{array}$ & $\begin{array}{l}\text { Fistula pancreática } \\
\text { (grado C). }\end{array}$ \\
\hline $\begin{array}{l}\text { Braga } \\
\text { et al }(130) \text {. }\end{array}$ & $\begin{array}{l}\text { ASA } \geq I I I, \text { Textura del páncreas, diámetro del } \\
\text { Wirsung, pérdida de sangre intraoperatoria. }\end{array}$ & $\begin{array}{l}\text { Complicaciones } \\
\text { graves (Clavien } \geq \mathrm{III}) \text {. }\end{array}$ \\
\hline $\begin{array}{l}\text { Callery et al } \\
\text { (131). }\end{array}$ & $\begin{array}{l}\text { Textura del páncreas, tipo patología, diámetro } \\
\text { Wirsung, pérdida sangre intraoperatoria. }\end{array}$ & $\begin{array}{l}\text { Fístula pancreática } \\
\text { grado B y C. }\end{array}$ \\
\hline $\begin{array}{l}\text { Gleeson } \\
\text { et al (132). }\end{array}$ & $\begin{array}{l}\text { HTA con tratamiento, edad }>62 \text { años, cirugía } \\
\text { cardiaca previa, coagulopatía, toma de corticoides, } \\
\text { albúmina }<3,5 \mathrm{~g} / \mathrm{dL} \text {, tumor diseminado, criterios } \\
\text { de Systemic Inflammatory Response Syndrome } \\
\text { (SIRS) presentes. }\end{array}$ & Mortalidad a 30 días. \\
\hline \multirow[t]{2}{*}{$\begin{array}{l}\text { Greenblatt } \\
\text { et al (133). }\end{array}$} & $\begin{array}{l}\text { Varón, edad avanzada, sobrepeso/obesidad, } \\
\text { dependencia para actividades cotidianas, patología } \\
\text { respiratoria, toma de corticoides, coagulopatía, } \\
\text { leucocitosis, creatinina }>1 \mathrm{mg} / \mathrm{dL} \text {, albúmina }<3,4 \\
\mathrm{~g} / \mathrm{dL} \text {. }\end{array}$ & $\begin{array}{l}\text { Complicaciones } \\
\text { graves } \\
\text { (sin emplear } \\
\text { clasificación de } \\
\text { Dindo-Clavien). }\end{array}$ \\
\hline & $\begin{array}{l}\text { Enfermedad pulmonar, HTA, radioterapia } \\
\text { preoperatoria, creatinina }>1,2 \mathrm{mg} / \mathrm{dL} \text {, } \\
\text { albúmina }<3,4 \mathrm{~g} / \mathrm{dL} \text {. }\end{array}$ & Mortalidad. \\
\hline $\begin{array}{l}\text { Kantor } \\
\text { et al }(134) \text {. }\end{array}$ & $\begin{array}{l}\text { Varón, sobrepeso/obesidad, } \\
\text { bilirrubina }<2 \mathrm{mg} / \mathrm{dL} \text {. }\end{array}$ & $\begin{array}{l}\text { FPCR (grados B y } \\
\text { C). }\end{array}$ \\
\hline $\begin{array}{l}\text { Venkat et al } \\
(135) \text {. }\end{array}$ & $\begin{array}{l}\text { Edad, sexo, tamaño tumoral, albúmina }<2,5 \mathrm{~g} / \mathrm{dL} \text {, } \\
\text { DPT, índice de comorbilidad de Charlson. }\end{array}$ & $\begin{array}{l}\text { Mortalidad a } 30 \text { y } 90 \\
\text { DPO. }\end{array}$ \\
\hline $\begin{array}{l}\text { Uzunoglu et } \\
\text { al (136). }\end{array}$ & $\begin{array}{l}\text { Albúmina }<3,5 \mathrm{~g} / \mathrm{dL} \text {, cirugía programada, tipo de } \\
\text { resección pancreática, tipo de patología, } \\
\text { frecuencia cardiaca lpm, tensión arterial sistólica } \\
\mathrm{mmHg} \text {, hemoglobina } \mathrm{g} / \mathrm{dL}, \mathrm{ASA}>\mathrm{III} \text {. }\end{array}$ & $\begin{array}{l}\text { Complicaciones } \\
\text { graves } \\
\text { (Clavien } \geq \text { IIIa). }\end{array}$ \\
\hline $\begin{array}{l}\text { Wiltberger } \\
\text { et al (137). }\end{array}$ & $\begin{array}{l}\text { Sobrepeso/obesidad, ASA } \geq \mathrm{III}, \text { comorbilidad } \\
\text { cardiovascular (incluida HTA), comorbilidad } \\
\text { respiratoria. }\end{array}$ & $\begin{array}{c}\text { Complicaciones } \\
\text { graves } \\
\text { (Clavien } \geq I I I b) .\end{array}$ \\
\hline
\end{tabular}

$\mu 1 \quad$ (microlitro), $\mathrm{g} / \mathrm{dL}$ (gramos/decilitro), $\mathrm{mg} / \mathrm{dL}$ (miligramos/decilitro), $\mathrm{mEq} / \mathrm{L}$ (miliequivalentes/litro), PCR (proteína C reactiva), $\mathrm{mmHg}$ (milímetros de mercurio), lpm (latidos por minuto). 


\subsection{1.- PREOPARTIVE PANCREATIC RESECTION (PREPARE) SCORE}

La escala PREPARE fue publicada en Annals of Surgery en el año 2014 por Uzunoglu et al. El artículo describe un estudio prospectivo multicéntrico cuyos participantes son el hospital universitario de Hamburgo, el hospital San Josef de Bochum, la universidad de Ruhr, el hospital universitario de Milán y el hospital universitario de Verona, entre el año 2005 y 2013 (136)

Como se ha relatado previamente, la morbimortalidad de la cirugía pancreática supone un gran problema tras este tipo de intervención (113). Por eso, el objetivo de este estudio fue la obtención de una escala de predicción prequirúrgica del riesgo de morbilidad. Presenta las siguientes características (136):

- Incluye 8 variables clínicas de fácil determinación: cifra de albúmina plasmática, realización de cirugía urgente o electiva, frecuencia cardiaca y presión arterial sistólica del paciente, técnica quirúrgica, patología estrictamente pancreática o de otra etiología, cifra de hemoglobina en sangre y grado en la escala de riesgo anestésico ASA. En función de los datos del paciente, se asigna o no una puntuación concreta por cada una de las variables [tabla 12]. De todas ellas, la que más puntos aporta es una cifra de albúmina inferior a 3,5 g/dL, siendo ésta un indicador de mala situación nutricional del paciente. Por otra parte, la inclusión de la variable "técnica quirúrgica" resulta de gran interés, puesto que los distintos procedimientos descritos en cirugía pancreática se asocian, también, con diferente tasa de complicaciones. Otras escalas publicadas, como la de Wiltberger et al. (137) que se expondrá a 
continuación, se puede emplear solamente en un tipo concreto de resección pancreática, como en este caso, la DPC.

- El registro de complicaciones se hizo empleando la mencionada clasificación de Dindo-Clavien (114), lo cual supone homogeneidad y exhaustividad en la definición de la severidad de las mismas, así como una fácil reproductibilidad por parte de otros investigadores. Se define la complicación mayor con un grado de Clavien $\geq$ IIIa.

- El cálculo de la puntuación del PREPARE se realiza antes de la intervención quirúrgica, empleando las mencionadas variables que son determinadas preoperatoriamente. Ello supone una importante diferencia respecto a otras escalas que emplean datos clínicos que, únicamente, pueden ser obtenidos tras completar la cirugía $(130,131)$. De acuerdo a los puntos obtenidos se establecen 3 grupos de riesgo: bajo, medio y alto [figura 10].

- Para su descripción, se empleó un número elevado de pacientes (703), reclutados en 5 centros europeos de alto nivel para cirugía pancreática. Posteriormente, 429 nuevos pacientes constituyeron la cohorte de validación interna de la propia escala, con recogida de datos prospectiva.

- Los datos estadísticos del test son muy favorables: estadístico $\mathrm{C}=0,709$, con fiabilidad diagnóstica del 75\% para predecir adecuadamente la presencia de complicaciones graves, según notificaron sus autores en el artículo. 


\begin{tabular}{|c|c|c|}
\hline VARIABLE & DATO & PUNTUACIÓN \\
\hline \multirow{2}{*}{$\begin{array}{c}\text { ALBÚMINA } \\
\text { PLASMÁTICA }\end{array}$} & $\geq 3,5 \mathrm{~g} / \mathrm{dL}$ & \\
\hline & $<3,5 \mathrm{~g} / \mathrm{dL}$ & 5 puntos \\
\hline \multirow{2}{*}{$\begin{array}{c}\text { CIRUGÍA } \\
\text { PROGRAMADA }\end{array}$} & $\mathrm{Si}$ & \\
\hline & No (urgente) & 4 puntos \\
\hline \multirow{2}{*}{$\begin{array}{c}\text { TÉCNICA } \\
\text { QUIRÚRGICA }\end{array}$} & Otras técnicas & \\
\hline & $\begin{array}{l}\text { DPC, resección de cabeza de páncreas } \\
\text { con preservación del duodeno }\end{array}$ & 2 puntos \\
\hline \multirow{2}{*}{$\begin{array}{l}\text { PATOLOGÍA DE } \\
\text { ORÍGEN } \\
\text { PANCREÁTICO }\end{array}$} & $\mathrm{Si}$ & \\
\hline & No & 2 puntos \\
\hline \multirow{2}{*}{$\begin{array}{l}\text { FRECUENCIA } \\
\text { CARDIACA }\end{array}$} & $50-801 \mathrm{lpm}$ & \\
\hline & $<50$ o $>801 \mathrm{lpm}$ & 2 puntos \\
\hline \multirow{2}{*}{$\begin{array}{c}\text { PRESIÓN } \\
\text { ARTERIAL } \\
\text { SISTÓLICA }\end{array}$} & $110-130 \mathrm{mmHg}$ & \\
\hline & $<110$ o $>130 \mathrm{mmHg}$ & 2 puntos \\
\hline \multirow{2}{*}{$\begin{array}{c}\text { HEMOGLOBINA } \\
\text { EN SANGRE }\end{array}$} & $11,5-17 \mathrm{~g} / \mathrm{dL}$ & \\
\hline & $<11,5$ o $>17 \mathrm{~g} / \mathrm{dL}$ & 1 punto \\
\hline \multirow{2}{*}{$\begin{array}{c}\text { RIESGO } \\
\text { ANESTÉSICO } \\
\text { ESCALA ASA }\end{array}$} & I y II & \\
\hline & $\geq \mathrm{III}$ & 1 punto \\
\hline & & \\
\hline
\end{tabular}


Figura 10: Categorías de riesgo de la escala PREPARE (bajo, medio y alto) según la puntuación obtenida y su estimación de la probabilidad de aparición de complicaciones graves (Clavien $\geq$ IIIa) [tomado de Uzunoglu et al. (136)].

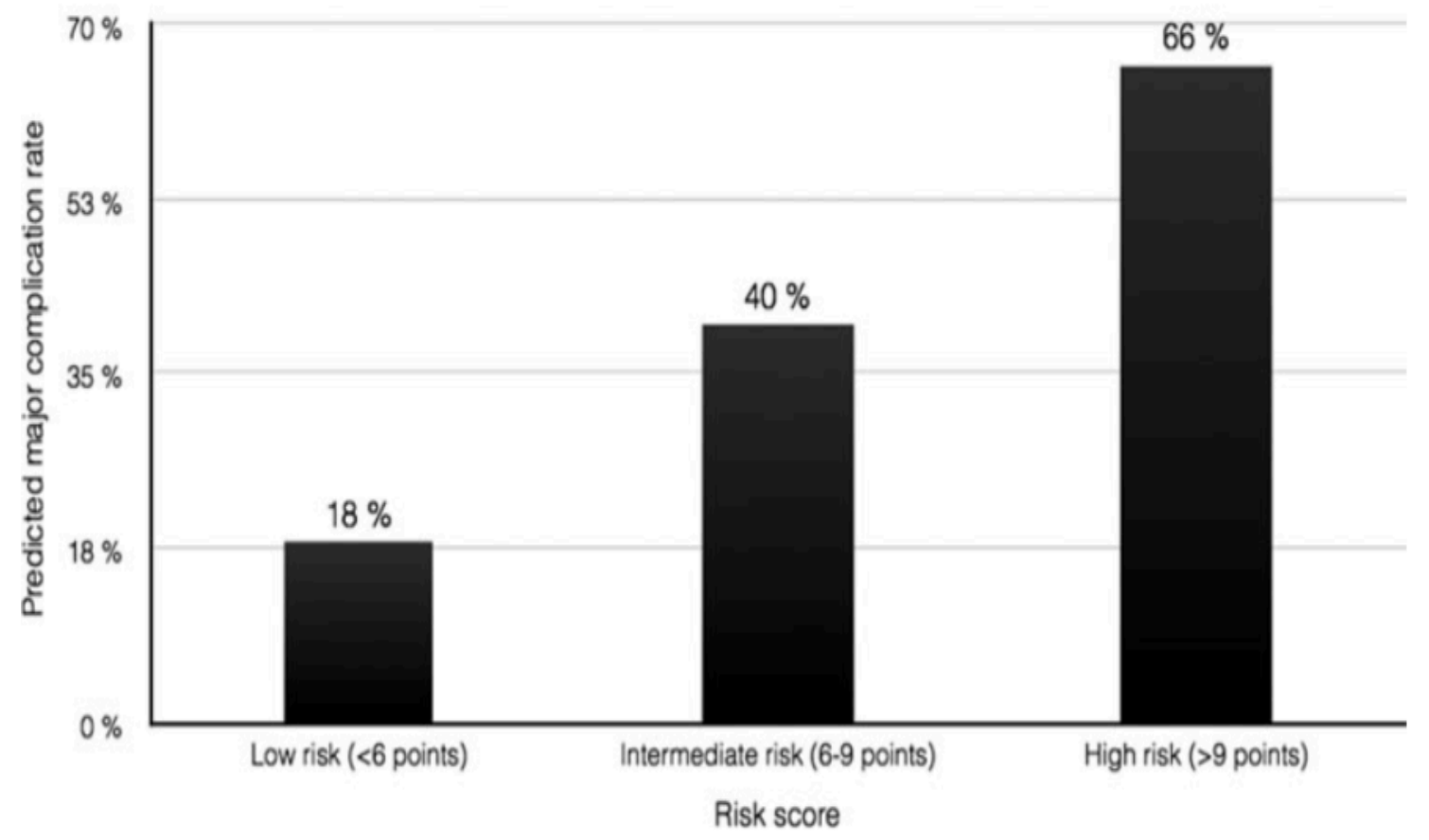

\subsection{2.- ESCALA DE WILTBERGER}

Este modelo fue descrito para predecir el riesgo de sufrir complicaciones graves en el postoperatorio de la DPC, de forma exclusiva y específica, a diferencia del PREPARE, que predice complicaciones en cualquier tipo de resección pancreática, como se ha indicado anteriormente. Las ventajas de la utilización de este modelo consisten en:

- Inclusión de 4 variables preoperatorias: la presencia de enfermedad cardiovascular, de enfermedad respiratoria o enfermedad pulmonar obstructiva crónica (EPOC), el grado en la escala de riesgo anestésico ASA, y el índice de masa corporal (IMC). En función de los datos del paciente, se asigna o no una puntuación concreta por cada una de las variables [tabla 13]. 
- Todos los parámetros que conforman la puntuación total son parámetros clínicos. Esto aporta sencillez en el momento de recoger las variables necesarias.

- Empleo, también en este índice, de la mencionada clasificación de DindoClavien (114) para el registro de complicaciones. Sin embargo, a diferencia de la escala PREPARE, la escala de Wiltberger define las complicaciones mayores con un grado de Clavien $\geq$ IIIb.

- Así mismo, en la escala de Wiltberger se obtienen las variables antes de realizar la cirugía. De acuerdo a la puntuación obtenida se asigna una categorización de riesgo: bajo, medio y alto [Figura 11].

- Fueron reclutados un total de 405 pacientes que iban a ser sometidos a DPC y el análisis de los mismos fue retrospectivo.

- Los datos estadísticos del test son: estadístico $\mathrm{C}=0,83$, por lo que según concluyen los autores se trata de un buen test capaz de predecir la aparición de complicaciones Clavien graves ( $\geq$ IIIb).

Finalmente, En la tabla 14 se puede observar esquemáticamente las diferencias más relevantes en la comparación de ambos índices predictores de complicaciones. 


\begin{tabular}{|c|c|c|}
\hline VARIABLE & DATO & PUNTUACIÓN \\
\hline \multirow[t]{3}{*}{ IMC } & $<25 \mathrm{Kg} / \mathrm{m}^{2}$ & \\
\hline & $\geq 25$ a $30 \mathrm{Kg} / \mathrm{m}^{2}$ & 1 punto \\
\hline & $\geq 30 \mathrm{Kg} / \mathrm{m}^{2}$ & 4 puntos \\
\hline \multirow[t]{2}{*}{ ASA } & $\leq \mathrm{II}$ & \\
\hline & $\geq \mathrm{III}$ & 2 puntos \\
\hline \multirow{2}{*}{$\begin{array}{c}\text { ENFERMEDAD } \\
\text { CARDIOVASCULAR }\end{array}$} & No & \\
\hline & $\mathrm{Si}$ & 6 puntos \\
\hline \multirow{2}{*}{$\begin{array}{c}\text { ENFERMEDAD } \\
\text { RESPIRATORIA/EPOC }\end{array}$} & No & \\
\hline & $\mathrm{Si}$ & 4 puntos \\
\hline
\end{tabular}

Kilogramos por metro cuadrado $\left(\mathrm{Kg} / \mathrm{m}^{2}\right)$.

Figura 11: Categorías de riesgo de la escala Wiltberger (bajo, medio y alto) según la puntuación obtenida y su estimación de la probabilidad de aparición de complicaciones graves (Clavien $\geq \mathrm{IIIb}$ ) [tomado de Wiltberger et al. (137)].

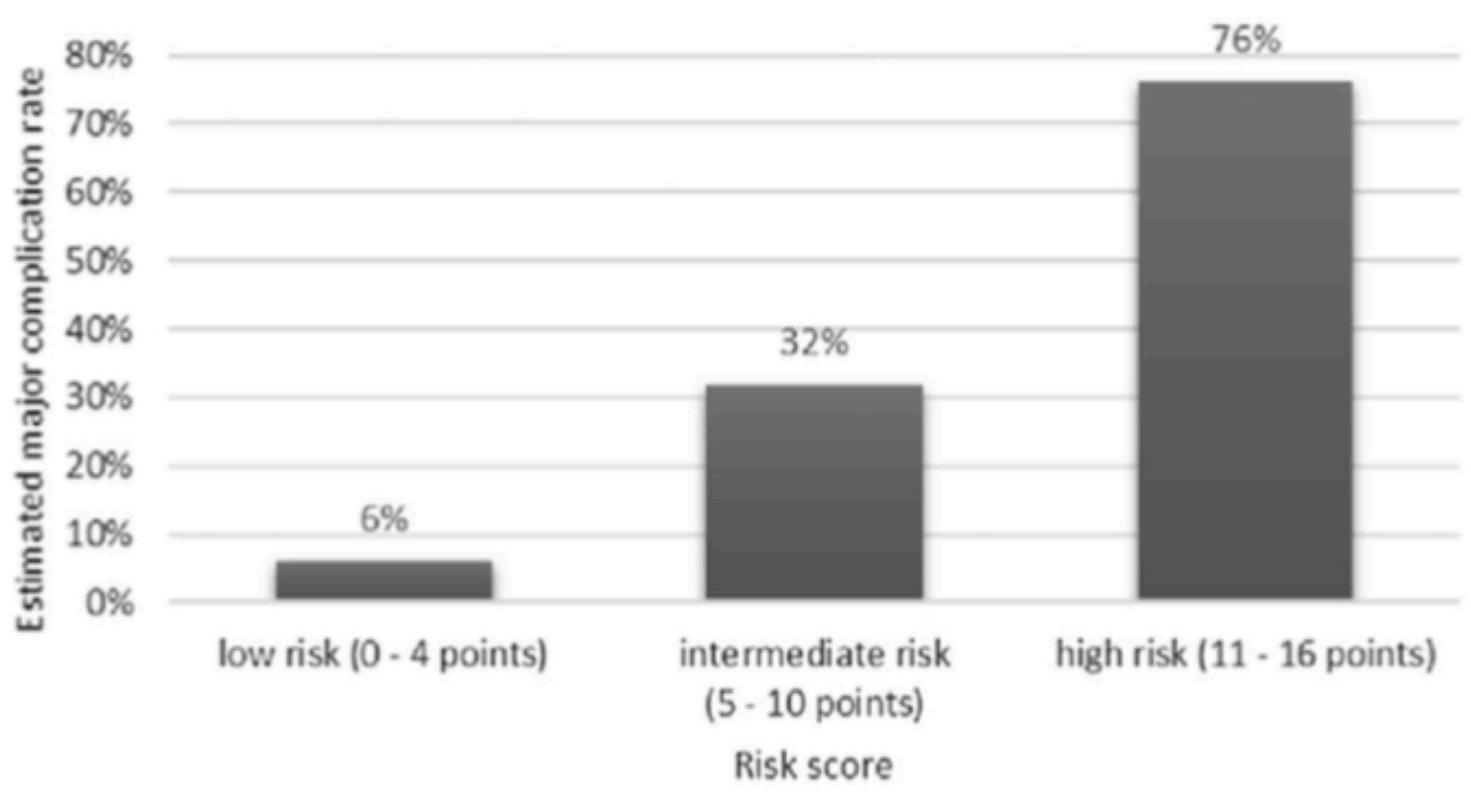




\begin{tabular}{|c|c|c|}
\hline VARIABLE & PREPARE & WILTBERGER \\
\hline Escala ASA & $\mathrm{X}$ & $\mathrm{X}$ \\
\hline $\begin{array}{l}\text { EPOC/ Enfermedad } \\
\text { respiratoria }\end{array}$ & & $\mathrm{X}$ \\
\hline Presión arterial sistólica & $\mathrm{X}$ & \\
\hline Hemoglobina & $\mathrm{X}$ & \\
\hline Albúmina & $\mathrm{X}$ & \\
\hline Cirugía no pancreática & $\mathrm{X}$ & \\
\hline Cirugía electiva & $\mathrm{X}$ & \\
\hline Frecuencia cardiaca & $X$ & \\
\hline IMC & & $X$ \\
\hline $\begin{array}{l}\text { Enfermedad } \\
\text { cardiovascular }\end{array}$ & & $\mathrm{X}$ \\
\hline $\begin{array}{l}\text { Tipo de técnica } \\
\text { quirúrgica }\end{array}$ & $\begin{array}{c}\text { Todas las resecciones } \\
\text { pancreáticas }\end{array}$ & DPC \\
\hline Complicación grave & Clavien $\geq$ IIIa & Clavien $\geq$ IIIb \\
\hline $\begin{array}{l}\text { Tipo de estudio usado } \\
\text { para definir la escala }\end{array}$ & $\begin{array}{c}\text { Prospectivo. } \\
\text { Multicéntrico. } \\
\text { Tamaño muestral alto. } \\
\text { Subgupo de validación } \\
\text { interna. }\end{array}$ & $\begin{array}{c}\text { Retrospectivo. } \\
\text { Unicéntrico. } \\
\text { Tamaño muestral menor. } \\
\text { Sin grupo de validación } \\
\text { interna. }\end{array}$ \\
\hline
\end{tabular}




\section{6.- JUSTIFICACIÓN}

Las resecciones pancreáticas mayores suponen una elevada morbimortalidad postoperatoria, todo esto conlleva una prolongación de la estancia postoperatoria y un mayor consumo de recursos. Las escalas predictoras de morbilidad no son usadas de manera sistematica en este tipo de cirugías.

Desde que, en 2014, fue publicado el trabajo de Uzunoglu et al. (136), solo cuatro nuevos estudios hasta la fecha han analizado la escala PREPARE sobre pacientes sometidos a cirugía pancreática procedentes de centros distintos de aquellos en los que se describió. El primero de ellos fue publicado en febrero de 2016 y es una serie retrospectiva turca de 122 individuos operados entre 2010 y 2015 (139). Estos autores concluyeron que la puntuación propuesta por el grupo de Hamburgo es la escala más útil y sencilla de todos las existentes, aunque su fiabilidad no es elevada ( $70 \%$, según sus resultados).

El segundo artículo, realizado por nuestro grupo, incluye los resultados preliminares de una serie de 18 pacientes analizados para realizar una validación externa prospectiva (140). Finalmente, dos años más tarde se publicó un segundo artículo después de haber aumentado el tamaño muestral con 50 pacientes (138). Aunque el análisis estadístico fue muy limitado, como consecuencia del bajo tamaño muestral, nuestros resultados mostraron que sí existían diferencias estadísticamente significativas para el porcentaje de complicaciones graves entre las 3 categorías 
Por último, en el año 2018, Samim et al. (141) publicaron un estudio cuyo objetivo fue comparar la predicción de las escalas pronósticas de complicación en cirugía hepatobiliopancreática con la opinión del cirujano. Entre las escalas que se evaluaron, la escala PREPARE estaba incluida en los 193 pacientes que fueron sometidos a cirugía pancreática.

Tras una exhaustiva búsqueda bibliográfica, no se ha encontrado ninguna otra publicación que realice una validación externa del escala de Uzunoglu et al. (136). De este modo, la posibilidad de re-evaluar esta escala tras reclutar nuevos pacientes en nuestra serie, es de un altísimo interés científico y quirúrgico, ya que ningún otro grupo de investigadores en España lo ha realizado.

En cuanto a la escala de Wiltberger usada únicamente para predecir el riesgo de complicaciones en la DPC, desde su publicación en el año 2016, tampoco existe ningún trabajo en el que se haya validado esta escala. El artículo original de Wiltberger et al. (137) reclutó y analizó pacientes de manera retrospectiva.

Por último, no se ha publicado ningún trabajo que compare ambas escalas, no pudiendo determinar así cuál de ellas tiene mayor fiabilidad pronóstica. 
Francisco Javier Tejero Pintor 


\section{HIPÓTESIS Y OBJETIVOS}


Francisco Javier Tejero Pintor 


\section{HIPÓTESIS Y OBJETIVOS}

\section{1.- HIPÓTESIS}

Las escalas PREPARE y Wiltberger pueden predecir el riesgo de complicaciones postoperatorias de pacientes sometidos a cualquier resección pancreática $\mathrm{y}$ duodenopancreatectomía cefálica, respectivamente. Ambas permiten categorizar a los pacientes en tres grupos de riesgo. Empleándolas de manera preoperatoria en el subgrupo de pacientes sometidos a duodenopancreatectomía cefálica (SDPC), una de ellas se comportará como predictor más fiable.

\section{2.- OBJETIVOS}

1.- Describir en una muestra de pacientes sometidos a cirugía pancreática en nuestro centro, notificando las complicaciones postoperatorias sufridas por los mismos y clasificarlas empleando una escala validada de severidad (clasificación de DindoClavien) tanto en la serie global como en el subgrupo de pacientes sometidos a duodenopancreatectomía cefálica.

2.- Calcular la puntuación en la escala PREPARE para cada paciente en la serie global, clasificar a los pacientes en las tres categorías de riesgo y determinar la proporción de complicaciones graves que finalmente presentaron los pacientes incluidos en cada categoría de riesgo prequirúrgico. 
3.-Calcular la escala de PREPARE en el subgrupo de pacientes sometidos a duodenopancreatectomía cefálica, clasificar a los pacientes en las tres categorías de riesgo y determinar la proporción de complicaciones graves que finalmente presentaron los pacientes incluidos en cada categoría de riesgo prequirúrgico.

4.- Calcular la escala de Wiltberger en el subgrupo de pacientes sometidos a duodenopancreatectomía cefálica, clasificar a los pacientes en las tres categorías de riesgo y determinar la proporción de complicaciones graves que finalmente presentaron los pacientes incluidos en cada categoría de riesgo prequirúrgico.

5.- Comprobar la validez de ambas escalas con pruebas estadísticas avanzadas y, además, en el subgrupo de pacientes sometidos a duodenopancreatectomía cefálica establecer cuál de ellas obtiene mayor fiabilidad.

6.- Comprobar si la escala PREPARE en la serie global y subgrupo de pacientes sometidos a duodenopancreatectomía cefálica y la escala Wiltberger en el subgrupo de pacientes sometidos a duodenopancreatectomía cefálica se asocia a otras variables de morbimortalidad postquirúrgica. 


\section{PACIENTES Y MÉTODOS}


Francisco Javier Tejero Pintor 


\section{PACIENTES Y MÉTODOS}

\section{1.- TIPO DE ESTUDIO}

El diseño del estudio fue observacional de tipo cohortes a partir de una base de datos prospectiva.

\section{2.- POBLACIÓN DE ESTUDIO}

La población a estudio fueron los pacientes con una patología susceptible de tratamiento quirúrgico mediante resección pancreática. Todos los enfermos fueron intervenidos por cirujanos expertos de la Unidad de Cirugía hepatobiliopancreática del Hospital Universitario Río Hortega (HURH) de Valladolid. El periodo de inclusión en el estudio comprende desde enero de 2015 hasta mayo de 2019.

Se incluyeron datos epidemiológicos, antropométricos, analíticos, operatorios, relativos a los antecedentes médico-quirúrgicos, al estudio preoperatorio, y a todas las posibles complicaciones postoperatorias durante los 30 primeros días del periodo postoperatorio o hasta el momento del alta hospitalaria en caso de los ingresos superiores a 30 días. Se utilizaron números en clave para la identificación de los pacientes, teniéndose en cuenta las normas de confidencialidad a ese respecto. 


\subsection{1.- CRITERIOS DE INCLUSIÓN}

Los criterios empleados para la inclusión de enfermos en la muestra que se analizó en este estudio fueron los siguientes:

- Ser mayor de 18 años.

- Firma de consentimiento informado para la participación en el estudio y para realizar la cirugía.

- Resección pancreática mayor (DPT, DPC, PD) realizada por la unidad de cirugía Hepatobiliopancreática del HURH de Valladolid.

Todos los enfermos candidatos a resección pancreática, son valorados en la sesión clínica de tumores hepatobiliopancreáticos. En ella forman parte cirujanos generales de la unidad mencionada, radiólogos y oncólogos. De esta manera, se garantiza un manejo homogéneo y multidisciplinar del paciente susceptible a tratamiento quirúrgico, y acorde a esto, la decisión e indicación es consensuada.

\subsection{2.- CRITERIOS DE EXCLUSIÓN}

Se excluyeron del presente estudio todos aquellos pacientes que presentaron uno o más de los siguientes criterios, a saber:

- Criterios de irresecabilidad tumoral durante el acto quirúrgico, y por este motivo no pudo completarse la resección pancreática.

- Resecciones pancreáticas en contexto de otro procedimiento quirúrgico (oncológico o no oncológico).

- Enucleaciones. 
- Resecciones pancreáticas no realizadas por la unidad de cirugía hepatobiliopancreática.

\subsection{3.- TAMAÑO MUESTRAL}

La muestra estuvo formada por todos aquellos individuos de la población a estudio que quedaron seleccionados tras aplicar los criterios de inclusión y exclusión. Se reclutaron consecutivamente todos los casos intervenidos durante el periodo de inclusión, teniendo en cuenta los mencionados criterios. Se empleó la base de datos prospectiva con los pacientes intervenidos por la unidad de cirugía hepatobiliopancreática del HURH de Valladolid durante el mencionado periodo del trabajo.

Con el objetivo de estructurar de forma concisa la exposición, se definió, por una parte, la serie global (SG) de la muestra, que incluye a todos los pacientes reclutados con cualquier tipo de resección pancreática, sobre los cuales se analizó la escala PREPARE únicamente, dado que la escala Wiltberger solo está definida para enfermos sometidos a DPC.

En segundo lugar, se seleccionaron los individuos de la muestra sometidos a la técnica de DPC, constituyendo el llamado SDPC. En este subgrupo, se volvieron a registrar las variables demográficas y aquellas que forman parte de la escala PREPARE y Wiltberger. 


\section{3.- CIRCUITO ASISTENCIAL DEL PACIENTE}

\subsection{1.- PREOPERATORIO}

El paciente acude a consulta de Cirugía General y Aparato Digestivo, a la unidad de cirugía hepatobiliopancreática o es referido a la unidad estando ingresado en la planta de Aparato Digestivo, con el diagnóstico de presunción de tumor de páncreas, tumor ampular, tumor de duodeno o colangiocarcinoma distal. En ocasiones este diagnóstico esta confirmado con biopsias. En caso de que exista una estenosis tumoral de la vía biliar principal o la clínica con la que el paciente comenzó su proceso tumoral fuera de sepsis de origen biliar, queda a criterio del endoscopista la colocación de una prótesis biliar en el mismo procedimiento diagnóstico.

Se realizan las siguientes pruebas diagnósticas para el estudio de extensión:

- TC de tórax, abdomen y pelvis.

- Si en la TC existe sospecha de metástasis hepática, se puede completar el estudio de extensión con una resonancia magnética hepática o con una ecografía con potenciadores.

- La tomografía por emisión de positrones se utiliza para aquellos casos en los que existen dudas de enfermedad a distancia no corroboradas por las anteriores pruebas.

En caso de indicarse la cirugía, se le explica al paciente el tipo de técnica y resección pancreática necesaria para su correcto tratamiento, y si se va a realizar por abordaje laparotómico o laparoscópico. Por lo tanto, se le informa de los riesgos 
quirúrgicos asociados a la cirugía pancreática en general, y de la técnica que se va a emplear en particular. Además se advierte del riesgo que existe durante la cirugía de modificar la técnica que se ha había planeado hacia otra distinta en función de los hallazgos intraoperatorios. Una vez informado, al paciente se le solicita por escrito el consentimiento para realizar la intervención quirúrgica.

Previamente a la cirugía, el paciente acude a la consulta de preanestesia. El anestesista recopila la información clínica del enfermo, revisando y evaluando todos los datos (situación clínica, pruebas complementarias realizadas). Se determina el grado de riesgo anestésico según la escala ASA y se valora cuidadosamente la vía aérea, estableciendo en caso necesario la activación del protocolo de vía aérea difícil, según los criterios anestésicos vigentes. De igual modo, se considera, según criterio clínico, la solicitud de fisioterapia respiratoria o cualquier otra prueba o procedimiento, a la vez que se determina el ajuste de medicación del enfermo previo a la intervención. Por último, se informa al paciente de la técnica anestésica y de los riesgos inherentes a la misma, y de la necesidad de estancia en la Unidad de reanimación, al menos las primeras 24-48 horas de postoperatorio. Una vez informado, se le solicita por escrito el consentimiento para la anestesia.

En ocasiones, queda a criterio del cirujano, suministrar soporte nutricional con batidos hiperproteicos hasta la realización de la cirugía. Otras veces, el paciente puede ser derivado al servicio de endocrinología y nutrición para que se realice un estudio nutricional y sea tratado antes de la intervención quirúrgica. 


\subsection{2.- PREOPERATORIO INMEDIATO}

El paciente ingresa en el HURH de Valladolid el día previo a la cirugía en horario de tarde a partir de las 17:00. Se realiza una exploración física sistemática y se revisa la historia clínica para comprobar que no exista ninguna enfermedad que contraindique la cirugía. Se toman las constantes (tensión arterial, frecuencia cardiaca, saturación de oxígeno) y medidas antropométricas (peso y talla). Se pauta su medicación habitual, heparina de bajo peso molecular a dosis profilácticas (una dosis de 3500 UI de bemiparina por vía subcutánea a las 20:00), medias de compresión elásticas en las extremidades inferiores y una benzodiacepina de acción corta o intermedia (lorazepam $1 \mathrm{mg}$ vía oral) antes de dormir.

El mismo día de la cirugía, a las 7:30 de la mañana, se realiza una analítica sanguínea en la que se solicita hemograma, bioquímica, coagulación, PCR, albúmina, prealbúmina, antígeno carcinoembrionario (CEA), antígeno carbohidrato 19-9 (CA 19-9) y antígeno carbohidrato 125 (CA 125).

Se administra Piperacilina-tazobactam 4-0,5 g por vía intravenosa durante la inducción anestésica en quirófano. En alérgicos a betalactámicos, se sustituye por ciprofloxacino $400 \mathrm{mg}$ por vía intravenosa.

\subsection{3.- TÉCNICA QUIRÚRGICA}

El paciente se coloca en decúbito supino con los brazos en cruz, además de sujeciones en las plantas de los pies y protecciones adecuadas en la región de los hombros 
para poder soportar la posición de Trendelemburg y antitrendelemburg (únicamente para el abordaje laparoscópico), minimizando así el riesgo de lesión del plexo braquial. En abordaje laparotómico, en algunas ocasiones, se utiliza un rodillo inflable entre la mesa y el dorso con el objetivo de abrir el ángulo de ambos arcos costales y la apófisis xifoides, mejorando así la exposición del campo quirúrgico. Se colocan en ambas extremidades inferiores compresión neumática intermitente. Desde el punto de vista anestésico, se realiza anestesia general balanceada combinada con epidural, con intubación orotraqueal (empleando fibroscopio en los enfermos catalogados como "vía aérea difícil) y se canulan vías (dos vías venosas periféricas, una de ellas de grueso calibre así como, preferentemente, una vía venosa yugular derecha y vía arterial radial izquierda). La monitorización de constantes vitales se realiza según criterio del anestesista. Se coloca sonda vesical con termómetro incorporado a todos los pacientes, extremando las medidas de asepsia. El procedimiento quirúrgico es llevado a cabo por dos cirujanos adjuntos de la unidad de cirugía hepatobiliopancreática y un médico interno residente del servicio de cirugía del HURH. Los cirujanos adjuntos que han formado parte en la realización de resecciones pancreáticas fueron ocho. La técnica quirúrgica realizada dependerá de la localización de afectación del tumor (como ya se ha explicado previamente). Al finalizar la cirugía, el cirujano responsable tiene la opción de decidir colocar un drenaje previo al cierre del enfermo. En caso de prolongación del tiempo quirúrgico se administra una nueva dosis de antibiótico. Queda a criterio del anestesista realizar la extubación en quirófano o bien trasladarlo a la unidad de Reanimación postquirúrgica intubado. 


\subsection{4.- PROTOCOLO POSTOPERATORIO}

Los pacientes pasan desde el quirófano a la unidad de Reanimación postquirúrgica para el control hemodinámico, respiratorio y balance hidroelectrolítico. A las 8:00 del primer DPO, se le realiza una analítica con hemograma, bioquímica, coagulación, PCR, procalcitonina, lactato arterial. Normalmente, queda a criterio del anestesista encargado del cuidado postoperatorio mantener al enfermo 24 horas más en unidad de Reanimación postquirúrgica. A las 48 horas postoperatorias, se le realiza una nueva analítica con los mismos parámetros analíticos del primer DPO. Cuando el paciente está estabilizado, a criterio del anestesista, el paciente es trasladado a la planta. En el quinto DPO, se realiza una nueva analítica con los mismos parámetros solicitados a las 24 y 48 horas (excepto el lactato que en este caso es venoso, debido a que el paciente ya no es portador de catéter arterial). Además, si el paciente tiene drenaje intraabdominal, se realiza un estudio analítico del débito del drenaje con amilasa.

Los cuidados requeridos de manera habitual durante el postoperatorio inmediato son los siguientes:

- Profilaxis de la úlcera de estrés: se administra un inhibidor de la bomba de protones a las dosis habituales por vía intravenosa hasta la tolerancia oral.

- Profilaxis de la enfermedad tromboembólica: se mantiene el vendaje compresivo en extremidades inferiores hasta el alta hospitalaria. Se continua con la administración de heparina de bajo peso molecular a dosis adecuadas durante todo el ingreso y cuando el paciente es dado de alta, durante un mes postoperatorio. Se moviliza al sillón desde el $2^{\circ}$ día postoperatorio y se 
estimula la deambulación precoz, dependiendo de la situación clínica del enfermo.

- Retirada de la SNG a la mayor brevedad posible (en el caso en el que haya sido necesaria su colocación) y retirada de la sonda vesical a las 24 horas, siempre que la situación clínica lo permita.

- Control del débito del drenaje, en caso de que se haya colocado. Retirada del mismos a la mayor brevedad posible, siempre que la situación clínica lo permita.

- Retirada de material de sutura entre los días 10-12 del postoperatorio (se puede realizar de forma ambulatoria en su Centro de Salud).

Ante el eventual diagnóstico de complicaciones postoperatorias, ya sean médicas o quirúrgicas, se actúa convenientemente, con el tratamiento más adecuado, incluyendo reintervención quirúrgica y/o un nuevo traslado a la Unidad de Reanimación postquirúrgica.

El manejo postoperatorio se lleva a cabo en función del criterio del cirujano responsable. Habitualmente, la dieta oral se introduce el segundo o tercer DPO y si la evolución es favorable, el paciente es dado de alta entre el séptimo y el décimo DPO.

\section{4.- VARIABLES DESCRITAS}

\subsection{1.- VARIABLES DEMOGRÁFICAS}

Las variables demográficas que se analizaron fueron las siguientes: 
- Sexo: variable cualitativa dicotómica (hombre o mujer).

- Edad: variable cuantitativa, medida en años en el momento de la intervención

\subsection{2.- VARIABLES PREOPERATORIAS}

- Antecedentes médicos personales:

- HTA: variable cualitativa dicotómica (sí/no).

○ Diabetes mellitus: variable cualitativa dicotómica (sí/no).

- Dislipemia: variable cualitativa dicotómica (sí/no).

- Tabaquismo: variable cualitativa dicotómica (sí/no).

- Consumo de alcohol: variable cualitativa dicotómica (sí/no). Establecido como al menos 1 bebida estándar o 10 gramos de alcohol a la semana, equivalente a una caña de cerveza 25 centilitros o un vaso de vino 10 centilitros.

- Prótesis biliar preoperatoria: Variable cualitativa dicotómica (si/no). A criterio del endoscopista en función de grado de obstrucción de la vía biliar principal en tumores adyacentes a dicha estructura anatómica

Como se ha descrito anteriormente, con el objetivo de estructurar de forma concisa este epígrafe, se ha definido, por una parte, la SG de la muestra, que incluye a todos los pacientes reclutados con cualquier tipo de resección pancreática, sobre los cuales se analizó la escala PREPARE únicamente, dado que la escala Wiltberger sólo está definido para enfermos sometidos a DPC.

- Variables incluidas en la escala PREPARE (136): 
- Cifra de albúmina: variable cuantitativa, medida en g/dL, y determinada mediante analítica de sangre, obteniendo la muestra inmediatamente antes del traslado del paciente a quirófano. A efectos del cálculo de la escala de PREPARE, la cifra de albúmina se ha definido como variable dicotómica $(<3,5 \mathrm{~g} / \mathrm{dL}$ o $\geq 3,5 \mathrm{~g} / \mathrm{dL})$.

○ Tipo de procedimiento: variable dicotómica (urgente o programado).

- Técnica quirúrgica: definida como variable dicotómica (DPC o PD y DPT, conjuntamente).

○ Patología de origen pancreático: variable dicotómica (sí o no).

- Frecuencia cardiaca: variable cuantitativa, medida en latidos por minuto (lpm), en el momento del ingreso en planta del paciente. A efectos del cálculo de la escala de PREPARE, la cifra de frecuencia cardiaca se ha definido como variable dicotómica ( $<50 />80$ lpm o 110-130lpm).

- Presión arterial sistólica: variable cuantitativa, medida en $\mathrm{mmHg}$, en el momento del ingreso en planta del paciente. A efectos del cálculo de la escala de PREPARE, la cifra de presión arterial sistólica se ha definido como variable dicotómica $(<110 />130$ mmHg o 110-130 mmHg).

- Cifra de hemoglobina: variable cuantitativa, medida en $\mathrm{g} / \mathrm{dL}, \mathrm{y}$ determinada mediante analítica sanguínea, obteniendo la muestra inmediatamente antes del traslado del paciente a quirófano. A efectos del cálculo de la escala de PREPARE, la cifra de hemoglobina se ha definido como variable dicotómica $(<11,5 />17$ g/dL o 11,5-17 g/dL).

- Grado de riesgo anestésico empleando la escala ASA.: definida como variable dicotómica (grados I y II frente a los grados $\geq$ III). 
- Puntuación de la escala PREPARE (136): definida como variable cuantitativa y determinada según la asignación de puntos especificada en la tabla 12. A efectos del cálculo de la escala, la puntuación también se ha definido como variable categórica En base a la puntuación obtenida por cada individuo, se le asigna a una determinada categoría de riesgo: bajo (de 0 a 5 puntos), medio (de 6 a 9 puntos) y alto (10 o más puntos). [Figura 10].

En segundo lugar, se seleccionaron los individuos de la muestra sometidos a la técnica de DPC, constituyendo el llamado SDPC. En este subgrupo, se volvieron a registrar las variables demográficas y aquellas que forman parte de la escala PREPARE (136) ya mencionadas previamente. Además, se incluyeron las siguientes variables relacionadas con la segunda escala que se ha analizado en este trabajo.

- Variables incluidas en la escala Wiltberger (137):

- IMC: registrada como variable cuantitativa, determinada por la fórmula de Quetelet en $\mathrm{Kg} / \mathrm{m}^{2}$, y en el momento del ingreso hospitalario del paciente, la tarde previa a la intervención quirúrgica. A efectos del cálculo de la escala, el IMC se ha definido como variable categórica tal y como se indica: $<25 \mathrm{Kg} / \mathrm{m}^{2}, \geq 25-30 \mathrm{Kg} / \mathrm{m}^{2},>30 \mathrm{Kg} / \mathrm{m}^{2}$.

- Grado de riesgo anestésico empleando la escala ASA, definida como variable dicotómica (grados I y II frente a los grados $\geq$ III).

- Patología cardiovascular: variable cualitativa dicotómica (sí/no). Definida como la presencia de hipertensión arterial, arteriopatía periférica, enfermedad coronaria, infarto de miocardio o arritmias., según Wiltberger et al. 
- Patología respiratoria: variable cualitativa dicotómica (sí/no). Definida como la presencia de EPOC, asma, TEP, síndrome de apnea-hipoapnea del sueño.

- Puntuación de la escala de Wiltberger (137): definida como variable cuantitativa y determinada según la asignación de puntos especificada en la tabla 13. A efectos del cálculo de la escala, la puntuación también se ha definido como variable categórica En función de la puntuación obtenida por cada paciente, se le asigna a una determinada categoría de riesgo: bajo ( 0 a 4 puntos), medio (5 a 10 puntos) y alto (11 a 16 puntos) [Figura 11 ].

\subsection{3.- VARIABLES INTRAOPERATORIAS}

- Esplenectomía: variable cualitativa dicotómica (sí/no). Sólo aplicable en PD y DPT

- Reconstrucción: variable cualitativa dicotómica (tipo Child/Y de roux), sólo aplicable en DPC.

- Anastomosis Braun: variable cualitativa dicotómica (sí/no), sólo aplicable en DPC.

- Transfusión de concentrado de hematíes intraoperatoria: variable cualitativa dicotómica (sí/no).

- Tipo de abordaje: variable cualitativa dicotómica (laparoscópico/laparotómico).

- Conversión a laparotomía: variable cualitativa dicotómica (sí/no), sólo aplicable en aquellos cuya variable de tipo de abordaje fue laparoscopia. 
- Drenaje: variable cualitativa dicotómica (sí/no). Se dará el porcentaje de utilización del mismo en toda la SG y en el SDPC como en el resto de variables.

\subsection{4.- VARIABLES POSTOPERATORIAS}

En ambos apartados, (SG y SDPC) se recogieron las complicaciones postoperatorias registradas hasta los 30 DPO y catalogadas según su severidad empleando la clasificación de Dindo-Clavien (114) [tabla 4]. A partir de esta variable se establecerán dos nuevas variables dicotómicas denominadas "complicaciones $\geq$ IIIa" (incluye grados IIIa en adelante de la mencionada clasificación) y "complicaciones $\geq$ IIIb" (incluye grados IIIb en adelante), puesto que son los tipos de complicaciones que predicen las escalas PREPARE y Wiltberger, respectivamente. Esta variable será tenida en cuenta hasta los 30 DPO. Estando ingresado el paciente o haya sido dado de alta y reingrese de nuevo, o incluso preguntándoselo en consulta posteriormente si ha estado ingresado en otro centro.

- Estancia postoperatoria en la Unidad de Reanimación postquirúrgica: variable cuantitativa discreta, definida por DPO que el paciente pernocta en la Unidad de Reanimación postquirúrgica.

- Tolerancia: variable cualitativa dicotómica (sí/no).

- Días hasta tolerancia: variable cuantitativa discreta, definida por el número de DPO que el paciente está en dieta absoluta.

- NPT: variable cualitativa dicotómica (sí/no).

- Días de NPT: variable cuantitativa discreta.

- Complicaciones: variable cualitativa dicotómica (sí/no). 
- FPPO: clasificada como variable cualitativa dicotómica (sí/no). A partir de ella se definirá otra variable cualitativa ordinal clasificada como fístula bioquímica, grado B o grado $\mathrm{C}$, definida según los criterios de ISGPS (115). [Figura 8].

- FPCR: variable cualitativa dicotómica. (FPPO grado B/C).

- RVG: clasificada como variable cualitativa dicotómica (sí/no). A partir de ella se definirá otra variable cualitativa ordinal clasificada como grado A, grado B o grado C, definida según los criterios de ISGPS (121). [Tabla 7]

- HPP: clasificada como variable cualitativa dicotómica (sí/no) y variable cualitativa ordinal clasificada como grado A, grado B o grado C. Definida según los criterios de ISGPS (128). [Tabla 10]

- IHQ: variable cualitativa dicotómica (si/no).

- Reintervención: variable cualitativa dicotómica (sí/no).

- Estancia postoperatoria global: variable cuantitativa discreta, definida por DPO que el paciente pernocta en el hospital desde el día de la intervención quirúrgica hasta el alta.

\section{5.- ANÁLISIS ESTADÍSTICO}

\subsection{1.- RECOGIDA Y MANEJO DE LOS DATOS}

Los datos fueron recogidos e introducidos de forma anónima, encriptados y disociados de la información clínica mediante un código de identificación del paciente, en una base de datos realizada por el programa estadístico SPSS ${ }^{\circ}$ versión 18.0 (IBM 
Corporation ${ }^{\circledR}$, Armonk, New York, Estados Unidos). Se definió un código identificación para cada paciente con su custodia en un archivo protegido mediante contraseña personal. Los datos introducidos en la base de datos fueron anónimos. El fichero fue custodiado en el HURH y se mantuvo hasta la finalización del estudio. Tras la depuración de los datos, estos fueron analizados utilizando el mismo programa estadístico. Dado que la recogida de datos fue prospectiva, las pérdidas se limitaron al 5\%, de modo que la pérdida de valores superior a esta cifra en una determinada variable fue considerada como un posible sesgo del estudio e informada en el análisis de resultados.

\subsection{2.- ESTADÍSTICA DESCRIPTIVA}

La normalidad de las variables cuantitativas se estableció con la prueba de Kolmogorov-Smirnov. Las variables cuyas distribuciones no se alejaron de la normalidad fueron descritas como media \pm desviación estándar y las que presentaron distribuciones alejadas de la normalidad como mediana y rango intercuartílico (RIQ). Por su parte, las variables cualitativas y cuantitativas discretas fueron descritas mediante las frecuencias absolutas y relativas (porcentajes) de sus categorías. Finalmente, se utilizaron histogramas de frecuencia para representar las variables cuantitativas y gráficos de barras o de sectores para las cualitativas.

\subsection{3.- ESTADÍSTICA INFERENCIAL}

Para estudiar la asociación entre variables cualitativas se utilizaron las pruebas de Chi-cuadrado, aplicando la Chi-cuadrado de Pearson para las tablas 2 por 2, la razón de verosimilitudes para más de 2 categorías, o el test exacto de Fisher para una frecuencia 
esperada inferior a 5 en más del $20 \%$ de las casillas. Se presentó, igualmente, el dato de riesgo relativo $(\mathrm{RR})$ entre grupos.

Para estudiar las diferencias entre medias independientes, para 2 grupos, se utilizó la prueba de la T de Student o la U de Mann-Whitney, dependiendo de las condiciones de aplicación (alejamiento o no de la distribución normal).

También se reflejó respectivamente en ambos apartados (SG y SDPC), la evaluación de la fiabilidad de estas dos escalas predictoras de complicaciones. Para ello se aplicaron los siguientes test estadísticos:

- Curva Receiver Operating Characteristic (ROC), indicando el área bajo la curva (AUC, por sus siglas en inglés: Area Under Curve) obtenida, como método de discriminación.

- Test de "bondad de ajuste" de Hosmer-Lemeshow, aportando la proporción de pacientes correctamente asignados y el nivel de significación estadística de la prueba, como método de calibración.

Se realizó un análisis bivariante entre las categorías de riesgo de la escala PREPARE en la SG y la escala PREPARE y escala Wiltberger en el SDPC y las variables resultado del estudio, utilizando el test estadístico de la razón de verosimilitud.

Los resultados se expresaron con sus correspondientes intervalos de confianza al 95\% (IC 95\%) cuando procediese, y el nivel de significación estadística, para todas las pruebas citadas, se considerará con un valor de $\mathrm{p} \leq 0,05$. 


\section{6.- ÉTICA Y ASPECTOS LEGALES}

Este estudio se ajusta a los requisitos de Declaración de Helsinki de 1964 (última enmienda en octubre de 2013 durante la 64ª asamblea de la Asociación Médica Mundial). No se ha recibido ninguna beca ni financiación de otro tipo para la realización del mismo. No existe ningún otro tipo de conflicto de intereses. El estudio ha sido aprobado por el Comité de ética asistencial del HURH.

\section{7.- BÚSQUEDA BIBLIOGRÁFICA}

La búsqueda bibliográfica se ha realizado en la base de datos PubMed/MEDLINE, cuyos datos han sido desarrollados y se mantienen actualizados por la National Library of Medicine de los Estado Unidos de América.

La búsqueda inicial se planteó en base a el pilar fundamental del trabajo, que son los trabajos originales que describen las escalas de PREPARE (136) y Wiltberger (137).

Las búsquedas asociadas se plantearon en base a términos libres y la definitiva mediante términos MeSH (Medical Subject Headings), utilizando los operadores boléanos, los límites de tiempo y el filtro de idioma para confeccionar la estrategia más adecuada. Los términos MeSH empleados fueron: pancreatic surgery, postoperative complications, morbidity, pancreatic fistula, gastric delayed emptying, hemorrhage postpancreatectomy. Los idiomas seleccionados fueron inglés y español. 


\section{RESULTADOS}


Francisco Javier Tejero Pintor 


\section{RESULTADOS}

En el periodo de estudio (1 de enero de 2015 hasta el 31 de mayo de 2019), tras aplicar los criterios de inclusión y exclusión descritos previamente, se han realizado 97 resecciones pancreáticas mayores, todas ellas incluidas para el análisis de la SG.

\section{1.- SERIE GLOBAL}

\subsection{1.- ANÁLISIS DESCRIPTIVO}

\subsubsection{1.- VARIABLES DEMOGRÁFICAS}

En la SG se obtuvo un tamaño muestral de 97 enfermos en el periodo de tiempo indicado. La variable "sexo" siguió la siguiente distribución: 49 (50,5\%) hombres y 48 (49,5\%) mujeres [Gráfico 1]. La variable “edad” presentó una proporción normal, con una media de $61 \pm 11$ años (rango de 29 a 79 años) [Gráfico 2]. 
Gráfico 1: gráfico de sectores que muestra distribución por sexo en la SG.

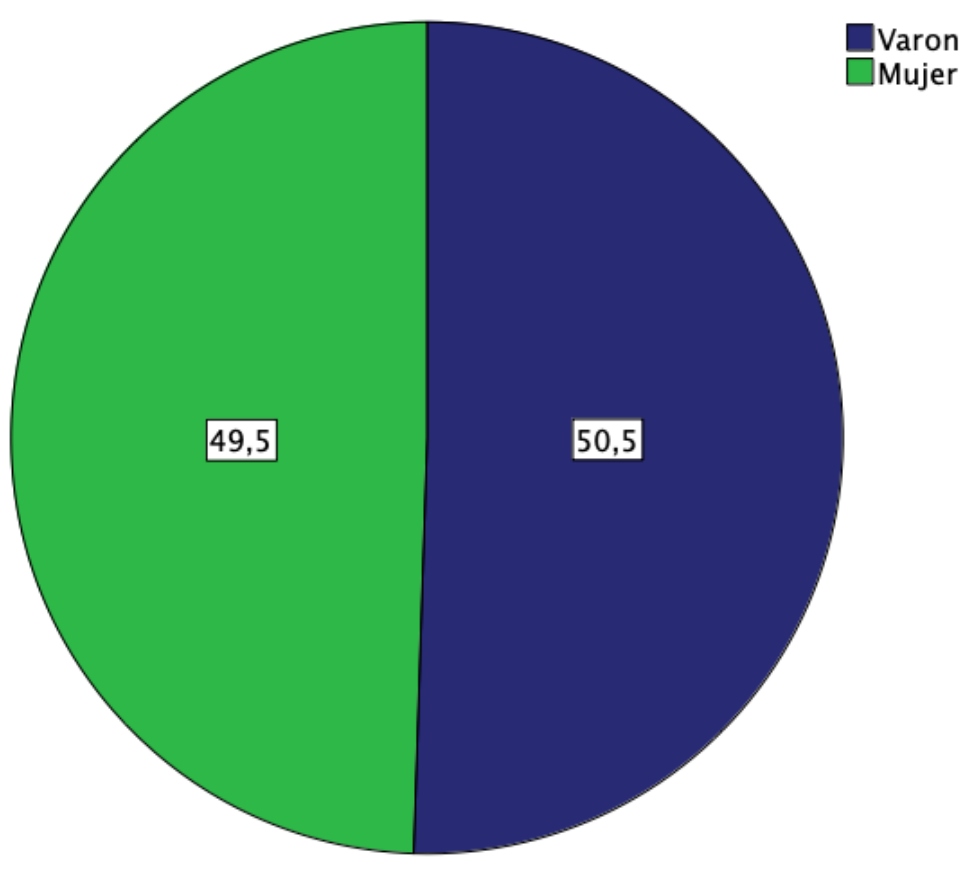

Gráfico 2: histograma que muestra la distribución por edad en la SG.

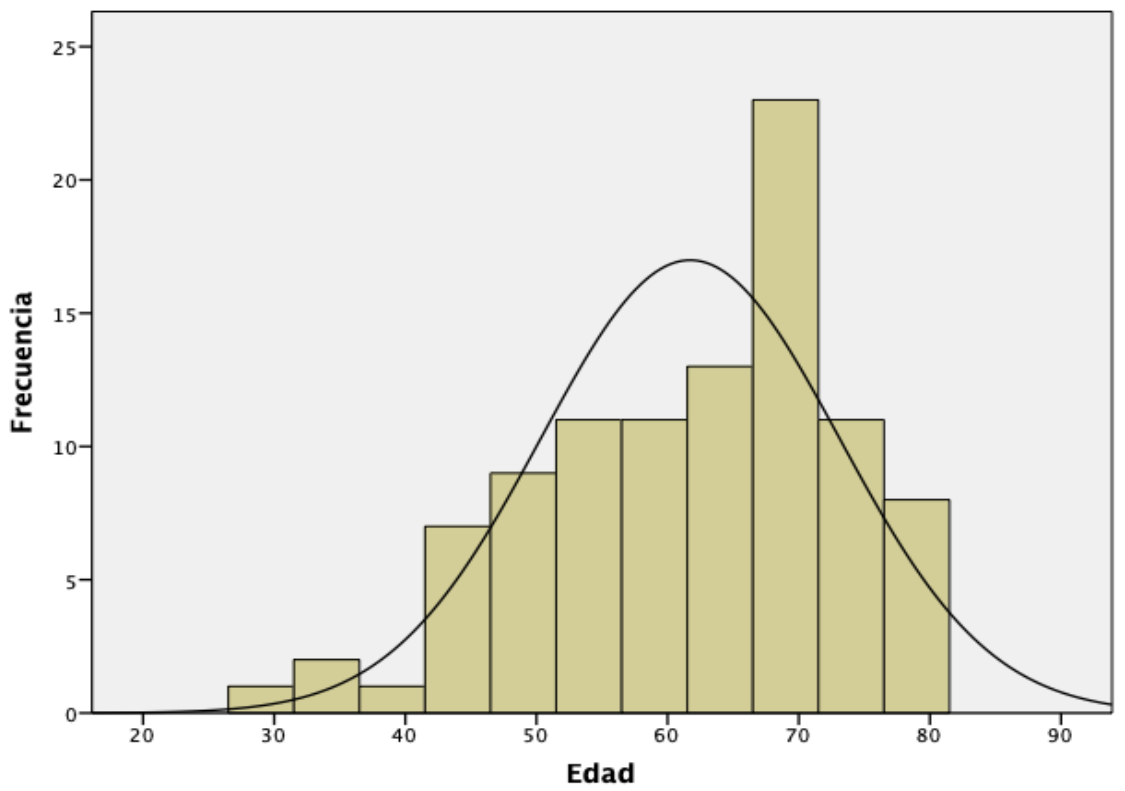




\subsubsection{2.- VARIABLES PREOPERATORIAS}

Los antecedentes médicos personales siguieron la siguiente distribución: el 23,7\% de los pacientes consumían alcohol, 24,7\% tienen tabaquismo, 30,9\% dislipemia, 22,7\% diabetes, 36,1\% hipertensión arterial [Gráfico 3].

Gráfico 3: Diagrama de barras que muestra la distribución porcentual de consumo de alcohol, tabaquismo, dislipemia, diabetes e hipertensión arterial

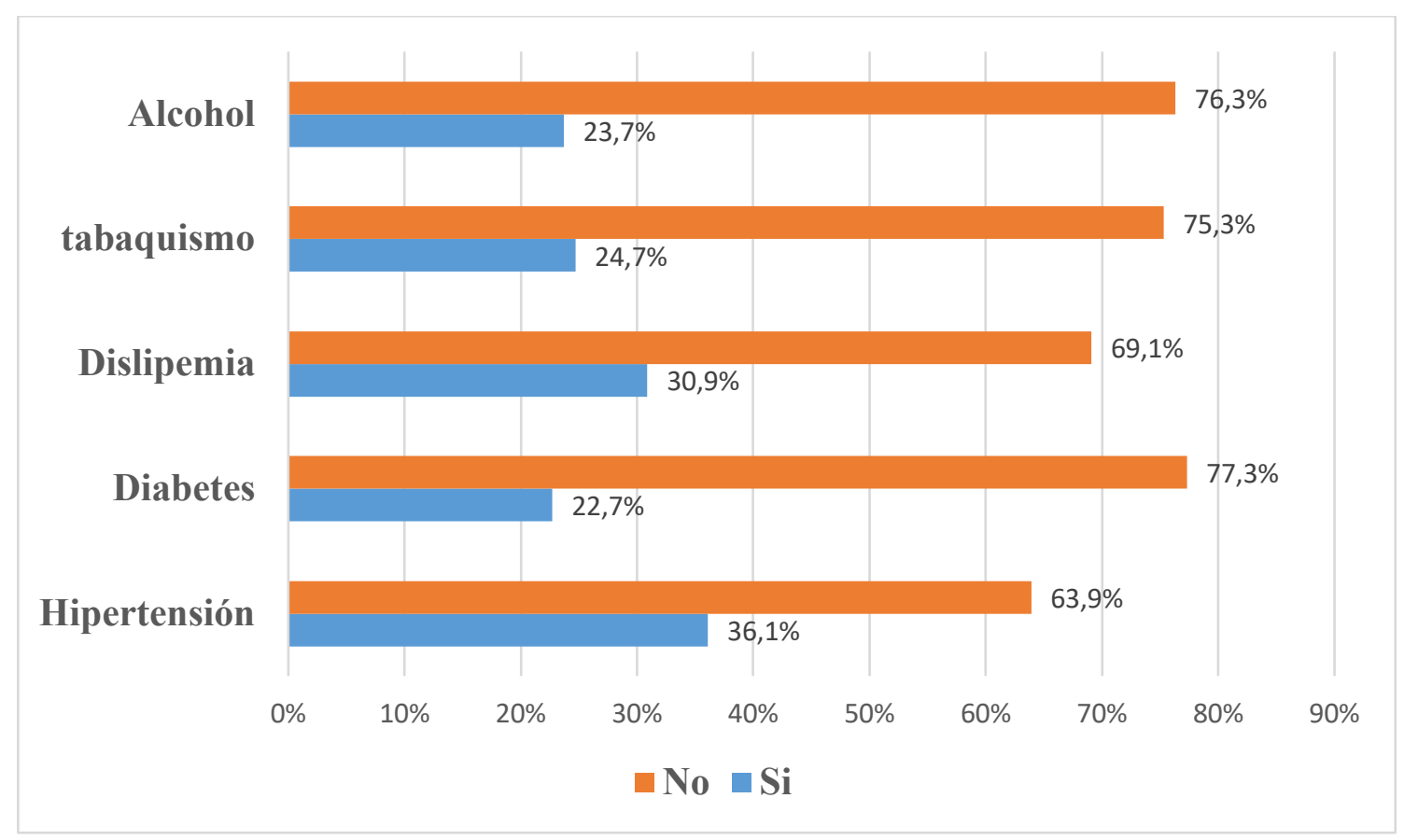

En el 63,9\% de los pacientes se colocó prótesis biliar preoperatoria. Analizando únicamente a los pacientes que fueron sometidos a DPC y DPT esta cifra asciende al $78,5 \%$. 
En el análisis descriptivo de las variables clínicas y analíticas incluidas en la escala PREPARE, se obtuvieron los siguientes resultados:

- Cifra media de albúmina: siguió una distribución normal con una media de 3,7 $\pm 0,4 \mathrm{~g} / \mathrm{dL}$ (rango de 2,6 a 5,2 g/dL).

- Tipo de procedimiento: en ningún paciente de los 97 (0\%) se realizó cirugía pancreática de urgencia.

- Técnica quirúrgica: en 74 pacientes $(76,3 \%)$ se practicó una DPC y en los 23 restantes $(23,7 \%)$, una PD o una DPT (18,5\% y 5,2\%, respectivamente).

- Patología de origen pancreático: 64 pacientes $(66 \%)$ presentaban patología puramente pancreática, frente a los 33 restantes (34\%) que era patología de otro origen.

- Cifra media de frecuencia cardiaca: siguió una distribución normal con una media de 77,1 $\pm 12,7 \mathrm{lpm}$ (rango de 44 a $108 \mathrm{lpm}$ ).

- Cifra media de presión arterial sistólica: siguió una distribución normal con una media de 128,4 $\pm 17,1 \mathrm{mmHg}$ (rango de 98 a $186 \mathrm{mmHg}$ ).

- Cifra media de hemoglobina: siguió una distribución normal con una media de $13,2 \pm 1,5 \mathrm{~g} / \mathrm{dL}$ (rango de 9,1 a 17,2 g/dL).

- Grado de riesgo anestésico ASA: 65 pacientes (67\%) fueron grados I y II (8,2\% y 58,8\%, respectivamente), y los 32 restantes fueron ASA-III (33\%). No se identificó ningún caso de ASA-IV.

La distribución de la asignación de puntos, siguiendo los criterios establecidos por la escala PREPARE (136) en cada una de las 8 variables, se muestra en la tabla 15. Asimismo, la puntuación en dicha escala para la SG siguió una distribución no normal y 
se obtuvo una mediana de 5 puntos y un RIQ de 4-7 puntos. Por ultimo, la proporción según las 3 categorías de riesgo de la escala PREPARE fue la siguiente [Gráfico 4]:

- Riesgo bajo (0-5 puntos): 55 pacientes $(56,7 \%)$.

- Riesgo medio (6-9 puntos): 29 pacientes (29,9\%).

- Riesgo alto (10-17 puntos): 13 pacientes $(13,4 \%)$.

Tabla 15. Distribución de cada variable incluida en la escala PREPARE en la SG de pacientes, notificando la proporción de casos en que suma puntos.

\begin{tabular}{|c|c|c|c|}
\hline VARIABLE & DATO & PUNTUACIÓN & $\begin{array}{l}\text { PROPORCIÓN } \\
\text { DE PACIENTES }\end{array}$ \\
\hline \multirow{2}{*}{$\begin{array}{l}\text { Albúmina } \\
\text { plasmática }\end{array}$} & $\geq 3,5 \mathrm{~g} / \mathrm{dL}$ & & \\
\hline & $<3,5 \mathrm{~g} / \mathrm{dL}$ & 5 puntos & $\begin{array}{c}24 \text { pacientes } \\
(24,7 \%)\end{array}$ \\
\hline \multirow{2}{*}{$\begin{array}{c}\text { Cirugía } \\
\text { programada }\end{array}$} & Sí & & \\
\hline & No (urgente) & 4 puntos & $\begin{array}{c}\text { Ningún paciente } \\
(0 \%)\end{array}$ \\
\hline \multirow{2}{*}{$\begin{array}{c}\text { Técnica } \\
\text { quirúrgica }\end{array}$} & Otras técnicas & & \\
\hline & $\begin{array}{l}\text { DPC o resección } \\
\text { de cabeza de } \\
\text { páncreas con } \\
\text { preservación del } \\
\text { duodeno }\end{array}$ & 2 puntos & $\begin{array}{c}74 \text { pacientes } \\
(76,3 \%)\end{array}$ \\
\hline \multirow{2}{*}{$\begin{array}{c}\text { Patología de } \\
\text { origen } \\
\text { pancreático }\end{array}$} & Sí & & \\
\hline & No & 2 puntos & $\begin{array}{c}33 \text { pacientes } \\
(34 \%)\end{array}$ \\
\hline \multirow{2}{*}{$\begin{array}{c}\text { Frecuencia } \\
\text { cardíaca }\end{array}$} & 50-80 lpm & & \\
\hline & $<50$ o $>80 \mathrm{lpm}$ & 2 puntos & $\begin{array}{c}41 \text { pacientes } \\
(42,3 \%)\end{array}$ \\
\hline \multirow{2}{*}{$\begin{array}{c}\text { Presión } \\
\text { arterial } \\
\text { sistólica } \\
\end{array}$} & $110-130 \mathrm{mmHg}$ & & \\
\hline & $\begin{array}{c}<110 \mathrm{o}>130 \\
\mathrm{mmHg}\end{array}$ & 2 puntos & $\begin{array}{c}46 \text { pacientes } \\
(47,4 \%)\end{array}$ \\
\hline \multirow{2}{*}{$\begin{array}{l}\text { Hemoglobina } \\
\text { en sangre }\end{array}$} & $11,5-17 \mathrm{~g} / \mathrm{dL}$ & & \\
\hline & $<11,5$ o $>17 \mathrm{~g} / \mathrm{dL}$ & 1 punto & $\begin{array}{c}13 \text { pacientes } \\
(13,4 \%)\end{array}$ \\
\hline \multirow{2}{*}{$\begin{array}{c}\text { Riesgo } \\
\text { anestésico } \\
\text { (escala ASA) }\end{array}$} & $\leq \mathrm{II}$ & & \\
\hline & $\geq \mathrm{III}$ & 1 punto & $\begin{array}{l}32 \text { pacientes } \\
(33 \%)\end{array}$ \\
\hline
\end{tabular}


Gráfico 4. Gráfico de sectores que muestra la distribución de pacientes de la muestra en el apartado SG según las categorías de riesgo de la escala PREPARE (bajo, medio y alto).

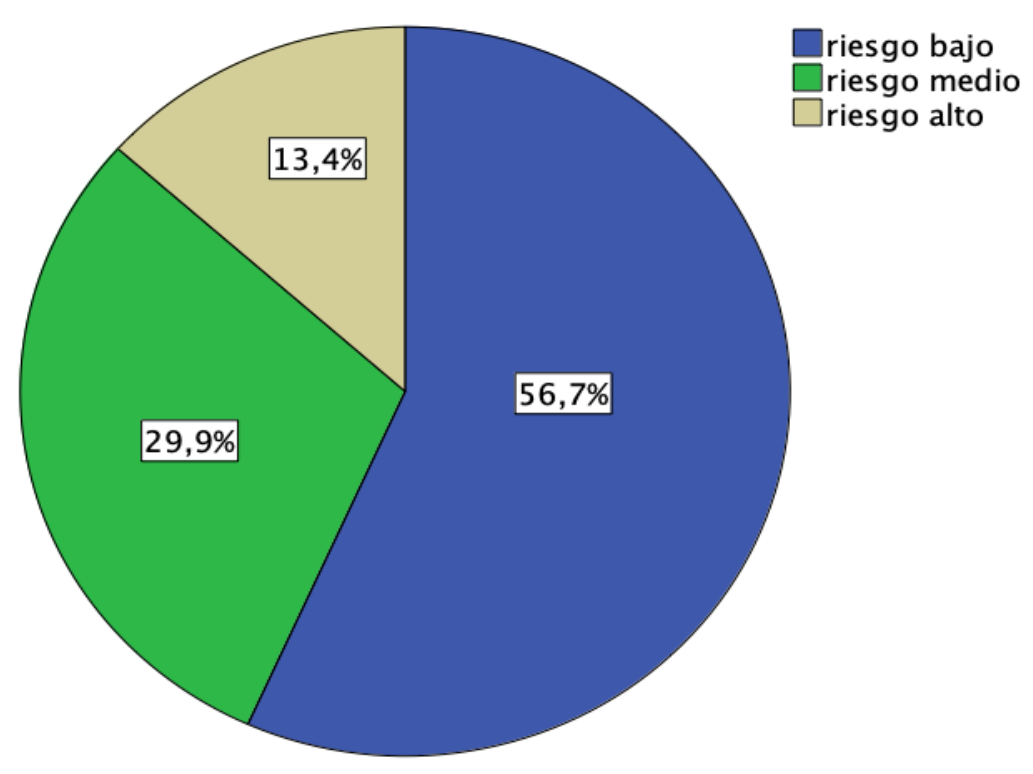

\subsubsection{3.- VARIABLES INTRAOPERATORIAS}

La distribución del tipo de resecciones pancreáticas realizadas durante el mencionado periodo de recogida se muestra en la figura 12. Las variables intraoperatorias de esta SG siguieron la siguiente distribución: el 20,6\% de los pacientes precisaron esplenectomía (todas ellas fueron en pacientes a los que se les había realizado una PD o DPT). Seleccionando únicamente estos dos tipos de resecciones, en el $87 \%$ de ellas se realizó esplenectomía asociada. El 8,2\% necesitaron trasfusión de concentrado de hematíes. El 5,2\% de los pacientes fueron intervenidos con abordaje laparoscópico a todos ellos se les realizó PD. En el 20\% de estos últimos fue necesaria la conversión a cirugía abierta. En el 84,5\% de los pacientes se dejó drenaje intraabdominal. Excluyendo a los 5 pacientes a los que se realizó DPT, en los que no se dejó drenaje intraabdominal, el porcentaje de enfermos a los que se les dejó drenaje intraabdominal asciende al 89,1\%. 
Figura 12: Resecciones pancreáticas y gestos quirúrgicos intraoperatorios asociados.

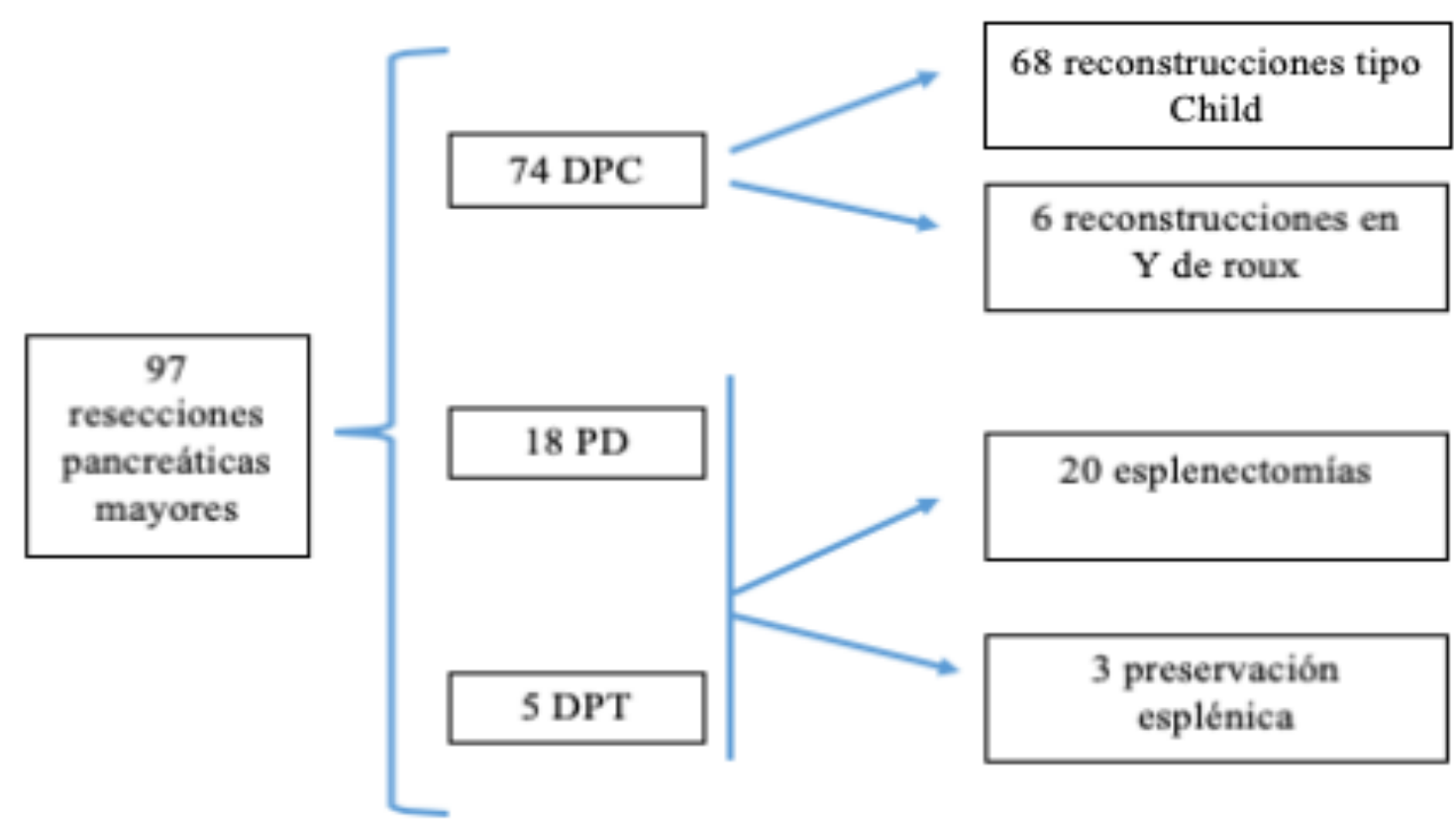

\subsubsection{4.- VARIABLES POSTOPERATORIAS}

Todos los enfermos acudieron por protocolo clínico a la unidad de reanimación postquirúrgica después de la resección pancreática. La estancia postoperatoria en dicha unidad siguió una distribución no normal y tuvo una mediana de 3 días y un RIQ de 2-3 días.

Todos los pacientes menos uno, consiguieron tolerancia oral durante el postoperatorio, obteniendo una distribución no normal con una mediana de 4 días y un RIQ de 3-7 días para la variable de días hasta tolerancia. En cuanto al uso de NPT, en el $85,5 \%$ de los pacientes se instauró después de la cirugía. La mediana de días de administración de NPT en estos pacientes fue de 6 con un RIQ de 5-10 días. 
El 75,3\% de los pacientes presentó algún tipo de complicación en el postoperatorio. La distribución de estas complicaciones según el grado de gravedad en base a clasificación de Dindo et al. (114) se muestra en el gráfico 5. A su vez el 26,8\% de los enfermos tuvieron complicaciones graves (Clavien $\geq$ IIIa) aplicando la definición empleada en la escala PREPARE desarrollada por Uzunoglu et al. (136).

Gráfico 5. Gráfico de barras que muestra la distribución de complicación según los grados de gravedad de Dindo et al. (114).

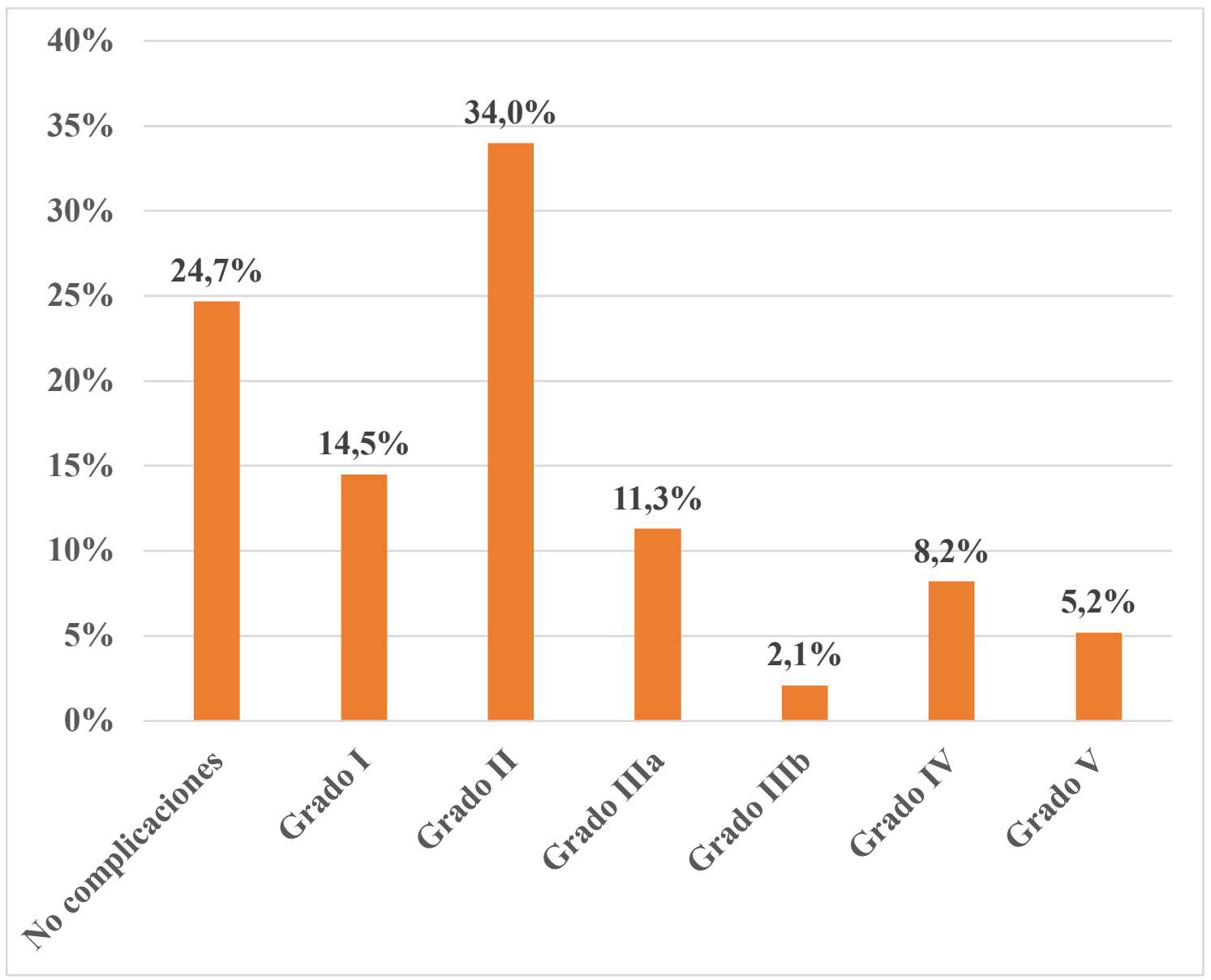


Las complicaciones específicas que se obtuvieron tras pancreatectomía fueron las siguientes:

- El 28,2\% de los enfermos (excluidos los 5 enfermos a los que se realizó DPT), desarrollaron FPPO. El 10,8\% tuvieron FPCR. El gráfico 6 muestra la distribución según los grados de gravedad.

- El 26,8\% de los pacientes desarrollaron RVG, la distribución según los grados de gravedad puede observarse en el gráfico 7.

- El 18,6\% de los pacientes desarrollaron HPP, la distribución según los grados de gravedad puede observarse en el gráfico 8.

Gráfico 6. Gráfico de barras que muestra la distribución según los grados de gravedad de FPPO (115).

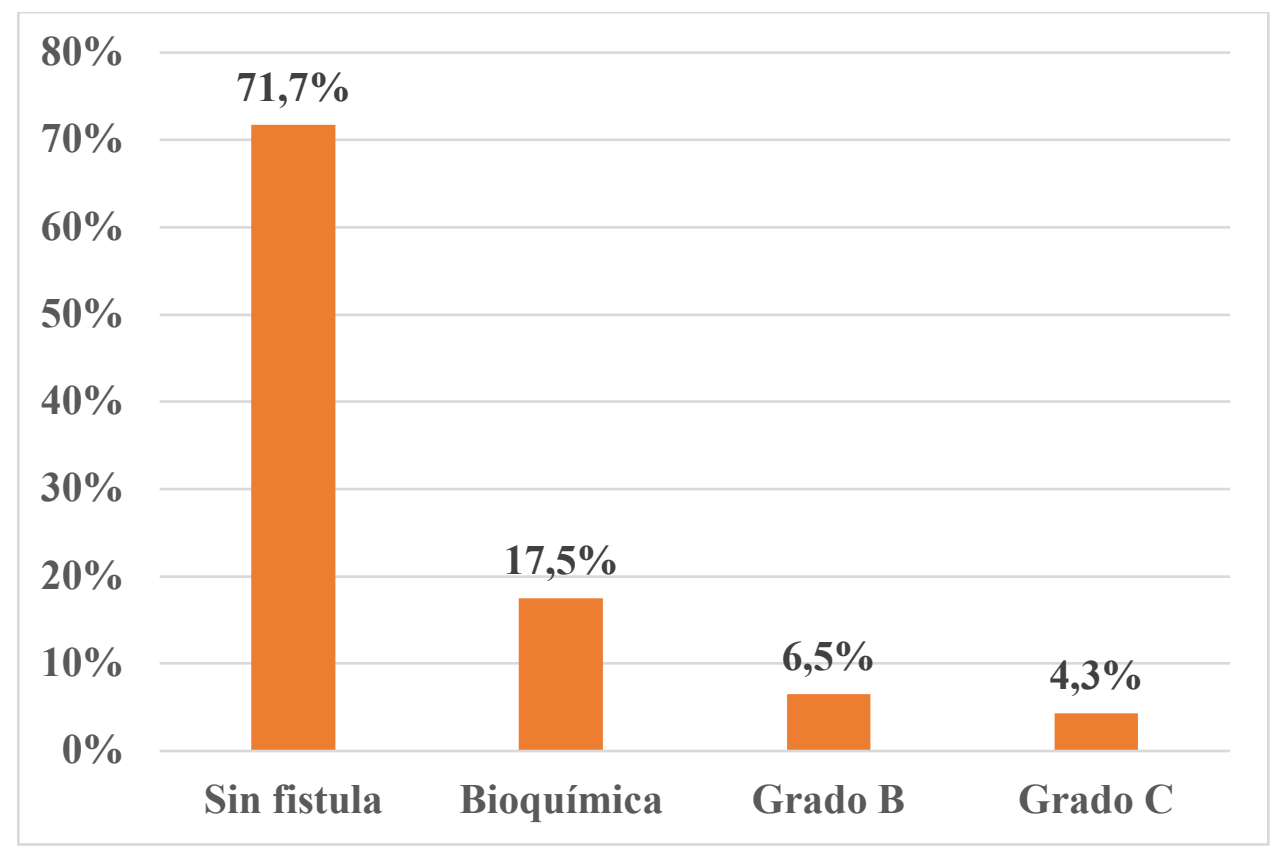


Gráfico 7. Gráfico de barras que muestra la distribución según los grados de gravedad de RVG (121).

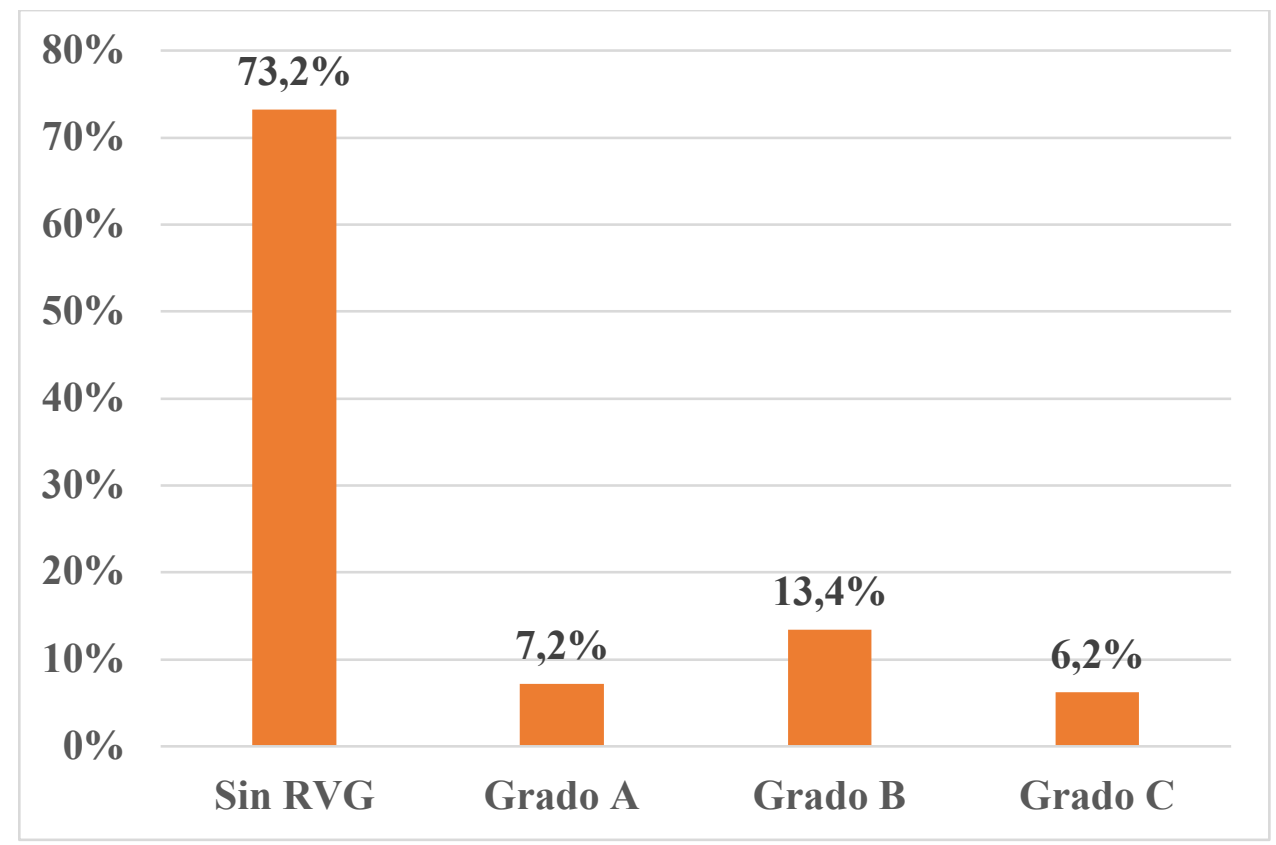

Gráfico 8. Gráfico de barras que muestra la distribución según los grados de gravedad de HPP (128).

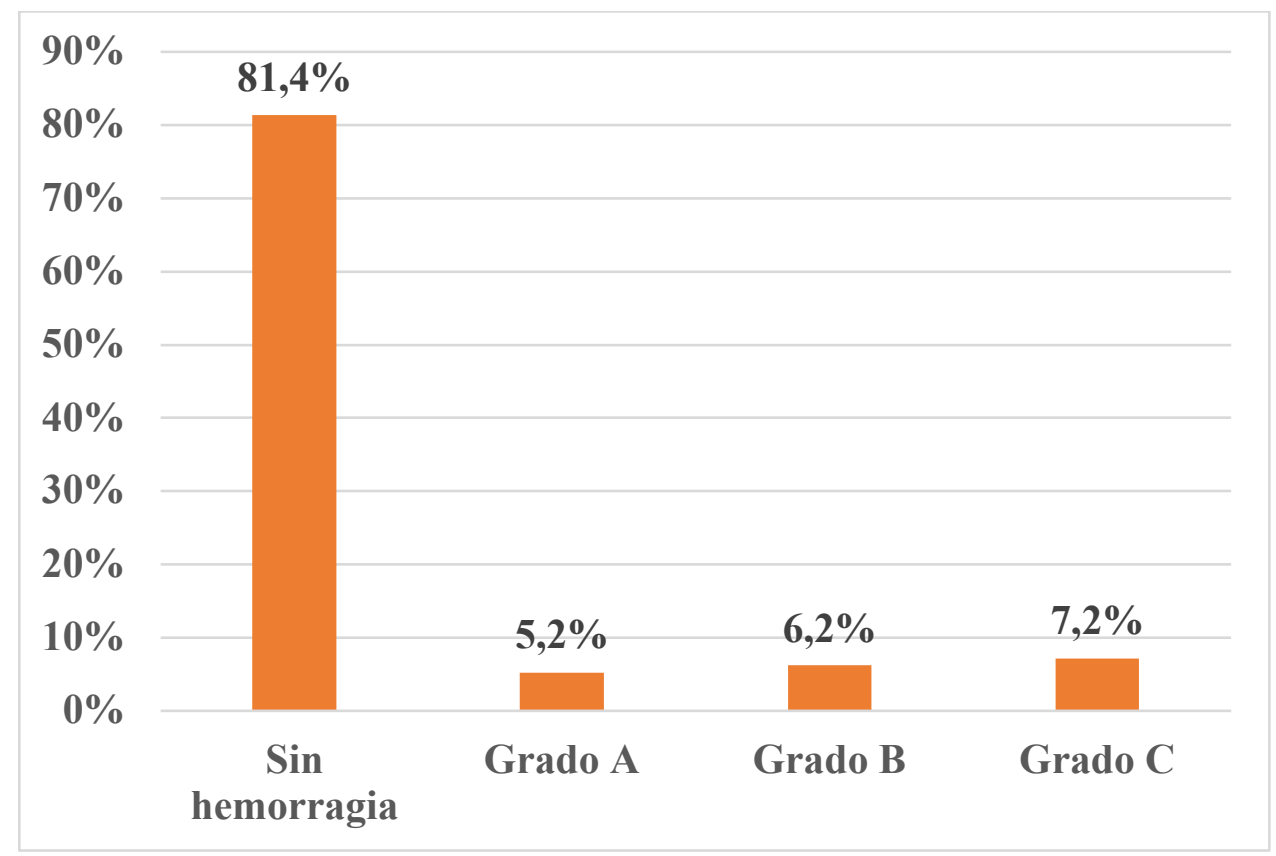


La tasa de IHQ fue $17,5 \%$. En el $8,2 \%$ de los pacientes de la SG fue necesaria la reintervención quirúrgica, siendo, la causa de dicha reintervención la que se muestra en el gráfico 9.

La estancia postoperatoria global, siguió una distribución no normal con una mediana de 11 días y un RIQ de 8-14 días.

Gráfico 9. Gráfico de sectores que muestra la causa de reintervención quirúrgica en el $8,2 \%$ de los pacientes en que fue necesaria.

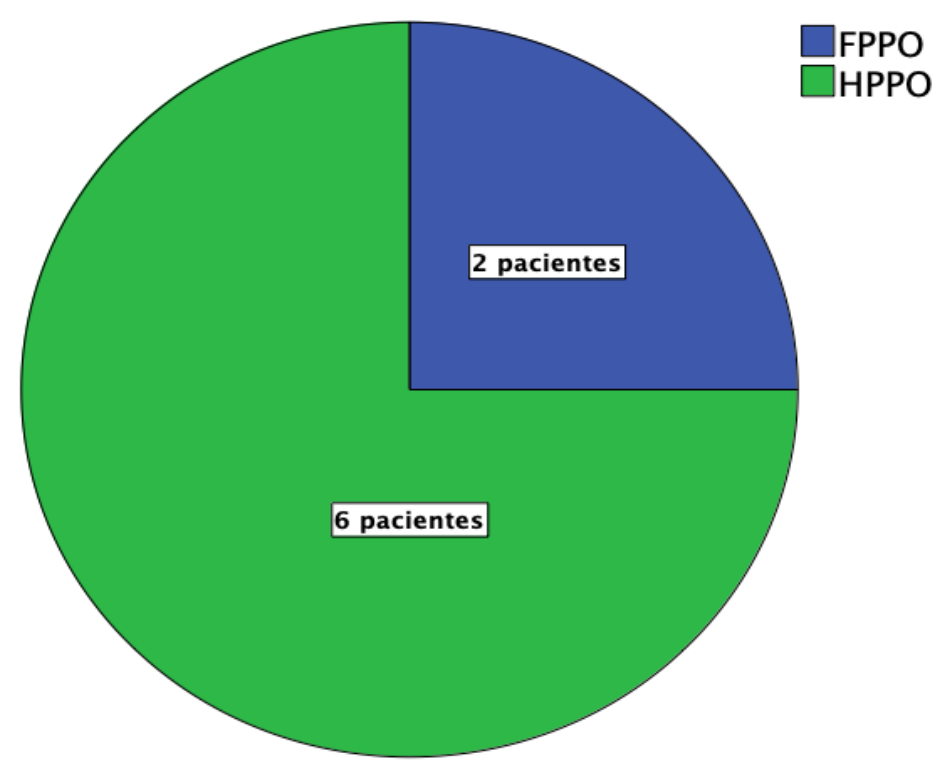

\subsection{2.- ANÁLISIS INFERENCIAL}

Tras establecer la proporción de individuos con complicaciones graves (Clavien $\geq \mathrm{IIIa}$ ) en esta SG en cada una de las categorías de riesgo de la escala PREPARE, se obtiene la siguiente distribución que mostró diferencias estadísticamente significativas $(\mathrm{p}=0,002)$. [gráfico 10] 
- Riesgo bajo: 10 pacientes con complicaciones graves sobre 55 (18,2\%).

- Riesgo medio: 7 pacientes con complicaciones graves sobre $29(24,1 \%)$.

- Riesgo alto: 9 pacientes con complicaciones graves sobre $13(69,2 \%)$.

Gráfico 10: Gráfico de barras que muestra la proporción de pacientes con complicaciones graves (Clavien $\geq$ IIIa) en cada una de las categorías de la escala PREPARE en el análisis de la SG.

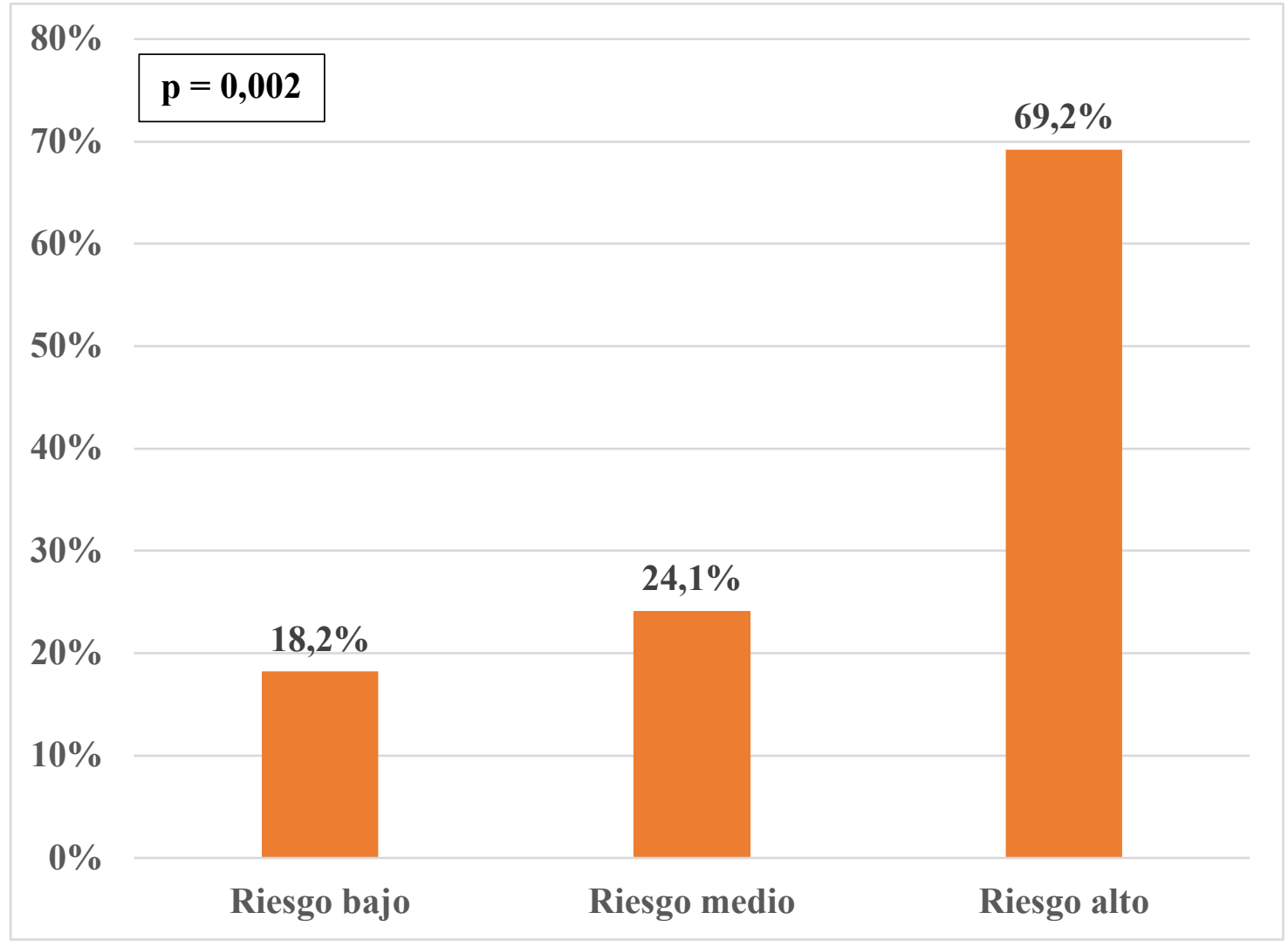

Para comprobar estadísticamente la fiabilidad de este índice, se notificó un $\mathrm{AUC}=0,671(\mathrm{IC} 95 \%=0,539-0,802, \mathrm{p}=0,01)$ en la curva $R O C$ [Gráfico 11]. A la luz de los datos anteriormente expuestos, en el análisis de la SG, los pacientes catalogados como de alto riesgo según la escala PREPARE presentan casi 3 veces más probabilidad 
de sufrir morbilidad grave respecto a los pacientes de la categoría de bajo riesgo $(\mathrm{RR}=$ 2,75, IC $95 \%=1,45-5,21)$. Tras aplicar el test de Hosmer-Lemeshow, se obtuvo un porcentaje pronosticado correcto del 78,4\% y una $\mathrm{p}=0,147$

Gráfico 11.: Curva $R O C$ para evaluar la fiabilidad (en términos de discriminación) de la escala PREPARE en la SG.

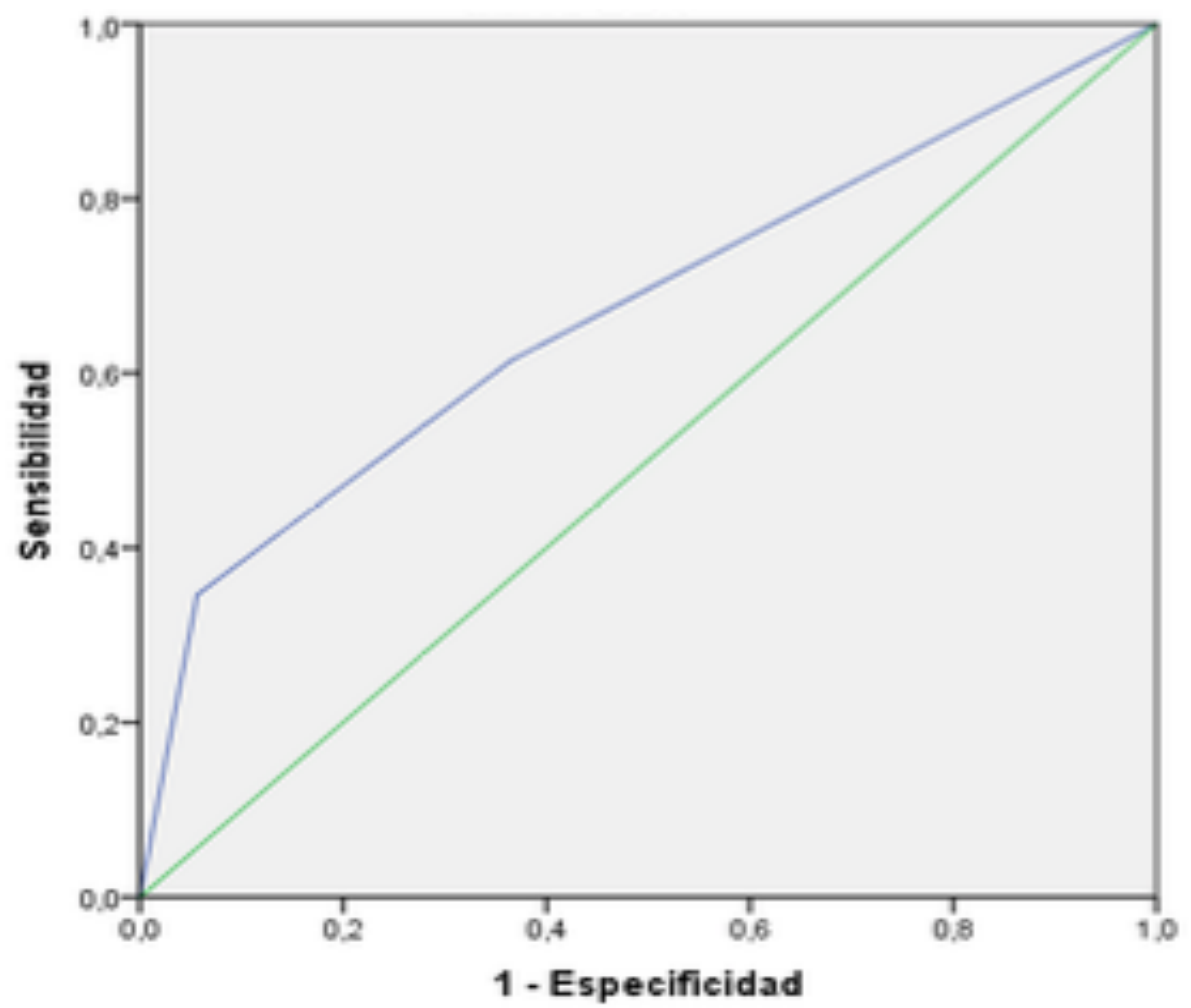

A continuación, en la tabla 16 puede observarse el análisis bivariante entre complicaciones globales, reintervención, FPPO (Excluyendo las DPT), RVG, HPP e IHQ y los grados de riesgo de la escala PREPARE. 


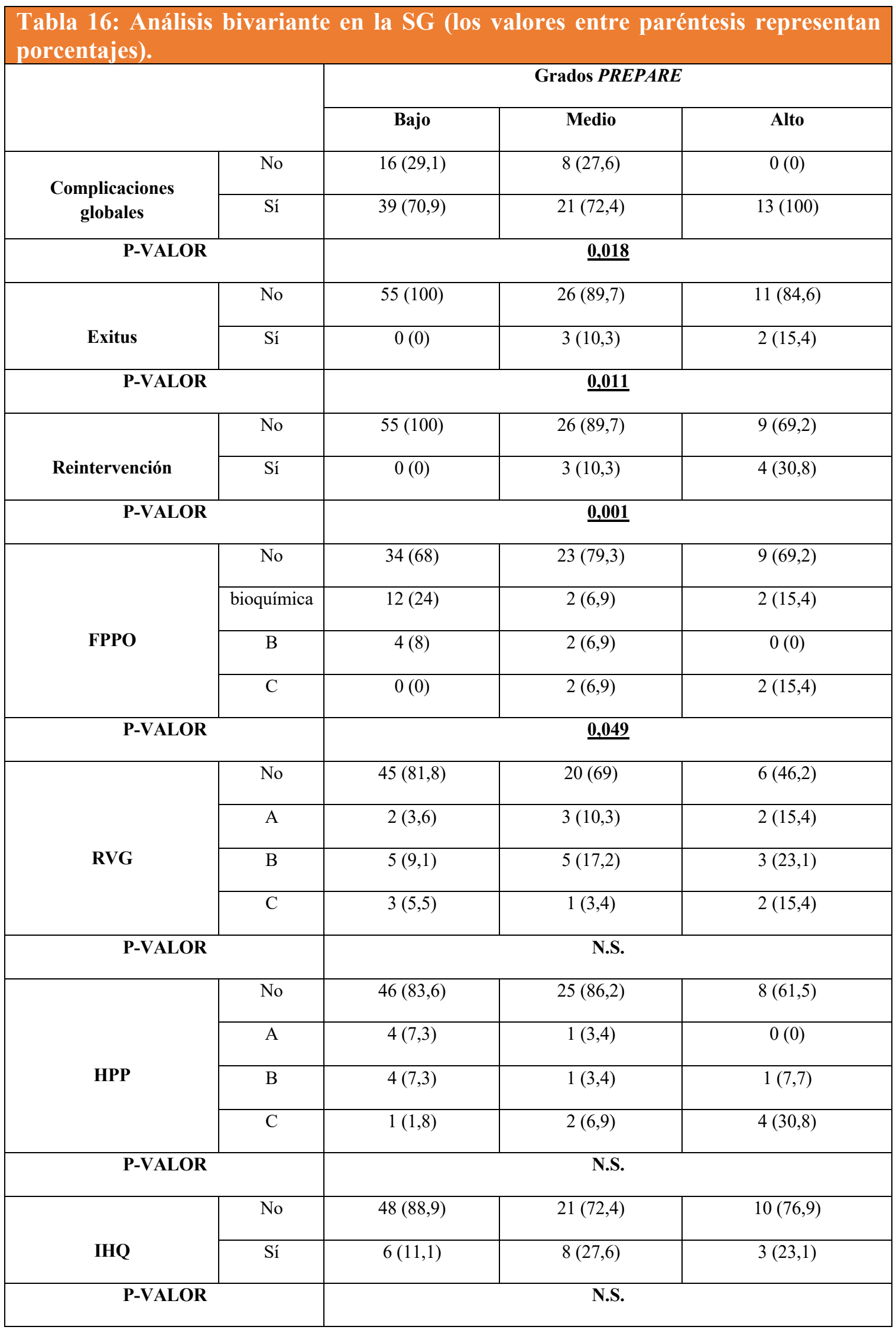




\section{2.- SUBGRUPO DUODENOPANCREATECTOMÍA CEFÁLICA}

Para el análisis del subgrupo DPC, se han seleccionado exclusivamente las 74 DPC de la SG.

\subsection{1.- ANÁLISIS DESCRIPTIVO}

\subsubsection{1.- VARIABLES DEMOGRÁFICAS}

La variable "sexo" siguió la siguiente proporción: $42(56,8 \%)$ hombres y 32 (43,2\%) mujeres [Gráfico 12]. La variable “edad” presentó una distribución normal, con una media de $63 \pm 11$ años (rango de 29 a 79 años) [Gráfico 13].

Gráfico 12: gráfico de sectores que muestra distribución por sexo en la SDPC.

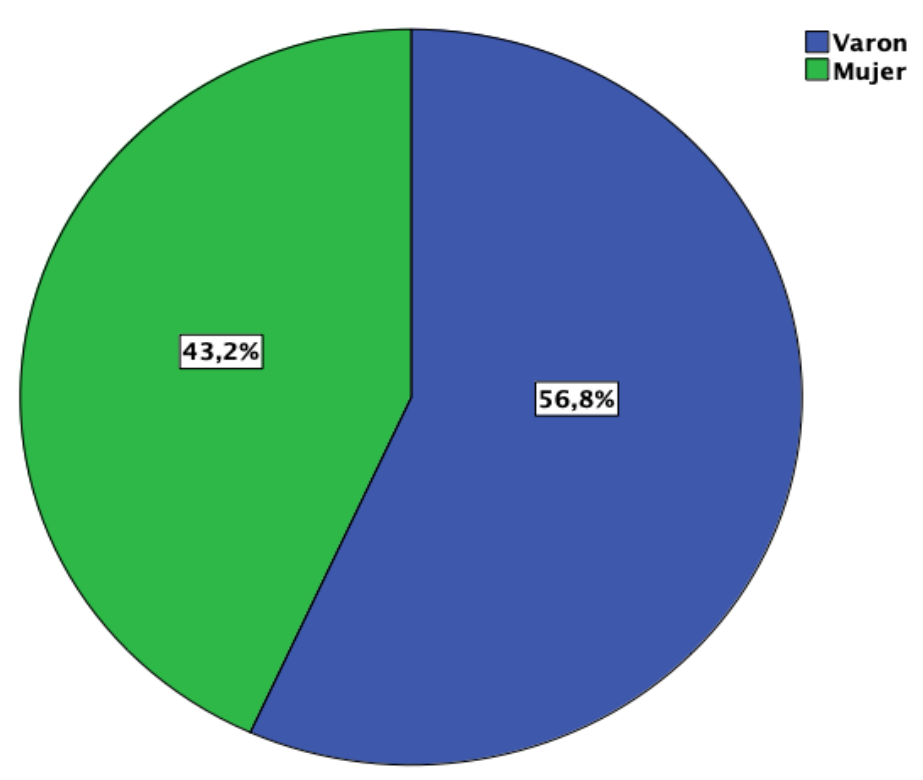


Gráfico 13: histograma que muestra la distribución por edad en el SDPC.

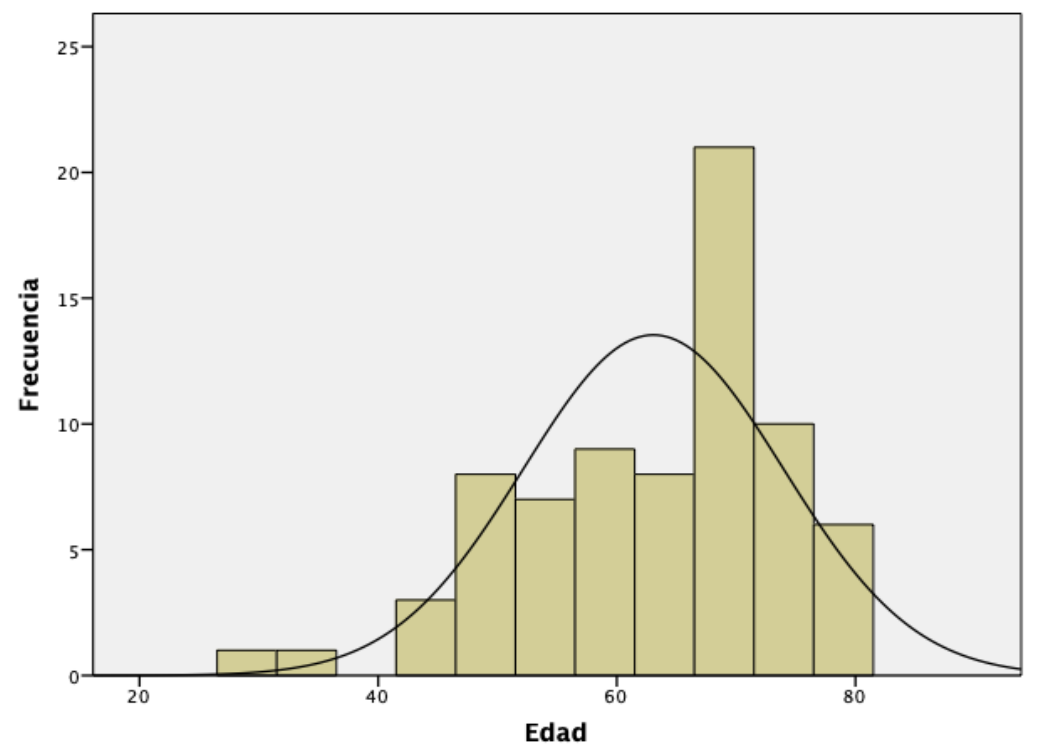

\subsubsection{2.- VARIABLES PREOPERATORIAS}

El 81,1\% de los pacientes se colocó prótesis biliar preoperatoria.

En el análisis descriptivo de las variables clínicas y analíticas incluidas en la escala PREPARE, se obtuvieron los siguientes resultados:

- Cifra media de albúmina: siguió una distribución normal con una media de 3,6 $\pm 0,41 \mathrm{~g} / \mathrm{dL}$ (rango de 2,6 a $4,6 \mathrm{~g} / \mathrm{dL}$ )

- Tipo de procedimiento: en ningún paciente de los 74 (0\%) se realizó cirugía pancreática de urgencia.

- Técnica quirúrgica: en este análisis, del SDPC, todos los pacientes fueron sometidos a dicho procedimiento (100\%). 
- Patología de origen pancreático: 43 pacientes $(58,1 \%)$ presentaban patología puramente pancreática, frente a los 31 restantes $(41,9 \%)$ que era patología de otro origen.

- Cifra media de frecuencia cardiaca: siguió una distribución normal con una media de $75 \pm 11,2$ lpm (rango de 44 a 98 lpm).

- Cifra media de presión arterial sistólica: siguió una distribución normal con una media de $128 \pm 16 \mathrm{mmHg}$ (rango de 98 a $186 \mathrm{mmHg}$ ).

- Cifra media de hemoglobina: siguió una distribución normal con una media de $13 \pm 1,5 \mathrm{~g} / \mathrm{dL}$ (rango de 9,1 a 17,2 g/dL).

- Grado de riesgo anestésico ASA: 46 pacientes (62,2\%) fueron grados I y II (6,8\% y 55,4\%, respectivamente), y los 28 restantes fueron ASA-III (37,8\%, no se identificó ningún caso de ASA-IV).

La asignación de puntos, siguiendo los criterios establecidos por la escala PREPARE (136) en cada una de las 8 variables, se muestra en la tabla 17. Asimismo, la puntuación para el SDPC siguió una distribución normal y se obtuvo una media de 6,5 \pm 2,9 puntos (rango de 2 a 15 puntos). Por ultimo, la proporción según las 3 categorías de riesgo de la escala PREPARE fue la siguiente [Gráfico 14]:

- Riesgo bajo (0-5 puntos): 33 pacientes $(44,6 \%)$.

- $\quad$ Riesgo medio (6-9 puntos): 28 pacientes $(37,8 \%)$.

- Riesgo alto (10-17 puntos): 13 pacientes $(17,6 \%)$. 


\begin{tabular}{|c|c|c|c|}
\hline VARIABLE & DATO & PUNTUACIÓN & $\begin{array}{l}\text { PROPORCIÓN } \\
\text { DE PACIENTES }\end{array}$ \\
\hline \multirow{2}{*}{$\begin{array}{l}\text { Albúmina } \\
\text { plasmática }\end{array}$} & $\geq 3,5 \mathrm{~g} / \mathrm{dL}$ & & \\
\hline & $<3,5 \mathrm{~g} / \mathrm{dL}$ & 5 puntos & $\begin{array}{c}23 \text { pacientes } \\
(31,1 \%)\end{array}$ \\
\hline \multirow{2}{*}{$\begin{array}{c}\text { Cirugía } \\
\text { programada }\end{array}$} & Sí & & \\
\hline & No (urgente) & 4 puntos & $\begin{array}{c}\text { Ningún paciente } \\
(0 \%)\end{array}$ \\
\hline \multirow{2}{*}{$\begin{array}{c}\text { Técnica } \\
\text { quirúrgica }\end{array}$} & Otras técnicas & & \\
\hline & $\begin{array}{l}\text { DPC o resección } \\
\text { de cabeza de } \\
\text { páncreas con } \\
\text { preservación del } \\
\text { duodeno }\end{array}$ & 2 puntos & $\begin{array}{c}74 \text { pacientes } \\
(100 \%)\end{array}$ \\
\hline \multirow{2}{*}{$\begin{array}{c}\text { Patología de } \\
\text { origen } \\
\text { pancreático }\end{array}$} & Sí & & \\
\hline & No & 2 puntos & $\begin{array}{c}31 \text { pacientes } \\
(41,9 \%)\end{array}$ \\
\hline \multirow{2}{*}{$\begin{array}{c}\text { Frecuencia } \\
\text { cardíaca }\end{array}$} & 50-80 lpm & & \\
\hline & $<50$ o $>80 \mathrm{lpm}$ & 2 puntos & $\begin{array}{c}25 \text { pacientes } \\
(33,8 \%)\end{array}$ \\
\hline \multirow{2}{*}{$\begin{array}{c}\text { Presión arterial } \\
\text { sistólica }\end{array}$} & $110-130 \mathrm{mmHg}$ & & \\
\hline & $\begin{array}{c}<110 \text { o }>130 \\
\mathrm{mmHg}\end{array}$ & 2 puntos & $\begin{array}{c}36 \text { pacientes } \\
(48,6 \%)\end{array}$ \\
\hline \multirow{2}{*}{$\begin{array}{l}\text { Hemoglobina } \\
\text { en sangre }\end{array}$} & $11,5-17 \mathrm{~g} / \mathrm{dL}$ & & \\
\hline & $<11,5$ o $>17 \mathrm{~g} / \mathrm{dL}$ & 1 punto & $\begin{array}{c}11 \text { pacientes } \\
(14,9 \%)\end{array}$ \\
\hline \multirow{2}{*}{$\begin{array}{c}\text { Riesgo } \\
\text { anestésico } \\
\text { (escala ASA) }\end{array}$} & $\leq \mathrm{II}$ & & \\
\hline & $\geq \mathrm{III}$ & 1 punto & $\begin{array}{c}28 \text { pacientes } \\
(37,8 \%)\end{array}$ \\
\hline
\end{tabular}


Gráfico 14. Gráfico de sectores que muestra la distribución de pacientes del SDPC según las categorías de riesgo de la escala PREPARE (bajo, medio y alto).

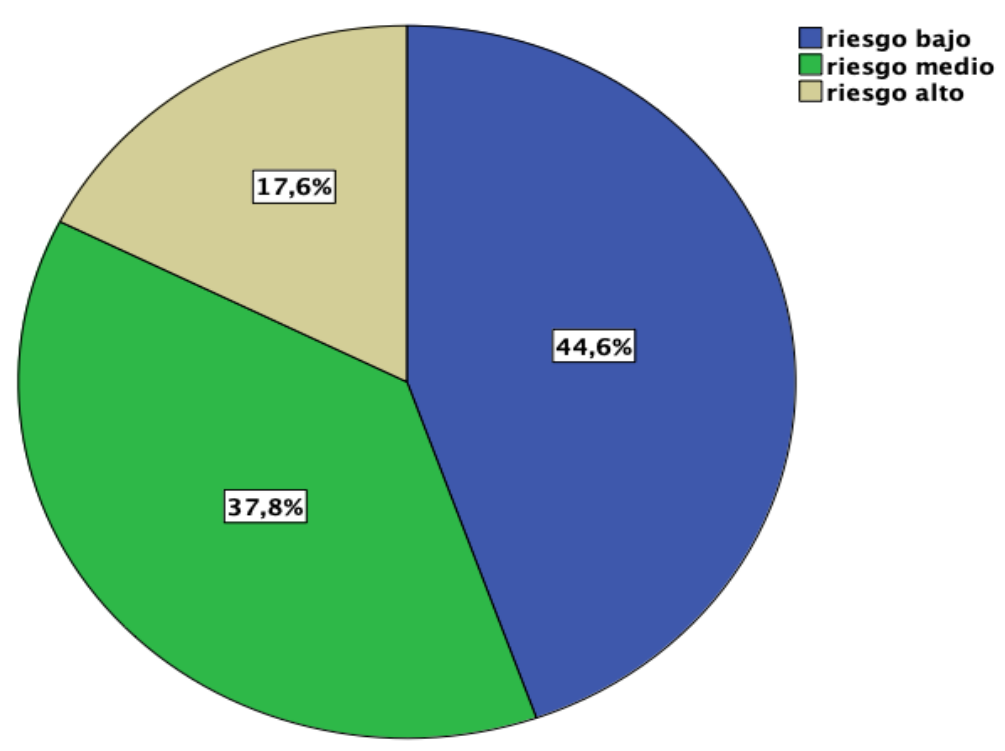

En el análisis descriptivo de las variables clínicas incluidas en la escala Wiltberger, se obtuvieron los siguientes resultados:

- Cifra de IMC: sigue una distribución normal, media de $26,3 \pm 4,3 \mathrm{Kg} / \mathrm{m}^{2}$ (rango de 17,6 a $38,8 \mathrm{Kg} / \mathrm{m}^{2}$ ).

- Grado de riesgo anestésico ASA: 46 pacientes (62,2\%) fueron grados I y II (6,8\% y 55,4\%, respectivamente), y los 28 restantes fueron ASA-III (37,8\%, ningún caso de ASA-IV).

- Enfermedad cardiovascular: 33 pacientes presentaban criterios de enfermedad cardiovascular $(44,6 \%)$.

- Enfermedad respiratoria: 14 pacientes presentaban criterios de enfermedad respiratoria $(18,9 \%)$.

La asignación de puntos en estos 74 individuos, siguiendo los criterios establecidos por la escala de Wiltberger (137) en cada una de las 4 variables, se muestra 
en la tabla 18. Asimismo, la puntuación para el SDPC siguió una distribución normal y se obtuvo una media de 5,4 \pm 4,7 puntos (rango de 0 a 16 puntos). Por ultimo, la proporción según las 3 categorías de riesgo de la escala Wiltberger fue la siguiente [Gráfico 15]:

- Riesgo bajo (0-4 puntos): 33 pacientes (44,6\%).

- Riesgo medio (5-10 puntos): 31 pacientes $(41,9 \%)$.

- Riesgo alto (11-16 puntos): 10 pacientes (13,5\%).

\begin{tabular}{|c|c|c|c|}
\hline VARIABLE & DATO & PUNTUACIÓN & $\begin{array}{l}\text { PROPORCIÓN } \\
\text { DE PACIENTES }\end{array}$ \\
\hline \multirow[t]{3}{*}{ IMC } & $<25 \mathrm{Kg} / \mathrm{m}^{2}$ & & \\
\hline & $\geq 25$ a $30 \mathrm{Kg} / \mathrm{m}^{2}$ & 1 punto & $\begin{array}{c}30 \text { pacientes } \\
(40,5 \%)\end{array}$ \\
\hline & $\geq 30 \mathrm{Kg} / \mathrm{m}^{2}$ & 4 puntos & $\begin{array}{c}15 \text { pacientes } \\
(20,3 \%)\end{array}$ \\
\hline \multirow{2}{*}{$\begin{array}{c}\text { Riesgo } \\
\text { anestésico } \\
\text { (escala ASA) }\end{array}$} & $\leq \mathrm{II}$ & & \\
\hline & $\geq \mathrm{III}$ & 2 puntos & $\begin{array}{c}28 \text { pacientes } \\
(37,8 \%)\end{array}$ \\
\hline \multirow{2}{*}{$\begin{array}{l}\text { Enfermedad } \\
\text { cardiovascular }\end{array}$} & No & & \\
\hline & Sí & 6 puntos & $\begin{array}{c}33 \text { pacientes } \\
(44,6 \%)\end{array}$ \\
\hline \multirow{2}{*}{$\begin{array}{l}\text { Enfermedad } \\
\text { respiratoria }\end{array}$} & No & & \\
\hline & Sí & 4 puntos & $\begin{array}{c}14 \text { pacientes } \\
(18,9 \%)\end{array}$ \\
\hline
\end{tabular}


Gráfico 15. Gráfico de sectores que muestra la distribución de pacientes del SDPC según las categorías de riesgo de la escala Wiltberger (bajo, medio y alto).

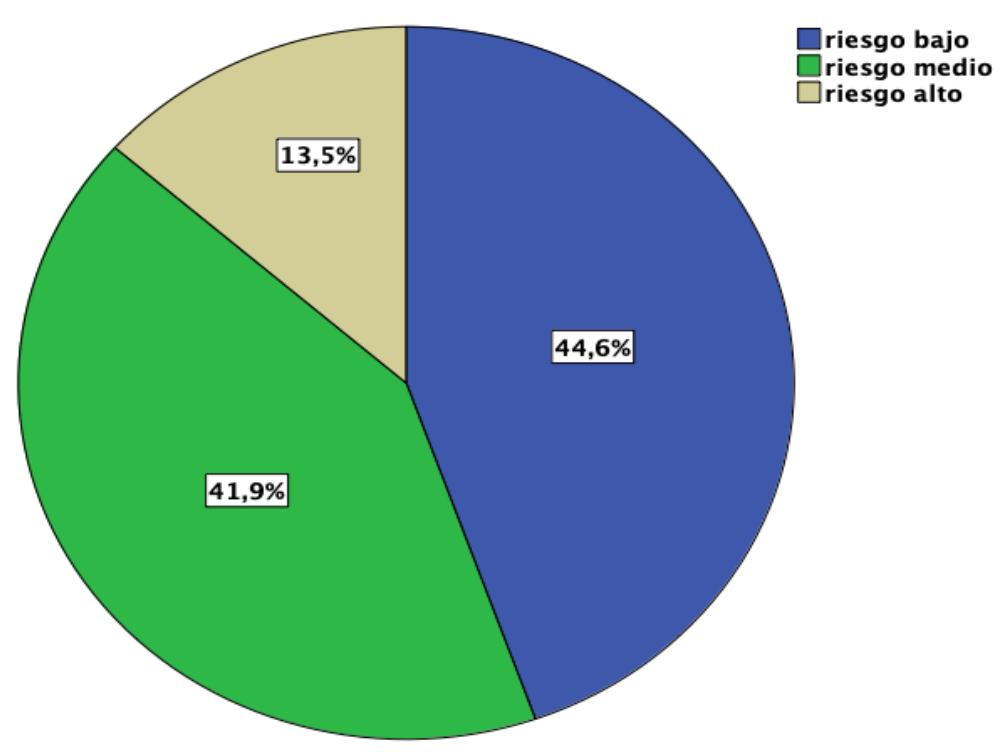

\subsubsection{3.- VARIABLES INTRAOPERATORIAS}

En la totalidad de los casos se utilizó abordaje laparotómico, el 11\% necesitaron trasfusión de concentrado de hematíes. En el 8,1\% de los pacientes, se utilizó la reconstrucción en Y de Roux. El 91,9\% de los pacientes fue reconstruido según la técnica de Child y de ellos en el 8,1\% fue asociada una anastomosis de Braun. En 64 pacientes $(86,5 \%)$ se utilizó drenaje intraabdominal. 


\subsubsection{4.- VARIABLES POSTOPERATORIAS}

Como ya se mencionó en los resultados de la SG, todos los enfermos acudieron a la unidad de reanimación postquirúrgica después de la resección pancreática. La estancia postoperatoria en dicha unidad siguió una distribución no normal y tuvo una mediana de 3 días y un RIQ de 2-4 días.

Todos los pacientes menos uno, consiguieron tolerancia oral durante el postoperatorio, se obtuvo una distribución no normal con una mediana de 4 días y un RIQ de 3-7 días. En cuanto al uso de NPT, en el 93,2\% de los pacientes fue necesaria después de la cirugía. La distribución de la duración de días de NPT en estos pacientes fue no normal con una mediana de 7 días con un RIQ de 6-11 días.

El 78,4\% de los pacientes tuvo algún tipo de complicación en el postoperatorio. La distribución de estas complicaciones según la gravedad en base a clasificación de Dindo et al. (114) se muestra en el gráfico 16. A su vez el 32,4\% de los enfermos tuvieron complicaciones graves (Clavien $\geq$ IIIa, definido como complicación grave según Uzunoglu et al.) (136). El 20,3\% de los pacientes tuvieron complicaciones graves (Clavien $\geq$ IIIb, definido como complicación grave según Wiltberger el al.) (137). 
Gráfico 16. Gráfico de barras que muestra la distribución de complicación según los grados de gravedad de Dindo et al. (114).

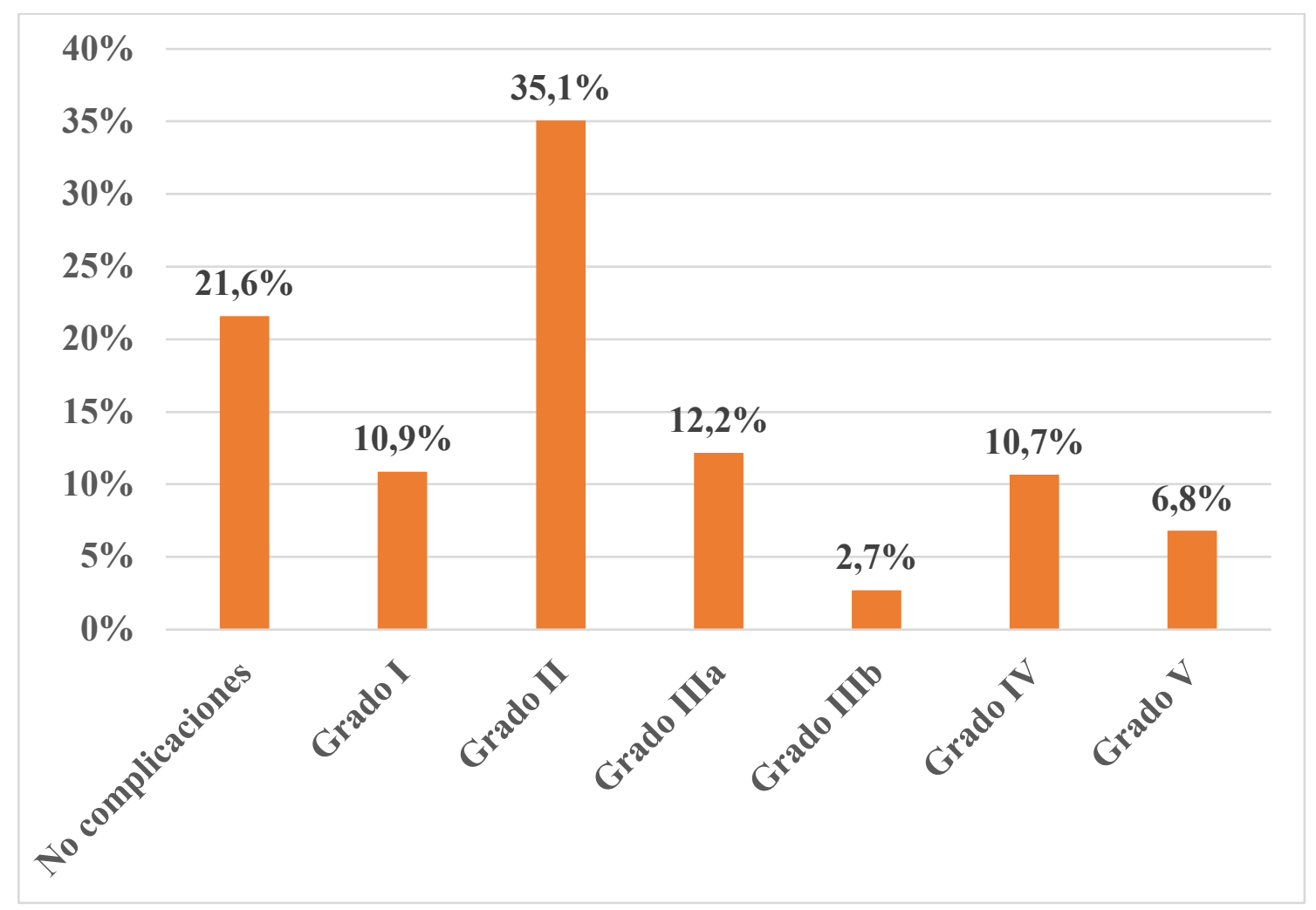

Las complicaciones específicas que acontecieron tras pancreatectomía fueron las siguientes:

- El 27\% de los enfermos desarrollaron FPPO, El 13,5\% desarrollaron FPCR. El gráfico 17 muestra la distribución según los grados de gravedad del ISGPS (115).

- El 33,8\% de los pacientes desarrollaron RVG, la distribución según los grados de gravedad del ISGPS (121) puede observarse en el gráfico 18.

- El 23\% de los pacientes desarrollaron HPP, la distribución según los grados de gravedad del ISGPS (128) puede observarse en el gráfico 19. 
Gráfico 17. Gráfico de barras que muestra la distribución según los grados de gravedad de FPPO (115).

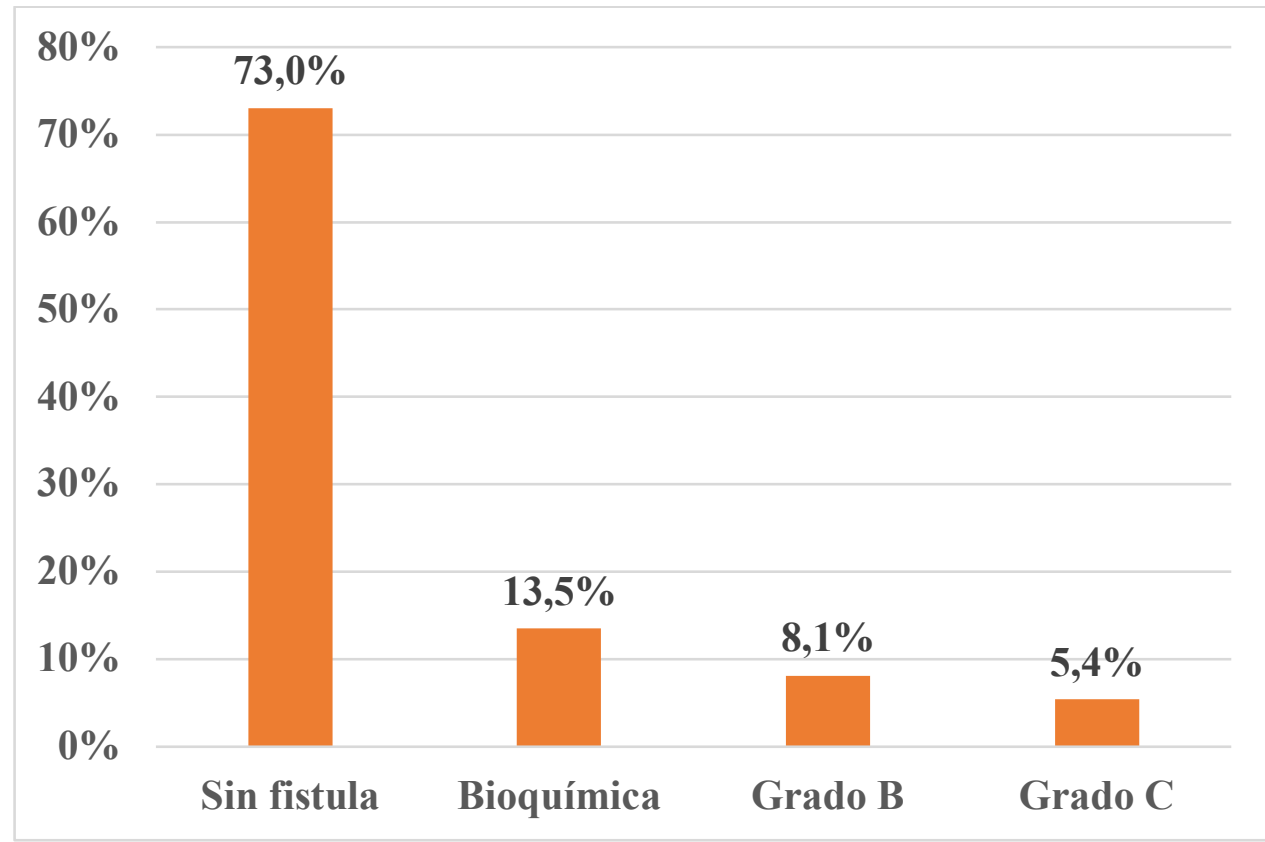

Gráfico 18. Gráfico de barras que muestra la distribución según los grados de gravedad de RVG (121).

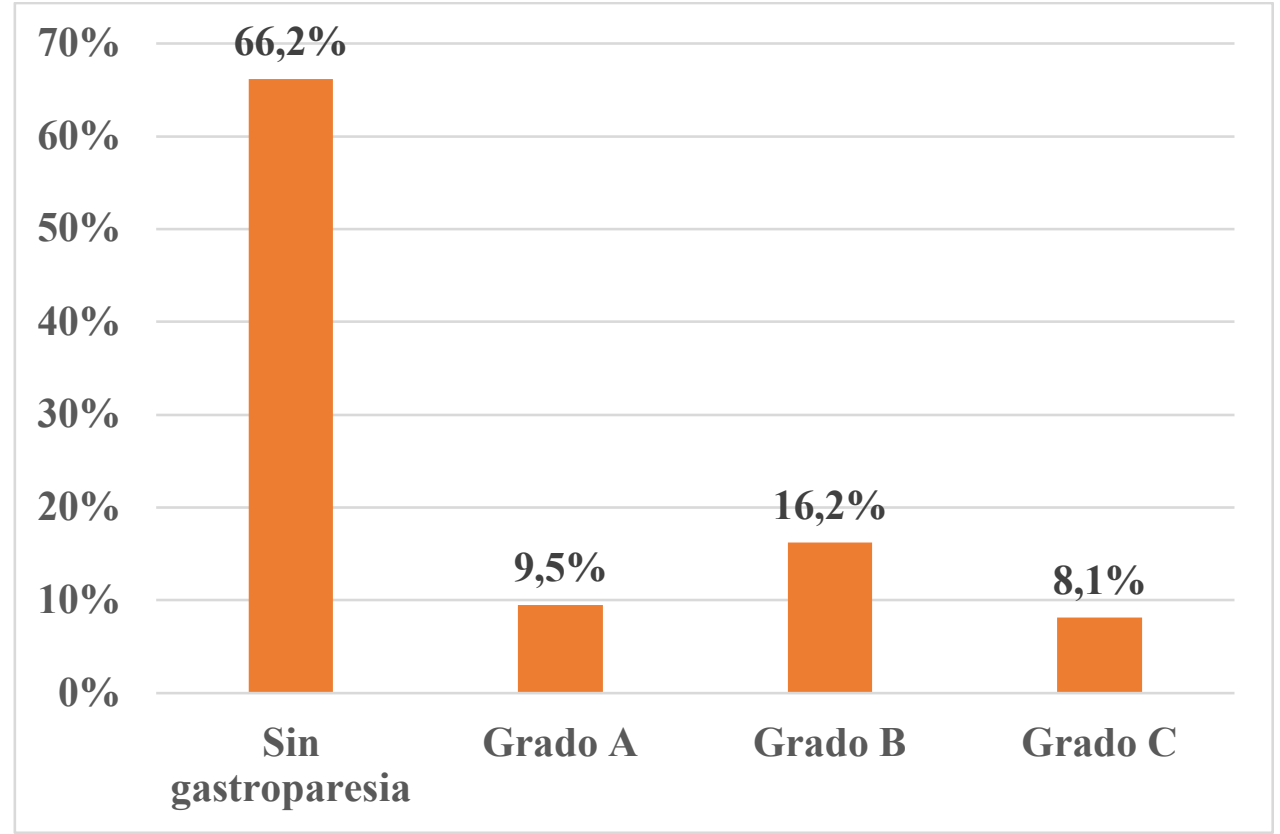


Gráfico 19. Gráfico de barras que muestra la distribución según los grados de gravedad de HPP (128).

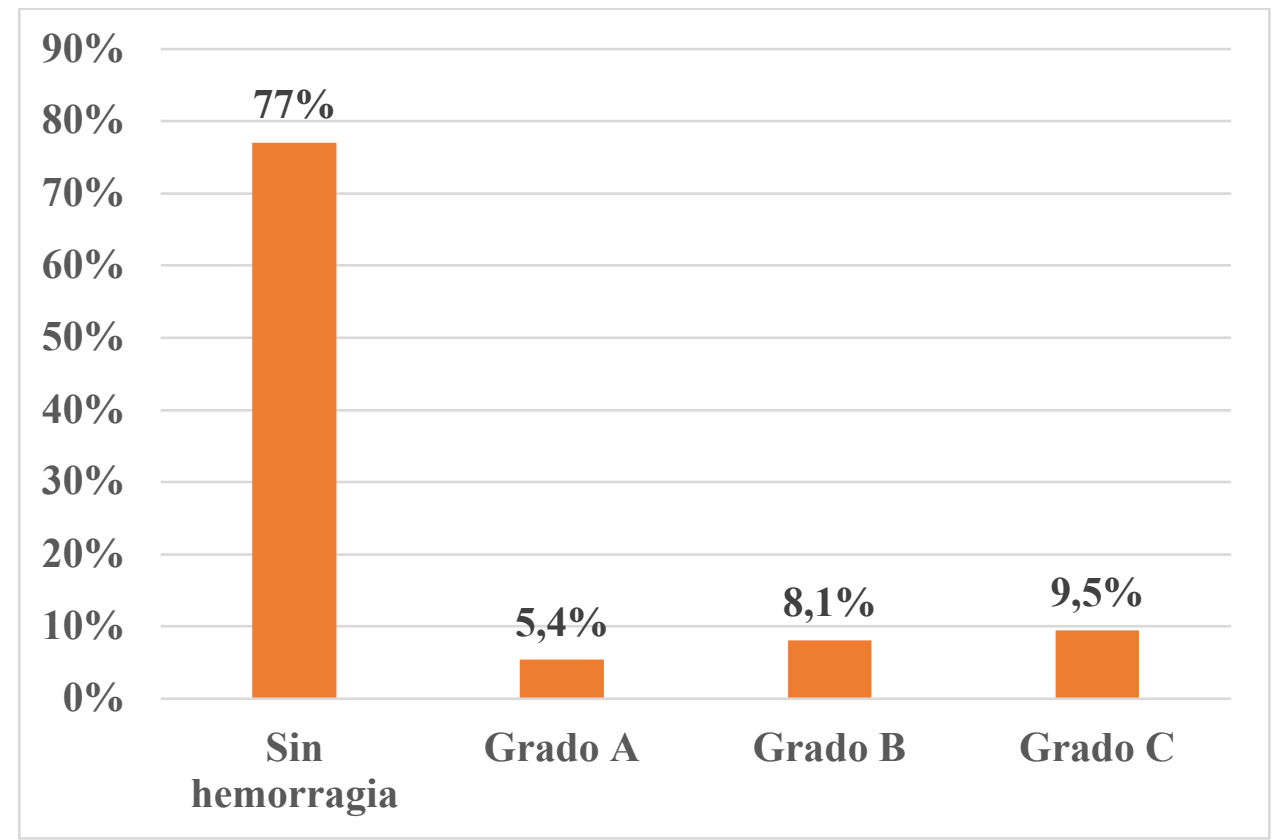

La IHQ aconteció en el 21,6\% de los pacientes. En el 10,8\% de los pacientes fue necesaria la reintervención quirúrgica, de los cuales, la causa de la misma ya se expuso en el gráfico 9. Las reintervenciones del SDPC coinciden con los de la SG dado que todas ellas corresponden a pacientes a los que se les había realizado DPC.

La estancia postoperatoria global siguió una distribución no normal con una mediana de 11 días y un RIQ de 9-15 días. 


\subsection{2.- ANÁLISIS INFERENCIAL}

Tras establecer la proporción de individuos con complicaciones graves $\geq$ IIIa en este SDPC para cada una de las categorías de riesgo de la escala PREPARE (136), se obtiene la siguiente distribución que mostró diferencias estadísticamente significativas $(\mathrm{p}=0,01)$. [gráfico 20]:

- Riesgo bajo: 8 pacientes con complicaciones graves sobre $33(24,2 \%)$.

- Riesgo medio: 7 pacientes con complicaciones graves sobre $28(25 \%)$.

- Riesgo alto: 9 pacientes con complicaciones graves sobre $13(69,2 \%)$.

Gráfico 20: Gráfico de barras que muestra la proporción de pacientes con complicaciones graves (Clavien $\geq$ IIIa) en cada una de las categorías de la escala PREPARE en el análisis del SDPC de pacientes.

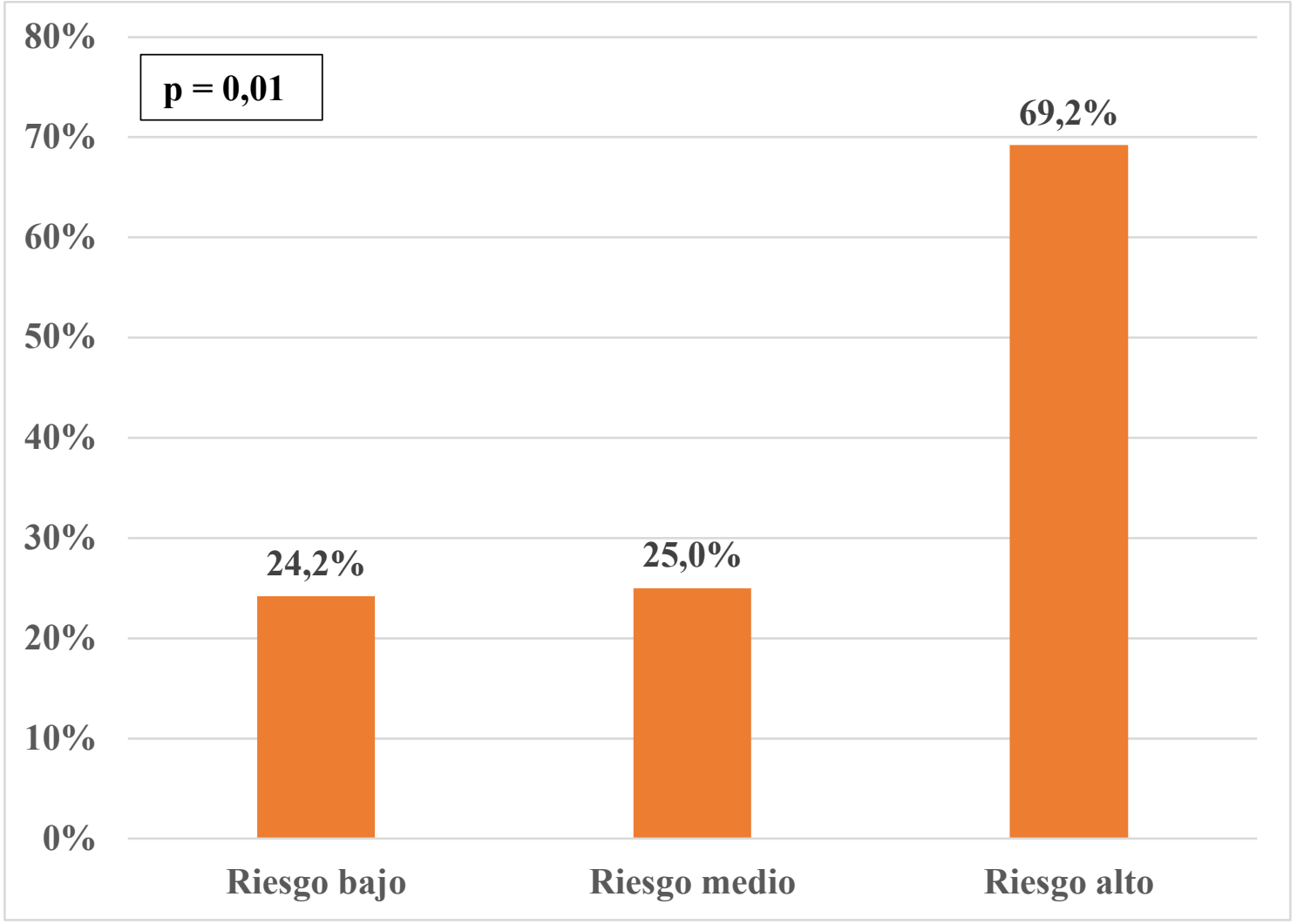


Para comprobar estadísticamente la fiabilidad de este índice, se notificó un $\mathrm{AUC}=0,65(\mathrm{IC} 95 \%=0,508-0,793, \mathrm{p}=0,037)$ en la curva $R O C$ [Gráfico 21]. A la luz de los datos anteriormente expuestos, en el análisis del SDPC, los pacientes catalogados como de alto riesgo según la escala PREPARE (136) presentan 2,34 veces más probabilidad de sufrir morbilidad grave respecto a los pacientes de la categoría de bajo riesgo $(\mathrm{RR}=2,34$, IC 95\% $=1,18-4,67)$. Tras aplicar el test de Hosmer-Lemeshow, se obtuvo un porcentaje pronosticado correcto del 74,3\% y una $\mathrm{p}=0,095$

Gráfico 21.: Curva $R O C$ para evaluar la fiabilidad de la escala PREPARE en el SDPC.

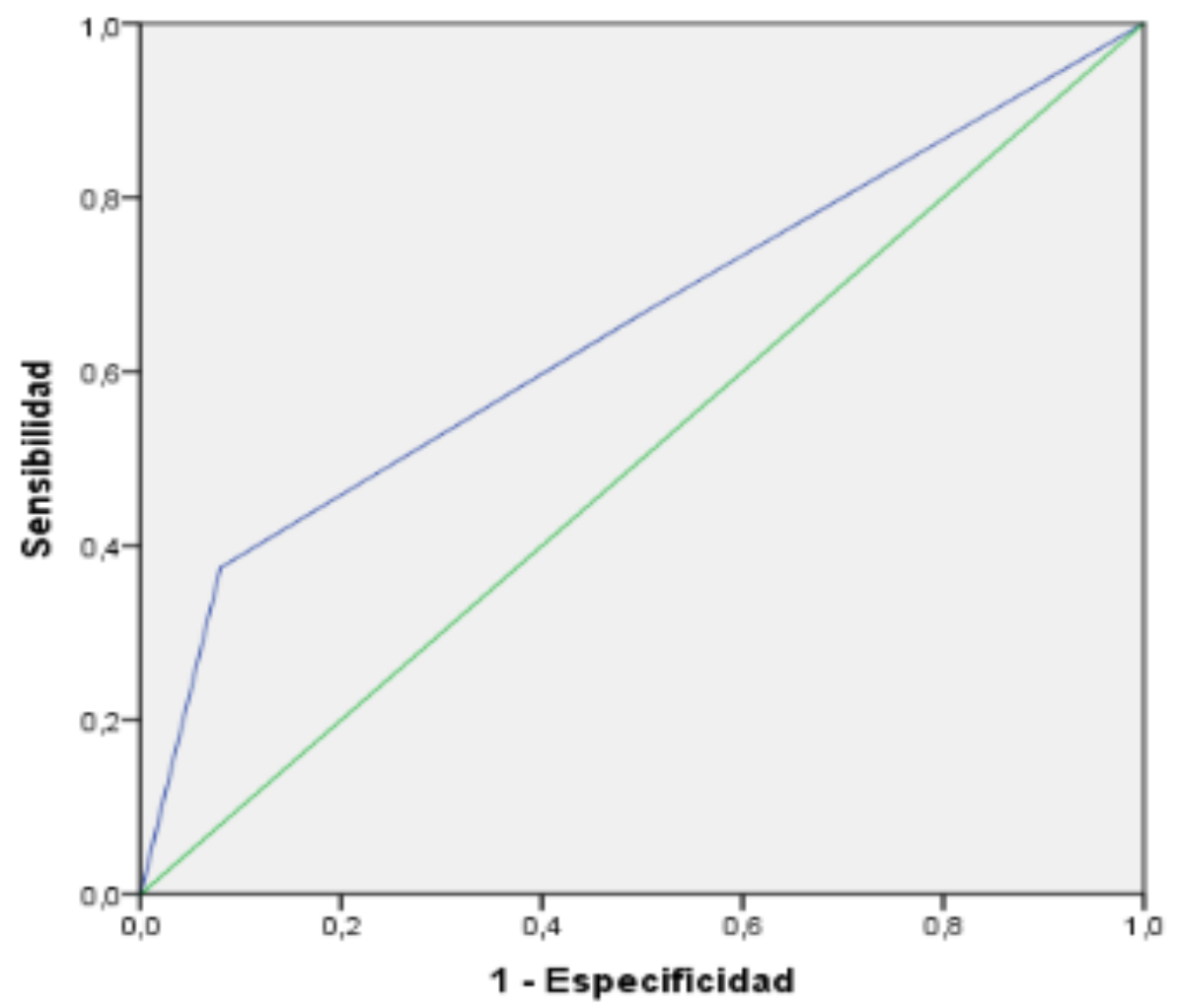

Tras establecer la proporción de individuos con complicaciones graves $\geq$ IIIb en este SDPC para cada una de las categorías de riesgo de la escala Wiltberger (137), se 
obtiene la siguiente distribución que mostró diferencias estadísticamente significativas ( $\mathrm{p}$ $=0,006)$. [gráfico 22]:

- Riesgo bajo: 2 pacientes con complicaciones graves sobre $33(6,1 \%)$.

- Riesgo medio: 8 pacientes con complicaciones graves sobre $31(25,8 \%)$.

- Riesgo alto: 5 pacientes con complicaciones graves sobre $10(50 \%)$.

Gráfico 22: Gráfico de barras que muestra la proporción de pacientes con complicaciones graves (Clavien $\geq \mathrm{IIIb}$ ) en cada una de las categorías de la escala Wiltberger en el análisis del SDPC de pacientes.

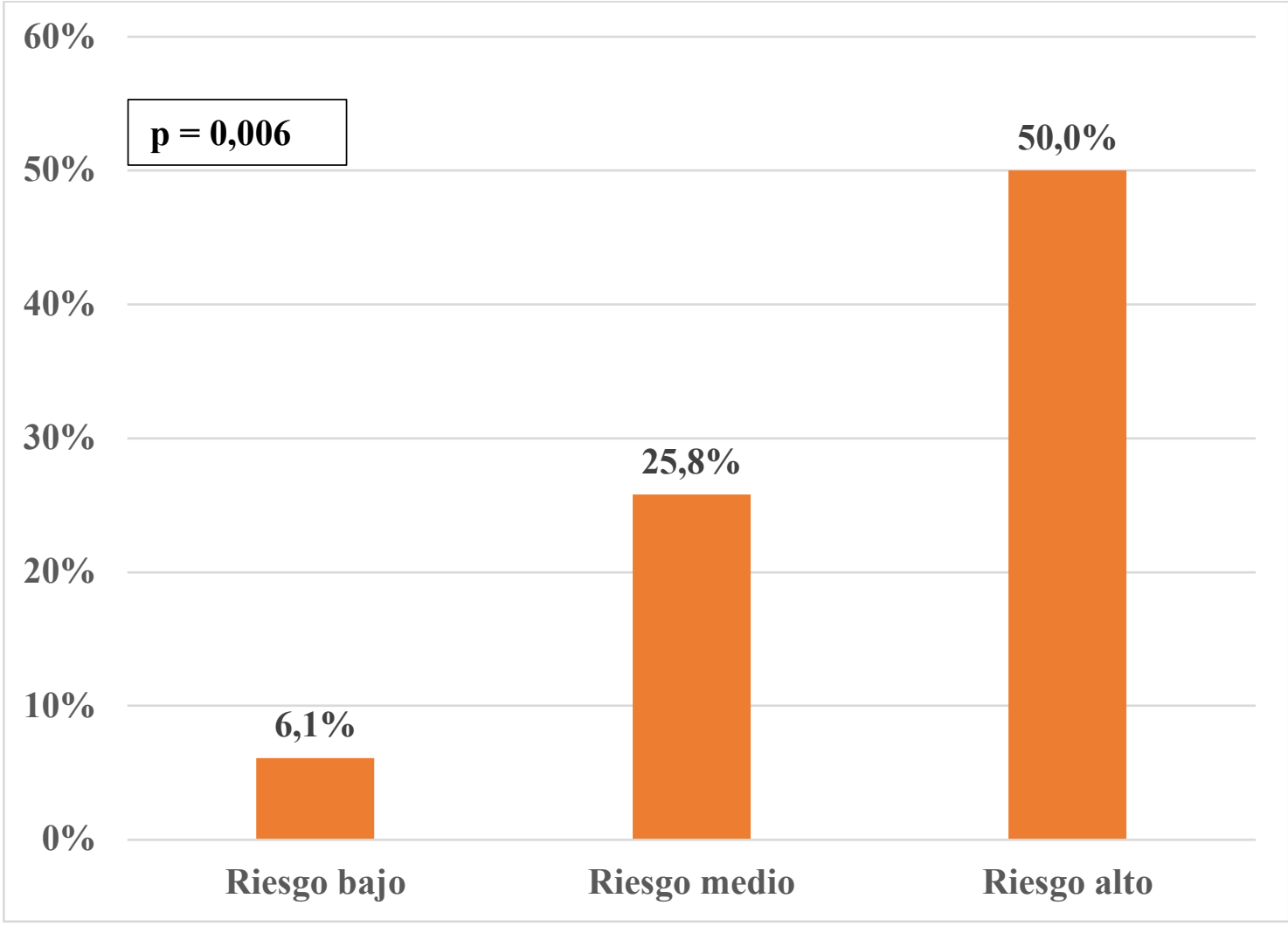

Para comprobar estadísticamente la fiabilidad de este índice, se notificó un AUC $=0,738(\mathrm{IC} 95 \%=0,6-0,876, \mathrm{p}=0,005)$ en la curva $R O C$ [Gráfico 23]. A la luz de los datos anteriormente expuestos, en el análisis del SDPC, los pacientes catalogados como 
de alto riesgo según la escala Wiltberger (137) presentan casi 4 veces más probabilidad de sufrir morbilidad grave respecto a los pacientes de la categoría de bajo riesgo $(\mathrm{RR}=$ 3,87, IC 95\% = 1,57 - 9,55). Tras aplicar el test de Hosmer-Lemeshow, se obtuvo un porcentaje pronosticado correcto del 79,7\% y una $\mathrm{p}=0,619$.

Gráfico 23.: Curva ROC para evaluar la fiabilidad de la escala Wiltberger en el SDPC.

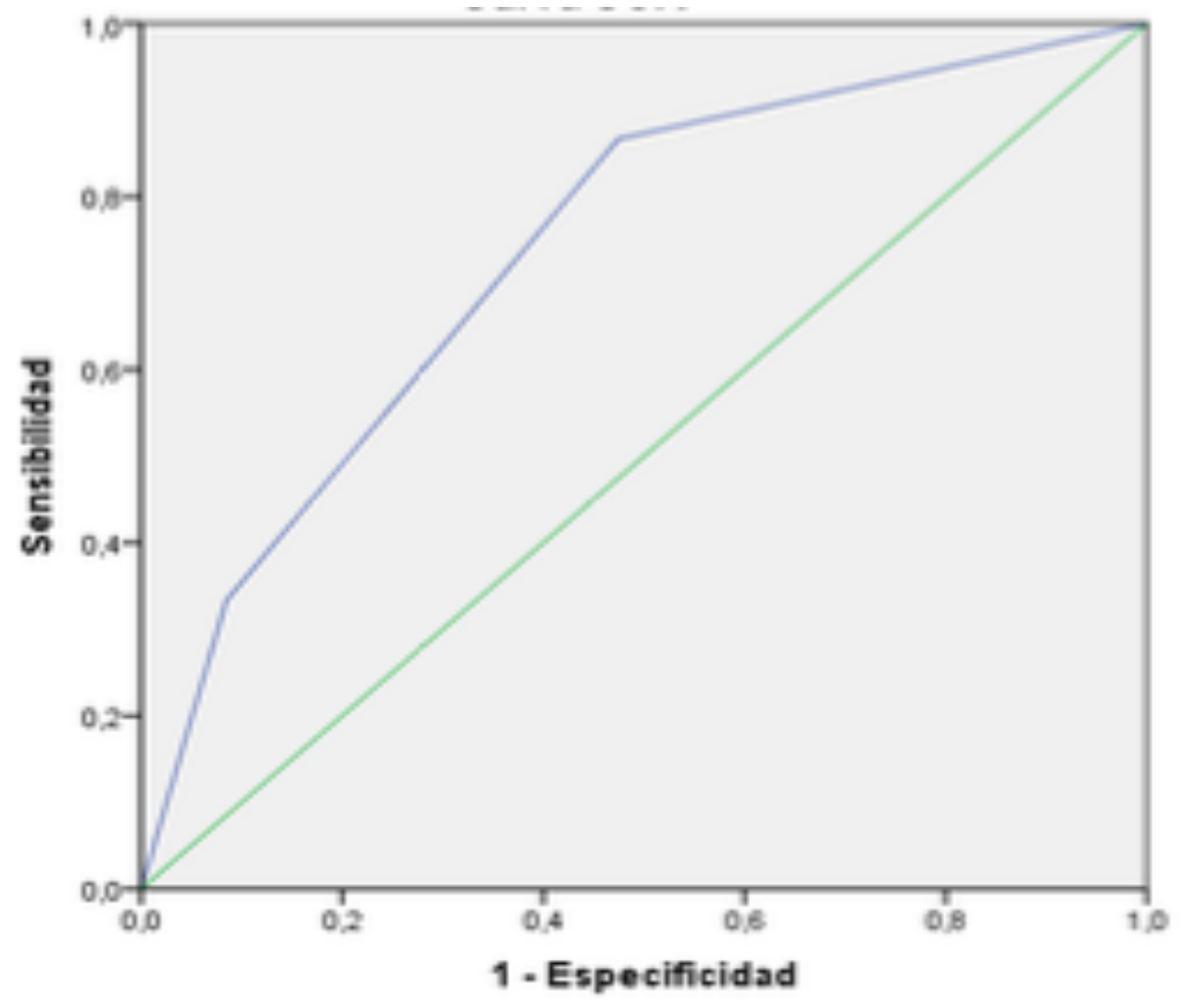

A continuación, en la tabla 19 se describe el análisis bivariante entre complicaciones globales, reintervención, FPPO, RVG, HPP e IHQ y los grados de riesgo de la escala PREPARE y Wiltberger. 


\begin{tabular}{|c|c|c|c|c|c|c|c|}
\hline \multicolumn{8}{|c|}{$\begin{array}{l}\text { Tabla 19: Análisis bivariante en el SDPC (los valores entre paréntesis representan } \\
\text { porcentajes). }\end{array}$} \\
\hline & & \multicolumn{3}{|c|}{ Grados PREPARE } & \multicolumn{3}{|c|}{ Grados Wiltberger } \\
\hline & & Bajo & Medio & Alto & Bajo & Medio & Alto \\
\hline \multirow[b]{2}{*}{$\begin{array}{c}\text { Complicaciones } \\
\text { globales }\end{array}$} & No & $9(27,3)$ & $7(25)$ & $0(0)$ & $8(24,2)$ & $8(25,8)$ & $0(0)$ \\
\hline & Sí & $24(72,7)$ & $21(75)$ & $13(100)$ & $25(75,8)$ & $23(74,2)$ & $10(100)$ \\
\hline \multicolumn{2}{|c|}{ P-VALOR } & \multicolumn{3}{|c|}{$\underline{0,029}$} & \multicolumn{3}{|c|}{ N.S. } \\
\hline \multirow[b]{2}{*}{ Exitus } & No & $33(100)$ & $25(89,3)$ & $11(84,6)$ & $32(97)$ & $28(90,3)$ & $9(90)$ \\
\hline & Sí & $0(0)$ & $3(10,7)$ & $2(15,4)$ & $1(3)$ & $3(9,7)$ & $1(10)$ \\
\hline \multicolumn{2}{|c|}{ P-VALOR } & \multicolumn{3}{|c|}{$\underline{0,041}$} & \multicolumn{3}{|c|}{ N.S. } \\
\hline \multirow[b]{2}{*}{ Reintervención } & No & $33(100)$ & $25(89,3)$ & $9(69,2)$ & $33(100)$ & $26(83,9)$ & $8(80)$ \\
\hline & Sí & $0(0)$ & $3(10,7)$ & $4(30,8)$ & $0(0)$ & $5(16,1)$ & $2(20)$ \\
\hline \multicolumn{2}{|c|}{ P-VALOR } & \multicolumn{3}{|c|}{$\underline{0,004}$} & \multicolumn{3}{|c|}{$\underline{0,012}$} \\
\hline \multirow{4}{*}{ FPPO } & No & $23(69,7)$ & $22(78,6)$ & $9(69,2)$ & $27(81,8)$ & $21(67,7)$ & $6(60)$ \\
\hline & Bioquímica & $6(18,2)$ & $2(7,1)$ & $2(15,4)$ & $4(12,1)$ & $6(19,4)$ & $0(0)$ \\
\hline & $\mathrm{B}$ & $4(12,1)$ & $2(7,1)$ & $0(0)$ & $2(6,1)$ & $1(3,2)$ & $3(30)$ \\
\hline & $\mathrm{C}$ & $0(0)$ & $2(7,1)$ & $2(15,4)$ & $0(0)$ & $3(9,7)$ & $1(10)$ \\
\hline \multicolumn{2}{|c|}{ P-VALOR } & \multicolumn{3}{|c|}{ N.S. } & \multicolumn{3}{|c|}{$\underline{\mathbf{0 , 0 3 3}}$} \\
\hline \multirow{4}{*}{ RVG } & No & $24(72,7)$ & $19(67,9)$ & $6(46,2)$ & $26(78,8)$ & $18(58,1)$ & $5(50)$ \\
\hline & $\bar{A}$ & $2(6,1)$ & $3(10,7)$ & $2(15,4)$ & $2(6,1)$ & $5(16,1)$ & $0(0)$ \\
\hline & B & $4(12,1)$ & $5(17,9)$ & $3(23,1)$ & $5(15,2)$ & $5(16,1)$ & $2(20)$ \\
\hline & $\mathrm{C}$ & $3(9,1)$ & $1(3,6)$ & $2(15,4)$ & $0(0)$ & $3(9,7)$ & $3(30)$ \\
\hline \multicolumn{2}{|c|}{ P-VALOR } & \multicolumn{3}{|c|}{ N.S. } & \multicolumn{3}{|c|}{$\underline{\mathbf{0 , 0 2 9}}$} \\
\hline \multirow{4}{*}{ HPP } & No & $25(75,8)$ & $24(85,7)$ & $8(61,5)$ & $29(87,9)$ & $23(74,2)$ & $5(50)$ \\
\hline & A & $3(9,1)$ & $1(3,6)$ & $0(0)$ & $3(9,1)$ & $1(3,2)$ & $0(0)$ \\
\hline & B & $4(12,1)$ & $1(3,6)$ & $1(7,7)$ & $1(3)$ & $3(9,7)$ & $2(20)$ \\
\hline & $\mathrm{C}$ & $1(3)$ & $2(7,1)$ & $4(30,8)$ & $0(0)$ & $4(12,9)$ & $3(30)$ \\
\hline \multicolumn{2}{|c|}{ P-VALOR } & \multicolumn{3}{|c|}{ N.S. } & \multicolumn{3}{|c|}{$\underline{\mathbf{0 , 0 1 5}}$} \\
\hline \multirow[b]{2}{*}{ IHQ } & No & $28(84,8)$ & $20(71,4)$ & $10(76,9)$ & $29(87,9)$ & $23(74,2)$ & $6(60)$ \\
\hline & Sí & $5(15,2)$ & $8(28,6)$ & $3(23,1)$ & $4(12,1)$ & $8(25,8)$ & $4(40)$ \\
\hline \multicolumn{2}{|c|}{ P-VALOR } & \multicolumn{3}{|c|}{ N.S. } & & N.S. & \\
\hline
\end{tabular}




\section{DISCUSIÓN}


Francisco Javier Tejero Pintor 


\section{DISCUSIÓN}

La cirugía pancreática es un procedimiento considerado como complejo por lo que tiene un riesgo alto de complicaciones postoperatorias. La mejoría de las técnicas quirúrgicas y la estandarización en el manejo perioperatorio global del paciente, así como la experiencia acumulada en centros de alto volumen, ha reducido las complicaciones postoperatorias en este tipo de intervención. La morbilidad grave, normalmente definida por la mayoría de cirujanos según la escala de Dindo-Clavien (114) con los grados III, IV y V, supone casi un tercio en el postoperatorio de este tipo de cirugía con un 30\%, tal y como notifican algunos estudios multicéntricos $(136,142)$. La aparición de estas complicaciones durante el postoperatorio, supone un incremento cuantioso del gasto sanitario por proceso, derivado de la realización de pruebas diagnósticas, prolongación del ingreso, necesidad de reintervención, etc. (143), además, del riesgo potencial para la vida del paciente.

En los últimos años, se han publicado artículos que clasifican y cuantifican complicaciones post-pancreatectomía, tales como el retraso del vaciamiento gástrico (RVG), fístula pancreática postoperatoria (FPPO) o la hemorragia postoperatoria postpancreatectomía (HPP) $(115,121,128)$. Por su parte, otros trabajos han estudiado los factores de riesgo asociados a su aparición, estableciendo escalas cuyo objetivo es estimar la contingencia que presenta el paciente de sufrir eventos adversos en el postoperatorio de una resección pancreática. Resultan especialmente útiles aquellos que se basan en variables clínico-analíticas preoperatorias y que se desarrollaron específicamente con pacientes sometidos a este tipo de cirugía: la escala PREPARE (136), Wiltberger (137), 
el de Parikh et al. (144), el de Greenblatt et al. (133), el WHipple-ABACUS (132), o el de Aoki et al (129).

Por otra parte, sólo dos de estos seis índices pueden emplearse en todo tipo de técnicas de la cirugía pancreática, a saber: la escala PREPARE (136) y el propuesto por Parikh (144). Precisamente, el tipo de técnica de resección pancreática sí es una variable incluida en ambos. Las tres restantes escalas [el del Wiltberger (137), el de Greenblatt et al. (133) y el WHipple-ABACUS (132)] fueron diseñados exclusivamente para la DPC, al igual que el de Braga et al. (130), aunque éste último se basa, casi en su totalidad, en datos clínicos intraoperatorios, a diferencia de los otros seis índices citados.

En el trabajo que aquí presentamos se ha llevado a cabo un estudio observacional prospectivo en el que se compara la escala PREPARE (136) y Wiltberger (137) como herramientas capaces de predecir morbimortalidad postoperatoria tras cirugía pancreática mayor. La recogida de datos prospectiva, presenta la ventaja evidente respecto a una recogida retrospectiva de información clínica, de minimizar la pérdida de datos y evita la heterogeneidad de los mismos. Así pues, se puede confirmar que no se ha detectado pérdida de datos para las variables analizadas en este estudio y por tanto no existe sesgo alguno, en este sentido. Asimismo, el diseño del trabajo como estudio de cohortes, aporta rigor científico, estando catalogado con un nivel de calidad medio-alto, inmediatamente por debajo de los ensayos clínicos aleatorizados, según la versión más reciente propuesta de la llamada "Medicina basada en la evidencia" (145).

Para realizar esta comparación ha sido necesario establecer un subgrupo de pacientes tratados mediante la técnica de duodenopancreatectomía cefálica (DPC), dado 
que para ello fue descrita la escala de Wiltberger (137). Así pues, hemos obtenido datos sobre la escala PREPARE en la serie global (SG) y en el subgrupo DPC (SDPC), y sobre la escala Wiltberger en este mismo subgrupo.

\section{1.- SERIE GLOBAL}

A lo largo de 53 meses de estudio se completaron 97 resecciones pancreáticas mayores. Este tamaño muestral ha permitido realizar test estadísticos avanzados para comprobar la fiabilidad de la escala PREPARE (136). El criterio de exclusión aplicado elimina del registro en la base de datos a aquellos individuos en quienes se abortó la resección pancreática por progresión e irresecabilidad tumoral y, por tanto, no tendría sentido evaluar las escalas. Esta contingencia ocurre en hasta un 20-30\% de pacientes con tumores malignos del páncreas y de la llamada región periampular considerados como extirpables en la evaluación preoperatoria (146). Otros criterios de exclusión establecidos fueron la cirugía pancreática asociada a otro procedimiento quirúrgico ya que este puede aumentar la morbilidad, las enucleaciones y las pancreatectomías realizadas por otra unidad diferente a hepatobiliopancreática. Evaluando nuevamente el tamaño muestral, la cifra de 97 pacientes es escasa en comparación con el volumen de enfermos de los grandes centros de referencia europeos para cirugía pancreática, como son los que participaron en la descripción de la escala PREPARE (136). De igual modo, el análisis de los 97 pacientes supone un $80 \%$ de los casos incluidos en el trabajo de Celik et al. (139) ( $\mathrm{n}=122)$, realizado en Turquía entre 2010 y 2015, que como ya se indicó en el epígrafe de Justificación, es la primera publicación en que se analiza la escala PREPARE, desde que éste fue publicado en 2014. Sin embargo, la ratio de pacientes intervenidos es incluso mayor en nuestra Unidad: 97 procedimientos pancreáticos en 53 meses suponen 1,8 al 
mes, y 122 en 72 meses, suponen 1,7 al mes, en el caso de grupo turco. Además, nuestro estudio presenta la ventaja, sobre el trabajo de Celik et al. (139), de ser prospectivo.

\subsection{1.- ANÁLISIS DE LOS RESULTADOS DESCRIPTIVOS}

En la SG, el 50,5\% de la muestra fueron hombres En los trabajos que analizaron escalas predictoras de morbimortalidad postoperatoria tras resección pancreática mayor, esta cifra oscila entre $51 \%$ y $62 \%(129,130,132-137,139)$ [Gráfico 24]. Específicamente en los trabajos de Uzunoglu et al. (136) y Celik et al. (139) donde ambos utilizan la escala PREPARE, el porcentaje de hombres es ligeramente superior siendo 55,8\% y 58,2\% respectivamente.

Gráfico 24.: Comparación de nuestros resultados con la literatura en cuanto a la distribución por sexo de pacientes sometidos a resección pancreática mayor $(129,130,132-137,139)$.

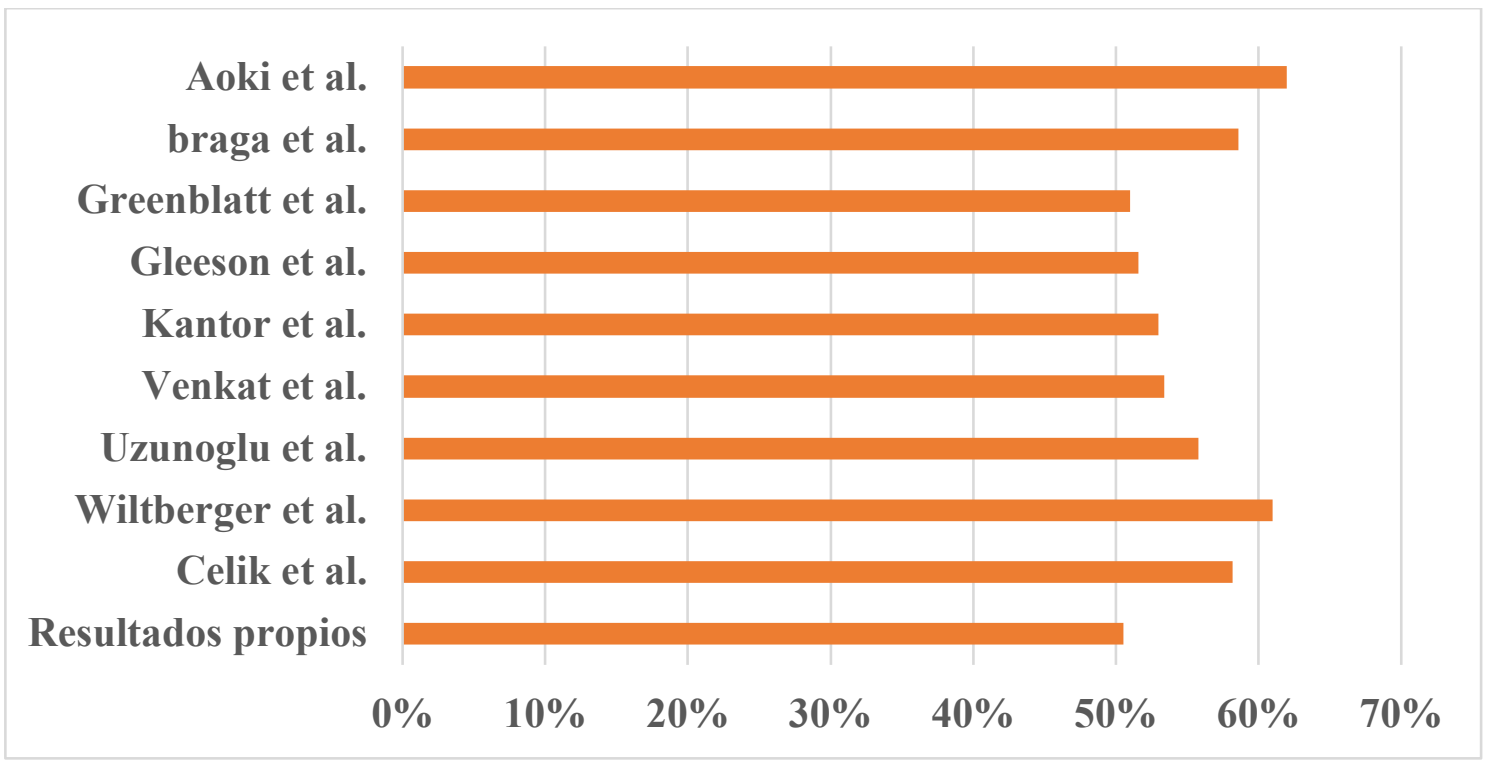


En estas mismas series de pacientes con tumores pancreáticos o de la encrucijada pancreatobiliar, las edades medias, son similares a lo que se ha descrito en este trabajo. La edad media de nuestros pacientes en la SG en el momento de la intervención fue de $61 \pm 11$ años, lo que se asemeja a publicaciones similares de otros autores, donde la edad de intervención se sitúa entre los 58 y los 69 años $(129,130,132-135,137,139)$.

En cuanto a la comorbilidad asociada en estos pacientes, destacamos que casi una cuarta parte de ellos consumía alcohol y tabaco (23,7\% y $24,7 \%$ respectivamente). Otros factores de riesgo cardiovasculares fueron la dislipemia 30,9\%, la diabetes $22,7 \%$, y la hipertensión arterial que fue la comorbilidad más prevalente en esta serie de pacientes y estuvo presente en el $36,1 \%$ de ellos.

En comparación con los datos de la literatura revisada, se puede apreciar una clara diferencia en cuanto a la proporción de pacientes que consumían alcohol. Según Greenblatt et al. (133), únicamente el 3,5\% de los enfermos a los que se les había realizado una resección pancreática lo consumían. Aunque no definen exactamente la cantidad de alcohol ingerido necesario que se requería para catalogarse como positivo en su consumo, simplemente hacen referencia a que la toma de más de 2 bebidas al día, sin especificar gramos de etanol, se consideraba consumo de alcohol. En nuestro trabajo, esta variable ha sido definida más detalladamente, tal y como se ha explicado en el apartado de pacientes y métodos, el consumo de alcohol se ha definido como una bebida estándar o 10 gramos de alcohol a la semana, equivalente a una caña de cerveza 25 centilitros o un vaso de vino 10 centilitros. Esta diferencia metodológica entre el presente estudio y el de Greenblatt et al. (133) es la razón de esta marcada desproporción. 
Según Gleeson et al. (132) el 36,5\% de los enfermos de su serie eran fumadores, que constituye 12 puntos porcentuales más que en nuestro trabajo. El resto de comorbilidades están en consonancia con lo publicado en otras grandes series: la diabetes oscila entre el 23 y $33,8 \%$ de los pacientes $(129,130,133,134,137)$ y la hipertensión arterial entre el $34,6 \%$ y el $53 \%(129,132-134)$

En cuanto al uso de prótesis biliar preoperatoria, en el 63,9\% de los pacientes fue colocada a criterio del endoscopista. Analizando únicamente a los pacientes que fueron sometidos a DPC y duodenopancreatectomía total (DPT) esta cifra asciende al 78,5\%. En tan solo una de las anteriores series descritas analizan el porcentaje de pacientes en el que fue necesaria esta prótesis biliar. Según Kantor et al. (134) solo el 27,8\% de los pacientes a los que se les realizó DPC fue necesario. Un metanálisis publicado en el año 2017 por Scheufele et al. (147) concluyó que el uso rutinario de prótesis biliar preoperatoria aumenta las complicaciones postoperatorias y la infección de herida quirúrgica (IHQ), por lo que en pacientes que no han tenido criterios de sepsis biliar, la cirugía deberá realizarse sin utilizar prótesis biliar preoperatoria.

En la tabla 20 se muestra una comparativa de la proporción de enfermos según la distribución por categorías de todas las variables de la escala PREPARE en los datos propios de este trabajo en la SG junto con lo publicado por Uzunoglu et al. (136) y Celik et al. (139). En dicha tabla, las diferencias más notables que se pueden apreciar son:

- Los pacientes incluidos en este estudio, parecen estar peor nutridos, ya que la proporción de individuos con albúmina $<3,5$ gramos por decilitro $(\mathrm{g} / \mathrm{dL})$ es mayor respecto a la de Uzunoglu et al. (136). 
- Cabe destacar que en la SG de este análisis no se ha realizado ninguna intervención quirúrgica urgente. Sin embargo, en los datos aportados por Uzunoglu et al. (136) y Celik et al. (139) la resección pancreática con carácter urgente fue realizada hasta en 1 de cada 15 enfermos en el caso de éste último.

- La distribución de los tipos de resección pancreática es muy similar entre los tres trabajos.

- En nuestro trabajo se objetivó menor patología de origen pancreático asociada a la resección de páncreas que en los otros dos trabajos. Por lo que se entiende, que en el trabajo que aquí presentamos hemos utilizado proporcionalmente más resecciones pancreáticas para tratar patología no pancreática (tumor duodenal, colangiocarcinoma distal...).

- La frecuencia cardiaca y la tensión arterial sistólica fuera de su rango establecido como normal en la escala PREPARE fueron más frecuentes en este trabajo que en el comparado por Uzunoglu et al. (136). Por otra parte, Celik et al. (139) no publicaron estos datos en su artículo de validación.

- La cifra de hemoglobina considerada como normal según la escala PREPARE (11,5-17 g/dL) se notificó con mayor asiduidad en el trabajo que aquí presentamos que en el trabajo publicado por Uzunoglu et al. (136).

- La escala del riesgo anestésico de la American Society of Anesthesiologists, (ASA), es claramente diferente comparándola como los trabajos del grupo alemán y turco. Entre ellos, esta variable es muy similar, sin embargo, en nuestro análisis solo el 33\% de los pacientes intervenidos tuvo un ASA $\geq$ III. Uzunoglu et al. (136) y Celik et al. no publicaron la comorbilidad asociada o la situación basal que tenían los pacientes a los que se les iba a realizar la resección pancreática, por ello pensamos que debido a que la distribución de 
paciente con una escala de riesgo ASA $\geq$ III es superior en los anteriores trabajos mencionados, la comorbilidad asociada en ellos es también superior.

Tabla 20: Comparación de los resultados obtenidos en la escala PREPARE por los datos propios, Uzunoglu et al. y Celik et al.

\begin{tabular}{|c|c|c|c|c|}
\hline \multicolumn{2}{|c|}{$\begin{array}{c}\text { VARIABLE } \\
\text { ESCALA PREPARE }\end{array}$} & \multirow{2}{*}{$\begin{array}{c}\text { SG } \\
75,3 \%\end{array}$} & \multirow{2}{*}{$\begin{array}{c}\begin{array}{c}\text { Serie } \\
\text { Uzunoglu }\end{array} \\
\text { al. }(136)\end{array}$} & \multirow{2}{*}{$\begin{array}{c}\begin{array}{c}\text { Serie Celik } \\
\text { et al. (139) }\end{array} \\
\text { No notificado }\end{array}$} \\
\hline Albúmina & $\geq 3,5 \mathrm{~g} / \mathrm{dL}$ & & & \\
\hline & $<3,5 \mathrm{~g} / \mathrm{dL}$ & $24,7 \%$ & $17 \%$ & No notificado \\
\hline \multirow{2}{*}{$\begin{array}{c}\text { Cirugía } \\
\text { programada }\end{array}$} & Sí & $100 \%$ & $96,4 \%$ & $93,4 \%$ \\
\hline & No (urgente) & $0 \%$ & $3,6 \%$ & $6,6 \%$ \\
\hline \multirow{2}{*}{$\begin{array}{c}\text { Técnica } \\
\text { quirúrgica }\end{array}$} & Otras técnicas & $23,7 \%$ & $27,4 \%$ & $19,7 \%$ \\
\hline & $\begin{array}{l}\text { DPC o resección } \\
\text { de cabeza de } \\
\text { páncreas con } \\
\text { preservación del } \\
\text { duodeno }\end{array}$ & $76,3 \%$ & $72,6 \%$ & $80,3 \%$ \\
\hline \multirow{2}{*}{$\begin{array}{c}\text { Patología de } \\
\text { origen } \\
\text { pancreático }\end{array}$} & Sí & $66 \%$ & $90,4 \%$ & $77,9 \%$ \\
\hline & No & $34 \%$ & $9,6 \%$ & $22,1 \%$ \\
\hline \multirow{2}{*}{$\begin{array}{c}\text { Frecuencia } \\
\text { cardíaca }\end{array}$} & 50-80 lpm & $57,7 \%$ & $77,3 \%$ & No notificado \\
\hline & $<50$ o $>80 \mathrm{lpm}$ & $42,3 \%$ & $22,7 \%$ & No notificado \\
\hline \multirow{2}{*}{$\begin{array}{l}\text { Presión } \\
\text { arterial } \\
\text { sistólica }\end{array}$} & $110-130 \mathrm{mmHg}$ & $52,6 \%$ & $59,2 \%$ & No notificado \\
\hline & $\begin{array}{c}<110 \text { o }>130 \\
\mathrm{mmHg}\end{array}$ & $47,4 \%$ & $40,8 \%$ & No notificado \\
\hline \multirow{2}{*}{$\begin{array}{c}\text { Hemoglobina } \\
\text { en sangre }\end{array}$} & $11,5-17 \mathrm{~g} / \mathrm{dL}$ & $86,6 \%$ & $52,4 \%$ & No notificado \\
\hline & $<11,5$ o $>17 \mathrm{~g} / \mathrm{dL}$ & $13,4 \%$ & $47,6 \%$ & No notificado \\
\hline \multirow{2}{*}{$\begin{array}{c}\text { Riesgo } \\
\text { anestésico } \\
\text { (escala ASA) }\end{array}$} & $\leq \mathrm{II}$ & $77 \%$ & $53,4 \%$ & $55,7 \%$ \\
\hline & $\geq \mathrm{III}$ & $33 \%$ & $46,6 \%$ & $44,3 \%$ \\
\hline
\end{tabular}

Latidos por minuto (lpm), milímetros de mercurio $(\mathrm{mmHg})$. 
En la tabla 21 se muestra la comparativa de la distribución de los pacientes en esta SG en las tres categorías de riesgo, respecto a los datos notificados por Uzunoglu et al. (136) y Celik et al. (139)

\begin{tabular}{|c|c|c|c|}
\hline \multirow{2}{*}{$\begin{array}{l}\text { CATEGORÍAS } \\
\text { DE LA ESCALA } \\
\text { PREPARE }\end{array}$} & \multicolumn{3}{|c|}{ PROPORCIÓN DE PACIENTES } \\
\hline & Datos propios & Uzunoglu et al. (136) & Celik et al. (139) \\
\hline Riesgo bajo & $56,7 \%$ & $69,3 \%$ & $3,3 \%$ \\
\hline Riesgo medio & $29,9 \%$ & $19,7 \%$ & $31,1 \%$ \\
\hline Riesgo alto & $13,4 \%$ & $11,0 \%$ & $65,5 \%$ \\
\hline
\end{tabular}

En cuanto al análisis descriptivo de las variables intraoperatorias, el 20,6\% de los pacientes tuvieron resección de bazo asociado a la resección pancreática. Sin embargo, eliminando a los pacientes a los que se había realizado DPC, el 87\% de las resecciones pancreáticas restantes tuvieron esplenectomía asociada, todas ellas DPT y pancreatectomía distal (PD). Aunque la realización de la esplenectomía está recomendada durante la resección de tumores pancreáticos de cuerpo y cola con el objetivo de realizar la linfadenectomía del grupo 10, no existe un nivel 1 de evidencia para realizar este procedimiento (148).

En este trabajo se obtuvo un $8,2 \%$ de transfusión intraoperatoria, existen publicaciones que relacionan la preservación esplénica con menor transfusión intraoperatoria y morbilidad postoperatoria (149). La técnica de Warshaw et al. (96), descrita en el apartado de introducción, puede ser un excelente recurso para preservar el bazo. 
El abordaje laparotómico fue el más frecuente. Tan solo un 5,2\% de los pacientes fue intervenido mediante abordaje laparoscópico. Análisis observacionales parecen señalar que el abordaje laparoscópico (92) o incluso el robótico (94) pueden disminuir la morbilidad postoperatoria y la estancia hospitalaria.

En cuanto a la colocación de drenajes, en ninguno de los 5 pacientes a los que se realizó DPT se dejó drenaje intraabdominal. De los 92 pacientes restantes, el 89,1\% tuvieron drenaje intraabdominal a criterio del cirujano responsable de la operación. En un artículo publicado por Kantor et al. (134) cuyo objetivo fue determinar una escala pronóstica de FPPO, en el 61,4\% de los pacientes dejó drenaje intraabdominal. En una segunda fase de ese mismo estudio, para determinar su validación externa, este porcentaje ascendió a 87,2\%. Según la última actualización del International Study Group of Pancreatic Surgery (ISGPS), se recomienda no utilizar un dispositivo de drenaje en aquellos pacientes con un grado de riesgo bajo o moderado de FPPO, únicamente reservando su uso para aquellos pacientes con alto riesgo de complicación, realizando una determinación de amilasa en el líquido de drenaje el tercer día postoperatorio (DPO), si esta es tres veces menor al límite alto de la normalidad del laboratorio de referencia, el drenaje puede retirarse (83). Un ensayo clínico alemán (PANDRA), ha demostrado mayores tasas de reintervención y FPPO en el grupo de pacientes con drenaje (88). Según un artículo español (150), la morbimortalidad postoperatoria es menor en el grupo de pacientes en los que no se utilizó drenaje intraabdominal tras DPC. En una encuesta nacional sobre las unidades de cirugía pancreática se concluyó que la política del no uso de drenajes en España está muy poco extendida ya que únicamente el 4,3\% de los 69 hospitales que participaron en ella, respondieron que no lo colocaron (151). 
A continuación, se discutirán nuestros resultados obtenidos en variables postoperatorias.

La estancia postoperatoria en la unidad de reanimación postquirúrgica obtuvo una mediana de 3 días y un rango intercuartílico (RIQ) de 2-3 días. Todos los pacientes, excepto uno que fue Clavien V, consiguieron tolerancia oral con una mediana de 4 días y un RIQ de 3-7 días. El 85,5\% de los pacientes precisaron NPT durante el postoperatorio. En los últimos años han aparecido artículos fomentando el uso de la rehabilitación multimodal. El mismo estudio español citado anteriormente (150), evalúa un programa de recuperación temprana "fast-track". Estos autores concluyen que romper con los dogmas de ayuno y estancia prolongados en la unidad de reanimación postquirúrgica y alta tardía aporta un beneficio marcado reduciendo la incidencia de morbilidad postoperatoria.

La tasa global de complicaciones globales fue $75,3 \%$, aunque esta cifra pueda parecer excesiva en cualquier procedimiento quirúrgico, grandes centros de referencia internacionales en cirugía hepatobiliopancreática han publicado cifras similares tal y como se observa en el gráfico 25 . Precisamente, los grupos más similares a los resultados aportados por nuestro trabajo son los de Wiltberger et al. (137) Braga et al. (130) y Uzunoglu et al. (136). Los dos primeros evalúan únicamente a pacientes sometidos a DPC mientras que el grupo alemán analiza una serie de enfermos sometidos a cualquier resección pancreática. 
Gráfico 25: Comparación de nuestros propios resultados con la literatura en cuanto a la tasa de complicaciones globales en pacientes intervenidos de resección pancreática mayor $(129,130,136,137,152,153)$.

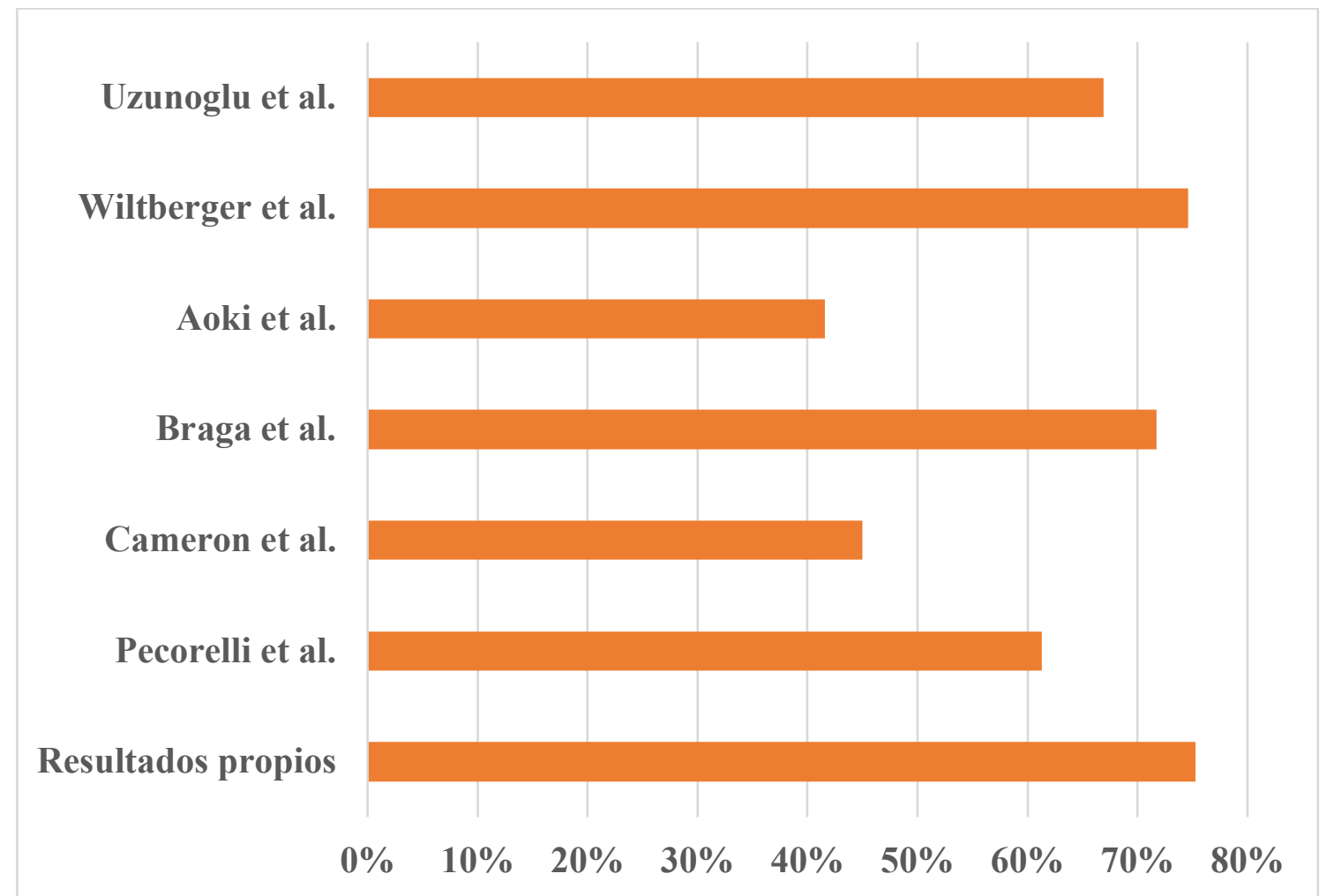

Por otra parte, en el gráfico 26 puede observarse el desglose de esas complicaciones en cada uno de los grados según la clasificación de Dindo et al. (114) comparado con el trabajo de Uzunoglu et al. (136). Desafortunadamente el trabajo de Celik et al. (139) no ha notificado estos datos desglosados en su artículo. Estos resultados en términos de morbimortalidad postoperatoria, son, al igual que ocurre para los demográficos, similares a los presentados por Celik et al. (139) y por Uzunoglu et al. (136). En esta SG de 97 pacientes, menos de 1 de cada 4 pacientes $(24,7 \%)$, evolucionó con un postoperatorio sin ningún tipo de evento adverso, cifra próxima a la expuesta en el artículo del PREPARE (27,6\%), y sin duda más favorable que la publicada por el grupo 
turco ya que todos los pacientes de esa serie presentaron alguna complicación leve o grave. En cuanto a las complicaciones graves definidas según la clasificación de DindoClavien (114) como grados IIIa, IIIb, IV y V, la proporción de individuos en la SG $(26,8 \%)$ es también similar, o incluso discretamente inferior, a la notificada en los mencionados trabajos 33\% (136) y 28,7\% (139), respectivamente. Idéntica situación existe para la mortalidad postoperatoria: $5,2 \%$ en nuestro trabajo y $5,6 \%$ en los centros europeos participantes en el artículo del PREPARE y, por desgracia, el grupo turco no notificó este dato en su estudio. En un estudio alemán publicado en el año 2016 con una extensísima serie de resecciones pancreáticas (58003, durante 4 años), obtuvieron un $10,1 \%$ de mortalidad intrahospitalaria (124), cifra dos veces superior a la aportada en este análisis.

Llama especialmente la atención en el gráfico 26, La similitud de la proporción en todos los grados excepto la diferencia que puede observarse en el grado de Clavien IIIb. En la definición de este grado se incluyen a los enfermos en los que ha sido necesaria una reintervención quirúrgica. La causa de esta reintervención fue por FPPO y HPP, ambas condiciones pueden ser precursoras de fallo orgánico precisando de esta manera el ingreso asociado en una unidad de cuidados intensivos en numerosas ocasiones, lo que hace ascender el grado de complicación a Clavien IV que precisamente en nuestro estudio es ligeramente superior al aportado por el grupo alemán. 
Gráfico 26.: Comparación de nuestros propios resultados con la literatura en cuanto al grado de complicaciones según la escala de Dindo et al. (114) en pacientes intervenidos de resección pancreática mayor.

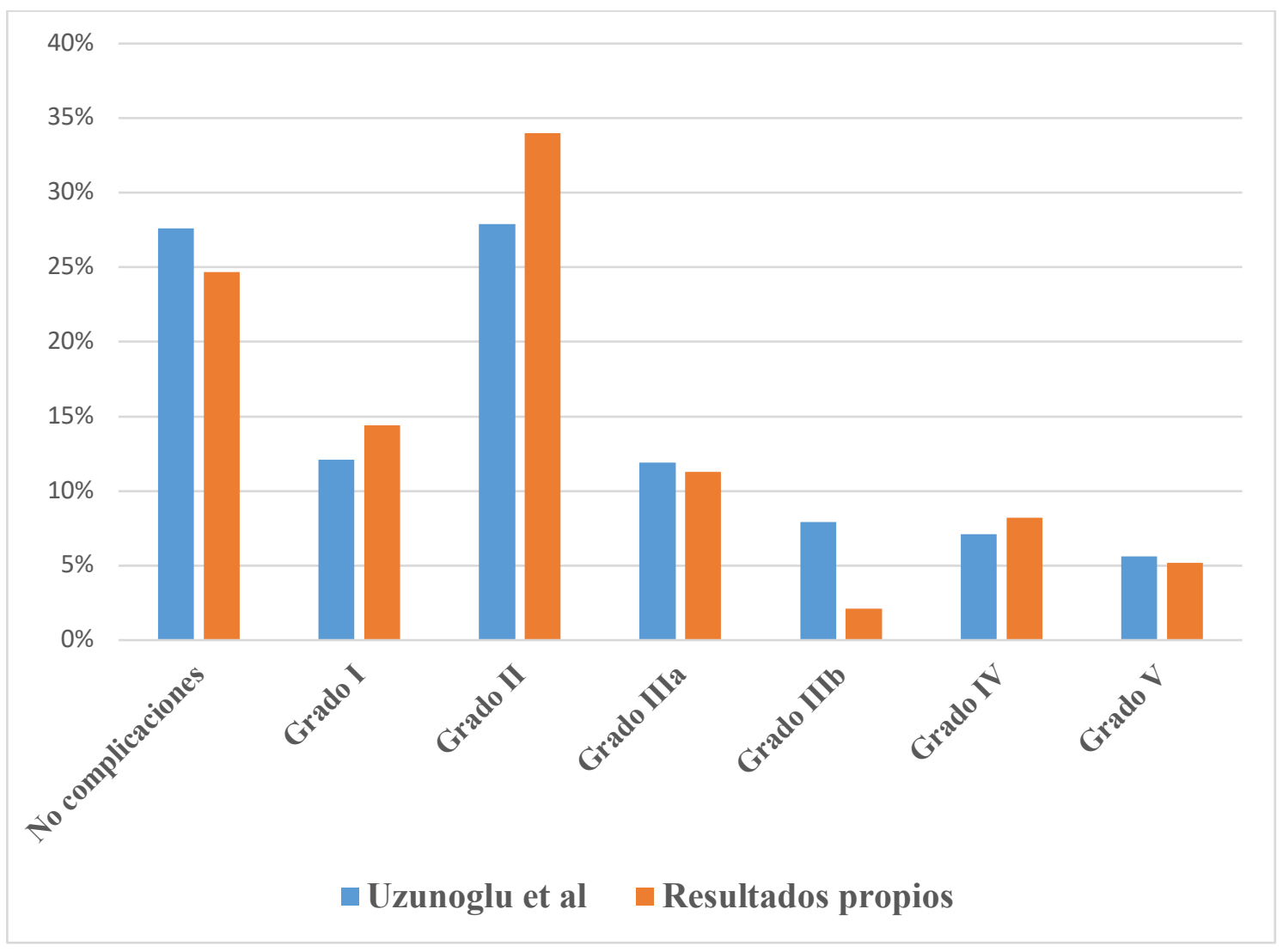

Puede resultar interesante comparar estos resultados con los notificados en otro estudio alemán muy recientemente publicado por el grupo de Krautz et al. (154), en el que se exponen las diferencias en morbilidad postoperatoria y mortalidad entre hospitales con alto volumen de resecciones pancreáticas mayores y hospitales con bajo volumen de las mismas (las divide en 5 grupos, desde muy poco volumen hasta muy alto volumen). La mortalidad postoperatoria ajustada por riesgo en hospitales con muy alto volumen frente a hospitales con muy bajo volumen fue de 6,5\% frente a $11,5 \%$. En ese mismo estudio, la mediana de la estancia postoperatoria difiere en 5 días de hospitales con muy poco volumen 25 y un RIQ de 17-36 a 20 y un RIQ de 14-30 en hospitales con muy alto 
volumen (154). Según otro estudio (155) en el que está basada la clasificación del grado de volumen de hospitales analizados por el grupo de Krautz et al. (154), nuestra unidad en el Hospital Universitario Rio Hortega (HURH) estaría catalogada en el grupo de hospitales con muy alto volumen (17,6 casos por año). Si observamos el trabajo de Krautz et al. (154), la mortalidad que debería tener el HURH estaría alrededor de 6,1\%. En el presente trabajo, como se ha mencionado previamente, la mortalidad es de 5,2\%.

Por otra parte, el concepto de alto volumen no está consensuado. Un hospital de muy alto volumen, es aquel donde se realizan más de 16 resecciones pancreáticas mayores al año (155). Sin embargo, según Cameron et al. (152) un cirujano debe realizar al menos 15 intervenciones al año para ser considerado de alto volumen.

A continuación, los gráficos 27, 28 y 29 muestra los resultados de las complicaciones específicas obtenidas en este trabajo (FPPO, RVG Y HPP) comparado con lo publicado en la literatura científica. Además, en el gráfico 27 que muestra la tasa FPPO, también se compara la tasa de fístula pancreática clínicamente relevante (FPCR).

La tasa de FPPO es ligeramente inferior a la publicada por Braga et al. (130) y Sánchez-Cabús et al. (156). Sin embargo, es superior a la tasa publicada por el resto de grupos de referencia en cirugía pancreática. Por otra parte, la tasa FPCR en este estudio es inferior a la mayoría de estos centros de referencia americanos, europeos y españoles, únicamente superada por la aportada por Dominguez-Comesaña et al. (157). Recientemente Pedrazzoli et al.(158) publicaron la revisión más extensa aportada hasta el momento a sobre FPPO. La tasa entre los diferentes estudios es muy variable, oscilando entre el 3\% y más del 40\%. 
Gráfico 27: Comparación de nuestros propios resultados con la literatura en cuanto a la tasa de FPPO y FPCR $(130,131,136,156,157,159-162)$

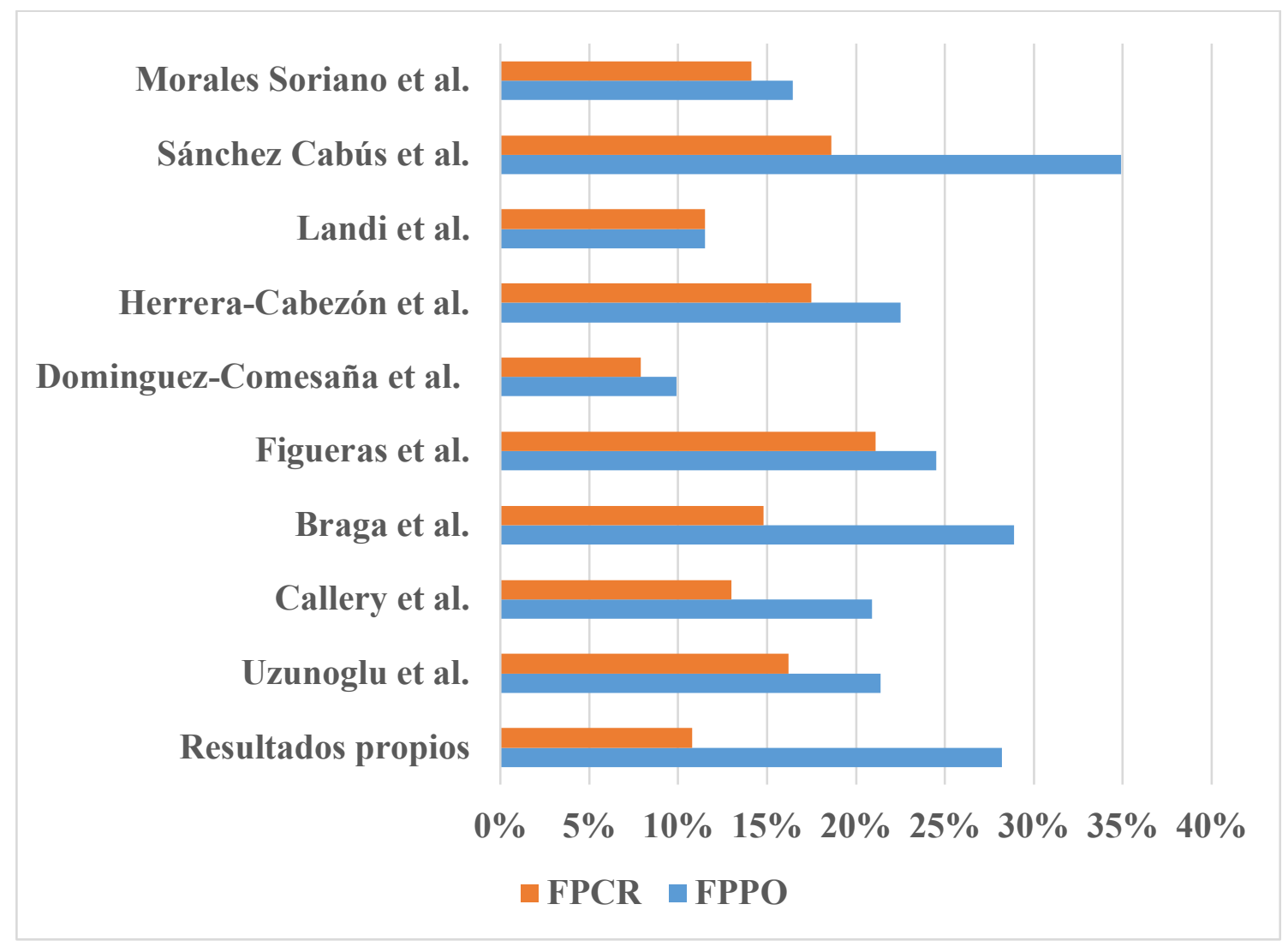

La tasa de RVG notificada en este trabajo fue del 26,8\%. Sin embargo, según Wente et al. (121) la incidencia del RVG difiere mucho entre grupos ya que aunque exista un consenso internacional, no todos los autores lo aplican, por lo que estas tasas se sitúan entre el $19 \%$ y $57 \%$. Por otra parte, se puede observar que este rango tampoco se cumple ya que existen autores que publican datos inferiores al límite establecido por la ISGPS como se muestra en el gráfico 28. 
Gráfico 28: Comparación de nuestros propios resultados con la literatura en cuanto a la tasa de RVG $(78,130,152,156,160-164)$.

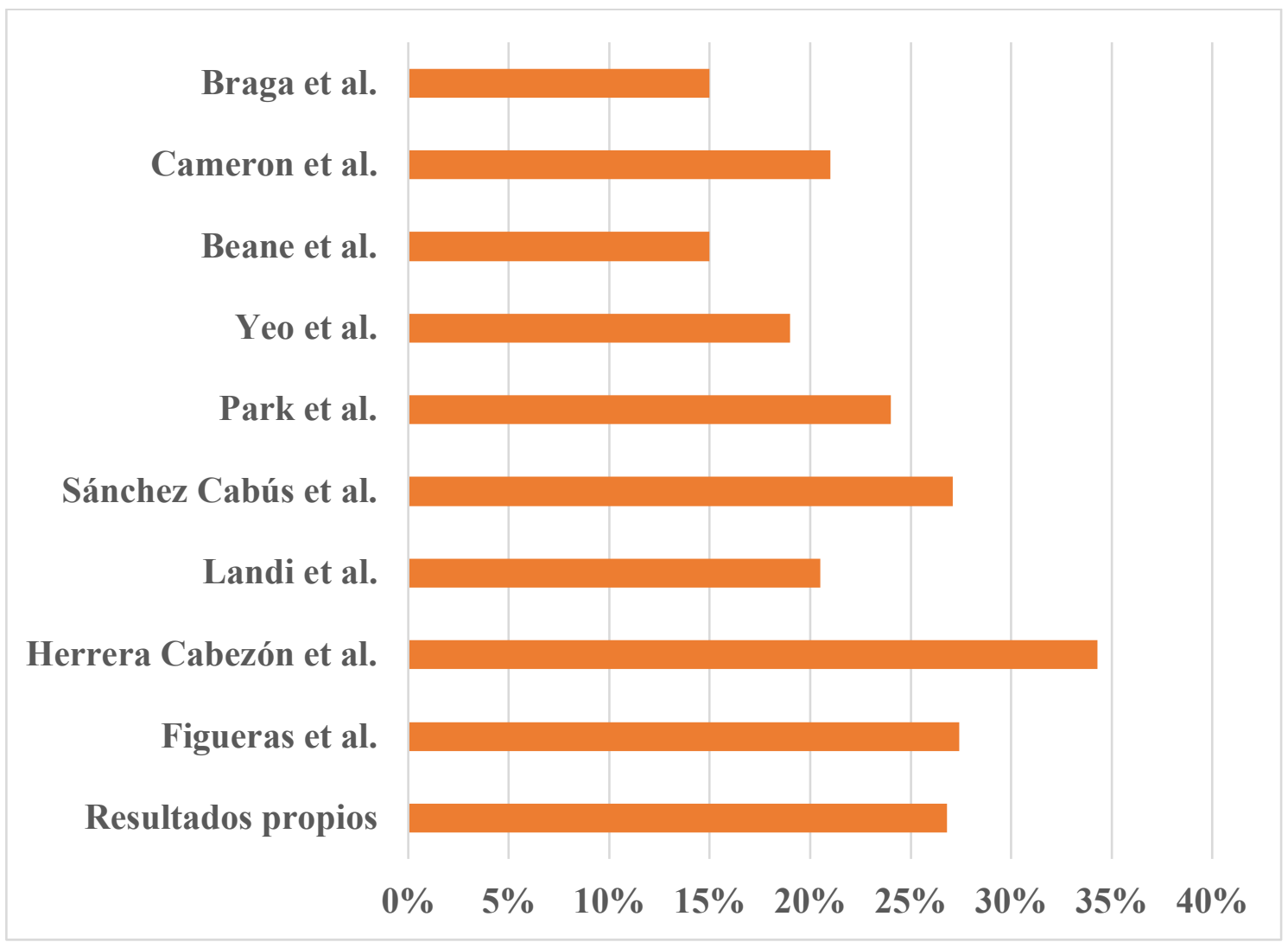

La tasa de HPP notificada en este trabajo fue de 18,2\%, porcentaje superior a los trabajos revisados. Desglosando este porcentaje, el 13,4\% corresponde a los grados B y C de la clasificación ISGPS (128). Aunque este consenso internacional se utiliza con asiduidad, a día de hoy muchas publicaciones continúan sin homogeneizar su metodología dificultando de esta manera la comparabilidad de los resultados obtenidos. Duarte et al. (165) recientemente han publicado una revisión sugiriendo replantear nuevamente la definición de HPP, ya que aquellos pacientes con HPP grado A y los que no tuvieron hemorragia obtenían las mismas tasas de morbimortalidad postoperatoria. 
Gráfico 29: Comparación de nuestros propios resultados con la literatura en cuanto a la tasa de HPP $(130,136,156,157,160,162,165,166)$.

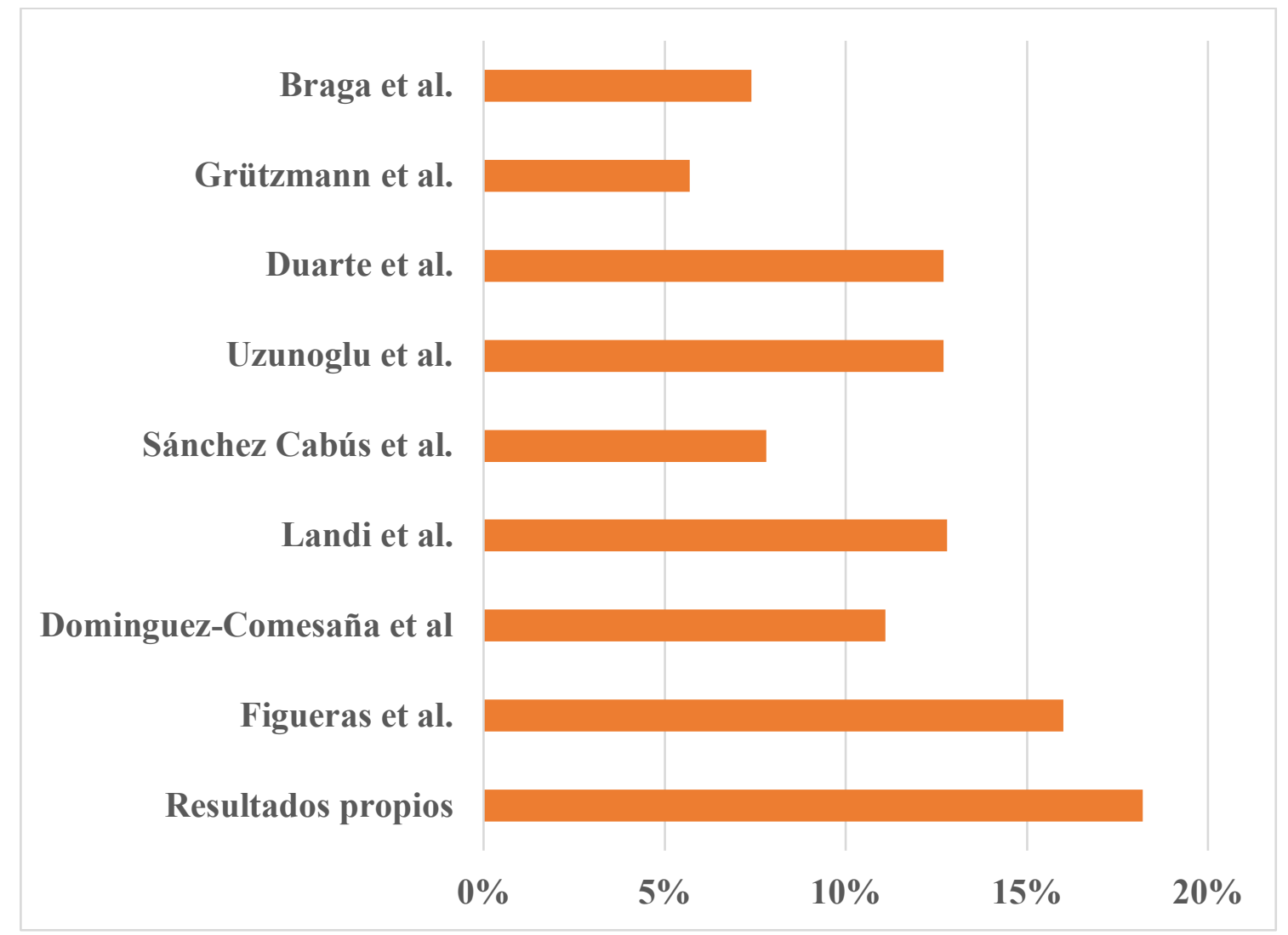

La tasa de IHQ en la SG de este trabajo fue del 17,5\% de los pacientes, tasa muy similar a la revisión publicada por Angiolini et al. (167) cuya cifra de IHQ superficial y profunda fue del 17,6\%. Greenblaltt et al. (133) notificaron un 13,5\% de IHQ únicamente superficial. Finalmente, el grupo japonés de Aoki et al.(129) publicaron su tasa de IHQ profunda y superficial en el $11,5 \%$ de los enfermos.

La tasa de reintervención del 8,2\% fue superior a la de otras grandes series. $(129,133,154,168)$. Por otra parte esta cifra es inferior a otras series de grupos españoles $(156,157,159-161,169)$. 
La mediana de estancia postoperatoria global fue de 11 días, esta cifra es claramente inferior a la mayoría de series publicadas hasta el momento [Tabla 22].

\begin{tabular}{|l|l|}
\hline \multicolumn{2}{|l|}{$\begin{array}{l}\text { Tabla 22: comparación de nuestros propios resultados con la literatura en cuanto } \\
\text { a la tasa de estancia postoperatoria global }(78,131,156,159-162,169) .\end{array}$} \\
\hline Callery et al. & Media de 8 días. \\
\hline Yeo et al. & Mediana de 13 días. \\
\hline Figueras et al. & Mediana de 14 días. \\
\hline Herrera-cabezón et al. & Mediana de 17 días. \\
\hline Rubio-Manzanares Dorado et al. & Mediana de 18 días. \\
\hline Landi et al. & Mediana de 19 días. \\
\hline Sánchez Cabús et al. & Mediana de 24 días. \\
\hline Morales Soriano et al. & Mediana de 16 días. \\
\hline Resultados propios. & Mediana de 11 días. \\
\hline
\end{tabular}

Recientemente Sabater et al. (170) publicaron en la revista Cirugía Española, un artículo cuyo objetivo fue identificar los indicadores de calidad en la cirugía pancreática oncológica en España, así como sus límites de variabilidad. En este artículo concluyeron que los indicadores más relevantes son los que se analizan en la tabla 23.

En nuestro estudio basándose en los estándares de calidad propuestos por Sabater et al. (170), cumple perfectamente con todos los indicadores de calidad excepto en la morbilidad global. Se debe resaltar que este análisis es prospectivo, con una recogida de datos muy estricta, ya que no se ha perdido ninguna variable en ningún paciente. Además,se ha prestado mucha atención en el más mínimo detalle sucedido durante postoperatorio, el simple ejemplo de prescribir fisioterapia respiratoria o la reposición 
hidroelectrolítica para tratar una arritmia se considera como un grado I de la clasificación de Dindo et al. (114).

\begin{tabular}{|c|c|c|c|}
\hline & $\begin{array}{l}\text { Media de todos los } \\
\text { trabajos revisados }\end{array}$ & $\begin{array}{c}\text { Resultados } \\
\text { propios }\end{array}$ & Límite de calidad \\
\hline Morbilidad global & $58 \%$ & $75,3 \%$ & $<73 \%$ \\
\hline Mortalidad & $4 \%$ & $5,2 \%$ & $<10 \%$ \\
\hline Tasa de FPPO & $18 \%$ & $28,2 \%$ & $<29 \%$ \\
\hline Tasa de HPP & $11 \%$ & $18,2 \%$ & $<21 \%$ \\
\hline Reintervención & $11 \%$ & $8,2 \%$ & $<20 \%$ \\
\hline $\begin{array}{c}\text { Estancia } \\
\text { postoperatoria } \\
\text { global }\end{array}$ & Mediana de 18 días & Mediana de 11 días & $<21$ días \\
\hline
\end{tabular}

\subsection{2.- ANÁLISIS DE LA VALIDACIÓN DE LA ESCALA PREPARE}

La escala PREPARE fue definida, como ya se ha descrito anteriormente, con el objetivo de pronosticar de manera preoperatoria el riesgo que tiene un paciente que va a ser sometido a resección pancreática mayor de tener una complicación grave (Clavien $\geq$ IIIa). Este trabajo que publicado por Uzunoglu et al. (136) utiliza una serie de variables preoperatorias cuya puntuación final estratifica al paciente en un grado de riesgo prequirúrgico para sufrir una complicación grave. [Tabla 12 y figura 10 en el apartado de Introducción].

El trabajo original de la escala PREPARE de Uzunoglu et al. (136) fue realizado en 703 pacientes de manera retrospectiva con la finalidad de identificar variables de 
riesgo para formular la escala PREPARE. Finalmente, una vez identificadas esas variables, se realizó en un segundo tiempo una reclutación de 429 pacientes para realizar una validación interna prospectiva de esa nueva escala pronóstica de riesgo. Se obtuvo de tal manera un tamaño muestral de 1132 pacientes. En cuanto a las características principales de esta escala, destacan las siguientes:

- Incluye 8 variables clínicas de fácil determinación: cifra de albúmina plasmática, realización de cirugía urgente o electiva, frecuencia cardiaca y presión arterial sistólica del paciente, técnica quirúrgica, patología estrictamente pancreática o de otra etiología, cifra de hemoglobina en sangre y grado en la escala de riesgo anestésico ASA. En función de los datos del paciente, se asigna o no una puntuación concreta por cada una de las variables [tabla 12 de la Introducción]. De todas ellas, la que más puntos aporta es una cifra de albúmina inferior a 3,5 g/dL, siendo ésta un indicador de mala situación nutricional del paciente. Por otra parte, la inclusión de la variable "técnica quirúrgica" resulta de gran interés, puesto que los distintos procedimientos descritos en cirugía pancreática se asocian, también, con diferente tasa de complicaciones. En concreto la DPC, en cualquiera de sus variantes técnicas, supone 2 puntos por el riesgo inherente de la anastomosis pancreática.

- El registro de complicaciones se hizo empleando la clasificación de DindoClavien (114), lo cual supone homogeneidad y exhaustividad en la definición de la severidad de las mismas, así como una fácil reproductibilidad por parte de otros investigadores. Se definió la complicación mayor con una puntuación de Clavien $\geq$ IIIa. 
- El cálculo de la puntuación de la escala PREPARE se realiza antes de la intervención quirúrgica, empleando las mencionadas variables que son determinadas preoperatoriamente. De acuerdo a los puntos obtenidos se establecen 3 grupos de riesgo: bajo, medio y alto [figura 10 de la Introducción].

El primer grupo en realizar una validación externa de esta escala fue el grupo turco de Celik et al. (139) a principios del año 2016 en la revista Hepatobiliary and Pancreatic Diseases International. En este trabajo seleccionaron a 122 pacientes sometidos a cirugía pancreática entre los años 2010 y 2015 realizando un análisis retrospectivo de los mismos.

Unos meses más tarde, a finales del año 2016, en esa misma revista, nuestro grupo realizó una nueva validación externa de la escala PREPARE (140). En esta ocasión se incluyeron únicamente 18 pacientes ya que se trataba de una validación preliminar. La ventaja de este estudio es que todos los pacientes fueron analizados de manera prospectiva. Finalmente, dos años más tarde, nuestro grupo publicó una nueva validación externa con la inclusión final de 50 pacientes analizados también de manera prospectiva (138).

En el año 2018 Samim et al. (141) publicaron un estudio cuyo objetivo fue evaluar si las escalas de predicción de riesgo prequirúrgico en cirugía hepatobiliopancreática superaban a la evaluación de riesgo realizada subjetivamente por el cirujano. Entre las escalas de riesgo que se evaluaron, incluyeron a 193 pacientes para evaluar la escala PREPARE en la parte de evaluación de riesgo cirugía pancreática. 
En la aparición de complicaciones tras cirugía de páncreas, no sólo influye la propia dificultad técnica inherente a estos procedimientos, sino también las características y situación clínica basales de cada paciente. De ahí que la escala PREPARE resulte útil para comparar la tasa de complicaciones estratificando a los pacientes según las 3 categorías de riesgo.

Esto resulta más preciso que emplear las cifras brutas y globales de toda una muestra con pacientes heterogéneos. En este sentido, la tabla 24 muestra que, aunque la proporción de pacientes incluidos en las categorías de riesgo del PREPARE difiere llamativamente en las 3 series comparadas [datos de este estudio en la SG, datos de Uzunoglu et al. (136) y datos de Celik et al. (139)], el porcentaje de complicaciones graves es similar, lo que avala una correcta capacidad predictiva de esta escala. Samim et al. (141) no notificaron estos datos en su estudio.

Tabla 24: comparativa de la distribución del porcentaje de pacientes y su correspondiente proporción de complicaciones graves según las categorías de la escala PREPARE en la SG frente a las series de Uzunoglu et al. (136) y Celik et al. (139).

\begin{tabular}{|c|c|c|c|c|c|c|}
\hline & \multicolumn{3}{|c|}{$\begin{array}{c}\text { PROPORCIÓN DE } \\
\text { PACIENTES }\end{array}$} & \multicolumn{3}{c|}{$\begin{array}{c}\text { COMPLICACIONES } \\
\text { GRAVES }\end{array}$} \\
\hline $\begin{array}{c}\text { CATEGORÍAS } \\
\text { DE LA } \\
\text { ESCALA } \\
\text { PREPARE }\end{array}$ & $\begin{array}{c}\text { Datos } \\
\text { propios }\end{array}$ & $\begin{array}{c}\text { Uzunoglu } \\
\text { et al. }\end{array}$ & $\begin{array}{c}\text { Celik } \\
\text { et al. }\end{array}$ & $\begin{array}{c}\text { Datos } \\
\text { propios }\end{array}$ & $\begin{array}{c}\text { Uzunoglu } \\
\text { et al. }\end{array}$ & $\begin{array}{c}\text { Celik } \\
\text { et al. }\end{array}$ \\
\cline { 2 - 7 } Riesgo bajo & $56,7 \%$ & $69,3 \%$ & $3,3 \%$ & $18,2 \%$ & $18 \%$ & $0 \%$ \\
\hline Riesgo medio & $29,9 \%$ & $19,7 \%$ & $31,1 \%$ & $24,1 \%$ & $40,0 \%$ & $31,4 \%$ \\
\hline Riesgo alto & $13,4 \%$ & $11,0 \%$ & $65,5 \%$ & $69,2 \%$ & $66,0 \%$ & $68,6 \%$ \\
& & & & & & \\
\hline
\end{tabular}


La escala de PREPARE en la SG logra diferenciar y estratificar adecuadamente a los pacientes en este estudio (las categorías de bajo y alto riesgo acumula el 70,1\%), cifra similar a lo publicado por los autores que la describieron con una proporción del 75,5\% (136) (en el grupo de validación interna de los 429 pacientes), al igual que notificó el grupo turco $(68,9 \%)(139)$. Estos datos, resulta de gran valor e interés estadístico, ya que supone una distribución adecuada del tamaño muestral entre las categorías de riesgo.

Para validar esta escala de riesgo, en términos de discriminación (grado en que el modelo distingue entre individuos en los que ocurre el evento y los que no), se ha realizado una curva Receiver Operating Characteristic (ROC). La discriminación aportada por la escala de PREPARE en la SG es muy favorable a la vista del AUC obtenido. Para completar la validación estadística se realizó un test de calibración (grado en que la probabilidad estimada por la prueba coincide con la realmente observada) empleando el test de bondad de ajuste de Hosmer-Lemeshow. Estos resultados fueron favorables ya que la no significación estadística implica que no existan diferencias entre el resultado esperado y obtenido. La tabla 25 muestra la comparación de datos relativos a la validación de la escala $P R E P A R E$ en los resultados propios de la SG, Uzunoglu et al. (136), Celik et al (139) y Samim et al. (141) 


\begin{tabular}{|c|c|c|c|c|}
\hline \multicolumn{5}{|c|}{$\begin{array}{l}\text { Tabla 25: Tabla comparativa de los datos estadísticos relativos a la validación de } \\
\text { la escala PREPARE en la SG frente a las series de Uzunoglu et al. (136), Celik et } \\
\text { al. (139) y Samim et al. (141) }\end{array}$} \\
\hline $\begin{array}{c}\text { DATOS } \\
\text { ESTADÍSTICOS }\end{array}$ & $\begin{array}{l}\text { AUC en la } \\
\text { curva ROC }\end{array}$ & $\begin{array}{l}\text { Proporción en } \\
\text { las categorías } \\
\text { de bajo y alto } \\
\text { riesgo }\end{array}$ & $\begin{array}{c}\text { Test de } \\
\text { Hosmer- } \\
\text { Lemeshow }\end{array}$ & $\begin{array}{l}\text { Porcentaje } \\
\text { pronosticado } \\
\text { correcto }\end{array}$ \\
\hline $\begin{array}{l}\text { RESULTADOS } \\
\text { PROPIOS } \\
\text { PREPARE } \\
\text { SERIE GLOBAL }\end{array}$ & $\begin{array}{c}0,671 \\
(\mathrm{IC} 95 \%= \\
0,539-0,802 \\
\mathrm{p}=0,01)\end{array}$ & $70,1 \%$ & $\mathrm{p}=0,147$ & $78,4 \%$ \\
\hline UZUNOGLU et al. * & $\begin{array}{c}0,709 \\
(\mathrm{IC} 95 \%= \\
0,657-0,760 \\
\mathrm{p}=0,001)\end{array}$ & $75,5 \%$ & $\mathrm{p}=0,454$ & $75 \%$ \\
\hline CELIK et al. & $\begin{array}{l}0,541 \\
(\mathrm{IC} 95 \% \text { no } \\
\text { notificado) }\end{array}$ & $68,9 \%$ & $p=0,764$ & $70 \%$ \\
\hline SAMIM et al. & $\begin{array}{c}0,51 \\
(\mathrm{IC} 95 \%= \\
0,39-0,6)\end{array}$ & $\begin{array}{c}\text { No } \\
\text { notificado }\end{array}$ & $\begin{array}{c}\text { No } \\
\text { notificado }\end{array}$ & No notificado \\
\hline
\end{tabular}

AUC (Area Under Curve), IC 95\% (intervalo de confianza al $95 \%)$,

* Datos correspondientes a la cohorte prospectiva de validación de la escala, que incluye 429 pacientes.

\subsection{3.- CORELACIÓN DE LA ESCALA PREPARE CON OTRAS}

\section{VARIABLES DE RESULTADO}

La escala PREPARE no fue descrita para predecir otros datos como complicaciones globales, complicaciones específicas postpancreatectomía, reintervención, mortalidad postoperatoria ni IHQ. Sin embargo, en este trabajo se ha querido relacionar si los grados de estadificación de riesgo prequirúrgicos se asocian a la aparición de alguna de estas variables.

El riesgo alto prequirúrgico se asoció a la aparición de complicaciones globales. Es decir, todos los pacientes que tenían un riesgo alto prequirúrgico de sufrir 
complicaciones graves desarrollaron algún tipo de complicación durante el postoperatorio. Por otra parte, Ninguno de los pacientes que se habían catalogado como bajo riesgo de sufrir complicaciones graves en la escala PREPARE, murió ni tuvo que ser reintervenido. Los grados de gravedad de FPPO se asociaron también a los grados de gravedad de la escala PREPARE.

En la literatura médica existen otras escalas de estadificación de riesgo prequirúrgico en cuanto a la morbilidad global y la mortalidad postoperatoria.

- Parikh et al. (144) publicaron una escala de riesgo que demostró asociación con morbilidad global postoperatoria, mortalidad y complicación grave tras cualquier tipo de resección pancreática mayor.

- Are et al. (171) publicaron un estudio que evaluaba un nomograma para predecir el riesgo de mortalidad postoperatoria en cualquier resección pancreática mayor.

- Velez-Serrano et al. (172) desarrollaron un modelo capaz de pronosticar el riesgo de mortalidad postoperatoria en todas las resecciones pancreáticas mayores.

- Venkat et al. (135) publicaron una escala que analizaba una serie de variables preoperatorias e intraoperatorias. Demostraron que los datos que analizaban se asociaban a mortalidad postoperatoria a 30 y 90 días.

- Greenblatt et al. (133) consiguieron asociar una escala formada por variables preoperatorias con mortalidad postoperatoria.

\section{2.- SUBGRUPO DUODENOPANCREATECTOMÍA CEFÁLICA}

En este estudio se ha considerado introducir otra escala pronóstica de complicaciones muy novedosa, la escala Wiltberger (137). Hemos comprobado que no 
se ha publicado ninguna validación externa hasta la fecha, tras una exhaustiva búsqueda bibliográfica en la literatura científica. Por tanto, tampoco existe hasta la fecha ningún artículo publicado que evalúe la escala PREPARE y Wiltberger conjuntamente y su comparación en términos de rentabilidad pronóstica y de discriminación de los test.

En el mismo periodo de tiempo, se ha seleccionado un subgrupo de pacientes de la SG. Todos ellos fueron aquellos enfermos a los que se realizó una DPC (74 pacientes). Debido a que se estableció un análisis de un subgrupo de pacientes, los criterios de exclusión fueron los mismos que los de la SG.

En cuanto al tamaño muestral, el artículo original de Wiltberger et al. (137) describió una muestra de 405 pacientes a los que se les realizó exclusivamente DPC durante un periodo de tiempo de 21 años en un solo centro hospitalario. Fueron intervenidos 1,6 pacientes al mes, cifra muy similar a la de este trabajo que reclutó a 74 pacientes en 53 meses, 1,4 pacientes al mes. Se trató de un análisis retrospectivo. Dado que el presente trabajo es el primer estudio de validación externa de esta escala, el tamaño muestral de 74 pacientes, aunque es escaso, podría ser suficiente teniendo en cuenta el porcentaje de complicaciones Clavien $\geq$ IIIb y la ratio similar de pacientes intervenidos en nuestro estudio.

\subsection{1.- ANÁLISIS DE LOS RESULTADOS DESCRIPTIVOS}

El $56,8 \%$ de los pacientes fueron varones, porcentaje superior al notificado en la SG, pero dentro las cifras habituales publicadas $(129,130,132-137,139)$ [Gráfico 30]. Wiltberger et al. (137) comunicaron un porcentaje aún mayor, $61 \%$ de varones. 
Gráfico 30.: Comparación de nuestros propios resultados con la literatura en cuanto a la distribución por sexo en pacientes intervenidos de DPC $(129,130,132-135,137)$.

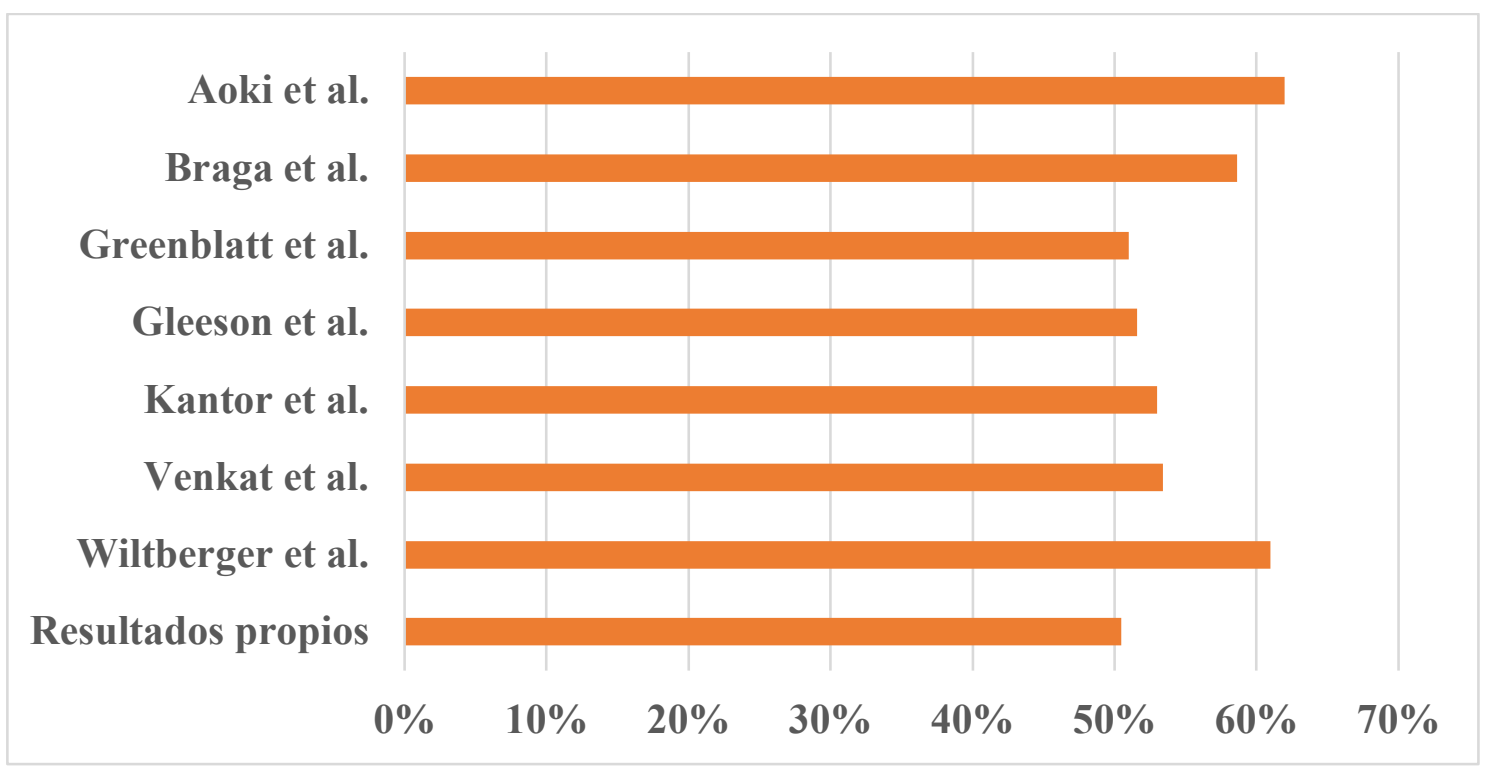

En cuanto al análisis de la edad en el SDPC, la media es ligeramente superior en los pacientes tratados con DPC que en la SG de nuestro estudio (63 frente a 61 años respectivamente). Sin embargo, esta dentro del rango de edad publicado en estudios similares $(129,130,132-135,137,139)$. Un dato a tener en cuenta es que a pesar de que la media sea distinta en la SG y el SDPC, el rango es el mismo, por lo que de esto se traduce que al paciente más joven y el más mayor se les realizó una DPC.

La prótesis biliar preoperatoria fue colocada en el $81,1 \%$ de los pacientes. Comparando esta cifra con los datos de la SG $(63,9 \%)$, se puede observar una diferencia claramente superior. Esto es lógico, ya que en las resecciones de la SG está incluida la PD, y en este tipo de cirugía es muy poco común utilizar prótesis biliar preoperatoria ya que la patología no suele afectar al conducto biliar principal. 
En la tabla 26 se puede observar una comparativa de la proporción de enfermos según la distribución por categorías de todas las variables de la escala PREPARE en los datos propios de este trabajo en la SG y el SDPC junto con lo publicado por Uzunoglu et al. (136) y Celik et al. (139). En dicha tabla, las diferencias más notables que se pueden apreciar son:

- Los pacientes del SDPC están peor nutridos en comparación con los de la SG y los del artículo original de la escala PREPARE. (136)

- La DPC es una técnica quirúrgica utilizada en múltiples ocasiones para resecar tumores duodenales adyacentes a la papila, colangiocarcinoma distal o ampuloma. Es por ello, que el porcentaje de patología de origen pancreático en este SDPC es menor que en la SG.

- Los pacientes del SDPC fueron catalogados con una puntuación ASA mayor que aquellos pertenecientes a la $\mathrm{SG}$, por lo que se deduce que tienen mayor comorbilidad prequirúrgica. 
Tabla 26: Tabla comparativa de los resultados obtenidos en la escala PREPARE por los datos propios, Uzunoglu et al. (136) y Celik et al. (139)

\begin{tabular}{|c|c|c|c|c|}
\hline \multicolumn{2}{|c|}{$\begin{array}{c}\text { VARIABLE } \\
\text { ESCALA PREPARE }\end{array}$} & \multirow{2}{*}{$\begin{array}{l}\text { SDPC } \\
68,9 \%\end{array}$} & \multirow{2}{*}{$\begin{array}{c}\text { Uzunoglu et al. } \\
83 \%\end{array}$} & \multirow{2}{*}{$\begin{array}{c}\text { Celik et al. } \\
\text { No } \\
\text { notificado }\end{array}$} \\
\hline \multirow[t]{2}{*}{$\begin{array}{l}\text { Albúmina } \\
\text { plasmática }\end{array}$} & $\geq 3,5 \mathrm{~g} / \mathrm{dL}$ & & & \\
\hline & $<3,5 \mathrm{~g} / \mathrm{dL}$ & $31,1 \%$ & $17 \%$ & $\begin{array}{c}\text { No } \\
\text { notificado }\end{array}$ \\
\hline \multirow{2}{*}{$\begin{array}{c}\text { Cirugía } \\
\text { programada }\end{array}$} & Sí & $100 \%$ & $96,4 \%$ & $93,4 \%$ \\
\hline & No (urgente) & $0 \%$ & $3,6 \%$ & $6,6 \%$ \\
\hline \multirow{2}{*}{$\begin{array}{l}\text { Técnica } \\
\text { quirúrgica }\end{array}$} & Otras técnicas & $0 \%$ & $27,4 \%$ & $19,7 \%$ \\
\hline & $\begin{array}{l}\text { DPC o resección } \\
\text { de cabeza de } \\
\text { páncreas con } \\
\text { preservación del } \\
\text { duodeno }\end{array}$ & $100 \%$ & $72,6 \%$ & $80,3 \%$ \\
\hline \multirow{2}{*}{$\begin{array}{c}\text { Patología de } \\
\text { origen } \\
\text { pancreático }\end{array}$} & Sí & $58,1 \%$ & $90,4 \%$ & $77,9 \%$ \\
\hline & No & $41,9 \%$ & $9,6 \%$ & $22,1 \%$ \\
\hline \multirow[t]{2}{*}{$\begin{array}{l}\text { Frecuencia } \\
\text { cardíaca }\end{array}$} & 50-80 lpm & $66,2 \%$ & $77,3 \%$ & $\begin{array}{c}\text { No } \\
\text { notificado }\end{array}$ \\
\hline & $<50$ o $>801 \mathrm{lpm}$ & $33,8 \%$ & $22,7 \%$ & $\begin{array}{c}\text { No } \\
\text { notificado }\end{array}$ \\
\hline \multirow[t]{2}{*}{$\begin{array}{l}\text { Presión arterial } \\
\text { sistólica }\end{array}$} & $110-130 \mathrm{mmHg}$ & $51,4 \%$ & $59,2 \%$ & $\begin{array}{c}\text { No } \\
\text { notificado }\end{array}$ \\
\hline & $\begin{array}{c}<110 \text { o }>130 \\
\mathrm{mmHg}\end{array}$ & $48,6 \%$ & $40,8 \%$ & $\begin{array}{c}\text { No } \\
\text { notificado }\end{array}$ \\
\hline \multirow[t]{2}{*}{$\begin{array}{l}\text { Hemoglobina } \\
\text { en sangre }\end{array}$} & $11,5-17 \mathrm{~g} / \mathrm{dL}$ & $85,1 \%$ & $52,4 \%$ & $\begin{array}{c}\text { No } \\
\text { notificado }\end{array}$ \\
\hline & $<11,5 \mathrm{o}>17 \mathrm{~g} / \mathrm{dL}$ & $14,9 \%$ & $47,6 \%$ & $\begin{array}{c}\text { No } \\
\text { notificado }\end{array}$ \\
\hline \multirow{2}{*}{$\begin{array}{c}\text { Riesgo } \\
\text { anestésico } \\
\text { (escala ASA) }\end{array}$} & $\leq \mathrm{II}$ & $62,2 \%$ & $53,4 \%$ & $55,7 \%$ \\
\hline & $\geq \mathrm{III}$ & $37,8 \%$ & $46,6 \%$ & $44,3 \%$ \\
\hline
\end{tabular}

En la tabla 27 se muestra una comparativa de la proporción de enfermos según la distribución por categorías de todas las variables de la escala Wiltberger en los datos 
propios de este trabajo en el SDPC junto con lo publicado en el que describió la escala (137). Los puntos que cabe destacar son:

- El Índice de masa corporal (IMC) de los pacientes de este estudio en el SDPC estuvo dividido en el 40,5\% tenían sobrepeso grado I y II y el $20,3 \%$ de los pacientes tenían obesidad. El artículo de Wiltberger et al. (137) no estratifica a los pacientes en los tres rangos en los que está dividida esta variable en la escala pronóstica. Sin embargo, Wiltberger et al. (137) publicaron un IMC medio de 24,9 kilogramos por metro cuadrado $\left(\mathrm{Kg} / \mathrm{m}^{2}\right)$ (rango de 15,9 a 45,3 $\mathrm{Kg} / \mathrm{m}^{2}$ ), mientras que los pacientes de nuestro estudio tuvieron un IMC medio de $26,3 \mathrm{Kg} / \mathrm{m}^{2}$ (rango de 17,6 a $38,8 \mathrm{Kg} / \mathrm{m}^{2}$ ).

- Los pacientes de la serie de Wiltberger et al. (137) tuvieron mayor riesgo preanestésico que nuestros pacientes del SDPC.

- Los pacientes de la SDPC tuvieron menos enfermedades cardiovasculares, pero más enfermedades respiratorias, que forman parte de la definición de ambas variables. 


\begin{tabular}{|c|c|c|c|}
\hline \multicolumn{3}{|c|}{ Tabla 27: Tabla comparativa de los resultados obtenidos en la escala } \\
Wiltberger por los datos propios en el SDPC y Wiltberger et al. \\
\hline \multicolumn{2}{|c|}{ VARIABLE ESCALA WILTBERGER } & SDPC & $\begin{array}{c}\text { Wiltberger } \text { et } \\
\text { al. }\end{array}$ \\
\hline \multirow{2}{*}{ IMC } & $<25 \mathrm{Kg} / \mathrm{m}^{2}$ & $39,2 \%$ & No notificado \\
\cline { 2 - 4 } & $\geq 25 \mathrm{a} 30 \mathrm{Kg} / \mathrm{m}^{2}$ & $40,5 \%$ & No notificado \\
\cline { 2 - 4 } & $\geq 30 \mathrm{Kg} / \mathrm{m}^{2}$ & $20,3 \%$ & No notificado \\
\hline \multirow{2}{*}{$\begin{array}{c}\text { Riesgo anestésico } \\
\text { (escala ASA) }\end{array}$} & $\leq \mathrm{II}$ & $62,2 \%$ & $57,3 \%$ \\
\cline { 2 - 4 } & $\geq \mathrm{III}$ & $37,8 \%$ & $42,7 \%$ \\
\hline $\begin{array}{c}\text { Enfermedad } \\
\text { cardiovascular }\end{array}$ & $\mathrm{No}$ & $55,4 \%$ & $40,5 \%$ \\
\cline { 2 - 4 } & $\mathrm{Si}$ & $44,6 \%$ & $59,5 \%$ \\
\hline \multirow{2}{*}{$\begin{array}{c}\text { Enfermedad } \\
\text { respiratoria }\end{array}$} & $\mathrm{No}$ & $81,1 \%$ & $86,4 \%$ \\
\cline { 2 - 4 } & $\mathrm{Si}$ & $18,9 \%$ & $13,6 \%$ \\
\hline
\end{tabular}

En la tabla 28 se muestra la distribución de los pacientes una vez aplicada la escala PREPARE y Wiltberger en este SDPC en las tres categorías de riesgo, junto con los datos publicados por Uzunoglu et al. (Datos correspondientes a la cohorte prospectiva de validación de la escala, que incluye 429 pacientes) (136) y Wiltberger et al. (137). En ella se puede observar que, en nuestros propios resultados, el porcentaje de pacientes que tenían un riesgo alto es superior en la escala PREPARE que en la escala Wiltberger. 


\begin{tabular}{|c|c|c|c|c|}
\hline \multirow[t]{2}{*}{$\begin{array}{l}\text { CATEGORÍAS } \\
\text { DEL PREPARE/ } \\
\text { WILTBERGER }\end{array}$} & \multicolumn{2}{|c|}{$\begin{array}{c}\text { PACIENTES } \\
\text { SDPC }\end{array}$} & \multirow{2}{*}{$\begin{array}{c}\text { PACIENTES en } \\
\text { la serie de } \\
\text { Wiltberger } \text { et } \text { al. } \\
\text { Escala } \\
\text { Wiltberger }\end{array}$} & \multirow{2}{*}{$\begin{array}{c}\text { PACIENTES } \\
\text { en la serie de } \\
\text { Uzunoglu } e t \\
\text { al. } \\
\text { Escala } \\
\text { PREPARE }\end{array}$} \\
\hline & $\begin{array}{c}\text { Escala } \\
\text { Wiltberger }\end{array}$ & $\begin{array}{c}\text { Escala } \\
\text { PREPARE }\end{array}$ & & \\
\hline Riesgo bajo & $44,6 \%$ & $44,6 \%$ & No notificado & $69,3 \%$ \\
\hline Riesgo medio & $41,9 \%$ & $37,8 \%$ & No notificado & $19,7 \%$ \\
\hline Riesgo alto & $13,5 \%$ & $17,6 \%$ & No notificado & $11 \%$ \\
\hline
\end{tabular}

En cuanto al análisis de los resultados intraoperatorios en el SDPC, la trasfusión de concentrados de hematíes se utilizó en el $11 \%$ de los casos. Este porcentaje es claramente superior al notificado en la SG $(8,6 \%)$. Esto puede ser debido al grado complejidad mayor que supone una DPC comparada con el resto de resecciones pancreáticas. El abordaje laparotómico se realizó en la totalidad de los casos. En un estudio recientemente publicado, comparó la DPC laparoscópica frente a la DPC laparotómica, solo en el $17 \%$ de los pacientes incluidos fueron intervenidos por laparoscopia o cirugía robótica (173)

En la fase de reconstrucción, se utilizaron dos técnicas diferentes fundamentalmente: en el 8,1\% de los pacientes se utilizó reconstrucción en Y de roux, en el 91,9\% restante se prefirió realizar una reconstrucción según técnica de Child, de estos, en el $8,1 \%$ se asoció anastomosis de Braun.

Según un metanálisis recientemente publicado, la reconstrucción tipo Child asociada a una enteroenterostomía de Braun disminuye el RVG clínicamente relevante, 
la FPPO y la fístula biliar. Sin embargo, se necesitan más ensayos clínicos aleatorizados para confirmar esta idea (174).

El drenaje intraabdominal al terminar la cirugía se utilizó en el $86,5 \%$ de los pacientes, porcentaje ligeramente superior a notificado en la SG.

La estancia en la reanimación postquirúrgica de los pacientes incluidos en el SDPC fue similar a los de la SG, debido a que la mayoría de enfermos que pernoctaron en la reanimación postquirúrgica fueron pacientes a los que se les había realizado DPC.

El paciente que no había conseguido tolerancia durante el postoperatorio que ya se había analizado en el anterior apartado de la SG, corresponde a un paciente al que se le había realizado una DPC ya que se ha incluido de nuevo en este análisis. La razón de esto es porque el paciente murió en el postoperatorio con anterioridad a iniciar tolerancia oral.

La comparación de este SDPC en cuanto al tiempo en conseguir la tolerancia de los enfermos en la SG es exactamente la misma. Debido a que, en el SDPC, todos los enfermos tienen al menos una anastomosis intestinal, una biliar y una pancreática, la NPT se pautó como tratamiento en un porcentaje superior de casos $(93,2 \%)$ que los pacientes de la SG (85,5\%). Sin embargo, como se ha descrito previamente, gracias a la implementación cada vez más frecuente de la cirugía mínimamente invasiva y los programas de rehabilitación multimodal en cirugía pancreática, hacen que el uso de NPT, la estancia en la unidad de reanimación postquirúrgica y el tiempo prolongado del drenaje 
intraabdominal sea cada vez menor, como ya se ha descrito ampliamente en la literatura reciente (175-177).

Las complicaciones globales en el SDPC ocurrieron con una frecuencia sutilmente mayor a los analizados en la SG. En este caso, el 78,4\% de los enfermos tuvo algún tipo de complicación, probablemente debido a la mayor complejidad técnica. Además, la recogida de las complicaciones en nuestro estudio fue muy exhaustiva, razón por la cuál no se ha perdido ningún dato y la cifra de morbilidad global puede parecer elevada.

En el gráfico 31 se muestra la comparación de la SG y el SDPC con la literatura médica actual $(130,136,137,152,153,156,160-162)$. El trabajo que tiene una morbilidad más parecida a la notificada en el SDPC de este análisis, es el publicado por el grupo de Sánchez-Cabús et al. (156) que publicaron un 77,5\% de complicación global. Sin embargo, la gran mayoría de ellos (86\%) correspondieron a complicaciones entre los grados I y III de la escala de Dindo et al. (114). 
Gráfico 31: Comparación de nuestros propios resultados con la literatura en cuanto a la tasa de complicaciones globales en pacientes intervenidos de DPC $(129,130,137,152,153,156,160,162)$.

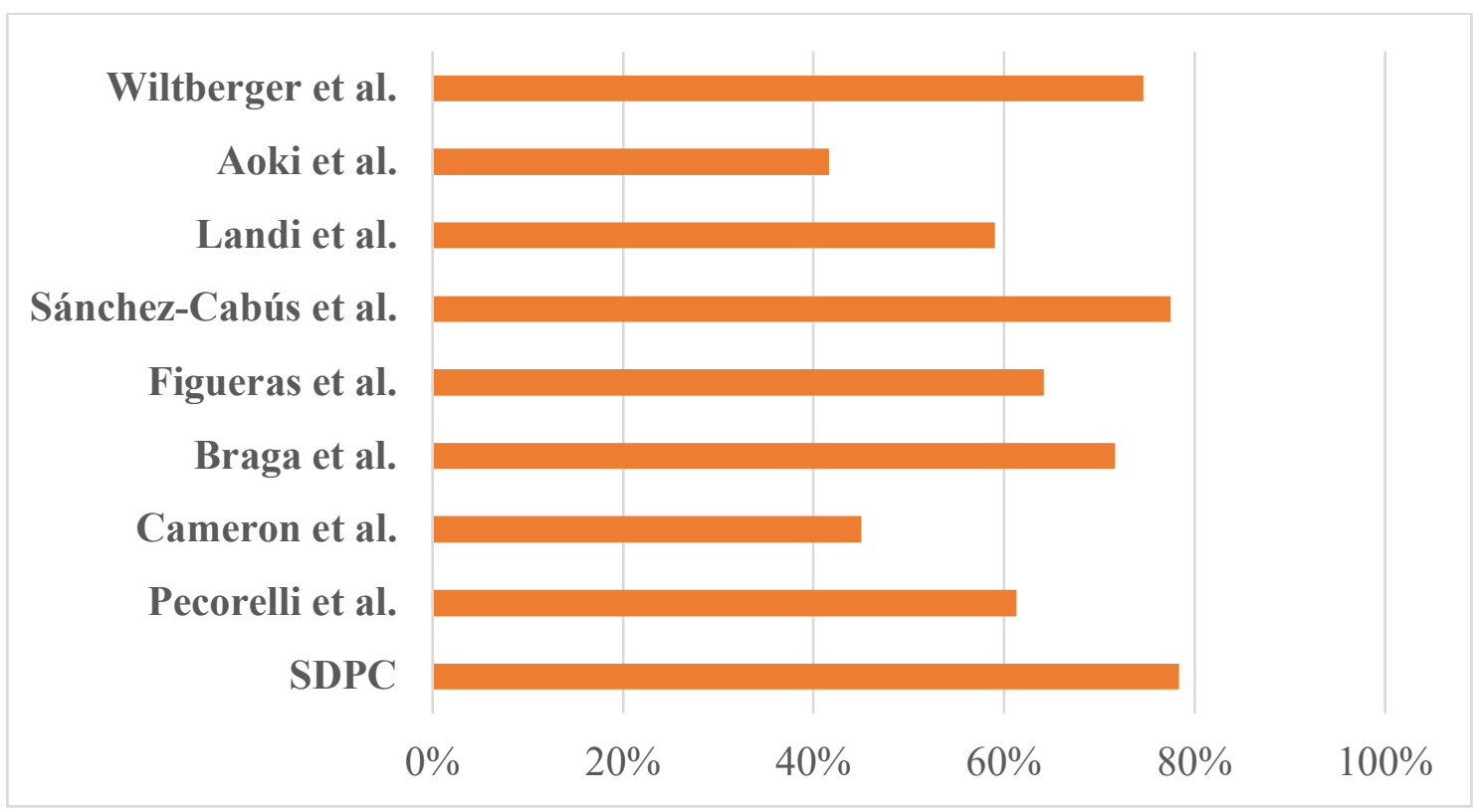

Si se analizan de manera desglosada las complicaciones de los pacientes del SDPC, se puede observar que el 32,4\% de los pacientes tuvieron complicaciones Clavien $\geq$ IIIa, que es considerado como complicación grave según el artículo original que desarrolló la escala PREPARE. Además, este porcentaje es prácticamente igual al notificado por Uzunoglu et al. (136) que fue 33\%. Por otra parte, Celik et al. (139) informó un porcentaje menor, 28,7\%. Comparando esta cifra de complicaciones Clavien $\geq$ IIIa, con las notificados en este mismo trabajo en la SG, destaca que en el SDPC este porcentaje es mayor, esto puede ser debido a la complejidad técnica mayor que supone la DPC con respecto al resto de resecciones pancreáticas mayores. Otra razón para justificar la diferencia en la cifra de complicaciones Clavien $\geq$ IIIa en el SDPC, es que el porcentaje de pacientes que tenían un riesgo alto en la escala $P R E P A R E$ para sufrir complicaciones fue mayor en el SDPC que en la SG (17,6\% frente 13,4\%). 
Según el artículo original que desarrolló la escala de Wiltberger (137), la definición de complicación grave fue un Clavien $\geq$ IIIb durante el postoperatorio. Teniendo en cuenta el grado de complicaciones Clavien $\geq$ IIIb en el SDPC, hemos notificado un 20,3\% de pacientes que reunían este criterio. Por otra parte, Wiltberger et al. (137) publicó que un 29,1\% de sus pacientes desarrollaron complicaciones graves.

Se puede concluir que a pesar de que el porcentaje de complicación global $(78,4 \%)$ pueda parecer excesivo en el SDPC comparado con otras grandes series, el $46 \%$ del total de complicaciones fueron complicaciones Clavien I y II.

Cabe destacar que el 6,8\% de pacientes fue Clavien V, este dato coincide en cifras numéricas con los pacientes que murieron durante el postoperatorio en la SG. Por lo tanto, los 5 pacientes que murieron en este trabajo durante el postoperatorio fueron pacientes a los que se les había realizado una DPC. Uzunoglu et al. (136) y Wiltberger et al. (137) informaron un 5,6\% y un 5,7\% respectivamente de mortalidad durante el postoperatorio.

A la luz de estos datos de morbimortalidad obtenidos en el SDPC, y tras compararlos con los publicados por los autores de las escalas, se puede confirmar que estos resultados tras resección pancreática en términos de complicaciones, son equiparables a los obtenidos en los grandes centros de referencia europeos, a pesar de un volumen inferior de enfermos con patología quirúrgica del páncreas.

En el gráfico 32 está desglosada la distribución del grado de complicaciones según la escala de Dindo et al. (114) y comparada con el trabajo original de Wiltberger et al. (137). 
Los pacientes analizados en el SDPC tuvieron más complicaciones Clavien IV y V comparándolos con los pacientes de la serie de Wiltberger et al. (137). En este gráfico, al igual que ocurre en el gráfico 26, llama especialmente la atención la diferencia que existe en el grado IIIb. De nuevo, la posible explicación a esta desproporción es que, en nuestro análisis, la mayoría pacientes que han sido reintervenidos han tenido también fallo de órgano precisando de esta manera ingreso en la unidad de reanimación postquirúrgica. Por otra parte, existe también una desproporción entre los pacientes incluidos en el grado IIIa. Esto puede ser debido a que la radiología intervencionista y la endoscopia bajo sedación es muy utilizada en los pacientes intervenidos por nuestra unidad, siempre y cuando las complicaciones no suponen una inestabilidad hemodinámica. Wiltberger et al. desgraciadamente, no discuten la diferencia tan abrupta que obtuvieron entre el grado Clavien IIIa y IIIb.

Gráfico 32.: Comparación de nuestros propios resultados con la literatura en cuanto al grado de complicaciones según la escala de Dindo et al. (114) en pacientes intervenidos de DPC.

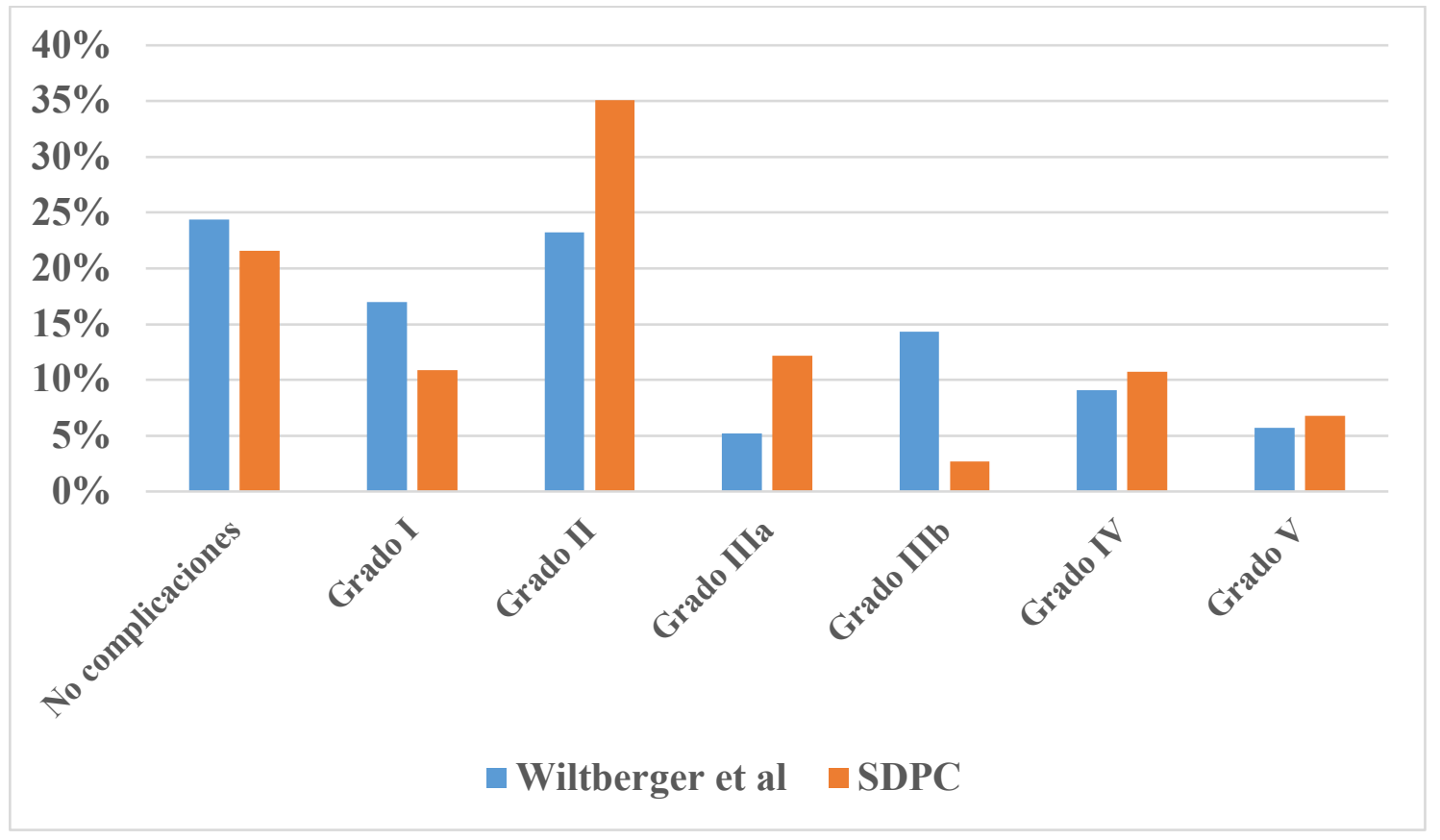


A continuación, los gráficos 33, 34 y 35 comparan los resultados de las complicaciones específicas obtenidas en el SDPC (FPPO, RVG Y HPP) con diversos artículos recientes. Además, en el gráfico de FPPO, se compara la tasa de fístula pancreática clínicamente relevante (FPCR).

La tasa de FPPO en el SDPC es menor que la notificada en este mismo trabajo en la SG. Sin embargo, aunque los pacientes que desarrollan FPPO sean menos que en la SG, el grado de gravedad es mayor, teniendo, por lo tanto, mayor tasa de FPCR con respecto a la SG. En cuanto la comparación de los resultados del SDPC con los datos presentados por el grupo de Wiltberger et al. (137) la tasa de FPPO y FPCR es superior en el SDPC. Otros grandes equipos nacionales e internacionales notificaron tasas de FPCR superiores a la de nuestra unidad $(130,156,159,162)$.

Gráfico 33: Comparación de nuestros propios resultados con la literatura en cuanto a la tasa de FPPO y FPCR $(130,131,137,156,159,160,162)$.

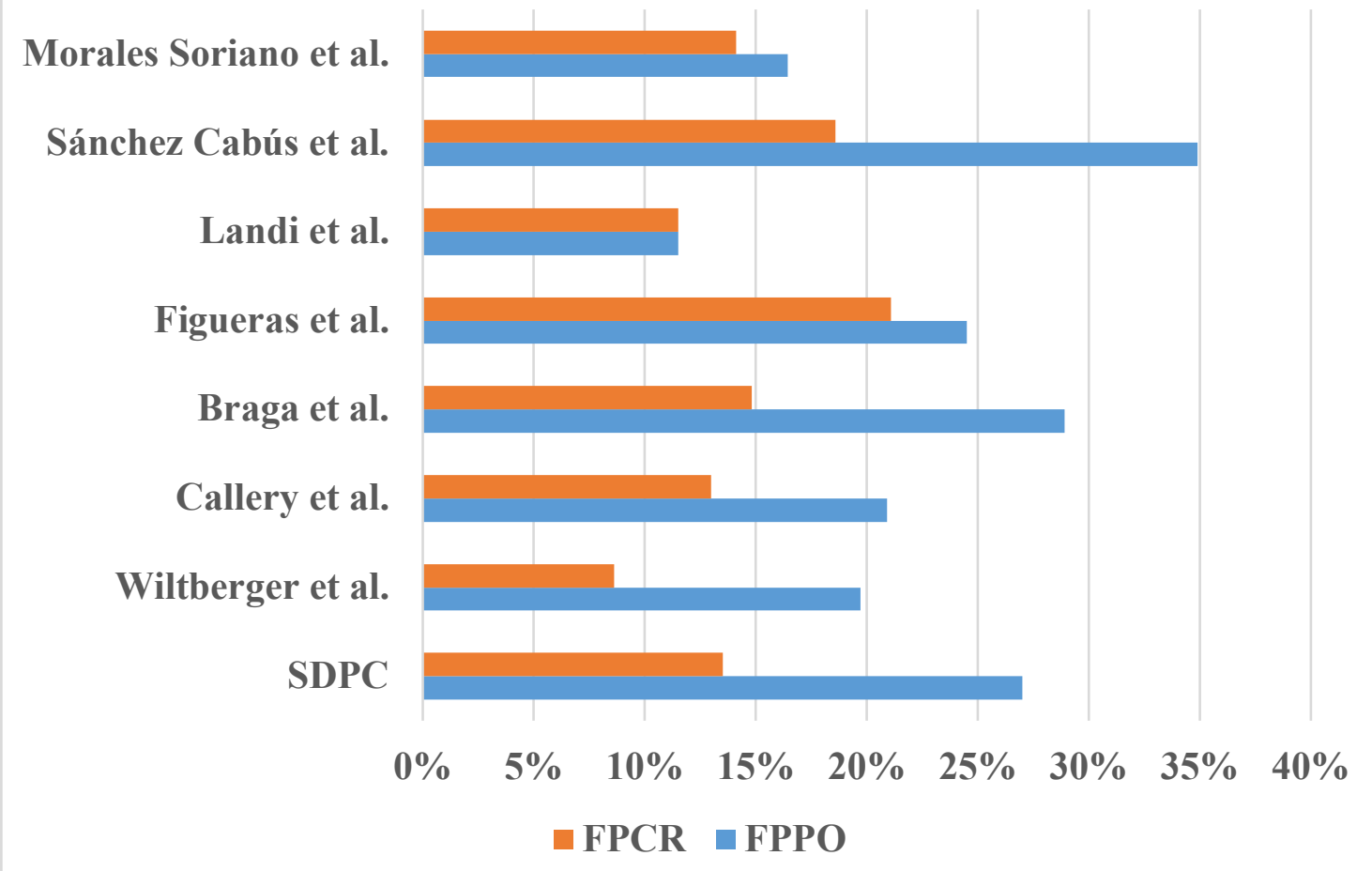


el RVG es una de las complicaciones más comunes tras cirugía pancreática cuya incidencia se sitúa entre el 19\%-57\% (121-123). En el SDPC los pacientes que tuvieron RVG fue superior a la tasa presentada en la SG (33,8\% frente a 26,8\%). Existen grupos que defienden la DPC con preservación pilórica (164). Recientemente, Seis metanálisis han puesto de manifiesto que la DPC con resección pilórica es la técnica requerida para mejorar mencionada complicación $(59,64-68)$.

Gráfico 34: Comparación de nuestros propios resultados con la literatura en cuanto a la tasa de RVG $(130,137,152,156,160,162,164,168)$.

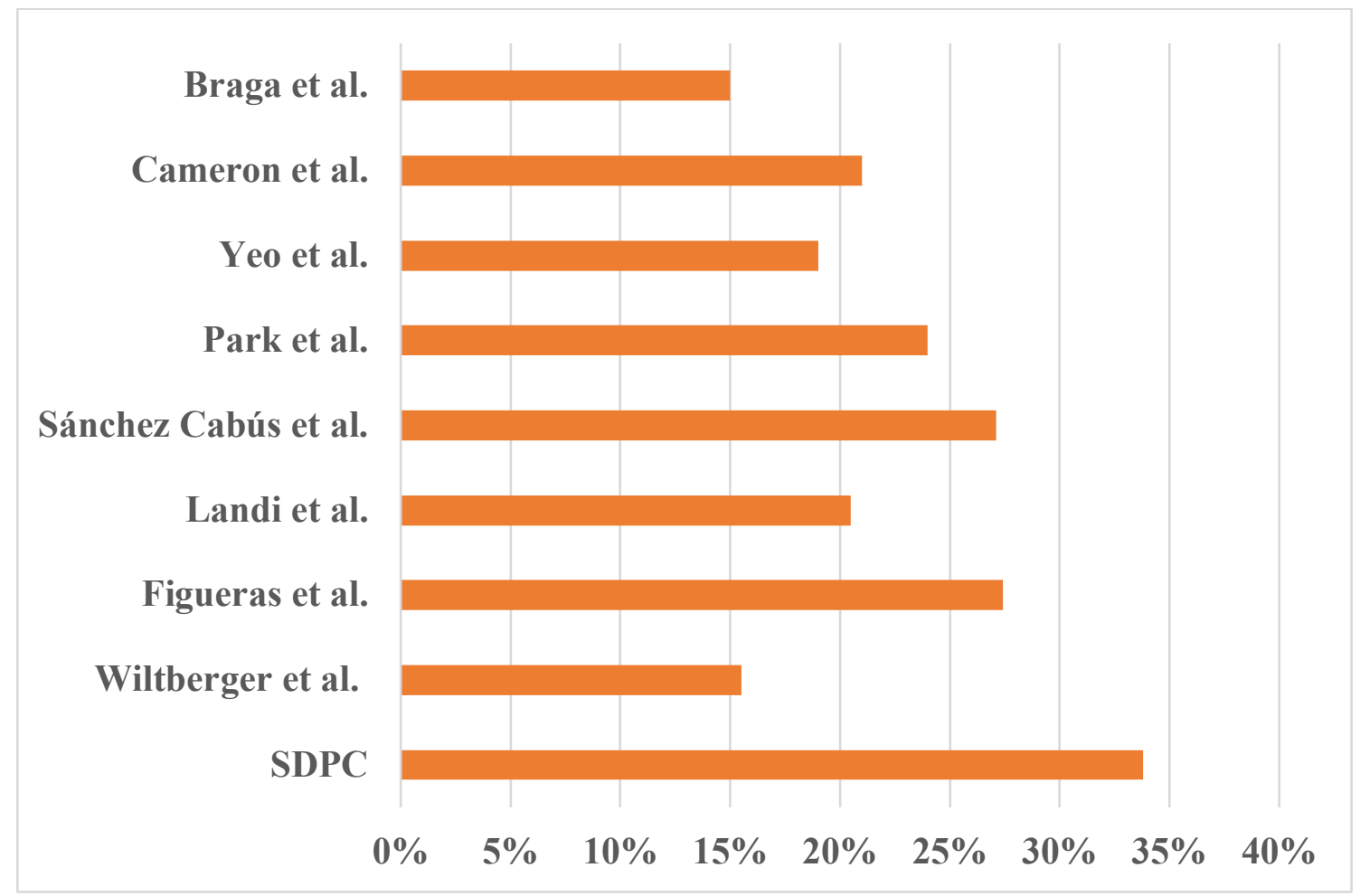

La tasa de HPP en el SDPC es superior al resto de series revisadas, destaca un $23 \%$ de los pacientes que tuvieron HPP, de los cuales el 17,6\% fue HPP grado B y C. Sin embargo, la tasa de complicaciones graves no es mayor como ya se ha descrito 
previamente. Esto es debido a que los grados A y B de HPP suma un 13,5\% de esta tasa, y la condición clínica del enfermo en estos grupos normalmente es buena.

Gráfico 35: Comparación de nuestros propios resultados con la literatura en cuanto a la tasa de HPP $(130,136,137,156,157,160,162,165,166)$.

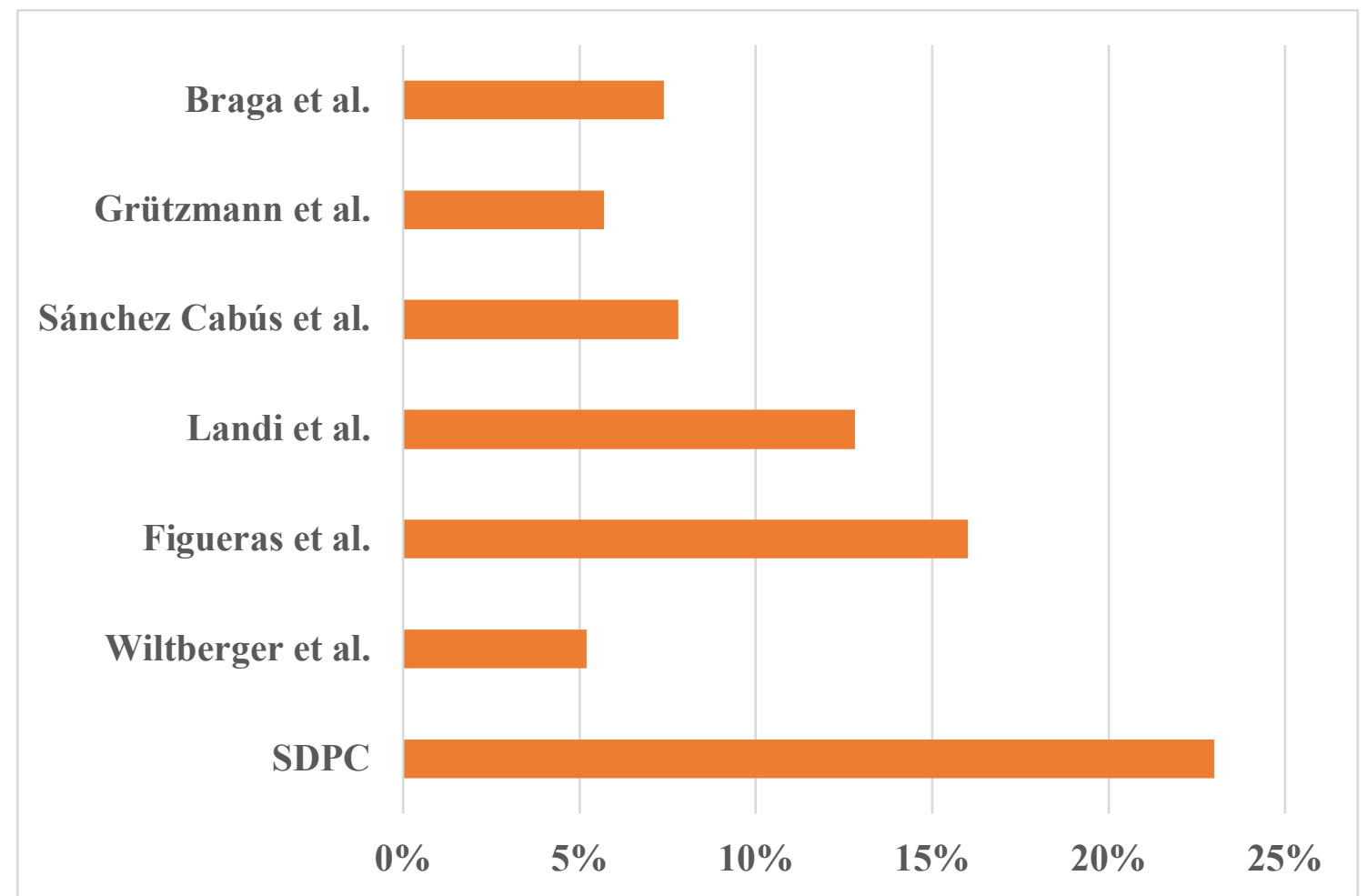

La tasa de IHQ en el SDPC fue 21,6\% de los pacientes, tasa superior a la SG. Este porcentaje también es superior al resto de series revisadas $(129,133)$. Esta cifra hace aumentar la tasa de nuestras complicaciones leves.

En el $10,8 \%$ fue necesaria la reintervención, este porcentaje es superior al notificado en este mismo trabajo en la SG (8,2\%). Sin embargo, es similar al de otras series de grupos españoles $(156,157,159-161,169)$. 
La mediana de estancia postoperatoria global en el SDPC fue de 11 días y RIQ de 9-15 días. En este caso esta cifra coincide con la anteriormente descrita en la SG. En la tabla 29 puede observarse la comparación con el resto de series revisadas en la literatura científica, observando que existe un rango amplio.

\begin{tabular}{|c|c|}
\hline Callery et al. & Media de 8 días. \\
\hline Yeo et al. & Mediana de 13 días. \\
\hline Figueras et al. & Mediana de 14 días. \\
\hline Herrera-cabezón et al. & Mediana de 17 días. \\
\hline Rubio-Manzanares Dorado et al. & Mediana de 18 días. \\
\hline Landi et al. & Mediana de 19 días. \\
\hline Sánchez Cabús et al. & Mediana de 24 días. \\
\hline Morales Soriano et al. & Mediana de 16 días. \\
\hline SDPC. & Mediana de 11 días. \\
\hline SG. & Mediana de 11 días. \\
\hline
\end{tabular}

De igual manera, tal y como se ha realizado en el apartado de la SG, se ha querido evaluar si el análisis de los resultados de la SDPC cumple criterios de calidad. Para ello, se ha utilizado nuevamente los criterios de Sabater et al. (170). [Tabla 30]. 
Tabla 30: comparación de los resultados de los indicadores de calidad entre el artículo de revisión de Sabater et al. (170) y nuestros propios resultados en el SDPC.

\begin{tabular}{|c|c|c|c|}
\hline & $\begin{array}{c}\text { Media de todos los } \\
\text { trabajos revisados }\end{array}$ & SDPC & Límite de calidad \\
\hline Morbilidad global & $58 \%$ & $78,4 \%$ & $<73 \%$ \\
\hline Mortalidad & $4 \%$ & $6,8 \%$ & $<10 \%$ \\
\hline Tasa de FPPO & $18 \%$ & $27 \%$ & $<29 \%$ \\
\hline Tasa de HPP & $11 \%$ & $23 \%$ & $<21 \%$ \\
\hline Reintervención & $11 \%$ & $10,8 \%$ & $<21$ días \\
\hline $\begin{array}{c}\text { Estancia } \\
\text { postoperatoria } \\
\text { global }\end{array}$ & Mediana de 18 días & Mediana de 11 días & \\
\hline
\end{tabular}

El SDPC cumple con amplios márgenes los criterios de calidad en cuanto a mortalidad, FPPO reintervención y estancia postoperatoria global. Sin embargo, la tasa de morbilidad global y la de HPP es superior al límite de calidad aceptado por Sabater et al. (170). Como se ha argumentado previamente, a pesar de que la tasa de morbilidad global pueda considerarse excesiva, los datos de este trabajo se han recogido de manera prospectiva y muy estrictamente, tanto es así, que no se ha notificado ninguna pérdida de ninguna variable en ningún paciente. Es por ello, que cualquier desviación mínima del postoperatorio considerado como "normal" se ha detectado y ha contabilizado como complicación global. 


\subsection{2.- ANÁLISIS DE LA VALIDACIÓN DE LA ESCALA PREPARE Y}

\section{WILTBERGER EN EL SDPC}

La escala PREPARE como se ha indiciado repetidamente, fue desarrollada para predecir complicaciones graves, Clavien $\geq$ IIIa en cualquier resección pancreática mayor (136). En este trabajo, ya se ha validado en la SG. Sin embargo, el objetivo de volverla a utilizar en el SDPC es compararla con la escala Wiltberger que fue validada para pacientes a los que se les va a realizar una DPC exclusivamente. Las características fundamentales de esta segunda escala son las siguientes (137):

- Incluye 4 variables preoperatorias: la presencia de enfermedad cardiovascular, de enfermedad, el grado en la escala de riesgo anestésico ASA, y el IMC. En función de los datos del paciente, se asigna o no una puntuación concreta por cada una de las variables [tabla 13 del apartado Introducción].

- Todos los parámetros que conforman la puntuación total son parámetros clínicos. Esto aporta una ventaja de sencillez en el momento de recoger las variables necesarias.

- Empleo, también en este índice, de la clasificación de Dindo-Clavien (114) para el registro de complicaciones. Sin embargo, a diferencia de la escala PREPARE, la escala de Wiltberger define las complicaciones mayores con una puntuación de Clavien $\geq$ IIIb. Este detalle ha sido tenido en cuenta en nuestro trabajo.

- Así mismo, en la escala de Wiltberger se obtienen las variables antes de realizar la cirugía. De acuerdo a la puntuación obtenida se asigna una categorización de riesgo: bajo, medio y alto [Figura 11]. 
- Fueron reclutados un total de 405 pacientes que iban a ser sometidos a DPC y el análisis de los mismos fue retrospectivo.

- Los datos estadísticos del test son: AUC: 0,83, por lo que según concluyen los autores se trata de un buen test capaz de predecir la aparición de complicaciones Clavien graves ( $\geq$ IIIb).

Una de las novedades fundamentales que aporta nuestro trabajo es precisamente realizar una validación externa de la escala Wiltberger, ni tampoco se ha realizado una comparación entre estas dos escalas previamente.

En la tabla 31 se observan las complicaciones graves que han desarrollado posteriormente los pacientes del SDPC con los datos publicados por Wiltberger et al. (137) y Uzunoglu et al. (136). Se debe recordar nuevamente, ya que es una diferencia fundamental, que la escala de Wiltberger predice complicaciones Clavien $\geq$ IIIb mientras que la escala PREPARE lo hace para complicaciones Clavien $\geq$ IIIa.

Tabla 31: Comparación del porcentaje de complicaciones graves que han desarrollado los pacientes del SDPC respecto a los datos publicados por Wiltberger et al. (137) y Uzunoglu et al. (136), atendiendo a la diferente definición de complicación grave que presenta cada escala.

\begin{tabular}{|c|c|c|c|c|}
\hline \multirow{2}{*}{$\begin{array}{l}\text { CATEGORÍAS } \\
\text { DEL PREPARE/ } \\
\text { WILTBERGER }\end{array}$} & \multicolumn{4}{|c|}{ COMPLICACIONES GRAVES } \\
\hline & $\begin{array}{c}\text { Uzunoglu et al } \\
\text { PREPARE } \\
(136) \\
\text { Clavien } \geq \text { IIIa }\end{array}$ & $\begin{array}{c}\text { SDPC } \\
\text { PREPARE } \\
\text { Clavien } \geq \text { IIIa }\end{array}$ & $\begin{array}{c}\text { Wiltberger et } \\
\text { al. (137) } \\
\text { Clavien } \geq \\
\text { IIIb }\end{array}$ & $\begin{array}{c}\text { SDPC } \\
\text { Wiltberger } \\
\text { Clavien } \geq \\
\text { IIIb }\end{array}$ \\
\hline Riesgo bajo & $18 \%$ & $24,2 \%$ & $6 \%$ & $6,1 \%$ \\
\hline Riesgo medio & $40 \%$ & $25 \%$ & $32 \%$ & $25,8 \%$ \\
\hline Riesgo alto & $66 \%$ & $69,2 \%$ & $76 \%$ & $50 \%$ \\
\hline
\end{tabular}


La escala de PREPARE en el SDPC logra diferenciar y estratificar a los pacientes en este estudio (las categorías de bajo y alto riesgo acumula el 62,2\%) [tabla 28], los autores que desarrollaron la escala notificaron una proporción del 75,5\% (136) (en el grupo de validación interna de los 429 pacientes).

La escala de Wiltberger, por su parte, diferencia a los pacientes entre las categorías de bajo y alto riesgo con menos exactitud acumulando un 58,1\% de los casos, [tabla 28]. Wiltberger et al. (137) desgraciadamente no reflejaron este dato en su artículo.

Para validar las escalas de riesgo PREPARE y Wiltberger en el SDPC, en términos de discriminación (grado en que el modelo distingue entre individuos en los que ocurre el evento y los que no), se ha realizado, como en la SG, una curva ROC. La discriminación aportada por la escala de PREPARE en el SDPC es ligeramente inferior a la aportada en la SG (con un AUC de 0,65 frente a 0,671) y estas dos a su vez inferior al AUC aportada por el artículo original de Uzunoglu et al. (136). En cuanto a los datos de la curva ROC de la escala de Wiltberger, se ha obtenido una cifra más favorable con un AUC de 0,738 dato. Sin embargo, este mismo dato aportado por Wiltberger et al.(137) en su artículo original es mayor con un AUC de 0,83 .

Para completar la validación estadística se realizó un test de calibración empleando el test de bondad de ajuste de Hosmer-Lemeshow. De igual manera a lo descrito en el apartado de la SG, los datos también aquí son favorables ya que una $p>0,05$ hace que las diferencias esperadas y observadas no sean significativas, por lo que la predicción es correcta. La tabla 32 muestra la comparación de datos relativos a la 
validación de la escala PREPARE y Wiltberger en los resultados propios del SDPC, Uzunoglu et al. (136) y Wiltberger et al.

\begin{tabular}{|c|c|c|c|c|}
\hline \multicolumn{5}{|c|}{$\begin{array}{l}\text { Tabla 32: Comparación de los datos estadísticos relativos a la validación de la } \\
\text { escala PREPARE y Wiltberger en la SDPC frente a las series de Uzunoglu et al. } \\
\text { (136) y Wiltberger et al. (137). }\end{array}$} \\
\hline $\begin{array}{c}\text { DATOS } \\
\text { ESTADÍSTICOS }\end{array}$ & $\begin{array}{l}\text { AUC en la } \\
\text { curva ROC }\end{array}$ & $\begin{array}{l}\text { Proporción en } \\
\text { las categorías } \\
\text { de bajo y alto } \\
\text { riesgo }\end{array}$ & $\begin{array}{c}\text { Test de } \\
\text { Hosmer- } \\
\text { Lemeshow }\end{array}$ & $\begin{array}{l}\text { Porcentaje } \\
\text { pronosticado } \\
\text { correcto }\end{array}$ \\
\hline $\begin{array}{l}\text { RESULTADOS } \\
\text { PROPIOS } \\
\text { PREPARE } \\
\text { SDPC }\end{array}$ & $\begin{array}{c}0,65 \\
(\mathrm{IC} 95 \%= \\
0,508-0,793 \\
\mathrm{p}=0,0037)\end{array}$ & $\begin{array}{l}62,2 \% \text { de los } \\
\text { pacientes de } \\
\text { la muestra }\end{array}$ & $\mathrm{p}=0,095$ & $74,3 \%$ \\
\hline UZUNOGLU et al. * & $\begin{array}{c}0,709 \\
(\mathrm{IC} 95 \%= \\
0,657-0,760 \\
\mathrm{p}=0,001)\end{array}$ & $\begin{array}{c}75,5 \% \text { de los } \\
\text { pacientes de la } \\
\text { muestra }\end{array}$ & $\mathrm{p}=0,454$ & $75 \%$ \\
\hline $\begin{array}{c}\text { RESULTADOS } \\
\text { PROPIOS } \\
\text { WILTBERGER } \\
\text { SDPC } \\
\end{array}$ & $\begin{array}{c}0,738 \\
(\mathrm{IC} 95 \% 0,6 \\
-0,876, \\
\mathrm{p}=0,005)\end{array}$ & $\begin{array}{c}58,1 \% \text { de los } \\
\text { pacientes de la } \\
\text { muestra }\end{array}$ & $\mathrm{p}=0,619$ & $79,7 \%$ \\
\hline WILTBERGER et al. & $\begin{array}{c}0,83 \\
(\mathrm{IC} 95 \%= \\
0,75-0,89)\end{array}$ & No notificado & $\mathrm{p}=0,98$ & $\begin{array}{c}\text { No } \\
\text { notificado }\end{array}$ \\
\hline
\end{tabular}

* Datos correspondientes a la cohorte prospectiva de validación de la escala, que incluye 429 pacientes.

\subsection{3.- ANÁLISIS BIVARIANTE ENTRE ESCALA PREPARE}

\section{WILTBERGER EN EL SDPC Y LAS VARIABLES RESULTADO}

La escala de Wiltberger de la misma manera que la escala PREPARE no fueron descrita para asociarse a complicaciones globales, complicaciones específicas postpancreatectomía, reintervención, mortalidad postoperatoria ni IHQ. De nuevo, en el SDPC se ha querido relacionar si los grados de estadificación de riesgo prequirúrgicos se asocian a la aparición de alguna de estas variables. 
Los niveles de riesgo de la escala PREPARE se han asociado de manera estadísticamente significativa a complicaciones globales, reintervención quirúrgica y exitus tal y como también se asociaron en la SG.

Los grados de riesgo de la escala Wiltberger se asociaron de manera estadísticamente significativa a reintervención y a todas las complicaciones específicas postpancreatectomía (FPPO, RVG y HPP). Aunque no ha sido estudiada anteriormente la escala Wiltberger como escala pronóstica de complicaciones específicas postpancreatectomía, esto podría ser una futura línea de investigación tras observar estos resultados, aunque debido al escaso tamaño muestral y a la ausencia de análisis multivariante, no podemos realizar esta afirmación.

Finalmente cabe señalar que la única variable resultado que se ha asociado a los grados de riesgo de la escala PREPARE tanto en la SG y SDPC y los grados de riesgo de la escala Wiltberger es la reintervención. Esto parece lógico si se analiza detalladamente, ya que la definición de complicación grave según Wiltberger et al. (137) (Clavien $\geq$ IIIb), supone reintervención asociado o no a fallo de órgano o incluso exitus en un último caso

\section{3.- INTEGRACIÓN Y APLICABILIDAD FUTURA.}

Este estudio analiza una serie de pacientes sometidos a cirugía pancreática y registrados en una base de datos prospectiva. Hasta la fecha, no nos consta ningún grupo más en España que haya validado la escala PREPARE ni Wiltberger. 
Se considera que, aunque el tamaño muestral alcanzado es aún escaso comparado con otras grandes series, los pacientes presentan una distribución demográfica similar a los datos publicados en la literatura, al igual que los resultados postquirúrgicos, en términos de complicaciones graves (grados III, IV y V de la clasificación de DindoClavien), como hemos observado y analizado en nuestro estudio.

En base a los resultados obtenidos en los test estadísticos de validación, se puede catalogar que el PREPARE es una escala fiable para predecir el riesgo de complicaciones graves tras la cirugía de páncreas, al tiempo que útil para realizar comparaciones de tasas de morbimortalidad respecto a otros equipos de cirujanos, ya que estratifica a los pacientes según sus características clínicas basales, según lo obtenido en la SG en este trabajo. El porcentaje pronosticado correcto de este trabajo es superior a la validación interna publicada en artículo de Uzunoglu et al. (136) y al aportado por Celik et al. (139).

En el SDPC, No obstante, Aunque la escala PREPARE y Wiltberger son fiables pronosticando complicaciones graves, la escala Wiltberger ha obtenido mejores resultados en los mencionados test estadísticos, ya que el AUC de la curva ROC, y el porcentaje pronosticado correcto son mejores que en la escala PREPARE.

Una de las razones por la cual la escala Wiltberger ha sido mejor considerada en nuestro trabajo puede ser la siguiente: al calcular los puntos de la escala PREPARE, la variable que más puntos otorga es la albúmina (5 puntos). En segundo lugar, la variable que más puntos otorga es el tipo de cirugía (urgente 4 puntos). En este trabajo, todos los pacientes han sido operados de manera programada, no se ha realizado ninguna resección pancreática de urgencia, por lo que estos 4 puntos no han sido sumados en ninguno de los 
pacientes de este trabajo. Otra variable cuyos puntos son sumados a todos los pacientes del SDPC es la técnica quirúrgica, ya que todos los pacientes de esta serie se les ha realizado DPC por lo que todos parten con 2 puntos de entrada.

Otra diferencia fundamental entre ambas escalas, es como se ha descrito previamente, la diferente definición de complicación grave, la escala PREPARE considera esta como Clavien $\geq$ IIIa y Wiltberger como Clavien $\geq$ IIIb. Esto puede generar heterogeneidad de resultados al comparar estas distintas escalas en una misma muestra de pacientes.

Se ha descrito también en este trabajo que existen otras muchas escalas pronósticas de morbimortalidad postquirúrgica tras este tipo de cirugía. Esto es así porque la información de la probabilidad de complicación postquirúrgica de un paciente al que todavía no ha sido sometido a cirugía, puede hacer que se adecuen más medidas de seguridad e individualizar el tratamiento en función de su riesgo. Entre la abundante existencia de escalas que han sido publicadas en la literatura, no existe ninguna que se haya implementado de manera universal.

Uno de los posibles trabajos para líneas futuras, sería realizar una comparación múltiple con un tamaño muestral grande entre todas las escalas con el objetivo de determinar qué variables son realmente importantes y que escalas son efectivas. Probablemente, no existe una única escala totalmente efectiva, sino que puede ser necesaria la integración de muchas escalas con las variables que influyen más desde el punto de vista pronóstico durante el postoperatorio. 


\section{4.- LIMITACIONES}

- La primera de ellas es el bajo tamaño muestral alcanzado y la realización del estudio en un único centro hospitalario, lo que limita la extrapolación y generalización de resultados a una población más amplia de pacientes sometidos a cirugía de páncreas.

- Excluyendo a los pacientes a los que se realizó DPT, que no se midió la amilasa en el líquido de drenaje, existen pocos pacientes a los que se realizó DPC en los que no se utilizó drenaje intraabdominal, (Todos los pacientes a los que se realizó PD se dejo drenaje intraabdominal al finalizar la cirugía). Es por ello, que no se ha podido medir la amilasa en el líquido de drenaje en 10 pacientes los que se realizó DPC. De tal manera, que se ha podido infraestimar la tasa de fístula bioquímica en estos 10 pacientes.

- En nuestro análisis, durante el tiempo de inclusión y la recogida de datos, no hemos podido incluir a ningún paciente en el que se haya realizado una resección pancreática urgente ya que esto es excepcional. En el trabajo de Uzunoglu et al. realizado por centros de referencia en cirugía pancreática, notificaron únicamente un 3,6\% de resecciones pancreáticas urgentes.

- En nuestro trabajo se han excluido a los pacientes a los que se realizó una resección pancreática no prevista en el contexto de otro tipo de cirugía. Esto es debido a que no se pudo medir antes de la intervención quirúrgica las diferentes variables que componen las escalas PREPARE y Wiltberger. Igualmente, creemos importante señalar que aunque se hubiera podido preveer la resección pancreática, otro gesto quirúrgico asociado en otro órgano puede incrementar la morbimortalidad postoperatoria. 


\section{5.- CONTROL DE CALIDAD DE LA VALIDACIÓN EXTERNA}

En este estudio se ha querido evaluar si la validación externa de las escalas PREPARE y Wiltberger se ha realizado de manera correcta. Para ello, se ha utilizado el modelo TRIPOD, cuyo objetivo es asegurar que se cumplen todos los pasos necesarios a la hora de certificar una validación externa de una escala pronóstica o diagnóstica anteriormente desarrollada, asegurado de esta manera que se cumplan los criterios de calidad necesarios. En la tabla 33 puede observarse las recomendaciones del modelo TRIPOD aportadas por Collins et al. (178).

Nuestro estudio cumple los requisitos del modelo TRIPOD para ser considerado como estudio de validación externa de calidad. En este caso, los únicos dos requisitos de los anteriormente expuestos que no fueron considerados son la ausencia de un resumen para el mencionado trabajo y el enmascaramiento de los pacientes.

Este modelo fue desarrollado para evaluar la validación externa de escalas que van a ser publicadas en artículos científicos por lo que este criterio es algo fundamental en ese contexto. En nuestro análisis no se cumple el enmascaramiento de la muestra ya que el mismo investigador que ha recogido las variables fue el que las analizó. El punto 9 informa sobre si se han descrito los datos perdidos, pero en este trabajo no se ha perdido ninguna variable de ningún paciente. 


\begin{tabular}{|c|c|c|c|}
\hline \multicolumn{4}{|l|}{ Título y resumen } \\
\hline Título & 1 & $\begin{array}{l}\text { Identificar el estudio como estudio de validación de un modelo de predicción multivariable, la } \\
\text { población objetivo y el resultado a predecir. }\end{array}$ & \\
\hline Resumen & 2 & $\begin{array}{l}\text { Proporcionar un resumen de objetivos, diseño del estudio, entorno, participantes, tamaño de la } \\
\text { muestra, predictores, resultados, análisis estadístico, resultados y conclusiones. }\end{array}$ & $\mathrm{X}$ \\
\hline \multicolumn{4}{|c|}{ 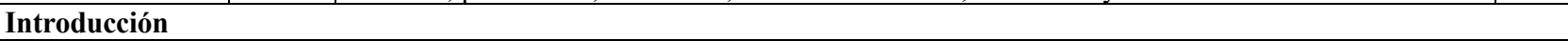 } \\
\hline \multirow[t]{2}{*}{$\begin{array}{l}\text { Introducción y } \\
\text { objetivos }\end{array}$} & $3 a$ & $\begin{array}{l}\text { Explicar el contexto médico (incluso si es diagnóstico o pronóstico) y la justificación para } \\
\text { desarrollar o validar el modelo de predicción multivariable, incluidas las referencias a modelos } \\
\text { existentes. }\end{array}$ & $\sqrt{ }$ \\
\hline & $3 b$ & Especificar los objetivos. & $\sqrt{ }$ \\
\hline \multicolumn{4}{|c|}{ Pacientes y métodos } \\
\hline \multirow{2}{*}{$\begin{array}{l}\text { Información de } \\
\text { los datos }\end{array}$} & $4 \mathrm{a}$ & Describir el diseño del estudio (por ejemplo, ensayo aleatorio, cohortes o registros). & $\sqrt{ }$ \\
\hline & $4 \mathrm{~b}$ & Especificar las fechas en las que se desarrolló el estudio. & $\sqrt{ }$ \\
\hline \multirow[t]{3}{*}{ Participantes } & $5 \mathrm{a}$ & $\begin{array}{l}\text { Especificar los elementos clave del entorno del estudio (por ejemplo, atención primaria, } \\
\text { atención secundaria, población general). }\end{array}$ & \\
\hline & $5 \mathrm{~b}$ & Describir los criterios de inclusión en el estudio. & \\
\hline & $5 \mathrm{c}$ & Dar detalles de los tratamientos recibidos, si son relevantes. & \\
\hline \multirow[t]{2}{*}{ Resultado } & $6 a$ & $\begin{array}{l}\text { Definir claramente el resultado que predice el modelo de predicción, incluido cómo y cuándo } \\
\text { se evalúa. }\end{array}$ & \\
\hline & $6 \mathrm{~b}$ & Informar cualquier acción para evaluar ciegamente el resultado a predecir. & $\mathrm{X}$ \\
\hline \multirow[t]{2}{*}{ Predictores } & $7 \mathrm{a}$ & $\begin{array}{l}\text { Definir claramente todas las variables utilizadas en la escala pronóstica para validar el modelo } \\
\text { de predicción, incluido cómo y cuándo se midieron. }\end{array}$ & \\
\hline & $7 \mathrm{~b}$ & $\begin{array}{l}\text { Informar cualquier acción a la evaluación ciega de predictores para el resultado y otros } \\
\text { predictores. }\end{array}$ & \\
\hline $\begin{array}{l}\text { Tamaño } \\
\text { muestra }\end{array}$ & 8 & Explicar como se llegó al tamaño muestral. & \\
\hline Datos perdidos & 9 & Describir como se manejaron los datos perdidos. (No se ha perdido ningún dato) & \\
\hline \multirow{3}{*}{$\begin{array}{l}\text { Análisis } \\
\text { estadístico }\end{array}$} & $10 \mathrm{c}$ & Para la validación, describir como se calcularon las escalas pronosticas. & \\
\hline & $10 \mathrm{~d}$ & $\begin{array}{l}\text { Especificar todas las medidas utilizadas para evaluar el rendimiento del modelo y, si es } \\
\text { relevante, comparar varios modelos. }\end{array}$ & \\
\hline & $10 \mathrm{e}$ & Describir cualquier actualización del modelo que haya sido validada. & \\
\hline $\begin{array}{l}\text { Grupos de } \\
\text { riesgo }\end{array}$ & 11 & Dar detalles en cómo han sido creados los grupos de riesgo. & \\
\hline Desarrollo & 12 & $\begin{array}{l}\text { Identificar cualquier diferencia con los datos de desarrollo en el entorno, los criterios de } \\
\text { elegibilidad, el resultado y los predictores. }\end{array}$ & \\
\hline \multicolumn{4}{|l|}{ Resultados } \\
\hline \multirow[t]{3}{*}{ Participantes } & $13 \mathrm{a}$ & $\begin{array}{l}\text { Describir el flujo de participantes a través del estudio, incluido el número de participantes con } \\
\text { y sin el resultado y, si corresponde, un resumen del tiempo de seguimiento. }\end{array}$ & \\
\hline & $13 \mathrm{~b}$ & $\begin{array}{l}\text { Describir las características de los participantes (datos demográficos básicos, características } \\
\text { clínicas, predictores disponibles), incluido el número de participantes con datos faltantes para } \\
\text { los predictores y el resultado. }\end{array}$ & \\
\hline & $13 \mathrm{c}$ & $\begin{array}{l}\text { Para la validación, muestre una comparación con los datos del artículo de original de la escala } \\
\text { en cuanto a variables importantes (demografía, predictores y resultados). }\end{array}$ & \\
\hline Desarrollo & 16 & $\begin{array}{l}\text { Realizar pruebas para demostrar la fiabilidad y la calibración de los datos del estudio en el } \\
\text { modelo a validar, mostrando los intervalos de confianza. }\end{array}$ & \\
\hline Actualización & 17 & Si se ha realizado alguna actualización del modelo, informar sobre los datos actualizados. & \\
\hline \multicolumn{4}{|l|}{ Discusión } \\
\hline Limitaciones & 18 & $\begin{array}{l}\text { Discutir sobre cualquier limitación del estudio como (muestra no representativa pocos casos } \\
\text { para predecir, datos perdidos...). }\end{array}$ & \\
\hline \multirow[t]{2}{*}{ Interpretación } & $19 \mathrm{a}$ & $\begin{array}{l}\text { Para la validación, argumentar los resultados referenciándose a los resultados del artículo } \\
\text { original y de otros modelos validados. }\end{array}$ & \\
\hline & $19 \mathrm{~b}$ & $\begin{array}{l}\text { Dar una interpretación global de los resultados considerando los objetivos, limitaciones, } \\
\text { resultados similares de otros estudios y cualquier otra información relevante que se considere. }\end{array}$ & \\
\hline Implicaciones & 20 & $\begin{array}{l}\text { Discutir el potencial uso clínico del modelo y de las implicaciones que este puede tener para } \\
\text { futuras investigaciones. }\end{array}$ & $\sqrt{ }$ \\
\hline
\end{tabular}


Francisco Javier Tejero Pintor 


\section{CONCLUSIONES}


Francisco Javier Tejero Pintor 


\section{6.- CONCLUSIONES}

1.- Los pacientes intervenidos de resección pancreática mayor en el Hospital Universitario Río Hortega de Valladolid fueron predominantemente varones entre la quinta y séptima década de la vida. En la serie global, tres de cada cuatro pacientes sufrieron algún tipo de complicación postquirúrgica, de las cuales el 26\% fueron graves. En el subgrupo de pacientes sometidos a duodenopancreatectomía cefálica, la morbilidad global fue discretamente superior, con un 32\% de complicaciones graves.

2.- En la serie global, según la escala $P R E P A R E$, la mayoría de los pacientes fueron clasificados como bajo riesgo para sufrir complicaciones graves; existiendo un ascenso progresivo y estadísticamente significativo de la incidencia de las mismas en cada categoría de riesgo.

3.- En el subgrupo de pacientes sometidos a duodenopancreatectomía cefálica, según la escala PREPARE, la mayoría de los pacientes fueron clasificados como bajo y medio riesgo para sufrir complicaciones graves; existiendo un ascenso progresivo $\mathrm{y}$ estadísticamente significativo de la incidencia de las mismas en cada categoría de riesgo.

4.- Aplicando la escala Wiltberger en el subgrupo de duodenopancreatectomía cefálica, la mayoría de los pacientes fueron clasificados como bajo y medio riesgo para sufrir complicaciones graves; existiendo un ascenso progresivo y estadísticamente significativo de la incidencia de las mismas en cada categoría de riesgo. 
5.- Tanto la escala PREPARE en la serie global como la escala PREPARE y Wiltberger en el subgrupo de duodenopancreatectomía cefálica resultaron fiables desde el punto de vista estadístico. Ambas obtuvieron buenos resultados en términos de discriminación y calibración. La escala Wiltberger resultó ser ligeramente superior a la escala PREPARE en el subgrupo de duodenopancreatectomía cefálica.

6.- La escala PREPARE se asoció de manera estadísticamente significativa con morbilidad global, reintervención quirúrgica y mortalidad postoperatoria tanto en la serie global como en el subgrupo de duodenopancreatectomía cefálica, y con la fístula pancreática postoperatoria en el subgrupo de duodenopancreatectomía cefálica. Por su parte, la escala Wiltberger mostró una asociación significativa con la reintervención y con las tres complicaciones específicas post-pancreatectomía: hemorragia postoperatoria postpancreatectomía, fistula pancreática postoperatoria y retraso de vaciamiento gástrico.

7.- Este trabajo ha confirmado la utilidad que presentan las escalas PREPARE y Wiltberger para pronosticar el riesgo de complicaciones graves tras resección pancreática mayor. Nuestro grupo ha sido el segundo en realizar una validación externa de la escala PREPARE y el primero en realizar la validación externa de la escala Wiltberger. En los pacientes que van a ser sometidos a duodenopancreatectomía cefálica, la escala Wiltberger es ligeramente más fiable que la escala PREPARE. 


\section{BIBLIOGRAFÍA}


Francisco Javier Tejero Pintor 


\section{7.- BIBLIOGRAFÍA}

1. Tando Y, Yanagimachi M, Matsuhashi Y, Nakamura T, Kamisawa T. A brief outline of the history of the pancreatic anatomy. Dig Surg. 2010;27(2):84-6.

2. Rodriguez-López M, Bailón-Cuadrado M, Tejero-Pintor F, Perez-Saborido B. Estado inmunonutricional y cáncer de páncreas. An Real Acad Med Cir Vall. $2017 ; 54: 27-45$.

3. Schnelldorfer T, Adams DB, Warshaw AL, Lillemoe KD, Sarr MG. Forgotten pioneers of pancreatic surgery: Beyond the favorite few. Ann Surg. 2008;247(1):191-202.

4. Howard JM. History of pancreatic head resection-the evaluation of surgical technique. Am J Surg. 2007;194(4 SUPPL.):6-10.

5. Whipple AO, Parsons WB, Mullins CR. Treatment of carcinoma of the ampulla of Vater. Ann Surg. 1935;102(4):763-79.

6. Whipple AO. Pancreaticoduodenectomy for Islet Carcinoma. Ann Surg. $1945 ; 121(6): 847-52$.

7. Rockey EW. Total pancreatectomy for carcinoma: A case report. Ann Surg. 1943;118(4):603-11.

8. Priestley JT, Comfort MW, Radcliffe J. Total Pancreatectomy for Hyperinsulinism

Due to an Islet-Cell Adenoma: Survival and Cure at Sixteen Months after Operation Presentation of Metabolic Studies. Ann Surg. 1944;119(2):211-21.

9. Basturk O, Hong S, Wood LD, Brosens LAA, Fukushima N, Goggins M, et al. A Revised Classification System and Recommendations From the Baltimore Consensus Meeting for Neoplastic Precursor Lesions in the Pancreas. Ann J Surg Pathol. 2015;39(12):1730-41. 
10. Schaberg KB, Dimaio MA, Longacre TA. Intraductal Papillary Mucinous Neoplasms Often Contain Epithelium From Multiple Subtypes and / or Are Unclassifiable. Am J Surg Pathol. 2016;40(1):44-50.

11. Tanaka M, Castillo CF, Adsay V, Chari S, Falconi M, Jang J, et al. Pancreatology International consensus guidelines 2012 for the management of IPMN and MCN of the pancreas. Pancreatol. 2012;12(3):183-97.

12. Nakata K, Ohuchida K, Aishima S, Sadakari Y, Kayashima T, Miyasaka Y et al. Invasive Carcinoma Derived From Intestinal-Type Intraductal Papillary Mucinous Neoplasm Is Associated With Minimal Invasion, Colloid Carcinoma, and Less Invasive Behavior, Leading to a Better Prognosis. Pancreas. 2011;40(4):581-7.

13. Tanaka M, Fernández-del Castillo C, Kamisawa T, Jang JY, Levy P, Ohtsuka T, et al. Revisions of international consensus Fukuoka guidelines for the management of IPMN of the pancreas. Pancreatology. 2017;17(5):738-53.

14. Barral M, Soyer P, Dohan A, Laurent V, Hoeffel C, Fishman EK, et al. Magnetic resonance imaging of cystic pancreatic lesions in adults: An update in current diagnostic features and management. Abdom Imaging. 2014;39(1):48-65.

15. Scott J, Martin I, Redhead D, Hammond P, Garden OJ. Mucinous cystic neoplasms of the pancreas: Imaging features and diagnostic difficulties. Clin Radiol. 2000;55(3):187-92.

16. Xourafas D, Tavakkoli A, Clancy TE, Ashley SW. Noninvasive intraductal papillary mucinous neoplasms and mucinous cystic neoplasms: Recurrence rates and postoperative imaging follow-up. Surg (United States). 2015;157(3):473-83.

17. Karoumpalis I, Christodoulou DK. Cystic lesions of the pancreas. Ann Gastroenterol. 2016;29(2):155-61.

18. Ketwaroo GA, Mortele KJ, Sawhney MS. Pancreatic Cystic Neoplasms: An 
Update. Gastroenterol Clin North Am. 2016;45(1):67-81.

19. Kimura W, Moriya T, Hanada K, Abe H, Yanagisawa A, Fukushima N, et al. Multicenter study of serous cystic neoplasm of the Japan pancreas society. Pancreas. 2012;41(3):380-7.

20. Reddy S, Cameron JL, Scudiere J, Hruban RH, Fishman EK, Ahuja N, et al. Surgical Management of Solid-Pseudopapillary Neoplasms of the Pancreas (Franz or Hamoudi Tumors): A Large Single-Institutional Series. J Am Coll Surg. 2009;208(5):950-7.

21. Butte JM, Brennan MF, Gönen M, Tang LH, D’Angelica MI, Fong Y, et al. Solid pseudopapillary tumors of the pancreas. clinical features, surgical outcomes, and long-term survival in 45 consecutive patients from a single center. J Gastrointest Surg. 2011;15(2):350-7.

22. Valsangkar NP, Morales-Oyarvide V, Thayer SP, Ferrone CR, Wargo JA, Warshaw AL, et al. 851 resected cystic tumors of the pancreas: A 33-year experience at the Massachusetts General Hospital. Surg (United States). 2012;152(3 SUPPL.):4-12.

23. Lanke G, Ali FS, Lee JH. Clinical update on the management of pseudopapillary tumor of pancreas. World J Gastrointest Endosc. 2018;10(9):145-55.

24. Romics L, Oláh A, Belágyi T, Hajdú N, Gyurus P, Ruszinkó V. Solid pseudopapillary neoplasm of the pancreas-proposed algorithms for diagnosis and surgical treatment. Langenbeck’s Arch Surg. 2010;395(6):747-55.

25. Machado MCC, Machado MAC, Bacchella T, Jukemura J, Almeida JL, Cunha JEM. Solid pseudopapillary neoplasm of the pancreas: distinct patterns of onset, diagnosis, and prognosis for male versus female patients. Surgery. 2008;143(1):29-34. 
26. Yadav D, Timmons L, Benson JT, Dierkhising RA, Chari ST. Incidence, prevalence, and survival of chronic pancreatitis: A population-based study. Am J Gastroenterol. 2011;106(12):2192-9.

27. Bruno MJ. Chronic pancreatitis. Gastrointest Endosc Clin N Am. 2005;15(1):5562.

28. Jemal A, Bray F, Center MM, Ferlay J, Ward E FD. Global Cancer Statistics. Ca Cancer J Clin. 2011;61:69-90.

29. Modolell I, Guarner L, Malagelada JR. Vagaries of clinical presentation of pancreatic and biliary tract cancer. Ann Oncol. 1999;10(Suppl 4):82-4.

30. Allen PJ, Kuk D, Fernandez-del Castillo D, Basturk O, Christopher L., Wolfgang M, et al. Multi-Institutional validation study of the American Joint Commission on Cancer (8th edition) changes for $\mathrm{T}$ and $\mathrm{N}$ staging in patients with pancreatic adenocarcinoma. Ann Surg. 2017;285(1):185-91.

31. Kang MJ, Jang JY, Chang YR, Kwon W, Jung W, Kim SW. Revisiting the concept of lymph node metastases of pancreatic head cancer: Number of metastatic lymph nodes and lymph node ratio according to $\mathrm{N}$ stage. Ann Surg Oncol. 2014;21(5):1545-51.

32. Ryan DP, Hong TS, Bardeesy N. Pancreatic Adenocarcinoma. New Eng J Med. 2014;371:1039-49.

33. Wente MN, Shrikhande S V., Kleeff J, Müller MW, Gutt CN, Büchler MW, et al. Management of early hemorrhage from pancreatic anastomoses after pancreaticoduodenectomy. Dig Surg. 2006;23(4):203-8.

34. Junrungsee S, Kittivarakul E, Ko-iam W, Lapisatepun W, Sandhu T, Chotirosniramit A. Prognostic Factors and Survival of Patients with Carcinoma of the Ampulla of Vater after Pancreaticoduodenectomy. DoiOrg. 2017;18(1):225-9. 
35. Rostain F, Hamza S, Drouillard A, Faivre J, Bouvier AM, Lepage C. Trends in incidence and management of cancer of the ampulla of Vater. World $\mathrm{J}$ Gastroenterol. 2014;20(29):10144-50.

36. Klein F, Jacob D, Bahra M, Pelzer U, Puhl G, Krannich A, et al. Prognostic factors for long-term survival in patients with ampullary carcinoma: The results of a 15year observation period after pancreaticoduodenectomy. HPB Surg. 2014;2014.

37. Riall TS, Cameron JL, Lillemoe KD, Campbell KA, Sauter PK, Coleman J, et al. Pancreaticoduodenectomy with or without distal gastrectomy and extended retroperitoneal lymphadenectomy for periampullary adenocarcinoma - Part 3: Update on 5-year survival. J Gastrointest Surg. 2005;236:355-66.

38. Duffy JP, Hines OJ, Liu JH, Ko CY, Cortina G, Isacoff WH, et al. Improved survival for adenocarcinoma of the ampulla of Vater: Fifty-five consecutive resections. Arch Surg. 2003;138:941-8.

39. Heinrich S, Clavien PA. Ampullary cancer. Curr Opin Gastroenterol. $2010 ; 26: 280-5$.

40. Vauthey JN, Blumgart LH. Recent Advances in the Management of Cholangiocarcinomas. Semin Liver Dis. 1994;14(2):109-14.

41. Nakeeb A, Pitt HA, Sohn TA, Coleman JA, Abrams RA, Piantadosi S, et al. Cholangiocarcinoma: A spectrum of intrahepatic, perihilar, and distal tumors. Ann Surg. 1996;224(4):463-75.

42. Hallet J, Law CHL, Cukier M, Saskin R, Liu N, Singh S. Exploring the rising incidence of neuroendocrine tumors: A population-based analysis of epidemiology, metastatic presentation, and outcomes. Cancer. 2015;121(4):58997.

43. Dasari A, Shen C, Halperin D, Zhao B, Zhou S, Xu Y, et al. Trends in the 
incidence, prevalence, and survival outcomes in patients with neuroendocrine tumors in the United States. JAMA Oncol. 2017;3(10):1335-42.

44. Kasumova GG, Tabatabaie O, Eskander MF, Tadikonda A, Ng SC, Tseng JF. National Rise of Primary Pancreatic Carcinoid Tumors: Comparison to Functional and Nonfunctional Pancreatic Neuroendocrine Tumors. J Am Coll Surg. 2017;224(6):1057-64.

45. Doi R. Determinants of surgical resection for pancreatic neuroendocrine tumors. J Hepatobiliary Pancreat Sci. 2015;22(8):610-7.

46. Liu JB, Baker MS. Surgical Management of Pancreatic Neuroendocrine Tumors. Surg Clin North Am. 2016;96(6):1447-68.

47. Lurie RH. NCCN Clinical Practice Guidelines in Oncology [Internet]. 2015. Available $\quad$ from: $\quad$ http://www.iqanda-cme.com/assets/pdf/NCCN Guidelines_Neuroendocrine Tumors.pdf

48. Cloyd JM, Norton JA, Visser BC, Poultsides GA. Does the Extent of Resection Impact Survival for Duodenal Adenocarcinoma? Analysis of 1,611 Cases. Ann Surg Oncol. 2014;22(2):573-80.

49. Zhang S, Cui Y, Zhong B, Xiao W, Gong X, Chao K, et al. Clinicopathological characteristics and survival analysis of primary duodenal cancers: A 14-year experience in a tertiary centre in South China. Int $\mathrm{J}$ Colorectal Dis. 2011;26(2):219-26.

50. Birkmeyer JD, Sun Y, Wong SL, Stukel TA. Hospital volume and late survival after cancer surgery. Ann Surg. 2007;245(5):777-83.

51. Giuliano K, Ejaz A, He J. Technical aspects of pancreaticoduodenectomy and their outcomes. Chinese Clin Oncol. 2017;6(4):901-901.

52. Seiler CA, Wagner M, Buchler MW. The role of pylorus-preserving 
duodenopancreatectomy in pancreatic cancer. Dig Surg. 1994;11(3-6):390-6.

53. Hackert T, Probst P, Knebel P, Doerr-Harim C, Bruckner T, Klaiber U, et al. Pylorus Resection Does Not Reduce Delayed Gastric Emptying after Partial Pancreatoduodenectomy A Blinded Randomized Controlled Trial (PROPP Study, DRKS00004191). Ann Surg. 2018;267(6):1021-7.

54. Akizuki E, Kimura Y, Nobuoka T, Imamura M, Nishidate T, Mizuguchi T, et al. Prospective nonrandomized comparison between pylorus-preserving and subtotal stomach-preserving pancreaticoduodenectomy from the perspectives of DGE occurrence and postoperative digestive functions. J Gastrointest Surg. 2008;12(7):1185-92.

55. Kurahara H, Takao S, Shinchi H, Mataki Y, Maemura K, Sakoda M, et al. Subtotal stomach-preserving pancreaticoduodenectomy (SSPPD) prevents postoperative delayed gastric emptying. J Surg Oncol. 2010;102(6):615-9.

56. Matsumoto I, Shinzeki M, Asari S, Goto T, Shirakawa S, Ajiki T, et al. A prospective randomized comparison between pylorus- and subtotal stomachpreserving pancreatoduodenectomy on postoperative delayed gastric emptying occurrence and long-term nutritional status. J Surg Oncol. 2014;109(7):690-6.

57. Kawai M, Tani M, Hirono S, Miyazawa M, Shimizu A, Uchiyama K, et al. Pylorus ring resection reduces delayed gastric emptying in patients undergoing pancreatoduodenectomy: A prospective, randomized, controlled trial of pylorusresecting versus pylorus-preserving pancreatoduodenectomy. Ann Surg. 2011;253(3):495-501.

58. Hayashibe A, Kameyama M, Shinbo M, Makimoto S. The Surgical Procedure and Clinical Results of Subtotal Stomach Preserving Pancreaticoduodenectomy (SSPPD) in Comparison With Pylorus Preserving Pancreaticoduodenectomy 
(PPPD). J Surg Oncol. 2007;95:106-10.

59. Zhou Y, Lin L, Wu L, Xu D, Li B. A case-matched comparison and meta-analysis comparing pylorus-resecting pancreaticoduodenectomy with pylorus-preserving pancreaticoduodenectomy for the incidence of postoperative delayed gastric emptying. HPB. 2015;17(4):337-43.

60. Fujii T, Kanda M, Kodera Y, Nagai S, Sahin TT, Hayashi M, et al. Preservation of the pyloric ring has little value in surgery for pancreatic head cancer: A comparative study comparing three surgical procedures. Ann Surg Oncol. 2012;

61. Oida T, Mimatsu K, Kano H, Kawasaki A, Kuboi Y, Fukino N, et al. Preventing delayed gastric emptying in pancreaticogastrostomy by a modified subtotalstomach-preserving pancreaticoduodenectomy: Oida modification. Hepatogastroenterology. 2011;58:13684-8.

62. Hackert T, Hinz U, Hartwig W, Strobel O, Fritz S, Schneider L, et al. Pylorus resection in partial pancreaticoduodenectomy: Impact on delayed gastric emptying. Am J Surg. 2013;206(3):296-9.

63. Atsushi Nanashima, Takafumi Abo, Yorihisa Sumida ST, Takashi Nonaka, Hiroaki Takeshita, Shigekazu Hidaka, Terumitsu Sawai, Toru Yasutake and TN. Comparison of Results between Pylorus-preserving Pancreaticoduodenectomy and Subtotal Stomach-preserving Pancreaticoduodenectomy: Report at a Single Cancer Institute. Hepatogastroenterology. 2013;60(125):1182-8.

64. Klaiber U, Probst P, Strobel O, Michalski CW, Dörr-Harim C, Diener MK, et al. Meta-analysis of delayed gastric emptying after pylorus-preserving versus pylorus-resecting pancreatoduodenectomy. Br J Surg. 2018;105(4):339-49.

65. Hanna M, Gadde R, Tamariz L, Allen C, Meizoso J, Sleeman D, et al. Delayed Gastric Emptying After Pancreaticoduodenectomy: Is Subtotal Stomach 
Preserving Better or Pylorus Preserving? J Gastrointest Surg. 2015;19(8):154252.

66. Chen Q jun, He Z qiang, Yang Y, Zhang Y shun, Chen X lin, Yang H ji, et al. Is there comparable morbidity in pylorus-preserving and pylorus-resecting pancreaticoduodenectomy? A meta-analysis. J Huazhong Univ Sci Technol - Med Sci. 2015;35(6):793-800.

67. Wu W, Hong X, Fu L, Liu S, You L, Zhou L, et al. The effect of pylorus removal on delayed gastric emptying after pancreaticoduodenectomy: A meta-analysis of 2,599 patients. PLoS One. 2014;9:eI08380.

68. Huang W, Xiong JJ, Wan MH, Szatmary P, Bharucha S, Gomatos I, et al. Metaanalysis of subtotal stomach-preserving pancreaticoduodenectomy vs pylorus preserving pancreaticoduodenectomy. World J Gastroenterol. 2015;21:6361-73.

69. Pallisera A, Morales R, Ramia JM. Tricks and tips in pancreatoduodenectomy. World J Gastrointest Oncol. 2014;6(9):344.

70. Sanjay P, Takaori K, Govil S, Shrikhande S V., Windsor JA. "Artery-first" approaches to pancreatoduodenectomy. Br J Surg. 2012;99(8):1027-35.

71. Pessaux P, Varma D, Arnaud JP. Pancreaticoduodenectomy: Superior Mesenteric Artery First Approach. J Gastrointest Surg. 2006;10(4):607-11.

72. Hirota M, Hashimoto D, Ishiko T, Satoh N, Takamori H, Chikamoto A, et al. Distal pancreatectomy using a no-touch isolation technique. Scand J Surg. 2012;101(3):156-9.

73. Pessaux P, Rosso E, Panaro F, Marzano E, Oussoultzoglou E, Bachellier P, et al. Preliminary experience with the hanging maneuver for pancreaticoduodenectomy. Eur J Surg Oncol. 2009;35(9):1006-10.

74. Kurosaki I, Minagawa M, Takano K, Takizawa K, Hatakeyama K. Left posterior 
approach to the superior mesenteric vascular pedicle in pancreaticoduodenectomy for cancer of the pancreatic head. J Pancreas. 2011;12(3):220-9.

75. Ironside N, Barreto SG, Loveday B, Shrikhande S V, Windsor JA. Meta-analysis of an artery-first approach versus standard pancreatoduodenectomy on perioperative outcomes and. Br J Surg. 2018;105(6):628-36.

76. Tol JAMG, Gouma DJ, Bassi C, Dervenis C. Consensus Definition of a standard lymphadenectomy in surgery for pancreatic ductal adenocarcinoma : A consensus statement by the International Study Group on Pancreatic Surgery ( ISGPS ). Surgery. 2014;156(3):591.

77. Jang J, Kang MJ, Heo JS, Choi DW, Yu HC, Kim SG, et al. A Prospective Randomized Controlled Study Comparing Outcomes of Standard Resection and Extended Resection, Including Dissection of the Nerve Plexus and Various Lymph Nodes, in Patients With Pancreatic Head Cancer. Ann Surg. 2014;259(4):656-64.

78. Yeo C, Cameron J, Lillemoe K, Sohn T, Campbell K, Sauter P, et al. Pancreaticoduodenectomy with or without distal gastrectomy and extended retroperitoneal lymphadenectomy for periampullary adenocarcinoma, part 2: randomized controlled trial evaluating survival, morbidity, and mortality. Ann Surg. 2002;236(3):355-68.

79. Japan Pancreas Society. Classification of pancreatic carcinoma. 4th ed. Kenearah \& Co. Ltd, editor. Tokyo; 2017.

80. Clerveus M, Morandeira-Rivas A, Picazo-Yeste J, Moreno-Sanz C. Pancreaticogastrostomy versus pancreaticojejunostomy after pancreaticoduodenectomy: a systematic review and meta-analysis of randomized controlled trials. J Gastrointest Surg. 2014;18(9):1693-704. 
81. Lyu Y, Li T, Cheng Y, Wang B, Chen L, Zhao S. Pancreaticojejunostomy Versus Pancreaticogastrostomy after Pancreaticoduodenectomy: An Up-to-date Metaanalysis of RCTs Applying the ISGPS (2016) Criteria. Surg Laparosc Endosc Percutaneous Tech. 2018;28(3):139-46.

82. Cheng $\mathrm{Y}$, Briarava $\mathrm{M}$, Lai $\mathrm{M}$, Wang $\mathrm{X}$, Tu $\mathrm{B}$, Cheng $\mathrm{N}$, et al. Pancreaticojejunostomy versus pancreaticogastrostomy reconstruction for the prevention of postoperative pancreatic fistula following pancreaticoduodenectomy. Cochrane Database Syst Rev. 2017;2017(9).

83. Shrikhande S V., Sivasanker M, Vollmer CM, Friess H, Besselink MG, Fingerhut A, et al. Pancreatic anastomosis after pancreatoduodenectomy: A position statement by the International Study Group of Pancreatic Surgery (ISGPS). Vol. 161, Surgery. 2017.

84. Nitsche U, Müller TC, Späth C, Cresswell L, Wilhelm D, Friess H, et al. The evidence based dilemma of intraperitoneal drainage for pancreatic resection - A systematic review and meta-analysis. BMC Surg. 2014;14(1):1-12.

85. Van Buren G, Bloomston M, Schmidt CR, Behrman SW, Zyromski NJ, Ball CG, et al. A Prospective Randomized Multicenter Trial of Distal Pancreatectomy with and Without Routine Intraperitoneal Drainage. Ann Surg. 2017;266(3):421-31.

86. Dou CW, Liu ZK, Jia YL, Zheng X, Tu KS, Yao YM, et al. Systematic review and meta-analysis of prophylactic abdominal drainage after pancreatic resection. World J Gastroenterol. 2015;21(18):5719-34.

87. Wang YC, Szatmary P, Zhu JQ, Xiong JJ, Huang W, Gomatos I, et al. Prophylactic intra-peritoneal drain placement following pancreaticoduodenectomy: A systematic review and metaanalysis. World J Gastroenterol. 2015;21(8):2510-21.

88. Witzigmann H, Diener MK, Kißenkötter S, Rossion I, Bruckner T, Werner B, et 
al. No need for routine drainage after pancreatic head resection: The dual-center, randomized, controlled PANDRA trial (ISRCTN04937707). Ann Surg. 2016;264(3):528-35.

89. Goh BKP, Tan YM, Chung YFA, Cheow PC, Ong HS, Chan WH, et al. Critical appraisal of 232 consecutive distal pancreatectomies with emphasis on risk factors, outcome, and management of the postoperative pancreatic fistula: A 21-year experience at a single institution. Arch Surg. 2008;143(10):956-65.

90. Strasberg SM, Fields R. Left-Sided Pancreatic Cancer Distal Pancreatectomy and its Variants: Radical Antegrade Modular Pancreatosplenectomy and Distal Pancreatectomy With Celiac Axis Resection. cancer J. 2012;18(6):562-70.

91. Klompmaker S, van Hilst J, Gerritsen SL, Adham M, Teresa Albiol Quer M, Bassi C, et al. Outcomes After Distal Pancreatectomy with Celiac Axis Resection for Pancreatic Cancer: A Pan-European Retrospective Cohort Study. Ann Surg Oncol. 2018;25(5):1440-7.

92. Riviere D, Gurusamy KS, Kooby DA, Vollmer CM, Besselink MGH, Davidson BR, et al. Laparoscopic versus open distal pancreatectomy for pancreatic cancer. Cochrane Database Syst Rev. 2016;(4).

93. Gavriilidis P, Lim C, Menahem B, Lahat E, Salloum C, Azoulay D. Robotic versus laparoscopic distal pancreatectomy - The first meta-analysis. Hpb. 2016;18(7):567-74.

94. Guerrini GP, Lauretta A, Belluco C, Olivieri M, Forlin M, Basso S, et al. Robotic versus laparoscopic distal pancreatectomy: An up-to-date meta-analysis. BMC Surg. 2017;17(1):1-10.

95. Mallet-Guy P, Vachon A. Pancreatites chroniques gauches. Paris Masson; 1943.

96. Warshaw AL. Distal pancreatectomy with preservation of the spleen. Arch Surg. 
1988;123(5):550-3.

97. He Z, Qian D, Hua J, Gong J, Lin S, Song Z. Clinical Comparison of Distal Pancreatectomy with or without Splenectomy: A Meta-Analysis. PLoS One. 2014;9(3):e91593.

98. Fang WL, Su CH, Shyr YM, Chen TH, Lee RC, Tai LC, et al. Functional and morphological changes in pancreatic remnant after pancreaticoduodenectomy. Pancreas. 2007;35(4):361-5.

99. Briggs CD, Mann CD, Irving GRB, Neal CP, Peterson M, Cameron IC, et al. Systematic review of minimally invasive pancreatic resection. J Gastrointest Surg. 2009;13(6):1129-37.

100. Zhou Y, Zhao M, Wu L, Ye F, Si X. Short- and long-term outcomes after enucleation of pancreatic tumors: An evidence-based assessment. Pancreatology. 2016;16(6):1092-8.

101. Goldstein MJ, Toman J, Chabot JA. Pancreaticogastrostomy: A Novel Application after Central Pancreatectomy. 2004;198(6):871-6.

102. Goudard Y, Gaujoux S, Dokmak S, Cros J, Couvelard A, Palazzo M, et al. Reappraisal of central pancreatectomy a 12-year single-center experience. JAMA Surg. 2014;149(4):356-63.

103. Iacono C, Verlato G, Ruzzenente A, Campagnaro T, Bacchelli C, Valdegamberi A. Systematic review of central pancreatectomy and meta-analysis of central versus distal pancreatectomy. 2013;100(7):873-85.

104. Nathan H, Wolfgang CL, Edil BH, Choti MA, Herman JM, Schulick RD, et al. Peri-Operative Mortality and Long-Term Survival after Total Pancreatectomy for Pancreatic Adenocarcinoma: A Population-Based Perspective. J Surg Oncol. 2009;99(2):87-92. 
105. Andrén-sandberg Å, Ansorge C, Yadav D. Are There Indications for Total Pancreatectomy in 2016? Dig Surg. 2016;33(4):329-34.

106. Almond M, Roberts KJ, Hodson J, Sutcliffe R, Marudanayagam R, Isaac J, et al. Changing indications for a total pancreatectomy : perspectives over a quarter of a century. 2015;416-21.

107. Alexakis N, Ghaneh P, Connor S, Raraty M, Sutton R, Neoptolemos JP. Duodenum- and spleen-preserving total pancreatectomy for end-stage chronic pancreatitis. Br J Surg. 2003;90(11):1401-8.

108. Giulianotti PC, Addeo P, Buchs N, Bianco F, Ayloo S. Early experience with robotic total pancreatectomy. Pancreas. 2015;40(2):311-3.

109. Simons JP, Shah SA, Ng SC, Whalen GF, Tseng JF. National Complication Rates after Pancreatectomy: Beyond Mere Mortality. J Gastrointest Surg. 2009;13(10):1798-805.

110. Winter JM, Cameron JL, Campbell KA, Hruban RH, Riall TS, Schulick RD, et al. 1423 Pancreaticoduodenectomies for Pancreatic Cancer: A Single-Institution Experience. J Gastrointest Surg. 2006;10(9):1199-211.

111. Vin Y, Sima CS, Getrajdman GI, Brown KT, Covey A, Brennan MF, et al. Management and Outcomes of Postpancreatectomy Fistula, Leak, and Abscess : Results of 908 Patients Resected at a Single Institution Between 2000 and 2005. J Am Coll Surg. 2008;207(4):490-8.

112. Okano K, Hirao T, Unno M, Fujii T, Yoshitomi H, Suzuki S, et al. Postoperative infectious complications after pancreatic resection. 2015;1551-60.

113. Teh SH, Diggs BS, Deveney CW, Sheppard BC. Patient and Hospital Characteristics on the Variance of Perioperative Outcomes for Pancreatic Resection in the United States. Arch Surg. 2009;144(8):713-21. 
114. Dindo D, Demartines N, Clavien P. Classification of Surgical Complications: a new proposal with evaluation in a cohort of 6336 patients and results of a survey. Ann Surg. 2004;240(2):205-13.

115. Bassi C, Marchegiani G, Dervenis C, Sarr M, Abu Hilal M, Adham M, et al. The 2016 update of the International Study Group (ISGPS) definition and grading of postoperative pancreatic fistula: 11 Years After. Surgery. 2017;161(3):584-91.

116. Zhang H, Zhu F, Shen M, Tian R, Shi CJ, Wang X, et al. Systematic review and meta-analysis comparing three techniques for pancreatic remnant closure following distal pancreatectomy. Br J Surg. 2015;102(1):4-15.

117. Barreto G, D’Souza MA, Shukla PJ, Shrikhande S V. The gray zone between postpancreaticoduodenectomy collections and pancreatic fistula. Pancreas. 2008;37(4):422-5.

118. Yang Y, Tian X, Zhuang Y, Wang W, Wan Y, Huang Y. Risk factors of pancreatic leakage after pancreaticoduodenectomy. World J Gastroenterol. 2005;11(16):2456-61.

119. Choe YM, Lee KY, Oh CA, Lee JB, Choi SK, Hur YS, et al. Risk factors affecting pancreatic fistulas after pancreaticoduodenectomy. World J Gastroenterol. 2008;14(45):6970-4.

120. Gans SL, Van Westreenen HL, Kiewiet JJS, Rauws EAJ, Gouma DJ, Boermeester MA. Systematic review and meta-analysis of somatostatin analogues for the treatment of pancreatic fistula. Br J Surg. 2012;99(66):754-60.

121. Wente MN, Bassi C, Dervenis C, Fingerhut A, Gouma DJ, Izbicki JR, et al. Delayed gastric emptying (DGE) after pancreatic surgery: A suggested definition by the International Study Group of Pancreatic Surgery (ISGPS). Surgery. 2007;142(5):761-8. 
122. Martignoni ME, Friess H, Sell F, Ricken L, Shrikhande S, Kulli C, et al. Enteral nutrition prolongs delayed gastric emptying in patients after whipple resection. Am J Surg. 2000;180(1):18-23.

123. Richter A, Niedergethmann M, Sturm JW, Lorenz D, Post S, Trede M. Long-term results of partial pancreaticoduodenectomy for ductal adenocarcinoma of the pancreatic head: 25-Year experience. World J Surg. 2003;27(3):324-9.

124. Nimptsch U, Krautz C, Weber GF, Mansky T, Grutzmann R. Nationwide inhospital mortality following pancreatic surgery in Germany is higher than anticipated. Ann Surg. 2016;264(6):1082-90.

125. De Castro SMM, Kuhlmann KFD, Busch ORC, Van Delden OM, Laméris JS, Van Gulik TM, et al. Delayed massive hemorrhage after pancreatic and biliary surgery: Embolization or surgery? Ann Surg. 2005;241(1):85-91.

126. Tien YW, Lee PH, Yang CY, Ho MC, Chiu YF. Risk factors of massive bleeding related to pancreatic leak after pancreaticoduodenectomy. J Am Coll Surg. 2005;201(4):554-9.

127. de Castro SM, Busch OR, Gouma DJ. Management of bleeding and leakage after pancreatic surgery. Best Pr Res Clin Gastroenterol. 2004;18(5):847-64.

128. Wente MN, Veit JA, Bassi C, Dervenis C, Fingerhut A, Gouma DJ, et al. Postpancreatectomy hemorrhage (PPH)-An International Study Group of Pancreatic Surgery (ISGPS) definition. Surgery. 2007;142(1):20-5.

129. Aoki S, Miyata H, Konno H, Gotoh M, Motoi F, Kumamaru H, et al. Risk factors of serious postoperative complications after pancreaticoduodenectomy and risk calculators for predicting postoperative complications: a nationwide study of 17,564 patients in Japan. J Hepatobiliary Pancreat Sci. 2017;24(5):243-51.

130. Braga M, Capretti G, Pecorelli N, Balzano G, Doglioni C, Ariotti R, et al. A 
prognostic score to predict major complications after pancreaticoduodenectomy. Ann Surg. 2011;254(5):702-7.

131. Callery MP, Pratt WB, Kent TS, Chaikof EL, Vollmer CM. A prospectively validated clinical risk score accurately predicts pancreatic fistula after pancreatoduodenectomy. J Am Coll Surg. 2013;216(1):1-14.

132. Gleeson EM, Shaikh MF, Shewokis PA, Clarke JR, Meyers WC, Pitt HA, et al. WHipple-ABACUS, a simple, validated risk score for 30-day mortality after pancreaticoduodenectomy developed using the ACS-NSQIP database. Surgery. 2016;160(5):1279-87.

133. Greenblatt DY, Kelly KJ, Rajamanickam V, Wan Y, Hanson T, Rettammel R, et al. Preoperative factors predict perioperative morbidity and mortality after pancreaticoduodenectomy. Ann Surg Oncol. 2011;18(8):2126-35.

134. Kantor O, Talamonti MS, Pitt HA, Vollmer CM, Riall TS, Hall BL, et al. Using the NSQIP Pancreatic Demonstration Project to Derive a Modified Fistula Risk Score for Preoperative Risk Stratification in Patients Undergoing Pancreaticoduodenectomy. J Am Coll Surg. 2017;224(5):816-25.

135. Venkat R, Cameron JL, Makary MA, Edil BH. Predicting the Risk of Perioperative Mortality in Patients Undergoing Pancreaticoduodenectomy. Arch Surg. 2011;146(11):1277.

136. Uzunoglu FG, Reeh M, Vettorazzi E, Ruschke T, Hannah P, Nentwich MF, et al. Preoperative pancreatic resection (PREPARE) Score: A prospective multicenterbased morbidity risk score. Ann Surg. 2014;260(5):857-64.

137. Wiltberger G, Muhl B, Benzing C, Atanasov G, Hau HM, Horn M, et al. Preoperative risk stratification for major complications following pancreaticoduodenectomy: Identification of high-risk patients. Int J Surg. 
2016;31:33-9.

138. Rodriguez-Lopez M, Tejero-Pintor FJ, Perez-Saborido B, Barrera-Rebollo A, Bailon-Cuadrado M, Pacheco-Sanchez D. Severe morbidity after pancreatectomy is accurately predicted by preoperative pancreatic resection score (PREPARE): A prospective validation analysis from a medium-volume center. Hepatobiliary Pancreat Dis Int. 2018;17(6):559-65.

139. Celik H, Kilic MO, Erdogan A, Ceylan C, Tez M. External validation of PREPARE score in Turkish patients who underwent pancreatic surgery: To the Editor. Hepatobiliary Pancreat Dis Int. 2016;15(1):108-9.

140. Rodriguez-Lopez M, Bailon-Cuadrado M, Tejero-Pintor FJ, Perez-Saborido B, Asensio-Diaz E, Barrera-Rebollo A. Preoperative pancreatic resection score: a preliminary prospective validation from Spain. Hepatobiliary Pancreat Dis Int. 2016;15(6):667-8.

141. Samim M, Mungroop TH, AbuHilal M, Isfordink CJ, Molenaar QI, van der Poel MJ, et al. Surgeons' assessment versus risk models for predicting complications of hepato-pancreato-biliary surgery (HPB-RISC): a multicenter prospective cohort study. HPB. 2018;20(9):809-14.

142. Rooij T De, Tol JA, Eijck CH Van, Boerma D. Outcomes of Distal Pancreatectomy for Pancreatic Ductal Adenocarcinoma in the Netherlands: A Nationwide Retrospective Analysis. 2016;23(2):585-91.

143. Čečka F, Jon B, Čermáková E, Šubrt Z, Alexander F. Impact of postoperative complications on clinical and economic consequences in pancreatic surgery. Ann Surg Treat Res. 2016;90(1):21-8.

144. Parikh P, Shiloach M, Cohen ME, Bilimoria KY, Ko CY, Hall BL, et al. Pancreatectomy risk calculator: An ACS-NSQIP resource. HPB. 2010;12(7):488- 
97.

145. Murad MH, Alsawas M, Asi N, Alahdab F. The new evidence pyramid. Evid Based Med. 2016;21(4):125-7.

146. Insulander J, Sanjeevi S, Haghighi M, Ivanics T, Analatos A, Lundell L, et al. Prognosis following surgical bypass compared with laparotomy alone in unresectable pancreatic adenocarcinoma. Br J Surg. 2016;103(9):1200-8.

147. Scheufele F, Schorn S, Demir IE, Sargut M, Tieftrunk E, Calavrezos L, et al. Preoperative biliary stenting versus operation first in jaundiced patients due to malignant lesions in the pancreatic head: A meta-analysis of current literature. Surgery. 2017;161(4):939-50.

148. Collard M, Marchese T, Guedj N, Cauchy F, Chassaing C, Ronot M, et al. Is Routine Splenectomy Justified for All Left-Sided Pancreatic Cancers? Histological Reappraisal of Splenic Hilar Lymphadenectomy. Ann Surg Oncol. 2019;26(4):1071-8.

149. Pendola F, Gadde R, Ripat C, Sharma R, Picado O, Lobo L, et al. Distal pancreatectomy for benign and low grade malignant tumors: Short-term postoperative outcomes of spleen preservation-A systematic review and update meta-analysis. J Surg Oncol. 2017;115(2):137-43.

150. Montiel Casado MC, Pardo Sánchez F, Rotellar Sastre F, Martí Cruchaga P, Álvarez Cienfuegos FJ. Experiencia de un programa de fast-track en la duodenopancreatectomía cefálica. Cir Esp. 2010;87(6):378-84.

151. Ramia JM, Serrablo A, Gomez Bravo MA. National survey on Pancreatic Surgery Units. Cir Esp. 2019;97(5):254-60.

152. Cameron JL, He J. Two thousand consecutive pancreaticoduodenectomies. J Am Coll Surg. 2015;220(4):530-6. 
153. Pecorelli N, Balzano G, Capretti G, Zerbi A, Di Carlo V, Braga M. Effect of Surgeon Volume on Outcome Following Pancreaticoduodenectomy in a HighVolume Hospital. J Gastrointest Surg. 2012;16(3):518-23.

154. Krautz C, Nimptsch U, Weber GF, Mansky T, Grützmann R. Effect of Hospital Volume on In-hospital Morbidity and Mortality Following Pancreatic Surgery in Germany. Ann Surg. 2018;267(3):411-7.

155. Goodney PP, Stukel TA, Lucas FL, Finlayson EVA, Birkmeyer JD. Hospital Volume, Length of Stay, and Readmission Rates in High-Risk Surgery. Ann Surg. 2003;238(2):161-7.

156. Sánchez Cabús S, Saavedra D, Sampson J, Cubel M, López-Boado MÁ, Ferrer J, et al. Resultados de la pancreatogastroanastomosis con bipartición gástrica después de duodenopancreatectomía con preservación pilórica. Cir Española. 2015;93(8):502-8.

157. Dominguez-Comesaña E, Gonzalez-Rodriguez FJ, Ulla-Rocha JL, LedeFernandez Á, Portela-Serra JL, Piñon-Cimadevila MÁ. Morbimortalidad de la resección pancreática. Cir Española. 2013;91(10):651-8.

158. Pedrazzoli S. Pancreatoduodenectomy (PD) and postoperative pancreatic fistula (POPF). Medicine (Baltimore). 2017;96(19).

159. Morales Soriano R, Esteve Pérez N, Tejada Gavela S, Cuadrado García Á, Rodríguez Pino JC, Morón Canis JM, et al. Resultados de la rehabilitación multimodal en la duodenopancreatectomía cefálica. Cir Española. 2015;93(8):509-15.

160. Landi F, Dopazo C, Sapisochin G, Beisani M, Blanco L, Caralt M, et al. Resultados a largo plazo de la duodenopancreatectomía cefálica con resección de la vena mesentérica superior y vena porta por adenocarcinoma de la cabeza de páncreas. 
Cir Española. 2015;93(8):522-9.

161. Herrera-Cabezón FJ, Sánchez-Acedo P, Zazpe-Ripa C, Tarifa-Castilla A, LeraTricas JM. Estándares de calidad en 480 resecciones pancreáticas: estudio prospectivo observacional. Rev Española Enfermedades Dig. 2015;107(3):14351.

162. Figueras J, Sabater L, Planellas P, Muñoz-Forner E, Lopez-Ben S, Falgueras L, et al. Randomized clinical trial of pancreaticogastrostomy versus pancreaticojejunostomy on the rate and severity of pancreatic fistula after pancreaticoduodenectomy. Br J Surg. 2013;100(12):1597-605.

163. Beane JD, House MG, Miller A, Nakeeb A, Schmidt CM, Zyromski NJ, et al. Optimal management of delayed gastric emptying after pancreatectomy: An analysis of 1,089 patients. Surgery. 2014;156(4):939-48.

164. Park YC, Kim SW, Jang JY, Ahn YJ, Park YH. Factors influencing delayed gastric emptying after pylorus-preserving pancreatoduodenectomy. J Am Coll Surg. 2003;196(6):859-65.

165. Duarte Garcés AA, Andrianello S, Marchegiani G, Piccolo R, Secchettin E, Paiella S, et al. Reappraisal of post-pancreatectomy hemorrhage (PPH) classifications: do we need to redefine grades A and B? HPB. 2018;20(8):702-7.

166. Grützmann R, Rückert F, Hippe-Davies N, Distler M, Saeger HD. Evaluation of the International Study Group of Pancreatic Surgery definition of postpancreatectomy hemorrhage in a high-volume center. Surgery. 2012;151(4):61220.

167. Angiolini MR, Gavazzi F, Ridolfi C, Moro M, Morelli P, Montorsi M, et al. Role of C-Reactive Protein Assessment as Early Predictor of Surgical Site Infections Development after Pancreaticoduodenectomy. Dig Surg. 2016;33(4):267-75. 
168. Yeo CJ, Cameron JL, Sohn TA, Lillemoe KD, Pitt HA, Talamini MA, et al. Six Hundred Fifty Consecutive Pancreaticoduodenectomies in the 1990s. Ann Surg. 1997;226(3):248-60.

169. Rubio-Manzanares-Dorado M, Marín-Gómez LM, Aparicio-Sánchez D, SuárezArtacho G, Bellido C, Álamo JM, et al. Implicaciones de las variantes arteriales hepáticas durante la duodenopancreatectomía cefálica oncológica. Rev Española Enfermedades Dig. 2015;107(7):417-22.

170. Sabater L, Mora I, Gámez del Castillo JM, Escrig-Sos J, Muñoz-Forner E, GarcésAlbir M, et al. Outcome quality standards in pancreatic oncologic surgery in Spain. Cir Española. 2018;96(6):342-51.

171. Are C, Afuh C, Ravipati L, Sasson A, Ullrich F, Smith L. Preoperative nomogram to predict risk of perioperative mortality following pancreatic resections for malignancy. J Gastrointest Surg. 2009;13(12):2152-62.

172. Velez-Serrano JF, Velez-Serrano D, Hernandez-Barrera V, Jimenez-Garcia R, De Andres AL, Garrido PC, et al. Prediction of in-hospital mortality after pancreatic resection in pancreatic cancer patients: A boosting approach via a populationbased study using health administrative data. PLoS One. 2017;12(6):1-13.

173. Klompmaker S, van Hilst J, Wellner UF, Busch OR, Coratti A, D’Hondt M, et al. Outcomes After Minimally-invasive Versus Open Pancreatoduodenectomy. Ann Surg. 2018;Doi: 10.1097/sla.0000000000002850 [Ahead of print].

174. Schorn S, Demir IE, Vogel T, Schirren R, Reim D, Wilhelm D, et al. Mortality and postoperative complications after different types of surgical reconstruction following pancreaticoduodenectomy — a systematic review with meta-analysis. Langenbeck's Arch Surg. 2019;404(2):141-57.

175. Xiong J, Szatmary P, Huang W, Iglesia-Garcia DD La, Nunes QM, Xia Q, et al. 
Enhanced recovery after surgery program in patients undergoing pancreaticoduodenectomy a prisma-compliant systematic review and metaanalysis. Medicine (Baltimore). 2016;95(18):e3497.

176. Xu X, Zheng C, Zhao Y, Chen W, Huang Y. Enhanced recovery after surgery for pancreaticoduodenectomy: Review of current evidence and trends. Int J Surg. 2018;50:79-86.

177. Kagedan DJ, Ahmed M, Devitt KS, Wei AC. Enhanced recovery after pancreatic surgery: A systematic review of the evidence. HPB. 2015;17(1):11-6.

178. Collins G, Reitsma J, Altman D, Moons K. Erratum: Transparent reporting of a multivariable prediction model for individual prognosis or diagnosis (TRIPOD): The TRIPOD statement (Annals of Internal Medicine). $\mathrm{Br} \mathrm{J}$ Surg. 2015;102(3):148-58. 
Esta Tesis comenzó a escribirse en Nueva York, cerca del Hospital Presbyterian, donde un cirujano llamado Allen O. Whipple sistematizó por primera vez la técnica quirúrgica conocida por su apellido. 